\title{
WestVirginiaUniversity
}

THE RESEARCH REPOSITORY @ WVU

Graduate Theses, Dissertations, and Problem Reports

2008

\section{Measurement of short-wavelength electrostatic fluctuations in a helicon plasma source}

\author{
Robert Aaron Hardin \\ West Virginia University
}

Follow this and additional works at: https://researchrepository.wvu.edu/etd

\section{Recommended Citation}

Hardin, Robert Aaron, "Measurement of short-wavelength electrostatic fluctuations in a helicon plasma source" (2008). Graduate Theses, Dissertations, and Problem Reports. 2723.

https://researchrepository.wvu.edu/etd/2723

This Dissertation is protected by copyright and/or related rights. It has been brought to you by the The Research Repository @ WVU with permission from the rights-holder(s). You are free to use this Dissertation in any way that is permitted by the copyright and related rights legislation that applies to your use. For other uses you must obtain permission from the rights-holder(s) directly, unless additional rights are indicated by a Creative Commons license in the record and/ or on the work itself. This Dissertation has been accepted for inclusion in WVU Graduate Theses, Dissertations, and Problem Reports collection by an authorized administrator of The Research Repository @ WVU.

For more information, please contact researchrepository@mail.wvu.edu. 


\title{
Measurement of Short-Wavelength Electrostatic Fluctuations in a Helicon Plasma Source
}

\author{
Robert Aaron Hardin \\ Dissertation submitted to the College of Arts and Sciences at \\ West Virginia University \\ in partial fulfillment of the requirements for the degree of \\ Doctor of Philosophy \\ in \\ Plasma Physics \\ Earl E. Scime, PhD., Chair \\ Mark. E. Koepke, PhD. \\ John E. Littleton, PhD. \\ John L. Kline, PhD. \\ Brian Woerner, PhD. \\ Department of Physics \\ Morgantown, West Virginia \\ 2008
}

Keywords: Helicon plasma source, Trivelpiece-Gould, Parametric instability, Lower hybrid resonance, Collective Thomson scattering, Quasioptical propagation, Homodyne detection 


\section{Abstract \\ Measurement of Short-Wavelength Electrostatic Fluctuations in a Helicon Plasma Source \\ Robert Aaron Hardin}

The principle objective of this work is to determine if short wavelength fluctuations capable of heating ions are excited in helicon sources at the same plasma parameters for which anomalous ion heating has been observed in helicon sources. A portable $300 \mathrm{GHz}$ based, coherent Thomson scattering (CTS) diagnostic, employing both quasioptical propagation and a homodyne detection scheme, was designed and installed on the HELIX source to measure fluctuations with wavelengths on the order of $1 \mathrm{~mm}$. While testing a new antenna designed to directly excite finite $k_{\perp}$ electrostatic waves in conjunction with a new electrostatic double probe, spontaneously occurring excited waves with wave numbers measureable with the scattering diagnostic were found. For plasma conditions shown to produce the largest amplitude, radially localized fluctuations, as measured with an electrostatic double probe, the CTS diagnostic observed a statistically significant scattered wave power at a frequency of $f \sim 100 \mathrm{kHz}$ and a perpendicular wave number of $k_{\perp} \sim 89 \mathrm{rad} / \mathrm{cm}$. While the wave frequency found with the CTS diagnostic is lower than expected for the fluctuations given the electrostatic probe measurements, the phase velocity of the waves is small enough that the waves can interact with the bulk of the ion distribution. 


\section{Acknowledgements}

First of all I would like to thank my parents; Bob and Cathy Hardin (see I can use your names and "parents" together). I am forever indebted to you both for providing me every opportunity imaginable, considering I always wanted to be involved in everything under the sun. Words could never express the love and admiration that I have for the both of you. I dedicate this work to you, for all that you have done to offer me the opportunity to follow my dreams... and so that all that training wouldn't go down the tubes. To my wonderful wife Amanda, while there may be times we may be apart, always know that you are always in my heart and soul. Oh, and by the way, you're welcome for the easy to spell last name.

Many thanks go to my advisor Earl Scime. I am very grateful for the opportunity to learn and grow as a scientist under your tutelage. Your kind patience is greatly appreciated especially the few times I may have needed a good nudge to keep things progressing. Thanks to my committee for taking time from your busy schedules to review this work. I am grateful to Mark Koepke for the helpful scientific discussions over the years. I appreciate the time and effort John Kline spent setting up my opportunity to travel to Los Alamos National Laboratory.

To the shop guys Carl, Doug, Tom, and Phil: your time, experience, and patience, especially when I needed something made last week, made my experience here quite enjoyable. Sherry and Siobhan all your help is greatly appreciated, for all the times I needed something. Many thanks go to John Heard for your effort in getting the original design of the scattering diagnostic setup, making this work possible. Costel Biloiu, Alex Hansen and Amy Keesee, your helpful discussions and insights are greatly appreciated. To the undergrads: Ryan Murphy, Zane Harvey, Steve Przybysz, and Justin Ellis, it has been a pleasure to work with fine individuals such as yourselves.

To Sean Finnegan, it has been a pleasure to have made such a good friend here at WVU. Between all the studying, video games, and countless other activities over the years, your friendship and support both personally and professionally has really made the journey here memorable. 


\section{Table of Contents}

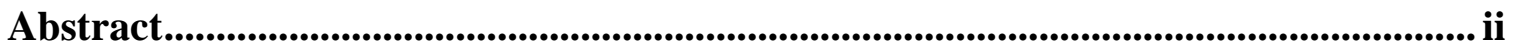

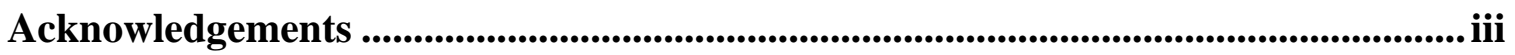

Table of Contents ................................................................................................... iv

Chapter 1: Introduction ................................................................................. 1

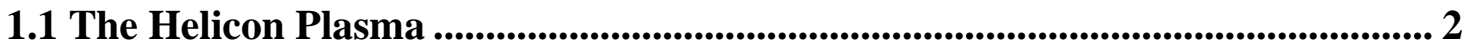

1.2 The Lower Hybrid Wave Resonance and the Slow Wave................................. 7

1.3 Fluctuation Measurement by Collective Thomson Scattering........................... 13

Chapter 1 References................................................................................. 21

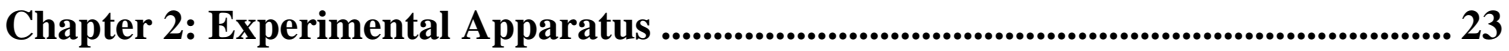

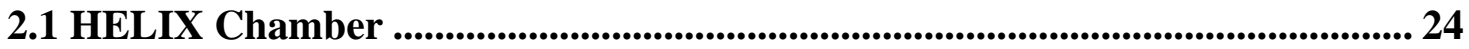

2.2 Vacuum System ................................................................................................... 27

2.3 Magnetic Field Generation................................................................................ 30

2.4 RF Antenna and Matching Network.......................................................... 32

2.5 Electrostatic Wave Launching Antenna .................................................. 33

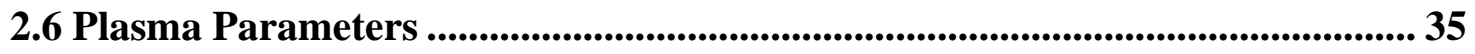

Chapter 2 References........................................................................................................... 37

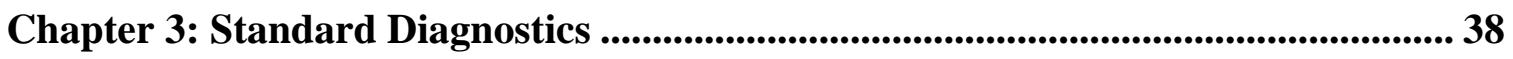

3.1 Measurement of Plasma Density and Electron Temperature........................... 38

3.1.1 Langmuir Probe Theory............................................................................................. 38

3.1.2 Langmuir Probe Apparatus............................................................................... 43

3.2 Electrostatic Fluctuation Measurement..................................................... 45

3.2.1 Electrostatic Probe................................................................................... 45

3.2.2 Electrostatic Probe Analysis ............................................................................. 47

3.3 Laser Induced Fluorescence (LIF) .............................................................. 57

3.3.1 LIF Theory ….......................................................................................................... 58

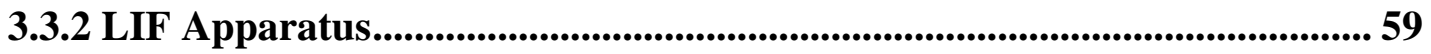

3.3.3 LIF Data Analysis .............................................................................................. 61

Chapter 3 References..........................................................................................62 62

Chapter 4: Cold Plasma Theory of the Helicon Plasma Source............................... 63 
4.1 The Cold Plasma Dispersion Relation.......................................................................... 64

4.2 Model Predictions for the CTS Diagnostic ..................................................... 74

Chapter 4 References........................................................................................ 80

Chapter 5: Collective Thomson Scattering........................................................... 81

Chapter 5 References....................................................................................................... 92

Chapter 6: 300 GHz Scattering Diagnostic Development .................................... 93

6.1 Quasioptical Gaussian Beam Propagation Theory ........................................... 94

6.2 300 GHz Diagnostic Design ............................................................................. 107

6.2.1 $300 \mathrm{GHz}$ Source and Detector......................................................................... 107

6.2.2 Optical Design .................................................................................... 109

6.3 Installation and Testing of the Scattering System and Components ............. 115

6.3.1 HDPE Lenses and Windows ....................................................................... 116

6.3.2 Beam Splitters ..................................................................................... 118

6.3.3 Vacuum Collection Mirror Apparatus .................................................... 120

6.3.4 RF Shielding ....................................................................................... 124

6.4 Proof-of-Concept Test of the 300 GHz diagnostic ........................................... 125

Chapter 6 References........................................................................................... 133

Chapter 7: Electrostatic Wave Measurements.......................................................... 134

7.1 Experimental Conditions........................................................................ 134

7.2 Collective Thomson Scattering Measurements - Part 1 .................................... 140

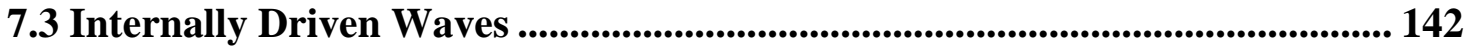

7.4 Characteristics of the Spontaneously Excited Fluctuations.......................... 148

7.5 Collective Thomson Scattering Measurements - Part 2 ................................ 156

7.6 Electrostatic Wave Investigations ............................................................................ 163

Chapter 7 References......................................................................................... 171

Chapter 8: Discussion .................................................................................................... 172

Chapter 8 References.................................................................................................. 176

Chapter 9: Conclusions .............................................................................................................. 177

Appendix A: Pressure Calibration Data..................................................................... 179

Appendix B: Cold Plasma Dispersion Relation Modeling Code ................................. 183 
Appendix C: Alignment and General Calibration Guidelines for the $300 \mathrm{GHz}$ Scattering Diagnostic ........................................................................................................ 189

Appendix D: Spectral Density Calculation Code.......................................................... 195

Vitae ...................................................................................................................................... 203 


\section{Chapter 1: Introduction}

"Roads? Where we're going... we don't need roads"

-- Dr. Emmit Brown

The helicon plasma source, as it is known today, was originally developed by Rod Boswell while at Flinders University of South Australia. ${ }^{1,2,4}$ Those first experiments produced plasmas with densities on the order of $10^{13} \mathrm{~cm}^{-3}$ with the characteristic argon "blue core." For approximately the first 20 years following the first publications, only a modest amount of research was conducted concerning the helicon plasma source (Figure $1.1)$.

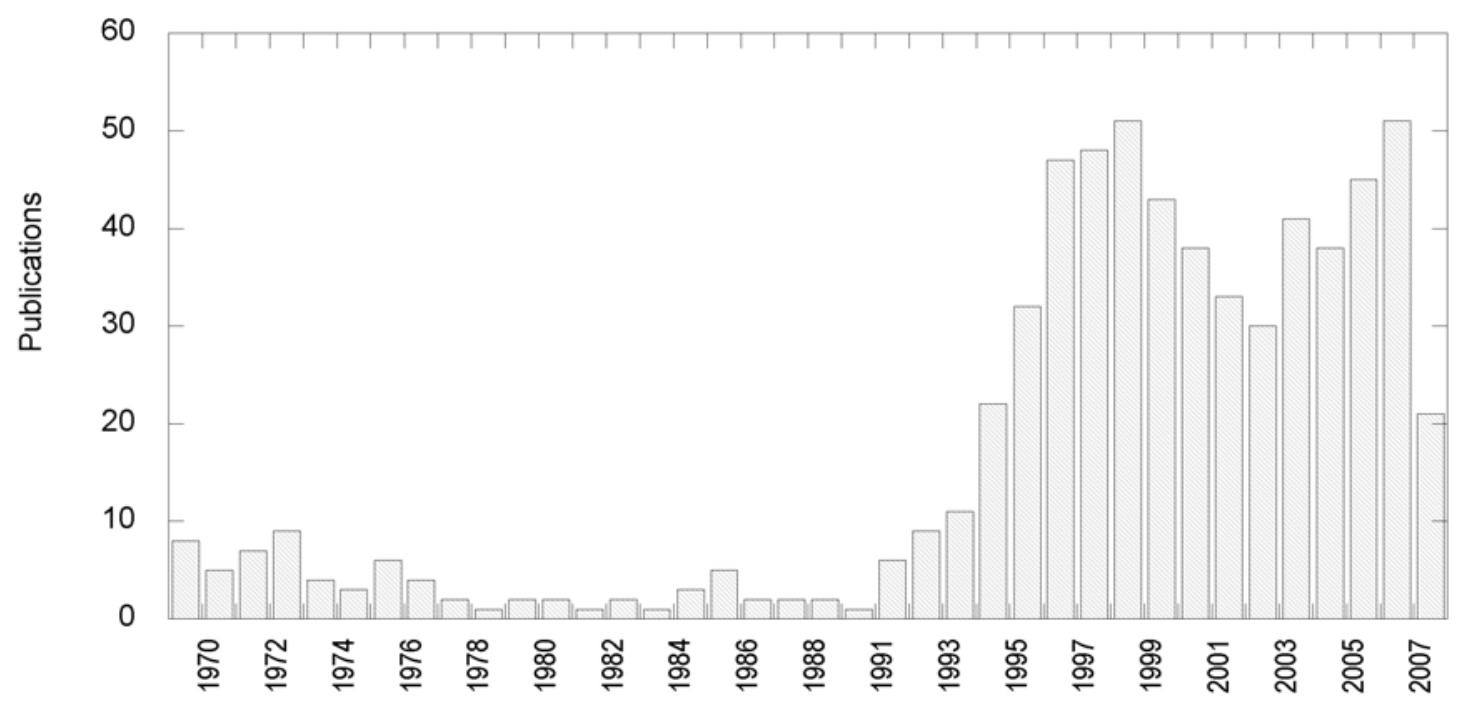

Figure 1.1 Journal publications each year including the terms "helicon plasma" in the title or abstract. ${ }^{3}$

Since the early 1990's, there has been a large increase in the number of publications related to the helicon source; largely due to the wide applicability of a plasma source with high density and low temperature. Helicon sources have been constructed for a variety of uses ranging from plasma thrusters, ${ }^{5,6,7,8}$ plasma processing, ${ }^{9,10}$ space relevant 
experiments, ${ }^{11,12}$ and basic plasma physics experiments. ${ }^{13,14}$ A helicon source is even being considered as a replacement for the current $\mathrm{H}^{-}$ion source for the Spallation Neutron Source at Oak Ridge National Laboratory. ${ }^{15}$ Since the initial helicon source experiment, over 600 journal articles that specifically refer to "helicon plasma" have appeared in the literature (Figure 1.1). ${ }^{3}$ After peaking in the late 1990s, the publication rate for "helicon source" literature currently averages about 30 journal articles each year. Excellent reviews are available on the early history of helicon research, including all the basic theory, by Boswell and Chen. ${ }^{1}$ Helicon research in the following 10 years was reviewed by Chen and Boswell. ${ }^{16}$ Most recently, a review by Scime, Keesee, and Boswell, following the mini-conference on helicon plasma sources at the $49^{\text {th }}$ Annual APS Division of Plasma Physics, discussed topics related to optimal source performance and novel applications of the helicon source. ${ }^{3}$

\subsection{The Helicon Plasma}

The helicon wave is a bounded right-hand circularly polarized electromagnetic wave, propagating in the frequency range $\omega_{c i} \ll \omega \ll \omega_{c e}$, where $\omega_{c i}$ is the ion cyclotron frequency, $\omega_{c e}$ is the electron cyclotron frequency, and $\omega$ is the wave frequency. Free (unbounded) right-hand circularly polarized electromagnetic waves are typically referred to as "whistler" waves because of their characteristic descending tones as heard during the later half of World War I. ${ }^{1}$ The waves were inadvertently picked up by radio communication spies while listening for enemy communications and were later determined to be an atmospheric phenomenon; initiated by lightening strikes generating the waves which then propagated along the magnetic field lines of the Earth. 
The term helicon was originally coined by Aigrain in 1960 to describe the propagation of bounded right hand circularly polarized waves in a solid rod of sodium. ${ }^{17}$ The dispersion relation for the helicon wave is:

$$
N^{2} \approx \frac{\omega_{p e}^{2}}{\omega \omega_{c e} \cos \theta},
$$

where $N$ is the parallel index of refraction, defined as $N=k_{\|} c / \omega, k_{\|}$is the wave number parallel to the magnetic field, $c$ is the speed of light, $\omega$ is the wave frequency, $\omega_{p e}$ is the electron plasma frequency, $\omega_{c e}$ is the electron cyclotron frequency, and $\theta$ is the angle at which the wave propagates with respect to the magnetic field. One interesting feature of the helicon dispersion relation is that the waves' maximum group velocity is $(d \omega / d k)_{\max }=\omega / 4 \omega_{c e} \cdot{ }^{18}$ Therefore, high frequency helicon waves travel faster, arriving earlier than low frequency waves emanating from the same source. This dispersion gives rise to the same "whistling" effect characteristic of the unbounded whistler waves recorded by the listening stations in the early part of the $20^{\text {th }}$ century.

One of the characteristic features associated with helicon source operation are discontinuous jumps in the density as the magnetic field is increased (Figure 1.2).${ }^{19}$ Note that the overall trend follows the simple helicon dispersion relation (dashed line in Figure 1.2) of Equation (1.1). For a fixed parallel wavelength twice that of the antenna ( $\lambda=50$ $\mathrm{cm}$ ), and substitution of other constants, Equation (1.1) reduces to a linear relationship between the density and magnetic field

$$
n \sim 1.2 \times 10^{9} B_{0} \mathrm{~cm}^{-3} .
$$

The density jumps, also referred to as "mode hops," are generally associated with specific operational modes of the source: the capacitive mode, the inductive mode, and 
the helicon mode. In the capacitive and inductive mode, the penetration of the fields into the plasma interior is limited to the skin depth, thus the power is deposited in the plasma edge. ${ }^{20}$ Because of the limited penetration depth, the capacitive and inductive modes are generally limited to densities on the order of $10^{10} \mathrm{~cm}^{-3}$ (lowest density of Fig 1.2) and $10^{11} \mathrm{~cm}^{-3}$ (middle density of Figure 1.2). In the helicon mode, penetration of the fields to the plasma interior results in more power deposition and more efficient density production, generally on the order of $10^{13} \mathrm{~cm}^{-3}$.

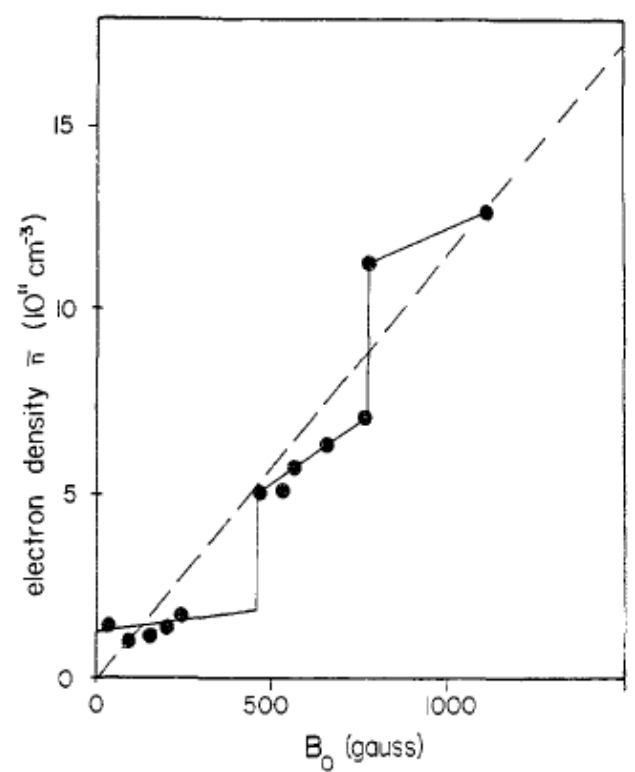

Figure 1.2 Density as a function of magnetic field, showing the density jumps associated with a helicon plasma source. Figure obtained from Ref. [19].

Experiments examining other external source parameters, such as the applied rf power (Figure 1.3) ${ }^{21}$ and background neutral pressure (Figure 1.4), ${ }^{22}$ have shown the same "mode hop" transitions in density characteristic of the helicon source. Figure 1.3 shows how the density varies as a function of applied rf power for several magnetic field strengths. For magnetic fields below 400 Gauss, the density increases, but no mode transitions are observed. Magnetic fields of 500 Gauss and larger display mode transitions, and as the field is increased to 1000 Gauss, the density jumps occur at lower 
applied rf powers. Note that although the jumps may occur at lower rf power as the field is increased, the maximum density achieved is slightly lower than $10^{13} \mathrm{~cm}^{-3}$. Figure 1.4 shows how the density varies as a function of $\mathrm{rf}$ driving frequencies for different neutral pressures. Again, the density jumps are present as well as the similar trend of a shifting density threshold. In this case, the density jump shifts to lower rf frequencies as the neutral density is increased.

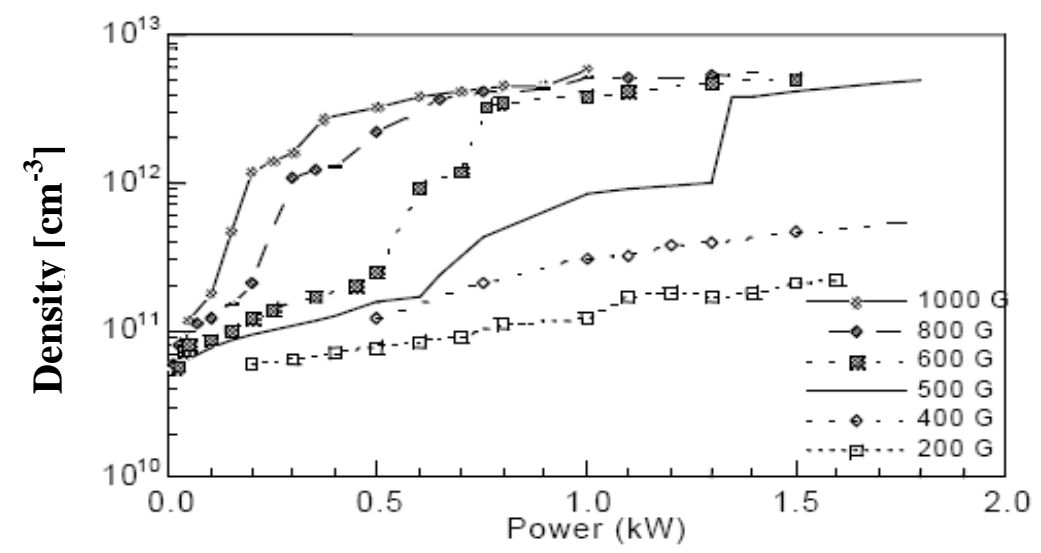

Figure 1.3 Density as a function of applied rf power for varying magnetic field strengths. Figure obtained from Ref. [21].

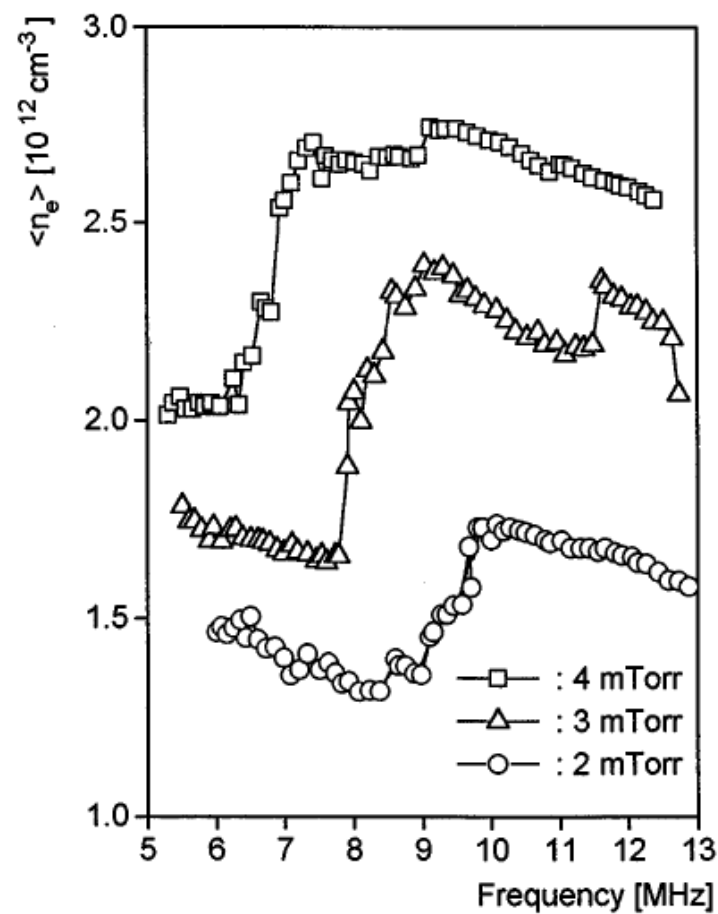

Figure 1.4 Density as a function of frequency for varying neutral gas pressures for an applied rf power of $1.2 \mathrm{~kW}$ and magnetic field of $800 \mathrm{G}$. Figure obtained from Ref. [22]. 
Although researchers often turn to helicon sources for efficient plasma production and high densities, the exact mechanism responsible for coupling the rf power into the plasma is not completely understood. While the majority of helicon source researchers are not focused on investigating the mechanisms responsible for the rf power coupling, several mechanisms have been suggested and examined over the years. One of the first suggestions was that the rf coupling could be explained through either collisional damping of the helicon wave or Landau damping of the helicon wave on the electrons. ${ }^{23}$ Calculations determined that the collisional damping of the helicon wave, particularly for low neutral pressures, was insufficient to explain the power coupling. ${ }^{23}$ As for Landau damping of the helicon wave on the electrons, there needs to be enough of an energetic electron population for the damping to play a significant role. Measurements that hinted of energetic electron populations sufficient to contribute to the Landau damping process were reported, ${ }^{24,25,26}$ but subsequent measurements found that the population of energetic electrons was too sparse in the helicon source to make a significant contribution to the power deposition. ${ }^{27}$ It should be noted that in the same work, the measured resistive loading on the rf antenna was consistent with coupling to electrostatic waves in the plasma edge. Those measurements, which can be used as a proxy for the coupling efficiency of the antenna to the excitation of electrostatic waves, suggested that electrostatic waves were being excited and because of their short wavelength nature were strongly absorbed as they propagate inward. Another example where the resistive loading on a wave launching antenna was used to gauge the coupling efficiency of electrostatic waves was by Takase et al. on the Alcator-C tokamak for the launching of ion Bernstein waves. ${ }^{28}$ Currently one of the leading mechanisms being considered for coupling of the rf 
power into helicon plasmas is the damping of short wavelength electrostatic waves in the plasma edge when operating near the lower hybrid frequency.

\subsection{The Lower Hybrid Wave Resonance and the Slow Wave}

The electrostatic slow wave, often referred to as the "Trivelpiece-Gould" (TG) wave, ${ }^{29}$ is believed by some to play a key role in the high rf absorption efficiency of helicon sources operating near the lower hybrid frequency. ${ }^{30,31,32,33}$ The lower hybrid frequency is defined as

$$
\frac{1}{\omega_{L H}^{2}}=\frac{1}{\omega_{c e} \omega_{c i}}+\frac{1}{\omega_{p i}^{2}+\omega_{c i}^{2}}
$$

where $\omega_{L H}$ is the lower hybrid frequency, $\omega_{c e}$ and $\omega_{c i}$ are the electron and ion cyclotron frequencies and $\omega_{p i}$ is the ion plasma frequency. Typically in helicon sources, $\omega_{p i} \gg \omega_{c i}$, resulting in

$$
\frac{1}{\omega_{L H}^{2}}=\frac{1}{\omega_{c e} \omega_{c i}}+\frac{1}{\omega_{p i}^{2}}
$$

Because helicon sources have peak axial densities on the order of $10^{13} \mathrm{~cm}^{-3}$, Equation (1.4) can be simplified further yielding

$$
\omega_{L H} \cong \sqrt{\omega_{c e} \omega_{c i}} .
$$

Since in a typical helicon source, the density at the edge decreases by approximately an order of magnitude relative to the density on axis, the term containing $\omega_{p i}$ in Equation (1.4) must be considered when calculating the lower hybrid frequency throughout the plasma. The inclusion of the ion plasma frequency term reduces the lower hybrid frequency in the lower density plasma edge. 
The reason the slow wave is referred to as the TG wave in the helicon literature is because it corresponds to the same root of the cold plasma dispersion function that was identified by Trivelpiece and Gould for a bounded, pure electron plasma. ${ }^{29}$ Several groups have predicted through computation that the rf power is absorbed more efficiently by the TG waves than by the helicon wave. ${ }^{34,35,36,37,38,39}$ Recently Blackwell et al. reported experimental evidence for the TG mode in a helicon source through measurements of the parallel rf current. ${ }^{40}$ An important aspect of the Blackwell et al. measurements is that the magnetic field was restricted to $25-60 \mathrm{G}$, while most helicon sources operate at magnetic fields in the hundreds of Gauss. With that in mind, their experiment with an $\mathrm{rf}$ driving frequency of $11 \mathrm{MHz}$, densities of $\sim 5 \times 10^{11} \mathrm{~cm}^{-3}$, and magnetic fields of $60 \mathrm{G}$, operated well above the lower hybrid frequency, where TG mode can still propagate but no lower hybrid resonance effects are expected.

Typical helicon plasma sources operate at only a few rf frequencies and most sources operate at a single frequency. The rf frequencies used for helicon sources generally range between $5 \mathrm{MHz}$ and $28 \mathrm{MHz},{ }^{19,21,22,41,42}$ but some groups have operated helicon sources at frequencies as high $144 \mathrm{MHz}{ }^{43}$ The limited range of source rf frequencies prevents most helicon source groups from exploring possible lower hybrid resonance effects and slow wave damping. One of the unique features of the WVU helicon plasma source is the ability to vary the rf frequency between 6 and $18 \mathrm{MHz}$, allowing for an extensive study of the lower hybrid resonance and the possible excitation of slow waves.

Early experiments at WVU, designed to maximize density production and minimize intrinsic ion heating via different antennas, indicated that the lower hybrid frequency played an important role in the source operation. ${ }^{44}$ Figure 1.5 shows the measured 
perpendicular ion temperature, electron density, and electron temperature as a function of magnetic field and rf driving frequency for four different antennas. The principle feature to note is that the largest electron density production occurs when the rf frequency is larger than the on axis lower hybrid frequency, denoted by the white line where $\omega \approx \omega_{L H} \cong \sqrt{\omega_{c e} \omega_{c i}}$, while the largest ion temperature occurs when the rf frequency is smaller than the lower hybrid frequency. The vertical dashed lines are at the conventional 13.56 MHz rf frequency. For other helicon sources with similar operating parameters (magnetic field, pressure, etc.), lower hybrid frequency resonance effects would be minimal if the source was operated at frequencies at or above $13.56 \mathrm{MHz}$. 


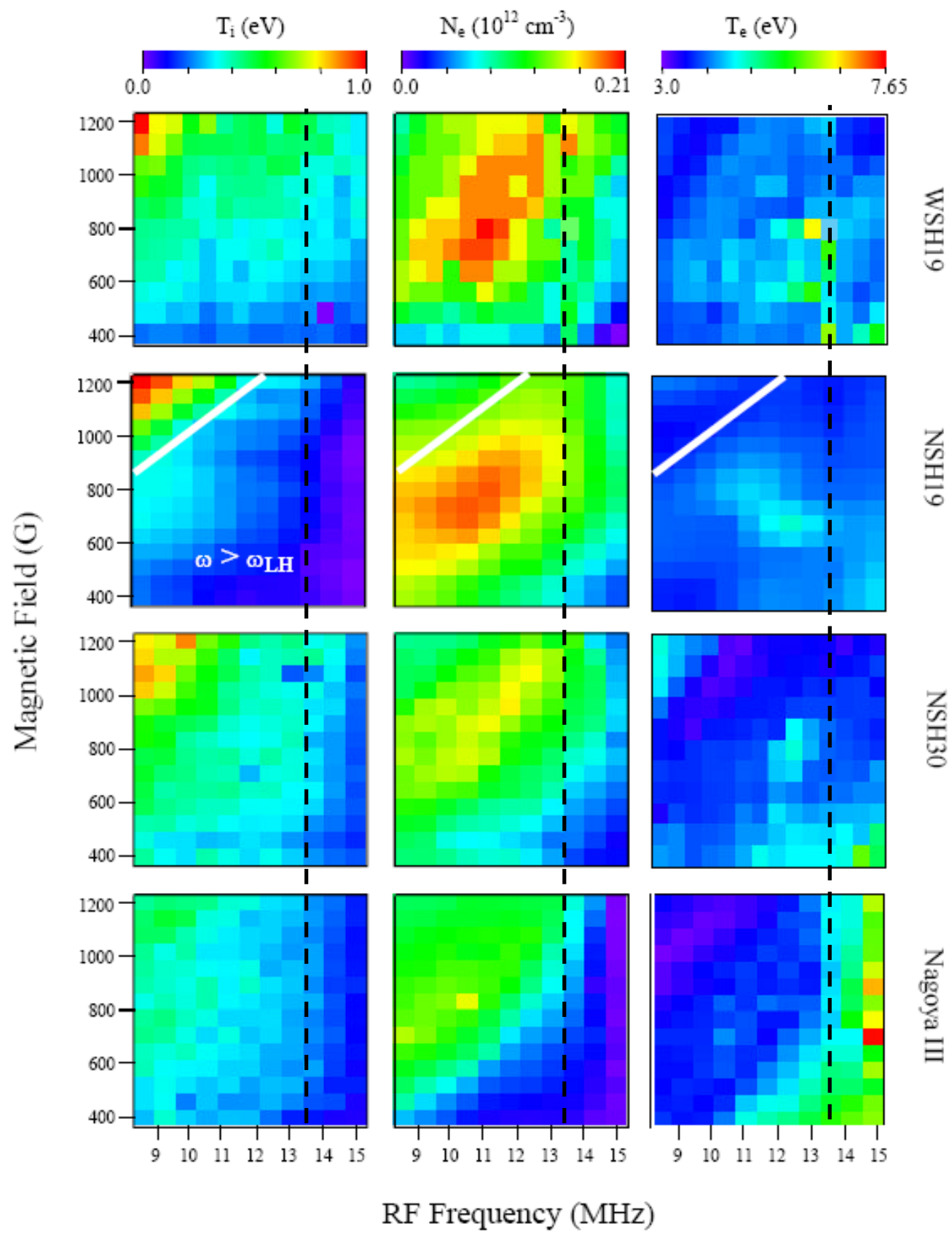

Figure 1.5 Perpendicular ion temperature, electron density, and electron temperature as a function of magnetic field and $\mathrm{rf}$ driving frequency for four antennas (See Ref. 45 for antenna details). Operating parameters for all measurements were a neutral pressure of 3.6 mTorr and rf power of $750 \mathrm{~W}$. Figure obtained from Ref. [45].

Recent experiments examining the perpendicular and parallel ion temperature as a function of plasma radius (Figure 1.6) showed an ion temperature increase near the plasma edge. ${ }^{46}$ It's important to note that the perpendicular ion temperature increased 
preferentially at the plasma edge, while the parallel temperature tended to decrease near the edge. More comprehensive experiments (Figure 1.7b), clearly demonstrated an increase in the perpendicular ion temperatures when the rf frequency is lower than the lower hybrid frequency. The white line in Figure 1.7b denotes the lower hybrid frequency calculated on axis, while the arrow points in the direction the lower hybrid frequency would shift for lower densities, such as near the plasma edge.

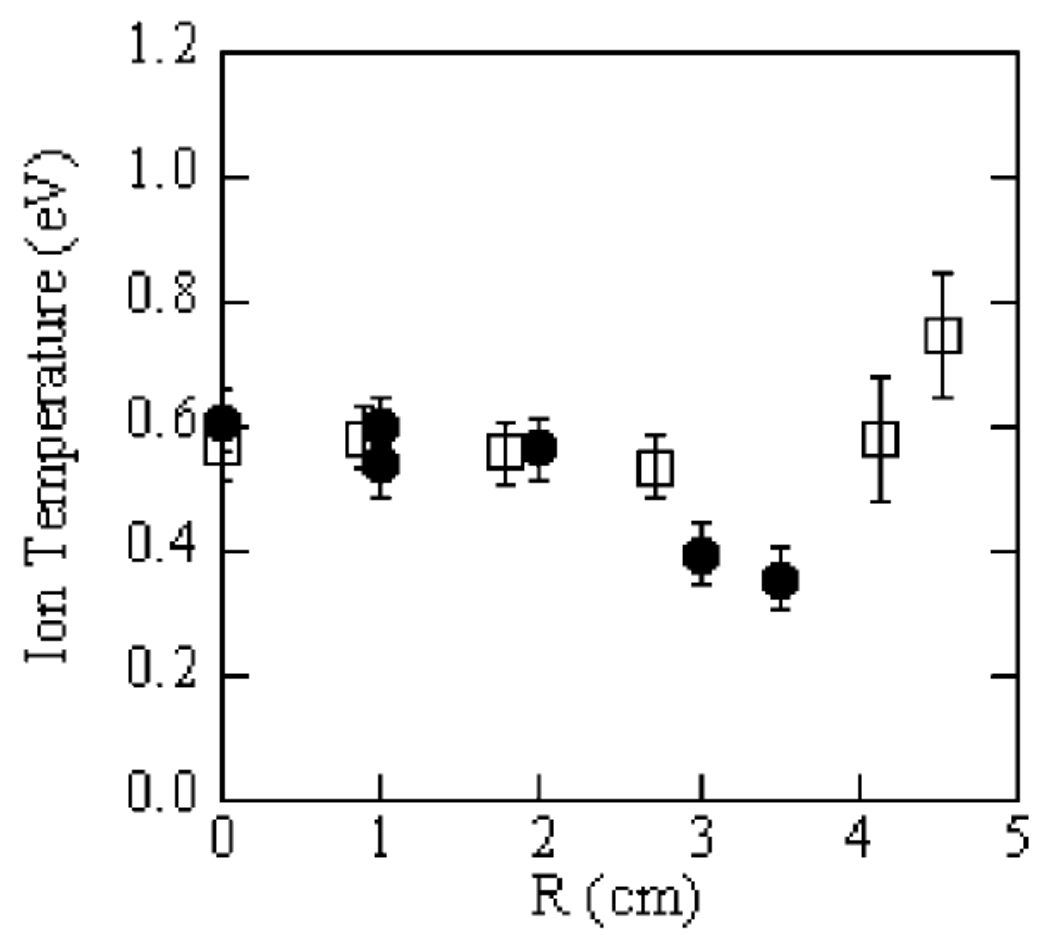

Figure 1.6 (प) Perpendicular and ( $(\bullet)$ parallel ion temperatures, measured with LIF, as a function of radius for a magnetic field of $1200 \mathrm{G}$, neutral pressure of $6.7 \mathrm{mTorr}$, rf frequency of $9 \mathrm{MHz}$, and rf power of 750 W. Figure obtained from Ref. [46].

The normalized perpendicular wave numbers $\left(k_{\perp} v_{\text {thi }} / \omega\right)$ of the slow wave (Figure 1.7a), as calculated by the cold plasma dispersion function (discussed in Chapter 4), are largest when the rf frequency is just below the axial lower hybrid frequency. This suggests that near the lower hybrid resonance $\left(\omega \approx \omega_{L H}\right)$, where the perpendicular wave numbers are large, the phase speed of the wave is reduced enough that ion Landau 
damping could occur. The calculations shown in Figure 1.7a, for an ion temperature of $0.2 \mathrm{eV}$, indicate that the phase velocity drops to approximately a factor of 5 above the ion thermal velocity for rf frequencies just below the axial lower hybrid frequency. Assuming the slow wave Landau damps on the ions near the edge, damping of the slow wave can explain the preferential heating of the ions in the perpendicular direction. Thus the correlation between the largest calculated normalized wave numbers and measured ion temperatures shown in Figure 1.7 provides indirect evidence that the slow wave exists in helicon plasmas and is responsible, through damping, for the ion heating in the edge.
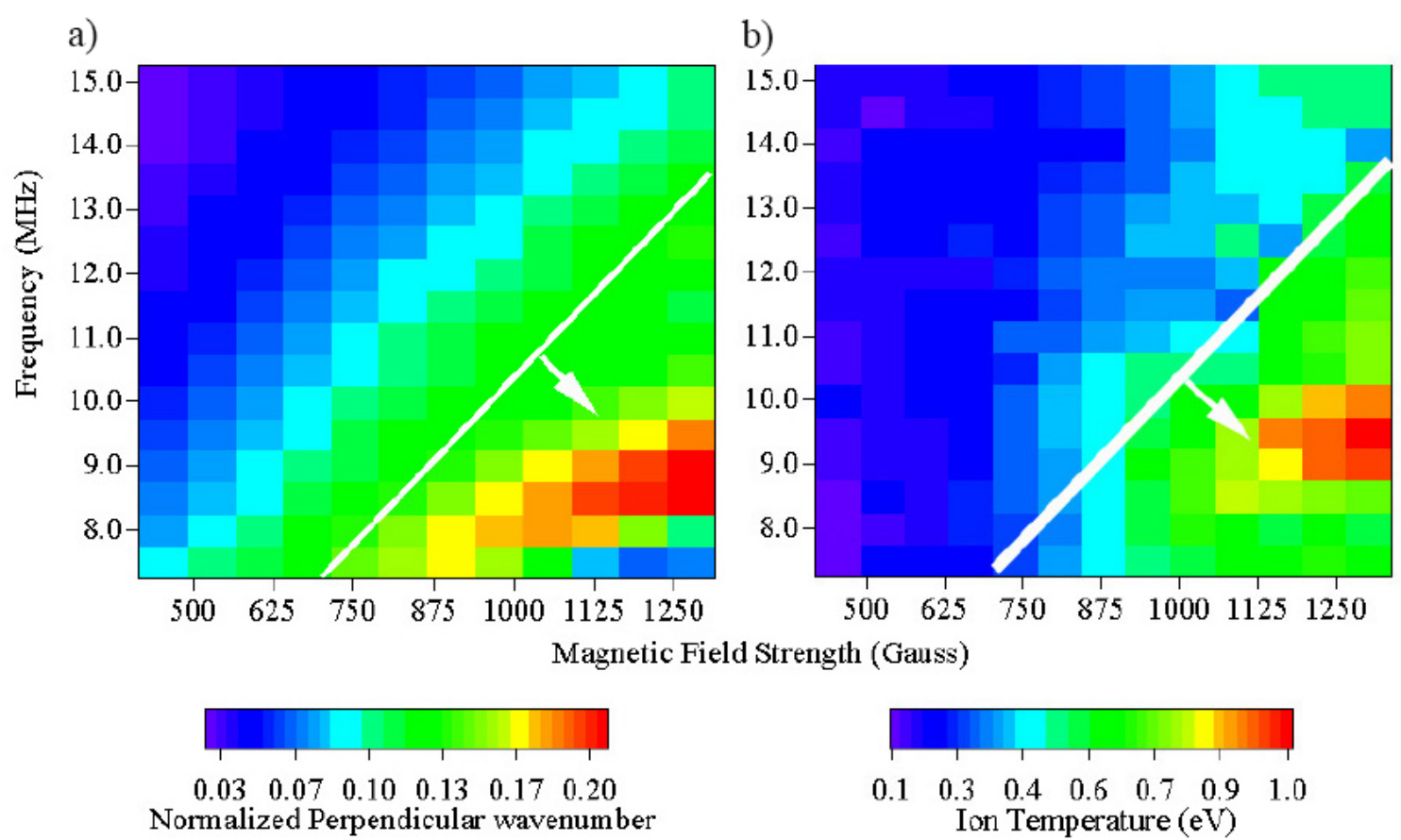

$$
\text { Normalized Perpendicular wavenumber }
$$

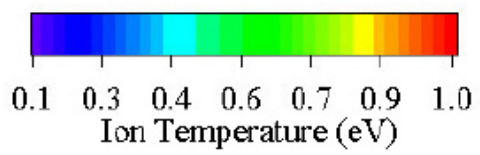

Figure 1.7 a) Normalized wave numbers, $k_{\perp} v_{\text {thi }} / \omega$, as calculated from the slow wave model. b) Ion temperatures measured via laser induced fluorescence in HELIX. The white line indicates where the rf driving frequency is equal to the on axis lower hybrid frequency, and the arrow points in the direction the line would shift for lower plasma densities as at the plasma edge. Operational parameters are a neutral pressure of 6.7 mTorr and an rf power of $750 \mathrm{~W}$. Figure obtained from Ref. [46].

Of course the best way to demonstrate that the slow wave is excited at the parameters for which the perpendicular ion temperature is large, at $\omega \approx \omega_{L H}$ in the plasma edge is to directly detect short wavelength electrostatic waves in the plasma edge. The problem 
with measuring the slow wave, especially if the wave number is large enough to produce ion Landau damping, is that the expected wavelengths are on the order of $1 \mathrm{~mm}$ or smaller. Previous measurements of ion-acoustic and lower hybrid waves with an electrostatic double probe in HELIX were limited to wave numbers $\leq 9.8 \mathrm{rad} / \mathrm{cm}(\lambda \geq$ $6.4 \mathrm{~mm}) \cdot{ }^{47}$ Measuring waves on the order of $1 \mathrm{~mm}$ or smaller is problematic with typical probes because the probes need to be very small and are therefore unlikely to survive the extreme plasma environment of a helicon source. The limited measureable range and survivability of standard probes, as well as the large wave numbers expected near the lower hybrid resonance, requires another measurement technique.

\subsection{Fluctuation Measurement by Collective Thomson Scattering}

With the limited applicability of standard probe techniques for the measurement of short wavelength fluctuations in plasma to study transport phenomena and resonance heating mechanisms, particularly in the fusion community, other methods to measure small scale fluctuations have been developed. The introduction of the first laser, producing a stable monochromatic source of radiation, ${ }^{48}$ paved the way for the development of a plethora of laser based plasma diagnostics, including Collective Thomson scattering. Collective Thomson scattering (CTS) has been employed as a plasma diagnostic since Surko et al. introduced the technique to measure cyclotronharmonic waves in a simple plasma device. ${ }^{49}$ Soon after, this non-invasive technique was adopted by the fusion community because of its ability to measure coherent fluctuations in plasmas where physical probes cannot survive. One of the first reported measurements employed a $200 \mathrm{~W}, 10.6 \mu \mathrm{m}$ continuous wave (cw) laser to observe electrostatic density 
fluctuations in the Adiabatic Toroidal Compressor tokamak. ${ }^{50}$ Over the years, CTS has been used to measure an assortment of fluctuations, including electron plasma waves, ${ }^{51}$ ion acoustic waves, ${ }^{52,53}$ ion Bernstein waves, ${ }^{54}$ lower hybrid waves, ${ }^{55,56}$ and even turbulence. ${ }^{57,58}$ Measurement of electrostatic fluctuations can play a significant role in improving the understanding of the physics of transport phenomena and resonance heating mechanisms.

When a monochromatic beam of radiation with frequency $\omega_{0}$ and wave number $k_{0}$, is incident upon a coherent fluctuation with frequency $\omega$ and wave number $k$, the scattered electromagnetic wave $\left(\omega_{s}, k_{s}\right)$ must satisfy energy and momentum conservation

$$
\omega_{s}=\omega_{0} \pm \omega
$$

and

$$
\vec{k}_{s}=\vec{k}_{0} \pm \vec{k}
$$

respectively. Since for most laboratory plasmas $\omega \ll \omega_{0}$, while simultaneously satisfying $\omega_{0} \gg \omega_{p e}$, the scattered radiation is peaked about the scattering angle $\left(\theta_{s}\right)$ given by the Bragg condition

$$
k=2 k_{0} \sin \left(\theta_{s} / 2\right)
$$

Equation 1.8 provides a means of estimating the range of fluctuation wave numbers measureable by CTS, based simply on the incident wave number and the observable scattering angles. The scattering from plasma fluctuations satisfying the condition

$$
k \lambda_{\text {De }}<1
$$


where $\lambda_{D e}$ is the electron Debye length, is defined as coherent scattering. The scattered radiation will experience a Doppler shift proportional to the phase velocity of the fluctuation along the direction of propagation, given by

$$
\omega=\omega_{s}-\omega_{0}=v_{p}|\vec{k}|
$$

where $v_{p}$ is the phase velocity of the wave. ${ }^{60} \mathrm{~A}$ schematic showing the general geometry for CTS is shown in Figure 1.8.

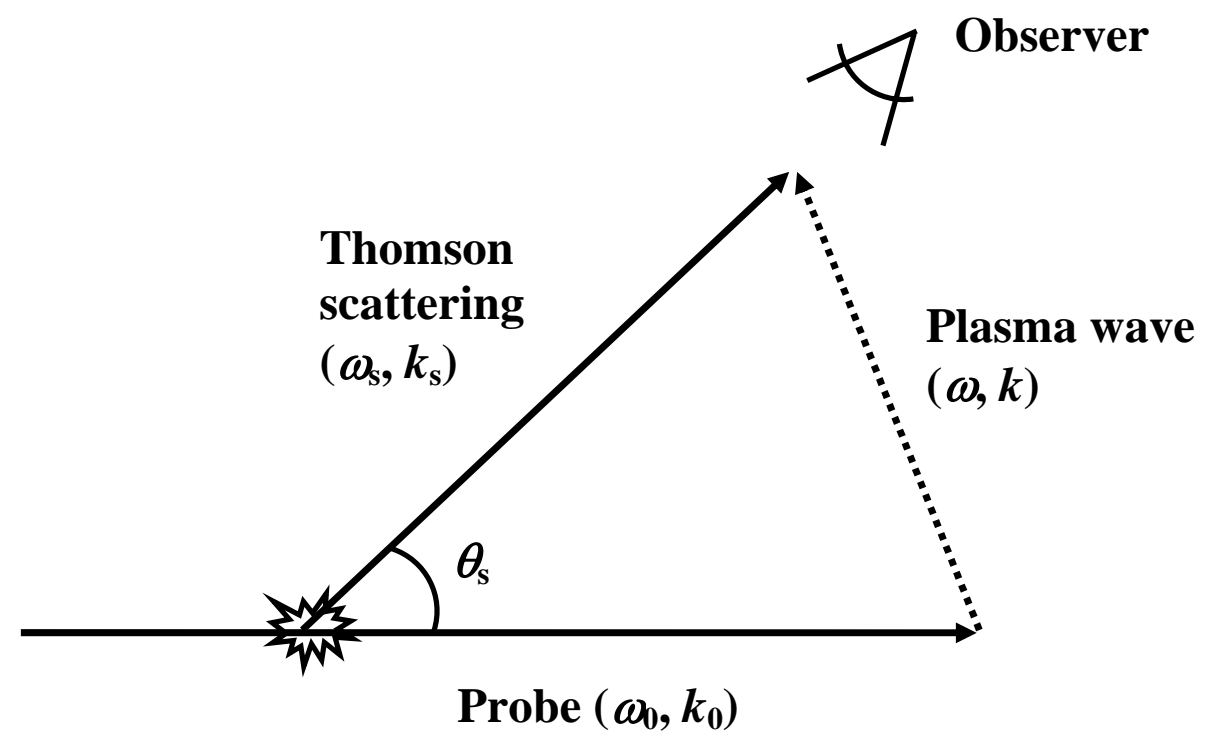

Figure 1.8 Schematic diagram of collective Thomson scattering geometry.

The amount of scattered power, in Watts, from a coherent fluctuation measured with CTS is given by

$$
P_{s}=\frac{1}{4} P_{0} r_{e}^{2} \lambda_{0}^{2} L_{v}^{2}(\tilde{n})^{2}
$$

where $P_{0}$ is the incident beam power, $r_{e}$ is the classical electron radius, $\lambda_{0}$ is the incident wavelength, $L_{v}$ is the length of the scattering volume, and $\tilde{n}$ is the density fluctuation amplitude. ${ }^{59}$ The dependence of the scattered power on the square of the incident 
wavelength is another important consideration when designing a CTS diagnostic. For example, for similar scattering volumes and fluctuation amplitudes, a CTS system with a laser wavelength of $527 \mathrm{~nm}$ would require approximately 400 times more incident power to produce the same amount of scattered power as a $10.6 \mu \mathrm{m} \mathrm{CO}_{2}$ laser based CTS system. An example of CTS scattered power as a function of wave number and frequency is shown in Figure 1.9 from the $119 \mu \mathrm{m}$ based CTS diagnostic used on the ASDEX tokamak. The dominant feature in Figure 1.9 is the broadband peak in the signal power around a frequency of $100 \mathrm{kHz}$ and wave number of $5 \mathrm{~cm}^{-1}$, which is a characteristic feature of turbulence observed in many tokamaks. ${ }^{60}$

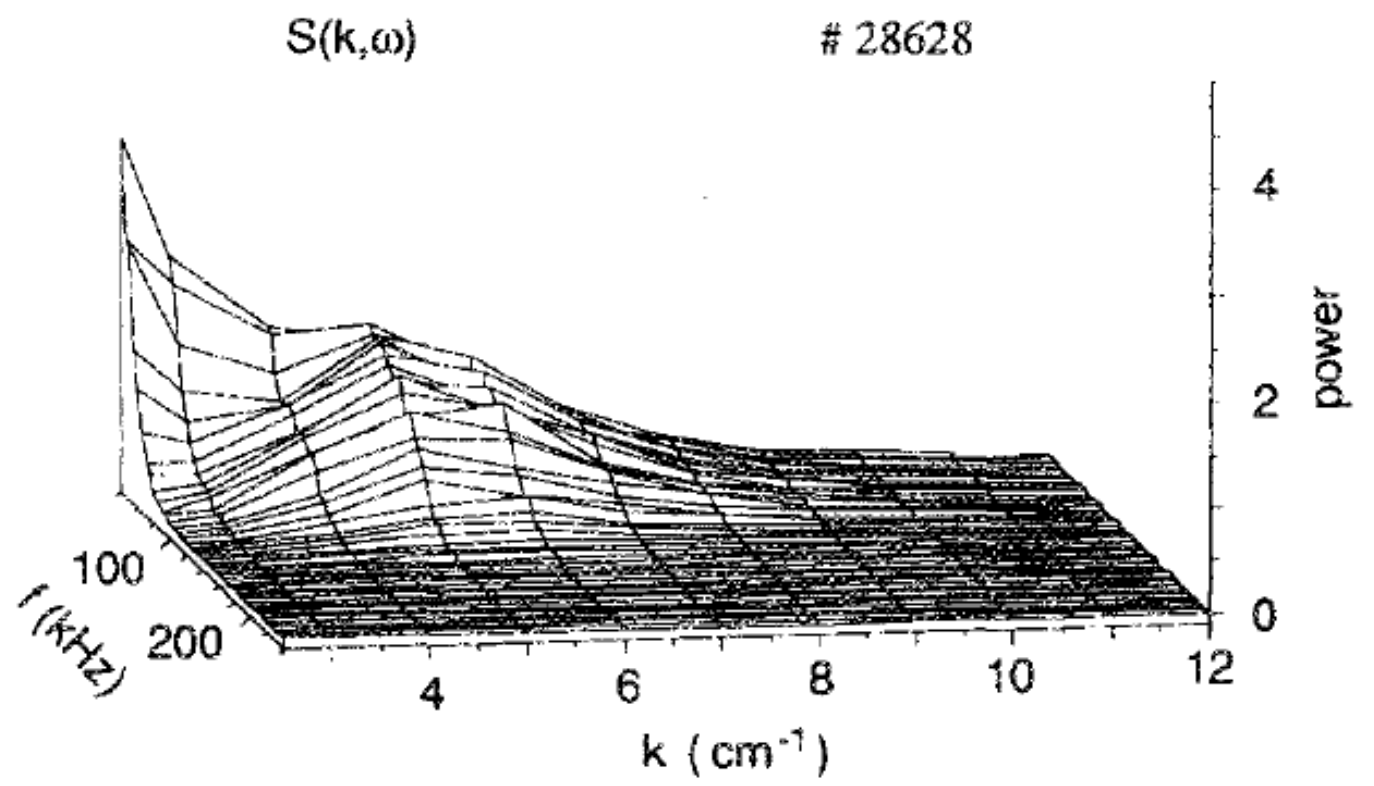

Figure 1.9 Scattered signal power as a function of wave number and frequency from the ASDEX FIR CTS diagnostic. Figure obtained from Ref. [60].

Although CTS has been used almost exclusively as a fusion plasma diagnostic, measurements employing CTS in helicon sources have begun to appear due to the effectiveness of the technique. ${ }^{61,62,63,64}$ Using a technique to enhance the scattered signal by scattering off the upper hybrid resonance layer, $\omega_{U H}^{2} \equiv \omega_{p e}^{2}+\omega_{c e}^{2}$, both ion-acoustic and 
TG modes (lower hybrid waves) have been observed propagating radially in a helicon plasma source (see Figure 1.10). The diagnostic used two microwave sources at 9 and 28 $\mathrm{GHz}$ with output powers of $50 \mathrm{~mW}$ and $20 \mathrm{~mW}$, respectively. ${ }^{64,62}$ Although the measurement indicated that both ion-acoustic and lower hybrid waves were present, the helicon source operated at an rf frequency of $13.56 \mathrm{MHz}$, where no significant lower hybrid resonance effects were expected. With that in mind, note that both waves exhibit wave number magnitudes approaching $\sim 75 \mathrm{rad} / \mathrm{cm}$. Although the diagnostic had a maximum wave number range of approximately $200 \mathrm{rad} / \mathrm{cm}$, the detection sensitivity at the largest wave numbers was not reported. Thus, it is possible that significant wave power may have existed at wave numbers larger than the $75 \mathrm{rad} / \mathrm{cm}$ shown in Figure 1.10. Note also that significant scattered power was observed at low frequencies and wave numbers of $\sim 75 \mathrm{~cm}^{-1}$ (bottom panel of Figure 1.10).

An example of using a CTS diagnostic to measure ion acoustic waves in a helicon source is shown in Figure 1.11. ${ }^{63}$ The importance of this measurement is not that the ionacoustic wave was measured in a helicon source, but that a CTS diagnostic employing a $140 \mathrm{GHz}\left(\lambda_{0} \sim 2 \mathrm{~mm}\right)$ source with only $10 \mathrm{~mW}$ of output power was used to obtain the measurement. This is an illustration of the importance of the wavelength dependence in Equation 1.11 for a CTS diagnostic. 


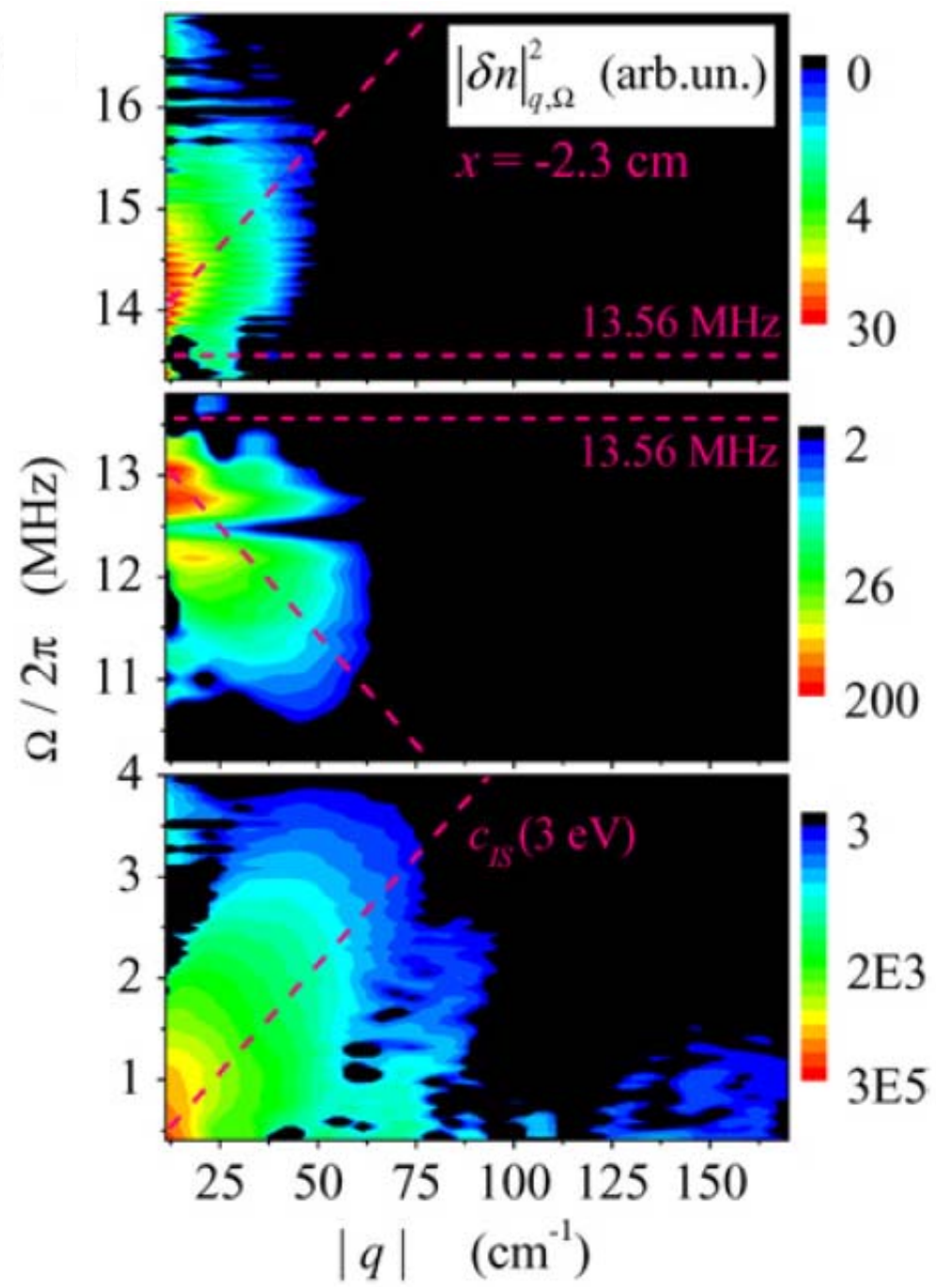

Figure 1.10 Enhanced CTS measured density fluctuation signals as a function of wave number $(q)$ and frequency $(\Omega / 2 \pi)$ of ion-acoustic $(\Omega / 2 \pi<4 \mathrm{MHz}$ region) and lower hybrid $(10<\Omega / 2 \pi<14 \mathrm{MHz}$ region) waves from the pulsed helicon source HE-L. Figure obtained from Ref. [64]. 


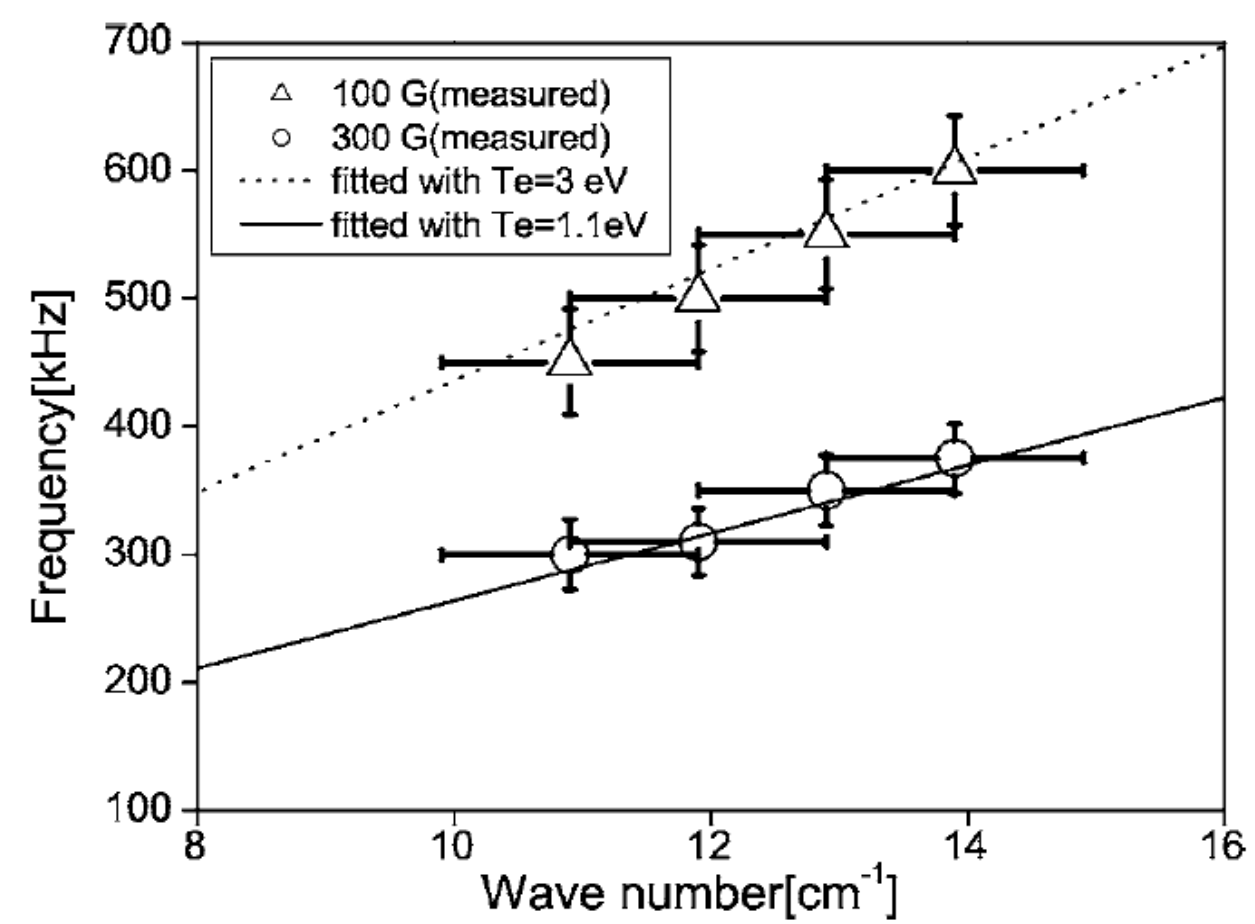

Figure 1.11 Ion-acoustic wave dispersion curves obtained by CTS in a helicon plasma source. Figure obtained from Ref. [63].

One drawback to the CTS diagnostics used to obtain the results shown in Figure 1.10 and Figure 1.11 is their limited wave number ranges. Assuming the maximum measureable wave number in Figure 1.10 is limited to $\sim 75 \mathrm{rad} / \mathrm{cm}$ and calculating the maximum measureable wave number for the system in Figure 1.11 to be $\sim 61 \mathrm{rad} / \mathrm{cm}$ (see Equation 1.8), the wave number ranges for both systems are too small to detect slow waves with wave numbers large enough to be Landau damped by ions. With the slow wave expected to be spatially localized to the plasma edge and given the large wave numbers needed for Landau damping of the slow wave on the ions, the ability to make spatially localized fluctuation measurements with a CTS diagnostic capable of larger wave number measurements is required for the detection and investigation of the slow wave in a helicon plasma source. Because the WVU helicon plasma source has been shown to have operational parameters capable of producing plasmas with frequencies 
above and below the lower hybrid frequency, it is ideally suited for the development of a new CTS diagnostic capable of larger wave number measurements at frequencies likely to provide evidence of lower hybrid resonance effects. 


\section{Chapter 1 References}

${ }^{1}$ R.W. Boswell, A Study of Wave in Gaseous Plasmas, Ph.D. Thesis, Flinders University, Adelaida, Australia (1974).

${ }^{2}$ R.W. Boswell and F.F. Chen, IEEE Trans. Plasma Sci., 25, 1229 (1997).

${ }^{3}$ E.E. Scime, A.M. Keesee, and R.W. Boswell, Phys. Plasmas, 15, 058301 (2008).

${ }^{4}$ R.W. Boswell, Phys. Lett., 33, 457 (1970).

${ }^{5}$ J.P. Squire, F.R. Chang Diaz, T.W. Glover, V.T. Jacobson, D.G. Chavers, R.D. Bengston, E.A. Bering III, R.W. Boswell, R.H. Goulding, and M. Light, Fusion Sci. Technol., 43, 111 (2003).

${ }^{6}$ R. W. Boswell, O. Sutherland, C. Charles, J.P. Squire, F.R. Chang Diaz, T.W. Glover, V.T. Jacobson, D.G. Chavers, R.D. Bengston, E.A. Bering, R.H. Goulding, and M. Light, Phys. Plasmas, 11, 5125 (2004).

${ }^{7}$ X. Sun, C. Biloiu, R. Hardin, E.E. Scime, Plasma Sources Sci. Technol., 13, 359 (2004).

${ }^{8}$ R. Winglee, T. Ziemba, L. Giersch, J. Prager, J. Carscadden, and B.R. Roberson, Phys. Plasmas, 14, 63501 (2007).

${ }^{9}$ G.W. Gibson Jr. and D.J. Hemker, Semiconductor International, 19, 193 (1996).

${ }^{10}$ S. Kimura and H. Ikoma, J. Appl. Phys., 85, 551 (1999).

${ }^{11}$ E.E. Scime, P.A. Keiter, M.M. Balkey, R.F. Boivin, J.L. Kline, M. Blackburn, and S.P. Gary, Phys. Plasmas, 7, 2157 (2000).

${ }^{12}$ J. Hanna and C. Watts, Phys. Plasmas, 8, 4251 (2001).

${ }^{13}$ K. Itoh, S. Itoh, P.H. Diamond, T.S. Hahm, A. Fujisawa, G.R. Tynan, M. Yagi, and Y. Nagashima, Phys. Plasmas, 13, 55502-1 (2006).

${ }^{14}$ M.I. Panevsky and R.D. Bengston, Phys. Plasmas, 11, 4196 (2004).

${ }^{15}$ R.H. Goulding, F.W. Baity, D.A. Rasmussen, D.O. Sparks, M.D. Carter, and M. Yoshitaka, Bull. Am. Phys. Soc., 52, 139 (2007).

${ }^{16}$ F.F. Chen and R.W. Boswell, IEEE Trans. Plasma Sci., 25, 1245 (1997).

${ }^{17}$ P. Aigrain, Proc. Int. Conf. Semiconductor Physics, 224 (1960).

${ }^{18}$ L.R. Storey, Philos. Trans. R. Soc. London A, Math. Phys. Sci., 246, 113 (1953).

${ }^{19}$ R.W. Boswell, Plasma Phys. Control Fusion, 26, 1147 (1984).

${ }^{20}$ A.R. Ellingboe and R.W. Boswell, Phys. Plasmas, 3, 2797 (1996).

${ }^{21}$ P.A. Keiter, E.E. Scime, and M.M. Balkey, Phys. Plasmas, 4, 2741 (1997).

${ }^{22}$ J.G. Kwak, et. al., Phys. Plasmas, 4, 1463 (1997).

${ }^{23}$ F.F. Chen, Plasma Phys. Controlled Fusion, 33, 339 (1991).

${ }^{24}$ P. Zou and R.W. Boswell, J. of Appl. Physics, 68, 1981 (1990).

${ }^{25}$ A.R. Ellingboe, R.W. Boswell, J.P. Booth, and N. Sadeghi, Phys. Plasmas, 2, 1807 (1995).

${ }^{26}$ R.T.S. Chen and N. Hershkowitz, Phys. Rev. Lett., 80, 4677 (1998).

${ }^{27}$ F.F. Chen and D.D. Blackwell, Phys. Rev. Lett., 82, 2677 (1999).

${ }^{28}$ Y. Takase, J.D. Moody, C.L. Fiore, F.S. McDermott, M. Porkolab, and J. Squire, Phys. Rev. Lett., 59, 1201 (1987).

${ }^{29}$ A.W. Trivelpiece and R.W. Gould, J. Appl. Physics 17, 1784 (1959).

${ }^{30}$ K.P. Shamrai and V.B. Taranov, Phys. Lett. A, 204, 139 (1995).

${ }^{31}$ K.P. Shamrai and V.B. Taranov, Plasma Sources Sci. Technol., 5, 474 (1996).

${ }^{32}$ G.G. Borg and R.W. Boswell, Phys. Plasmas, 5, 564 (1998).

${ }^{33}$ D. Arnush, Phys. Plasmas, 7, 3024 (2000).

${ }^{34}$ I.V. Kamenski and G.G. Borg, Comput. Phys. Commun., 113, 10 (1998).

${ }^{35}$ T. Enk and M. Kramer, Phys. Plasmas, 7, 4308 (2000).

${ }^{36}$ Y. Mouzouris and J.E. Scharer, Phys. Plasmas, 5, 4253 (1998).

${ }^{37}$ B.H. Park, N.S. Yoon, and D.I. Choi, IEEE Trans. Plasma Sci., 29, 502 (2001).

${ }^{38}$ K.P. Shamrai and S. Shinohara, Phys. Plasmas, 8, 4659 (2001).

39 A. Ganguli, B.B. Sahu, and R.D. Tarey, Phys. Plasmas, 14, 113503 (2007).

${ }^{40}$ D.D. Blackwell, T.G. Madziwa, D. Arnush, and F.F. Chen, Phys. Rev. Lett., 88, 145002 (2002).

${ }^{41}$ F.F. Chen, J. Vac. Sci. Technol. A, 10, 1389 (1992). 
${ }^{42}$ S. Yun, J.H. Kim, and H.Y. Chang, J. Vac. Sci. Technol. A, 15, 673 (1997).

${ }^{43}$ Y. Sakawa, H. Kunimatsu, H. Kikuchi, Y. Kukui, and T. Shoji, Phys. Rev. Lett., 90, 105001 (2003).

${ }^{44}$ M.M. Balkey, R. Boivin, J.L. Kline, and E.E. Scime, Plasma Sources Sci. Technol., 10, 284 (2001).

${ }^{45}$ M.M. Balkey, Optimization of a Helicon Plasma Source for Maximum Density with Minimal Ion Heating, Ph.D. Dissertation, West Virginia University, Morgantown (2000).

${ }^{46}$ J.L. Kline, E.E. Scime, R.F. Boivin, A.M. Keesee, X. Sun, and V.S. Mikhailenko, Phys. Rev. Lett., 88, $195002(2002)$.

${ }^{47}$ J.L. Kline and E.E. Scime, Phys. Plasmas, 10, 135 (2003).

${ }^{48}$ T.H. Maiman, Nature, 187, 493 (1960).

${ }^{49}$ C.M. Surko, R.E. Slusher, D.R. Moler, and M. Porkolab, Phys. Rev. Lett., 29, 81 (1972).

${ }^{50}$ C.M. Surko and R.E. Slusher, Phys. Rev. Lett., 36, 1747 (1976).

${ }^{51}$ K. Dzierzega, W. Zawadzki, B. Pokrzywka, and S. Pellerin, Phys. Rev. E, 74, 026404 (2006).

${ }^{52}$ A.L. Peratt, R.L. Watterson, and H. Derfler, Phys. Fluids, 20, 1900 (1977).

${ }^{53}$ F. Pisani, T. Pierre, and D. Batani, J. Plasma Phys., 59, 69 (1998).

${ }^{54}$ Y. Takase, J.D. Moody, C.L. Fiore, F.S. McDermott, M. Porkolab, and J. Squire, Phys. Rev. Lett., 59, 1201 (1987).

${ }^{55}$ G.A. Wurden, K.L. Wong, and M. Ono, Phys. Fluids, 28, 716 (1985).

${ }^{56}$ B.K. Sawhney, V.K. Tripathi, and S.V. Singh, Phys. Plasmas, 2, 760 (1995).

${ }^{57}$ D.L. Brower, W.A. Peebles, S.K. Kim, and N.C. Luhmann Jr., Rev. Sci. Instrum., 59, 1559 (1988).

${ }^{58}$ T.L. Rhodes, W.A. Peebles, X. Nguyen, M.A. VanZeeland, J.S. deGrassie, E.J. Doyle, G. Wang, and L. Zeng, Rev. Sci. Instrum., 77, 10E922 (2006).

${ }^{59}$ N.C. Luhmann Jr. and W.A. Peebles, Rev. Sci. Instrum., 55, 279 (1984).

${ }^{60}$ E. Holzhauer and G. Dodel, Rev. Sci. Instrum., 61, 2817 (1990).

${ }^{61}$ N.M. Kaganskaya, M. Kramer, and V.L. Selenin, Phys. Plasmas, 8, 4694 (2001).

${ }^{62}$ A.B. Altukhov, E.Z. Gusakov, M.A. Irzak, M. Kramer, B. Lorenz, and V.L. Selenin, Phys. Plasmas, 12, $022310(2005)$.

${ }^{63}$ J.G. Kwak, S.J. Wang, S.K. Kim, and S. Cho, Phys. Plasmas, 13, 074503 (2006).

${ }^{64}$ M. Kramer, Y.M. Aliev, A.B. Altukhov, A.D. Gurchenko, E.Z. Gusakov, and K. Niemi, Plasma Phys. Control. Fusion, 49, A167 (2007). 


\section{Chapter 2: Experimental Apparatus}

The WVU helicon plasma experiment is comprised of two distinct regions: (1) The Hot hELIcon eXperiment (HELIX) plasma production region and (2) the Large Experiment on Instabilities and Anisotropies (LEIA) expansion region. Plasma created in the HELIX source region flows into the larger LEIA chamber, which has a weaker magnetic field. The resultant higher beta $\left(\beta=n k_{B} T \mu_{0} / B^{2}\right)$ LEIA plasma is ideally suited for both magnetospherically and heliospherically relevant experimental studies. The geometry of the magnetic field expansion region between HELIX and LEIA also enables studies of spontaneous, current-free, electrostatic double layer formation at low neutral pressures.

In this chapter, the entire HELIX-LEIA experimental apparatus is described. However, all of the experiments described here were performed in the source region with the LEIA electromagnets turned off. Additional descriptions of the experimental apparatus may be found in Refs. $[1,2,3]$. A picture of the HELIX-LEIA plasma system with the $300 \mathrm{GHz}$ (mm-wave) based, collective Thomson scattering (CTS), apparatus in the foreground is shown in Figure 2.1. 


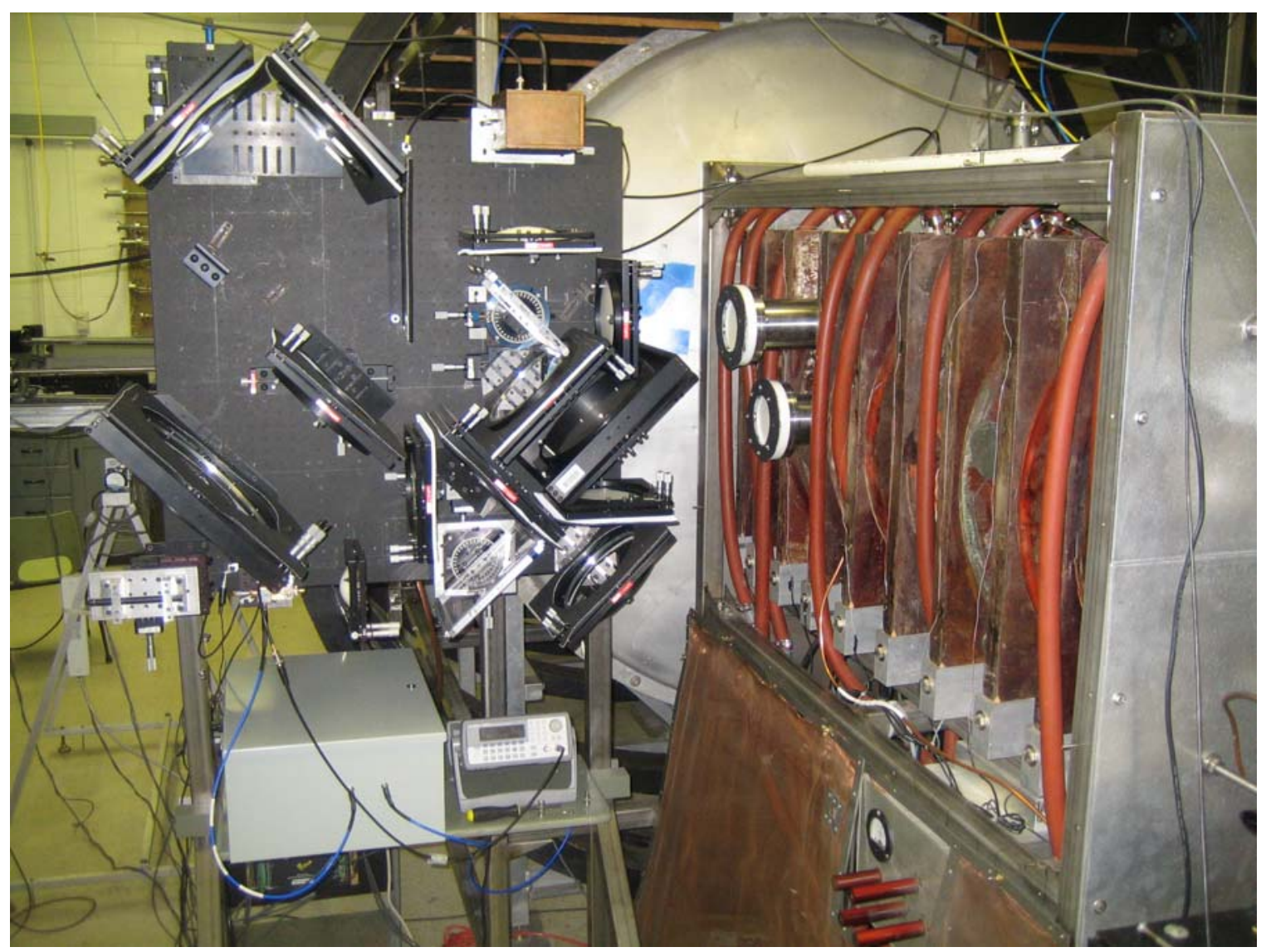

Figure 2.1 The HELIX source chamber region is the rectangular area in the foreground on the right and the LEIA space chamber is the large round silver chamber in the background. The $300 \mathrm{GHz}$ optical stand is in the foreground on the left.

\subsection{HELIX Chamber}

The HELIX vacuum chamber is a $61 \mathrm{~cm}$ long, Pyrex tube $10 \mathrm{~cm}$ in diameter connected to a $91 \mathrm{~cm}$ long, $15 \mathrm{~cm}$ diameter, stainless steel chamber. The chamber has one set of four 6" Conflat ${ }^{\mathrm{TM}}$ crossing ports in the center of the chamber and four sets of four 2 $3 / 4$ " Conflat ${ }^{\mathrm{TM}}$ crossing ports on either side that are used for diagnostic access. Two of the four 6" crossing ports, which were previously used for laser induced fluorescence (LIF) access, have been modified for CTS diagnostic access. The end of the stainless steel chamber is connected to LEIA, a $1.8 \mathrm{~m}$ diameter, $4.4 \mathrm{~m}$ long expansion chamber. The far end of the LEIA chamber, away from HELIX, is connected to a turbomolecular pumping 
station. The end of the HELIX chamber, opposite LEIA, is connected to a glass cross. The other three legs of the cross are terminated with another pumping station, an ion gauge, and a 12" stainless steel flange fitted with a 4 " viewport, respectively. A schematic of the combined HELIX and LEIA system is shown in Figure 2.2.

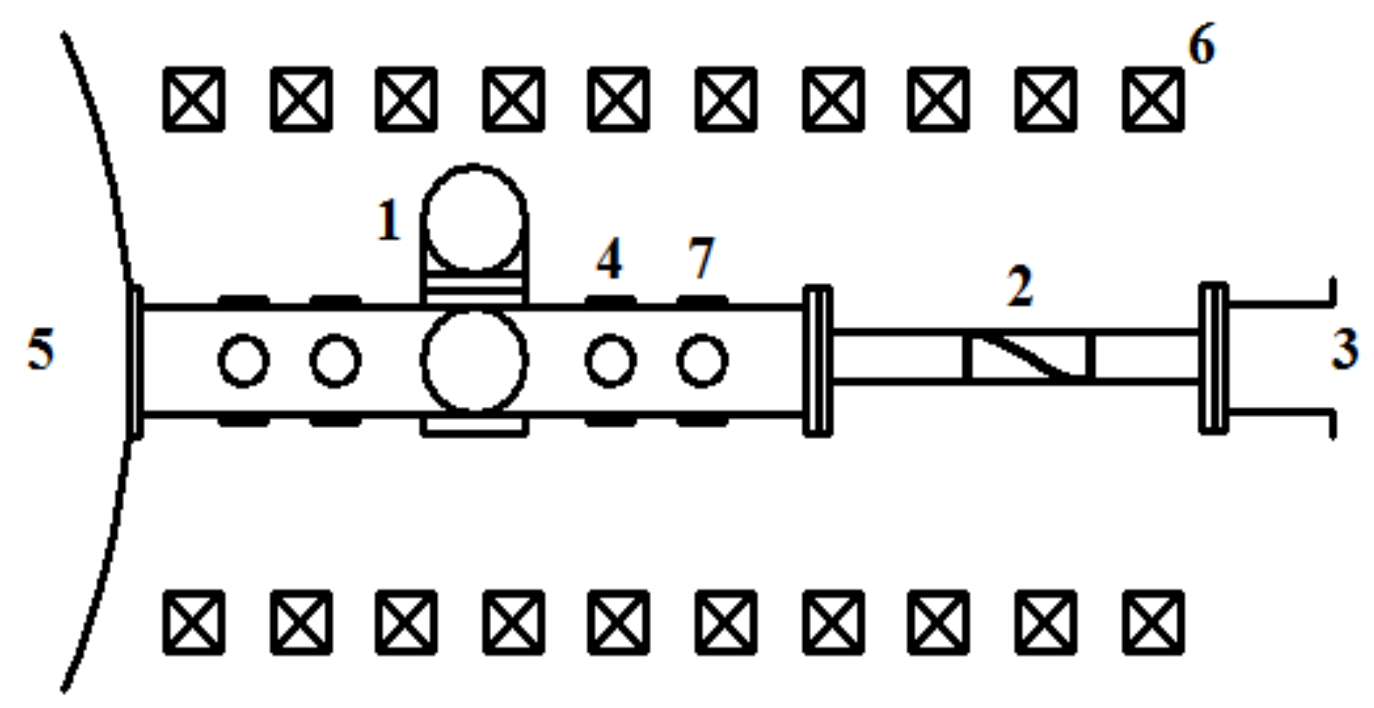

Figure 2.2 Schematic (side view) of the HELIX plasma source: (1) injection and collection ports for CTS, and probe flange (2) fractional helix antenna, (3) pumping station, gas inlet, and cold cathode pressure gauge (4) Baratron pressure gauge, and the additional gas inlet, (5) LEIA space chamber and pumps (6) HELIX magnetic field coils, and (7) retractable RF compensated Langmuir probe and fixed perpendicular LIF optics.

One significant change to the HELIX chamber from previous work is the addition of two 4" diameter tubes used for optical access of the $300 \mathrm{GHz}$ CTS system; at location 1 in Figure 2.2. The 4" tubes permit the CTS beams to pass between the magnetic field coils surrounding HELIX. A cut away of location 1 in Figure 2.2 (shown in Figure 2.3) shows both additional vacuum tubes: (1) A "T" shaped chamber above HELIX, and (2) a straight extension to the left HELIX. The "T" shaped chamber is 38 " long and extends beyond the magnetic field coils and the Faraday cage surrounding HELIX. Each end of the "T" has a 6" diameter Conflat ${ }^{\mathrm{TM}}$ flange to attach additional components. The center 
leg "T" has a 3" base that terminates in a 6" Conflat" flange for connection to the HELIX chamber. The straight extension to the left of HELIX is 15 " long, terminates in a 6" diameter Conflat ${ }^{\mathrm{TM}}$ at each end, and also extends beyond the magnetic field coils and Faraday cage. A detailed description of the components mounted on the chambers can be found in Chapter 6.

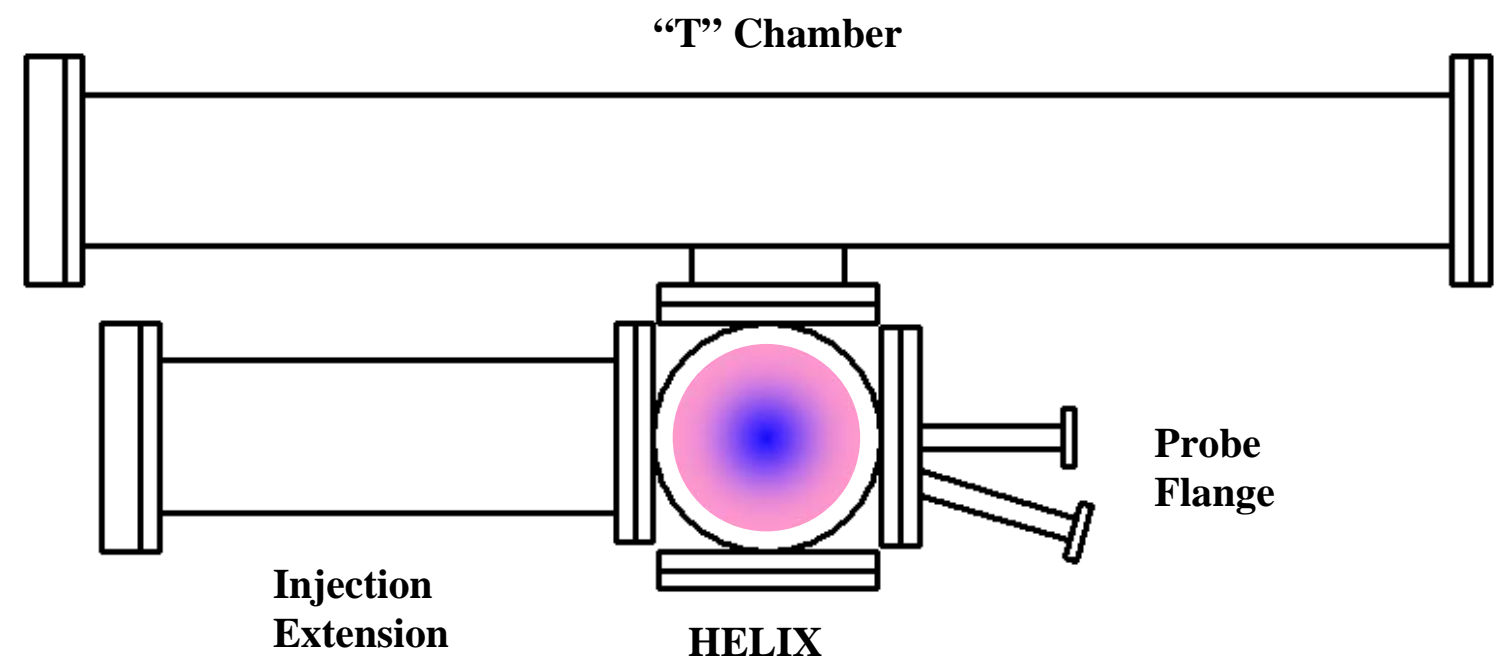

Figure 2.3 Cut away view of HELIX at the 6" crossing port with the new vacuum chamber extensions and probe flange.

Another recent addition to the HELIX chamber is the probe flange at the 6" crossing port (Figure 2.3). The flange is a modified 6" Conflat ${ }^{\mathrm{TM}}$ blank flange fitted with two stainless steel tubes and QF-40 flanges, separated by 18 degrees to allow both ports access to the center of HELIX. The flange was specifically designed for the wave launching antenna (discussed in Chapter 2.5) and the electrostatic double probe (discussed in Chapter 3.2). The angled port allows for positioning of the wave launching antenna in the path of the CTS diagnostic. The straight port is used for the electrostatic double probe, which measures fluctuations in the scattering volume of the CTS diagnostic beam line. 


\subsection{Vacuum System}

The vacuum in the chamber is maintained by a set of three turbomolecular drag pumps, each backed by a diaphragm roughing pump. A single Balzers TMU 520 turbomolecular drag pump is connected to one leg of the glass cross at the end of HELIX. A MDC GV-4000M-P 6 inch inner diameter gate valve is located between the turbo pump and the glass cross. Two Pfeiffer TMU 1600 turbomolecular pumps are connected to the far end of the LEIA chamber. Each turbo pump is backed with a diaphragm roughing pump to avoid contamination from oil based roughing pumps. Two MDC GV8000V-P 10 inch gate valves separate the turbo pumps from the LEIA chamber.

The three pumps maintain a base pressure on the order of $10^{-7}$ Torr. The pressure is measured by two Balzers PKR250 full range pressure gauges and a Baratron ${ }^{\circledR}$ capacitance manometer. The Balzers gauges achieve full range by combining a Pirani gauge for pressure above $10^{-2}$ Torr and a cold cathode gauge for pressures below $10^{-2}$ Torr. ${ }^{4}$ One advantage of the Baratron gauge is that the measurement is independent of gas species, while the Balzers gauges require correction depending on the gas species. The pressure gauge for the HELIX pumping station is located on one branch of the glass cross. The gauge for the LEIA pumping station is located on LEIA, toward the pumping station end of the chamber. Two MKS1179 mass flow valves, controlled by a PR-4000 power supply, regulate the gas flow to maintain the desired neutral pressure. The gasses used for the plasma discharges can be introduced at two locations in HELIX. One location is next to the Balzers pressure gauge on the glass cross (location 3 in Figure 2.2). In previous experiments, this "end" gas feed location was typically used. A new location for gas introduction is at one of the $23 / 4$ " crossing ports (location 4 in Figure 2.2) on the 
stainless steel portion of the chamber. The second gas feed location allows for a more direct gas flow into the plasma chamber near the antenna instead of relying on diffusion overcoming the pumping at the "end" feed location. The Baratron ${ }^{\circledR}$ capacitance manometer pressure gauge, located at position 4 in Figure 2.2, is used for an absolute calibration of the Balzers gauges. Typical neutral operating pressures in HELIX range from 0.1 to 100 mTorr.

After the addition of the new center feed gas inlet at location 4 of Figure 2.2, a recalibration of the HELIX and LEIA chamber pressure readings was performed. Pressure readings were obtained with both Balzers pressure gauges as well as the Baratron capacitance manometer for argon and helium gas; with the gas fed at either the end of HELIX or the new gas inlet; and with the gate valve at the end of HELIX open or closed. Opening and closing the HELIX gate valve provides additional control of the neutral gas pressure. The calibration was performed without a plasma discharge since the rf noise and match quality can alter the readings of the gauges and the objective was to determine the neutral pressure gradient along the axis of the source. Although the Balzers gauge measurements do require correction due to the gas species, for the scope of this discussion, only the neutral pressures as measured by the Baratron for argon are shown in Figure 2.4. See Appendix A for all data and graphs related to determining the pressure gradient along the axis of the entire system, including data for helium and the Balzers gauge pressures in LEIA. 

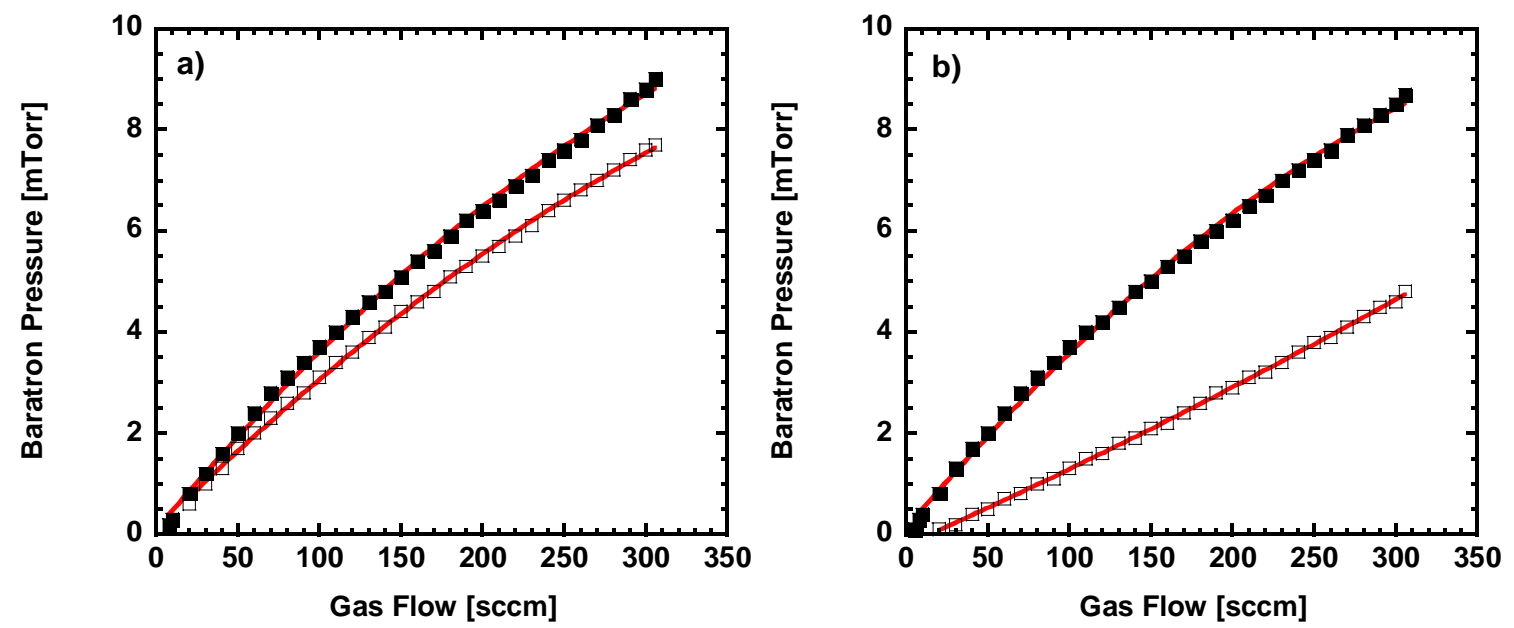

Figure 2.4 Baratron gauge pressure as a function of gas flow for argon in HELIX. (a) center feed and (b) end feed. The data points are (ם) HELIX gate valve closed (ם) HELIX gate valve open.

Fits to the data in Figure 2.4 are used to determine the neutral gas pressure in the center of HELIX as a function of the gas flow, with the gas fed at either the end of HELIX or the new gas inlet, and with the gate valve at the end of HELIX open or closed:

$$
\begin{aligned}
& P_{B}=0.094+0.038 F_{G}-3.22 \times 10^{-5} F_{G}^{2} \\
& P_{B}=0.113+0.031 F_{G}-2.35 \times 10^{-5} F_{G}^{2} \\
& P_{B}=0.131+0.038 F_{G}-3.43 \times 10^{-5} F_{G}^{2} \\
& P_{B}=-0.207+0.014 F_{G}-5.97 \times 10^{-6} F_{G}^{2}
\end{aligned}
$$

where $P_{B}$ is the pressure as measured by the Baratron gauge and $F_{G}$ is the gas flow rate. Equations 2.1 and 2.2 are fits to the data of Figure 2.4a, while Equations 2.3 and 2.4 are fits to the data in Figure 2.4b. When the gate valve is open for the center feed (Figure 2.4a), the pressure in the center of HELIX is reduced by approximately 1.5 mTorr at the largest flow rates as compared to when the gate valve is closed. For the end feed, there is a significant difference in the pressure at the center of HELIX for all flow rates when the gate valve is open (Figure 2.4b). To achieve the largest range of neutral pressures with minimal system adjustment, all the experiments described in this work used the new gas 
feed in the center of HELIX.

\subsection{Magnetic Field Generation}

Ten electromagnets produce a steady state axial magnetic field of 0-1300 Gauss in HELIX. Each magnet has 46 internal copper windings with a resistance of $17 \mathrm{~m} \Omega$ and an inductance of $1.2 \mathrm{mH}$. The magnets are water-cooled and their axial positions are adjustable along a set of rails. Two Xantrex 200 Amp power supplies operated in parallel provide the current to the electromagnets. The Xantrex power supplies replace the original MacroAmp power supply which was found to have significant current fluctuations when not operated at the maximum output current.

The LEIA magnetic field is produced by a set of seven custom-built, 9' diameter electromagnets. Each magnet consists of five sets of aluminum tubing wound into five two-coil "pancakes" of four layers each, for a total of 40 turns per magnet. The 0.5 " $\mathrm{x}$ 0.5 " square tubing is hollow and wrapped in an insulating paper. The magnets are watercooled by a closed loop system maintained with a Neslab HX-300 chiller. These magnets are upgraded versions of the original 20 turn coils used previously on LEIA. ${ }^{1,3}$ In these new electromagnets, the larger size aluminum tubing (and therefore lower electrical resistance) and increased number of turns provide a factor of two increase in the magnetic field strength for the same total input power as in the original electromagnets. Additionally, the inner hole of the new tubing is circular, rather than rectangular; allowing for better attachment of the water connections. A LEIA magnetic field ranging from 0-130 Gauss is created using a 200 Amp DC EMHP power supply.

Upon completion of the LEIA electromagnet coil construction and installation of the new HELIX power supplies, measurements of the axial $(r=0)$ magnetic field strength 
were performed (shown in Figures $2.5-2.6){ }^{2}$ Note that although the experiments in this work do not include any magnetic fields in LEIA, the magnetic field near the HELIXLEIA junction rises to a peak before decreasing to essentially a constant magnitude through LEIA. Without the LEIA magnets on (not shown) the magnetic field strength is essentially constant throughout HELIX.

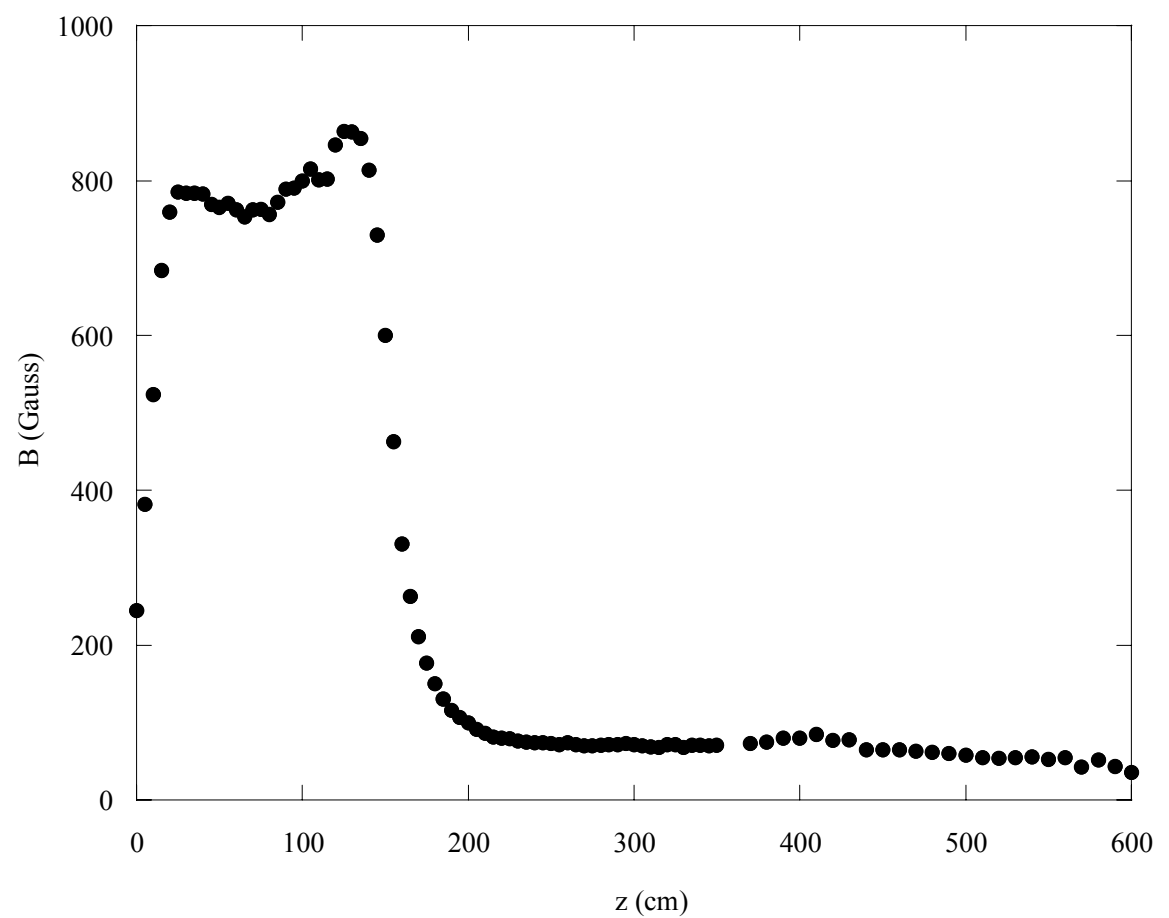

Figure 2.5 Axial profile of the magnetic field at $r=0$ for a current of 220 Amps in the HELIX magnets and 100 Amps in the LEIA magnets. The axial distance is measured from the end of the HELIX Pyrex chamber and increases towards LEIA. Figure obtained from Ref. [2]. 


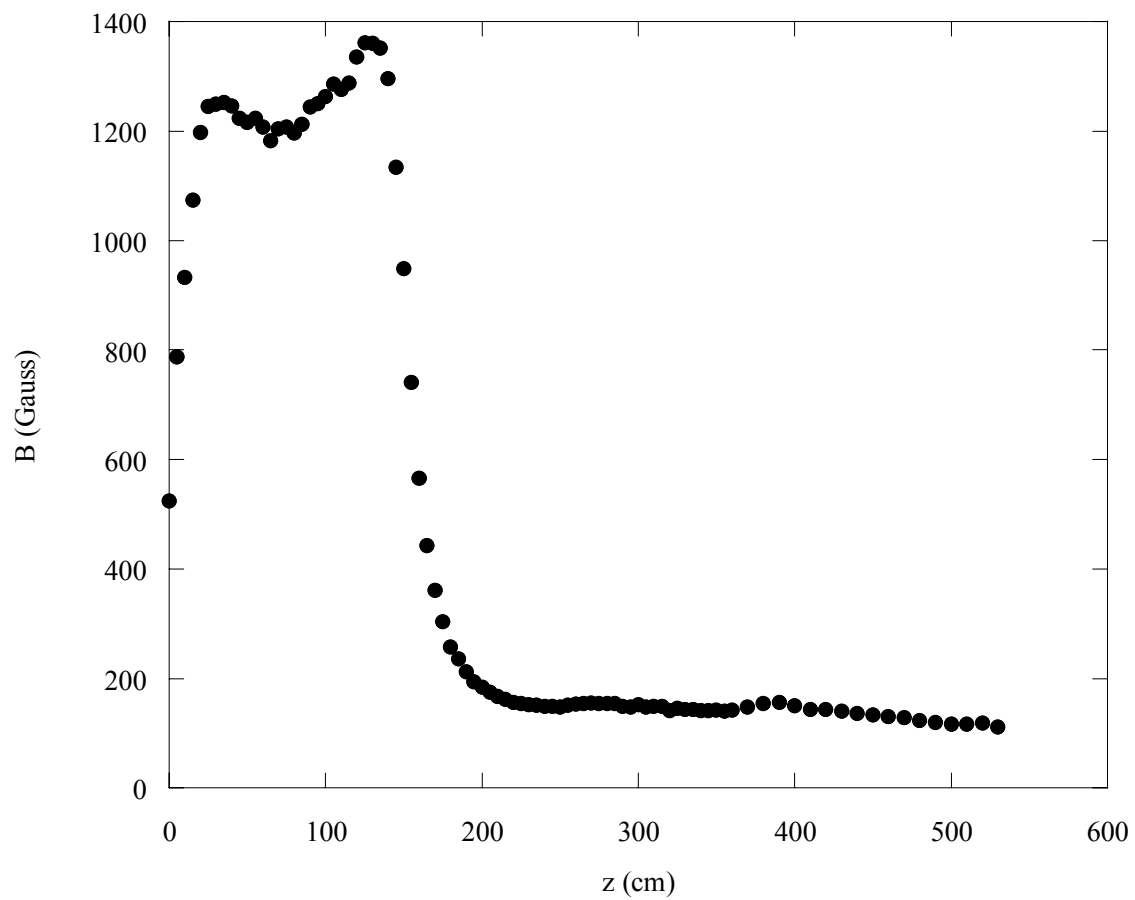

Figure 2.6 Axial profile of the magnetic field at $r=0$ for a current of 345 Amps in the HELIX magnets and 200 Amps in the LEIA magnets. The axial distance is measured from the end of the HELIX Pyrex chamber and increases towards LEIA. Figure obtained from Ref. [2].

\subsection{RF Antenna and Matching Network}

For the experiments reported in this work, the plasma was created in HELIX with a $19 \mathrm{~cm}$ half wave, right-handed helix antenna wrapped around the outside of the Pyrex vacuum chamber. Rf power is supplied to the antenna from an ENI $200030 \mathrm{~dB}$ amplifier providing up to $2 \mathrm{~kW}$ of power over the frequency range 6-18 MHz. The initial $\mathrm{rf}$ wave is provided by a $50 \mathrm{MHz}$ Wavetek function generator. The rf power is coupled to the antenna through a $\pi-$ matching network that matches the inductive load of the antenna to the amplifier's output impedance of 50 Ohms. ${ }^{3}$ The matching network contains one load capacitor and three tuning capacitors, all of which are Jennings high voltage tunable vacuum capacitors. The load capacitor has a tunable range of 20-2000 pF, two of the tuning capacitors have a range of $4-250 \mathrm{pF}$, and the third tuning capacitor has a range of 5-500 pF. The capacitors are connected by sheets of copper, which are connected to the 
antenna by rods of silver-plated copper. A schematic of the matching circuit and connections to the antenna is shown in Figure 2.7.
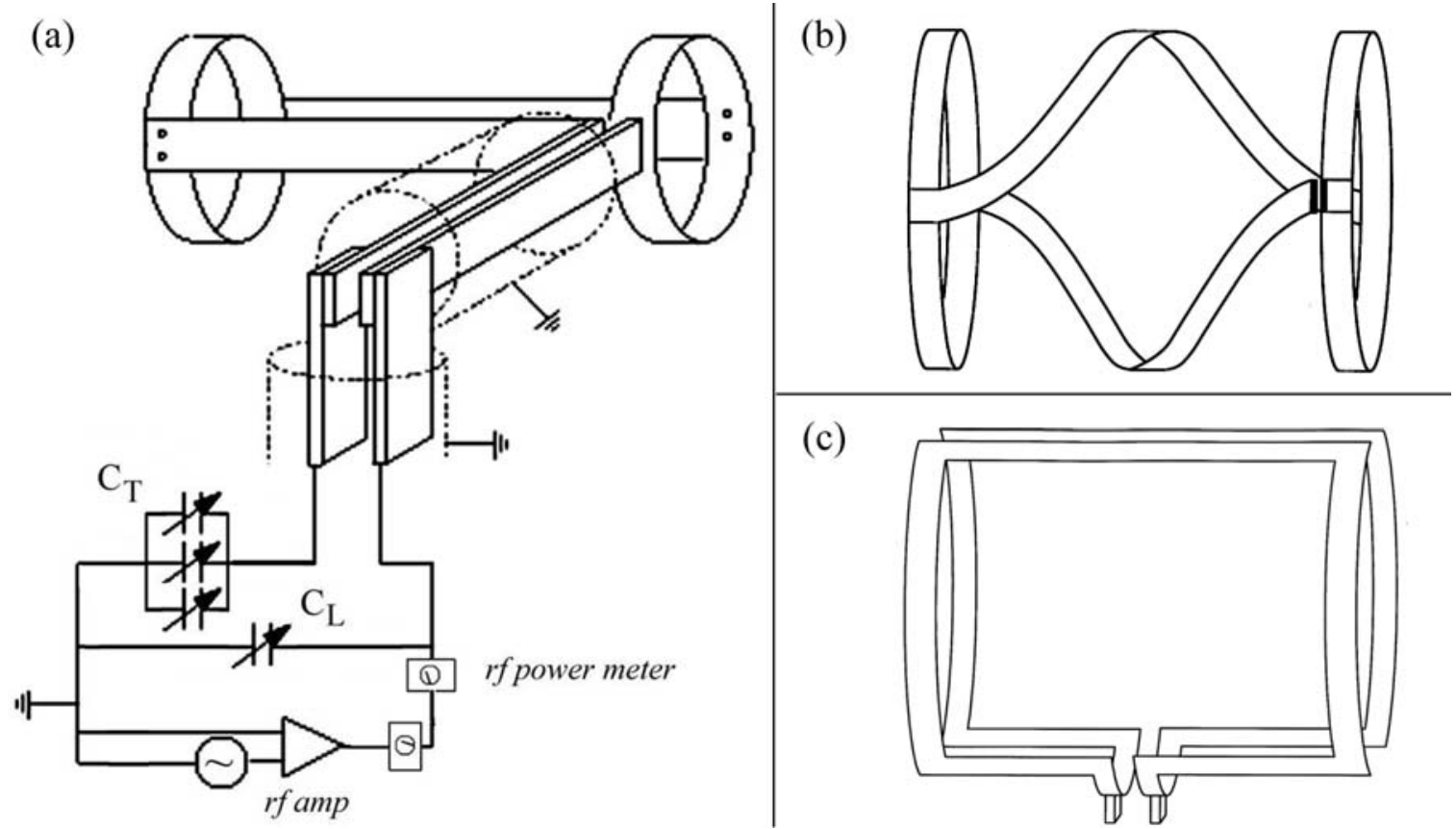

Figure 2.7 a) Antenna matching circuit for HELIX. $\mathrm{C}_{\mathrm{T}}$ are the tuning capacitors and $\mathrm{C}_{\mathrm{L}}$ is the load capacitor. b) and c) show two additional RF antenna designs. Antenna b) is used in this work. Figure obtained from Ref. [2].

\subsection{Electrostatic Wave Launching Antenna}

Because the objective of this project is the detection of spontaneously excited short wavelength fluctuations at a specific set of operating parameters, it seemed prudent to construct an antenna capable of artificially exciting electrostatic waves that could be detected with the $300 \mathrm{GHz}$ CTS diagnostic. A schematic of the antenna apparatus is shown in Figure 2.8. The emitter head is a 0.5 " diameter, 0.035 " thick, stainless steel mesh that is spot welded to the end of a slotted 0.125 " diameter copper rod (See Figure 2.9). The copper rod is surrounded by a $0.188^{\prime \prime}$ outer diameter (OD) alumina tube, a 0.219 " OD stainless steel tube, a 0.375 " OD alumina tube, and finally a 0.5 " OD stainless steel tube. The alumina tubing electrically isolates the individual stainless steel tubing 
sections. The stainless steel tubing serves as the vacuum seal as well as electromagnetically shielding the central copper rod. At the end of the copper rod, opposite the emitter head, a bulkhead BNC connector center pin is soldered into a cavity cut into the copper rod. The BNC shield provides the ground connection to the stainless steel tubing. An RG-58 cable with the outer insulation removed couples the antenna signal from the BNC bulkhead mount to a BNC vacuum feedthrough inside a $23 / 4$ " CF nipple. Since the cable is in vacuum the BNC cable is stripped down to remove the exterior covering.

(a)

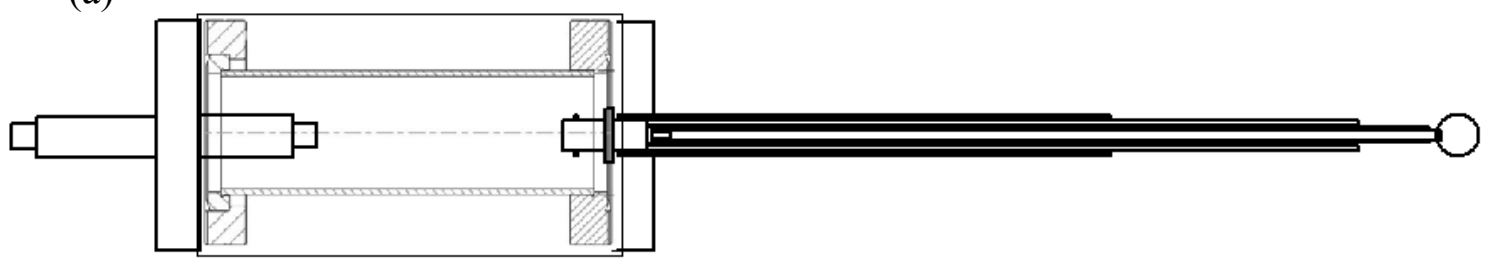

(b)

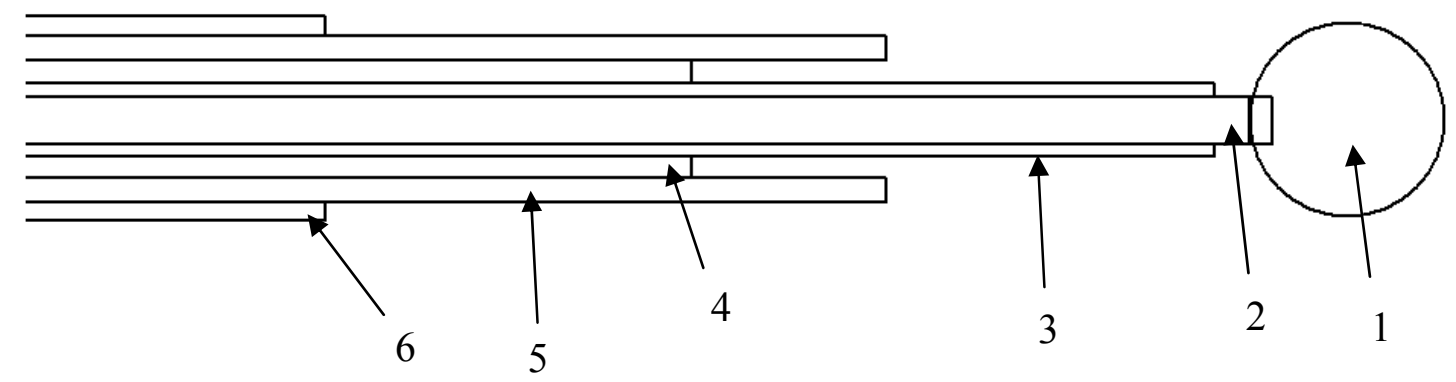

Figure 2.8 a) Electrostatic wave launching antenna assembly. b) Enlarged view of the antenna including 1) emitter head, 2) copper rod, 3) alumina tube, 4) stainless steel tube, 5) alumina tube, and 6) stainless steel tube.

The mesh antenna is driven by an RF Power Labs, model FK 30-50, 50 Watt linear power amplifier with an input voltage limited to $1 \mathrm{~V}$ peak-to-peak. The input to the amplifier is supplied by a Hewlett-Packard, model 33120A, $15 \mathrm{MHz}$ function generator. 
The exact frequency range of the amplifier is unknown since no manual is available, but the amplifier appeared to function over the frequency range $150 \mathrm{kHz}$ to at least $5 \mathrm{MHz}$.

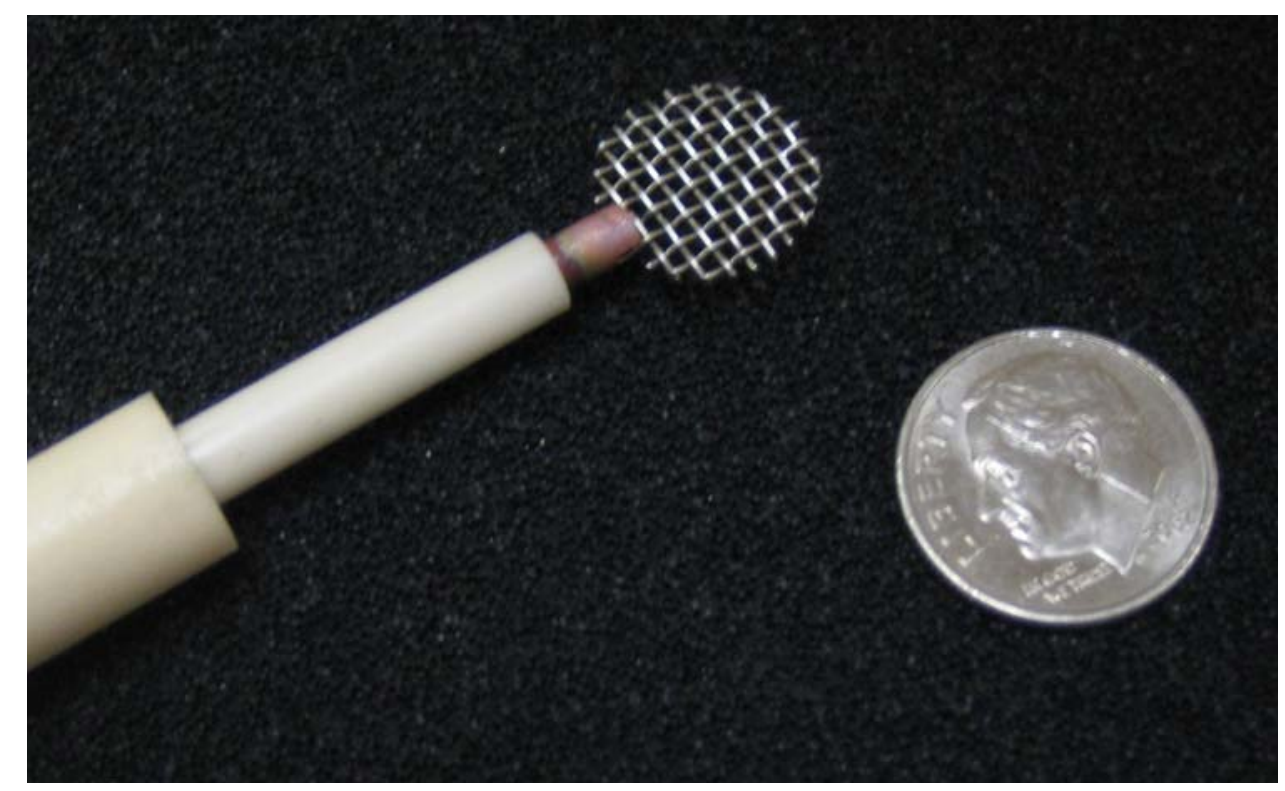

Figure 2.9 The completed electrostatic wave launching antenna.

\subsection{Plasma Parameters}

The HELIX plasma source can be operated in either a pulsed or steady state mode and over a wide range of plasma parameters. For these experiments argon plasmas were produced in the steady state at varying rf frequencies and HELIX magnetic field strengths while the neutral filling pressure $(8 \mathrm{mTorr})$ and RF power $(500 \mathrm{~W})$ were kept constant. The LEIA magnetic field was kept at zero unless otherwise noted. Typical HELIX operating parameters are listed in Table 2.1. 
Table 2.1 Typical plasma parameters in HELIX.

\begin{tabular}{|l|c|}
\hline \multicolumn{1}{|c|}{ Plasma Parameter } & Typical Value in HELIX \\
\hline Gas Species & argon, helium, nitrogen, xenon \\
\hline Base Pressure & $<2 \times 10^{-7}$ Torr \\
\hline Operating Pressure & 0.1 to 100 mTorr \\
\hline Magnetic Field & $<1200$ Gauss \\
\hline RF Power & 0 to $2 \mathrm{~kW}$ \\
\hline Operating Frequency & $6-18 \mathrm{MHz}$ \\
\hline Density & $\leq 3 \times 10^{13} \mathrm{~cm}^{-3}$ \\
\hline Electron Temperature & $\sim 5 \mathrm{eV}$ \\
\hline Ion Temperature & $\leq 1 \mathrm{eV}$ \\
\hline Electron Gyro-Radius & $\sim 0.04 \mathrm{~mm}$ \\
\hline Ion Gyro-Radius & $\sim 2.7 \mathrm{~mm}$ \\
\hline
\end{tabular}




\section{Chapter 2 References}

${ }^{1}$ J.L. Kline, Slow Wave Ion Heating and Parametric Instabilities in the HELIX Helicon Source, Ph.D. Dissertation, West Virginia University, Morgantown (2002).

${ }^{2}$ A.M. Keesee, Neutral Density Profiles in Argon Helicon Plasmas, Ph.D. Dissertation, West Virginia University, Morgantown (2006).

${ }^{3}$ M.M. Balkey, Optimization of a Helicon Plasma Source for Maximum Density with Minimal Ion Heating, Ph.D. Dissertation, West Virginia University, Morgantown (2000).

${ }^{4}$ Balzers, Operating Manual for PKR 250 Compact Full Range Gauge, Balzers Aktiengesellschaft, FL9496 Balzers, Fürstentum Liechtenstein. 


\section{Chapter 3: Standard Diagnostics}

The standard suite of diagnostics used to measure HELIX plasma parameters is described in this chapter. The details of the $300 \mathrm{GHz}$ CTS diagnostic system are provided in Chapter 6.

\subsection{Measurement of Plasma Density and Electron Temperature}

Arguably one of the oldest and most widely used diagnostic, particularly in low temperature plasmas, is the Langmuir probe. In its simplest form, the Langmuir probe consists of a single conductor placed into plasma to obtain local parameters such as electron density and temperature. Measurements, in general, are performed by applying a bias to the conductor with a voltage and measuring the current drawn. Albeit a simple measurement to perform, the analysis of a Langmuir probe measurement is non-trivial. One typical assumption for simplifying the analysis is that the plasma is a stationary Maxwellian. One drawback to consider with the Langmuir probe, as with any probe physically introduced in the plasma, is the perturbative effects that probes have on the plasma. There are several reviews available in the literature for both the theory and operation of Langmuir probes such as Hutchinson, ${ }^{1}$ Schott, ${ }^{2}$ Chen, ${ }^{3}$ Hershkowitz, ${ }^{4}$ and Demidov $^{5}$; only highlights of the principle aspects are presented here.

\subsubsection{Langmuir Probe Theory}

A classic Langmuir probe measurement consists of applying a bias voltage to a conductor immersed in the plasma and measuring the current drawn. By sweeping the probe through a range of bias voltages and measuring the corresponding currents, the 
relationship commonly known as an I-V characteristic is obtained. An example of an ideal I-V characteristic is shown in Figure 3.1.

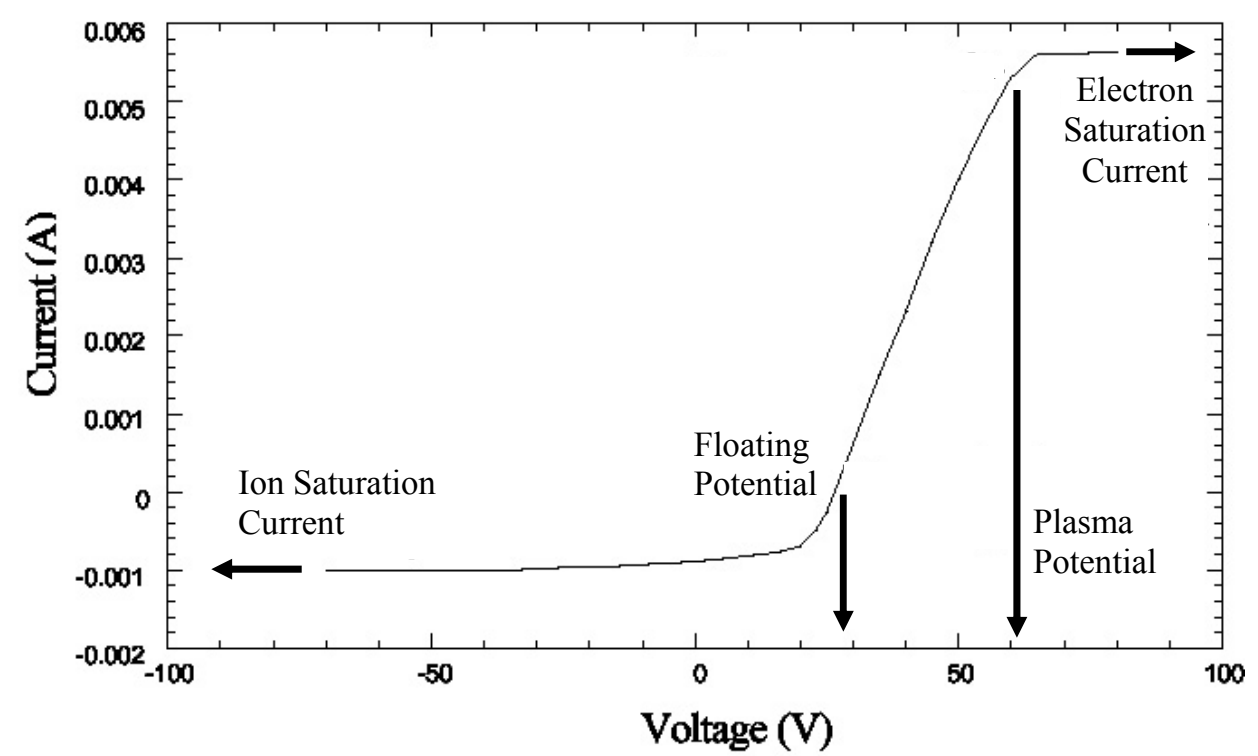

Figure 3.1 Idealized Langmuir probe I-V characteristic. Figure obtained and modified from Ref. [6].

If a Langmuir probe were placed in the plasma without a bias or connection to electrical ground, the probe would charge negatively until reaching a potential at which the current to the probe vanished. The potential at which the current vanishes is called the floating potential, i.e., the potential at which the electron and ion fluxes to the probe are equal. The reason that the probe charges negatively is, because of their lighter mass and a larger mean velocity, electrons have a larger flux to the probe than ions.

Variation of the probe's bias voltage is accomplished by connecting the probe to a voltage source. When the applied voltage is more negative that the floating potential, the probe attracts ions and repulses the electrons, resulting in a net positive current. As the negative bias is increased, a collection limit of the ion current will be reached; the ion saturation current. A similar process occurs when the bias voltage is more positive than the floating potential. For a bias voltage more positive than the floating potential, the 
probe attracts more electrons while repulsing the ions. The voltage at which there is no potential difference between the probe and the plasma, and the collected current begins to saturate is the plasma potential. At a large enough positive bias, the collection limit of the electron current is reached and this is the electron saturation current. The magnitude of the electron saturation current is larger than the ion saturation current because the electrons are more mobile. Both the ion and electron saturation currents are dependant on the probe size, plasma density, and electron temperature.

To determine the plasma parameters from an I-V characteristic, first recall the assumptions that the plasma constituents obey a Maxwellian velocity distribution, are non-drifting and collisionless. With these assumptions, the total electrical current, $I\left(V_{0}-V_{p}\right)$, collected by the probe is

$$
I\left(V_{0}-V_{p}\right)=n_{e} e A_{p}\left(\frac{T_{e}}{m_{i}}\right)^{1 / 2}\left[\frac{1}{2}\left(\frac{2 m_{i}}{\pi m_{e}}\right)^{1 / 2} \exp \left(\frac{e\left(V_{0}-V_{p}\right)}{T_{e}}\right)-\frac{A_{s}}{A_{p}} \exp \left(-\frac{1}{2}\right)\right],
$$

where $n_{e}$ is the plasma density, $e$ is the charge, $A_{p}$ is the surface area of the probe, $T_{e}$ is the electron temperature, $m_{e}$ and $m_{i}$ are the electron and ion mass respectively, $V_{0}$ is the applied voltage, $V_{p}$ is the plasma potential, and $A_{s}$ is the area of the sheath surface. ${ }^{1}$ The voltage seen by the plasma is the difference between the applied voltage and the plasma potential, $V \equiv V_{0}-V_{p}$. The second term inside the bracket of Equation 3.1 is the ion saturation current, $I_{s i}$, in an unmagnetized plasma

$$
I_{s i}=-e J_{i}=-0.61 e n_{e} A_{p} \sqrt{T_{e} / m_{i}} .
$$

There are two unknowns in Equation 3.1, the plasma density and the electron temperature. The electron temperature, is obtained from the derivative of Equation 3.1 with respect to $V$, 


$$
\frac{d I(V)}{d V} \cong \frac{e}{T_{e}}\left(I-I_{s i}\right)+\frac{d I_{s i}}{d V} .
$$

By examination of Figure 3.1, we see that $d I_{s i} / d V \ll d I(V) / d V$. Therefore Equation 3.3 can be rewritten to provide an estimate of the electron temperature

$$
T_{e}=\frac{e\left[I(V)-I_{s i}\right]}{\frac{d I(V)}{d V}} .
$$

Experimentally, the electron temperature is calculated by performing a linear fit to $\ln \left|I-I_{s i}\right|$ versus $V$ and the electron temperature is the inverse of the slope of the fit. Using the calculated temperature and measured ion saturation current, the electron density is determined via Equation 3.2.

Cylindrical Langmuir probes cannot be driven into electron saturation in high density plasmas like the helicon source because of the sheath surrounding the probe continues to expand and electron saturation is never achieved. ${ }^{1}$ Since the Langmuir probe does not reach electron saturation, the plasma potential at the "knee" is not directly measured and has to be approximated.

For $T_{e}>T_{i}$, the floating potential is related to the plasma potential through a simple relationship. Given that the ion current at the floating potential is

$$
j_{i}=0.25 n e \sqrt{\frac{8 k T_{i}}{\pi m_{i}}}
$$

while the equal electron current at the floating potential is

$$
j_{e}=0.25 n_{e} e \sqrt{\frac{8 k_{b} T_{e}}{\pi m_{e}}} \exp \left(\frac{e\left(V_{f}-V_{p}\right)}{k_{b} T_{e}}\right) \text {, }
$$


where $k_{b}$ is Boltzmann's constant. ${ }^{1}$ Since at the floating potential the probe current is zero (and with some rearranging of terms),

$$
V_{p}=V_{f}+\frac{k_{b} T_{e}}{2 e} \ln \left(\frac{T_{e} m_{i}}{T_{i} m_{e}}\right) .
$$

Under the constraint $T_{e}>T_{i}, T_{i}$ can be replaced by $T_{e}$ in Equation $3.7^{1}$ and for argon ions, ( $m_{i}=40 m_{p}$, where $m_{p}$ is the mass of a proton), Equation 3.7 becomes

$$
V_{p} \approx V_{f}+5.6 T_{e} .
$$

Thus, in argon, the difference between the plasma and floating potentials is approximately six times the electron temperature.

One major aspect of Langmuir probe theory that has been ignored thus far, is the effect of a magnetic field. Since the ions and electrons gyrate around the magnetic field lines and cross field transport is limited, the flux of particles to the probe is restricted. The total effect depends on the on the ratio of the gyro-radius of each species to the size of the probe. Electrons have a smaller gyro-radius than the ions, and therefore the electron flux to the probe is preferentially reduced. However, since we do not bias our probe into electron saturation for density measurements, we need only consider the effect on the ions.

Magnetic field effects on the ions do necessitate a slight modification to Equation 3.2, since the magnetic field reduces the number of ions reaching the probe. In HELIX, for a magnetic field of $1000 \mathrm{G}$ and an ion temperature of $0.3 \mathrm{eV}$, the ion gyro-radius is approximately $3.5 \mathrm{~mm}$, which is of the same order of magnitude as the probe tip length (2 $\mathrm{mm}){ }^{1,7}$ Including the magnetic field effects, Equation 3.2 becomes

$$
I_{s i}=-0.49 e n_{e} A_{p} \sqrt{T_{e} / m_{i}} \cdot
$$


Another important effect on Langmuir probe measurement arises from rf fields in the helicon source. The rf fields constantly accelerate and decelerate the electrons toward the probe when the probe is near the floating potential, resulting in an error in the floating potential measurement and an apparent broadening of the electron velocity distribution function. ${ }^{8}$ A method, developed by Sudit and Chen, is used to compensate for the rf fields in rf driven plasmas. ${ }^{9}$ The addition of a floating electrode that is exposed to the plasma potential fluctuations and connected to the probe tip through a small capacitor forces the probe tip to follow the potential oscillations, thereby reducing the sheath impedance. The Langmuir probe used in HELIX has such a floating electrode, but is not directly exposed to the plasma. A set of $\mathrm{rf}$ chokes are also connected inline from the probe tip and the voltage source, increasing the impedance of the circuit at the rf frequency.

\subsubsection{Langmuir Probe Apparatus}

A schematic drawing of the Langmuir probe used in these experiments is shown in Figure 3.2. The probe tip is a $0.5 \mathrm{~mm}$ diameter graphite rod (mechanical pencil material) inserted into a $0.6 \mathrm{~mm}$ inner diameter alumina shaft and attached by a set screw to a copper base. A $10 \mathrm{nF}$ capacitor is also connected to the copper base. This assembly is placed inside a boron nitride $(\mathrm{BN})$ cap such that the probe tip extends into the plasma through a hole in the BN cap, while the opposite leg of the capacitor remains within the $\mathrm{BN}$ head so that it is not directly exposed to the plasma. The threaded BN cap attaches to the stainless steel probe shaft. A chain of RF chokes is attached to the copper base. The

RF chokes are $1 / 4$ Watt shielded inductors from Lenox-Fugle International, Inc. ${ }^{10}$, each especially designed to block a particular RF frequency. 


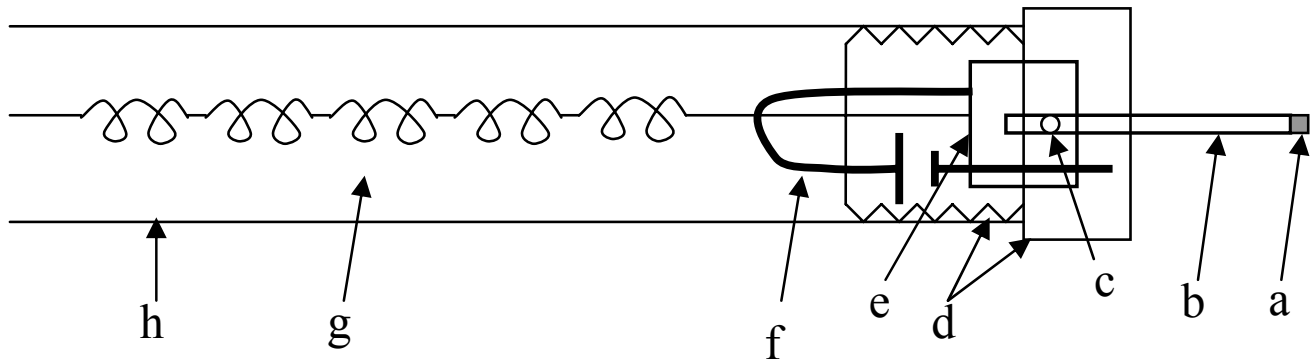

Figure 3.2 Schematic drawing of Langmuir probe design including a) graphite probe tip, b) alumina shaft, c) set screw, d) threaded boron nitride cap e) copper base, f) capacitor, g) chain of RF chokes, and h) stainless steel probe shaft. Figure obtained from Ref. [11].

Starting from the copper base, the rf chokes are placed in the order: $26,53,26,13.2$, and 6.8 MHz. The end of the rf choke string is then soldered to a shielded, coaxial connected probe wire that is attached to the $\mathrm{BNC}$ vacuum feedthrough at the far end of the probe shaft. Thermaflex tubing is used to cover the chain of rf chokes. Figure 3.3 is a picture of the Langmuir probe head used in HELIX. Note that the majority of the probe tip is $<1$ $\mathrm{mm}$ in diameter and thus plasma perturbation is minimized.

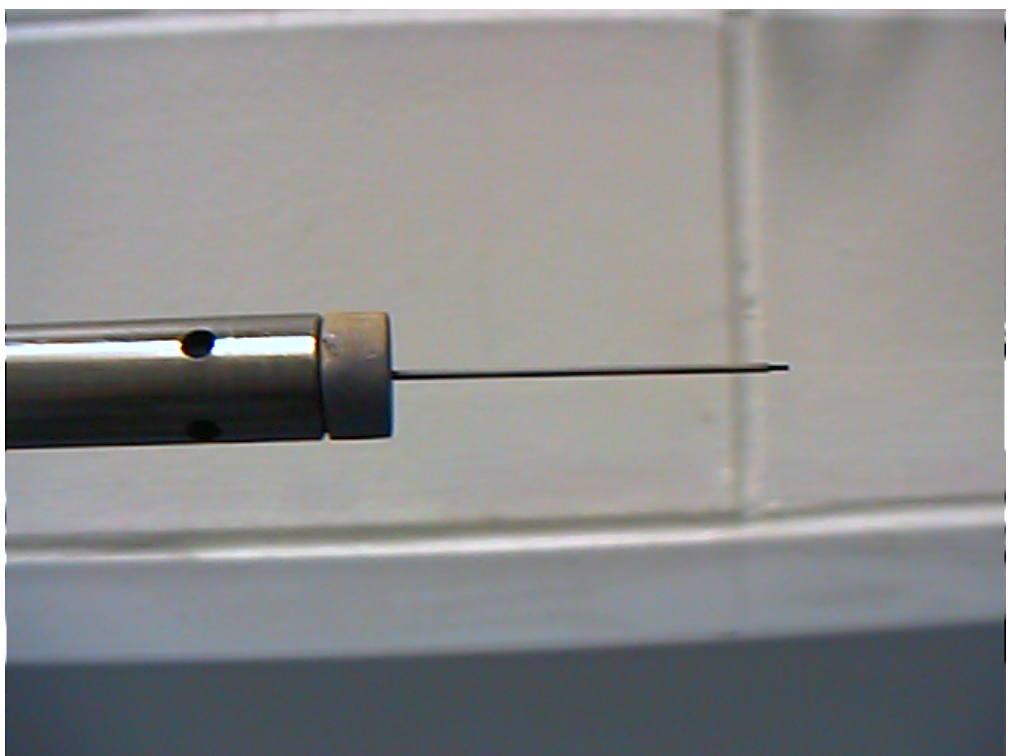

Figure 3.3 The Langmuir probe head. The exposed graphite tip is $2 \mathrm{~mm}$ long and runs the length of the alumina tube into the boron-nitride cap. Figure obtained from Ref. [12]. 
A Keithley 2400 SourceMeter is used to measure the Langmuir probe I-V trace. The source meter applies the bias voltage to the probe, as well as measures the collected

current. The source meter is controlled by custom software created with LabWindows ${ }^{\mathrm{TM}}$ via a GPIB interface. The Langmuir probe measurements used in these experiments were obtained $50 \mathrm{~cm}$ downstream of the antenna at position 7 in Figure 2.2.

\subsection{Electrostatic Fluctuation Measurement}

\subsubsection{Electrostatic Probe}

The electrostatic probe used to measure electrostatic fluctuations is essentially a two tip Langmuir probe. The two tips are separated spatially to measure differences in the floating potential as a function of time. The electrostatic probe has no $\mathrm{rf}$ compensation since the objective is to measure fluctuations in the floating potential at frequencies up to the rf driving frequency. We assume that the fluctuations are that of the floating potential and do not result from fluctuations in the electron temperature. The emissive probes ${ }^{13}$ typically used to determine electron temperature fluctuations do not work well in steadystate helicon plasmas with plasma densities on the order of $10^{13} \mathrm{~cm}^{-3}$.

The electrostatic probe used in this work, (Figure 3.4), consists of two tungsten tips, $2 \mathrm{~mm}$ long and $0.33 \mathrm{~mm}$ in diameter. The tips are separated by a center-to-center distance of $0.61_{-0.13}^{+0.33} \mathrm{~mm}$. The upper limit includes the center-to-center separation distance plus the individual radius of each tip while the lower limit is based on the resolution limit of the method used to measure the tip spacing. The tips protrude from a 6-bore alumina shaft, $2 \mathrm{~mm}$ in outer diameter, and extending $7.6 \mathrm{~cm}$ from a boron nitride (BN) cap. Just beyond the rear of the $\mathrm{BN}$ cap, the tungsten tips are soldered to the signal 
wires. The thermoflex covered signal wires are then fed through a 4-bore alumina shaft to keep the wires separated and insulated from each other. The signal wires then connect to a vacuum $\mathrm{BNC}$ feedthrough. The signal from each tip is connected to the Joerger VTR10012, a 12-bit resolution digitizer, with RG223U double shielded cables. The data records are transferred from the digitizer via a MIXI bus to a PC for later analysis. The possible orientations of the probe are shown in Figure 3.5. For a maximum measured phase difference of $\pi$ and a tip separation of $0.061 \mathrm{~cm}$, the maximum measureable wave number is $\pm 51.5 \mathrm{rad} / \mathrm{cm}$.

a)

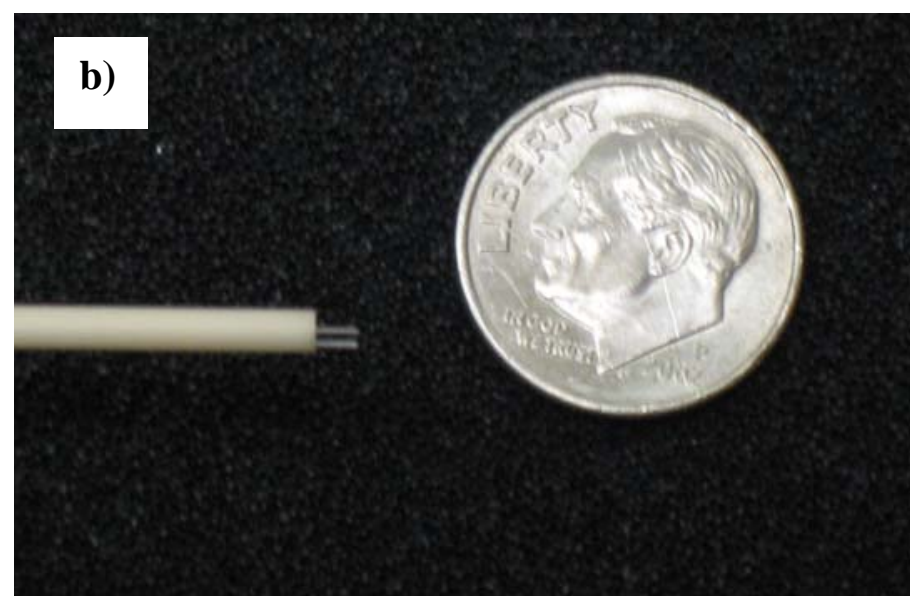

Figure 3.4 (a) End view of the electrostatic double probe. The hashed regions depict the locations of the tungsten tips in the alumina. (b) The electrostatic double probe.

a)

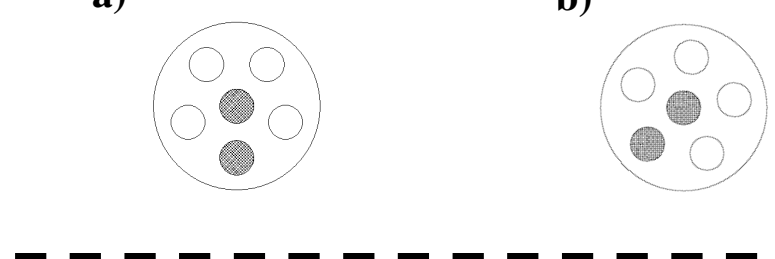

c)

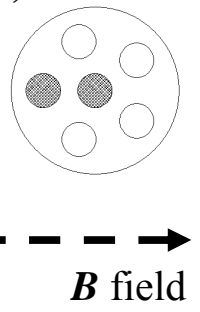

d)

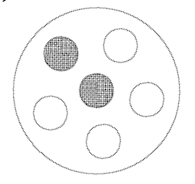

e)

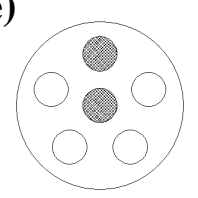

direction

Figure 3.5 Possible double probe orientation directions relative to the magnetic field direection: (a) perpendicular, (b) +45 degrees, (c) parallel, (d) -45degrees (e) anti-perpendicular. 


\subsubsection{Electrostatic Probe Analysis}

Measurement of the wave number of a wave, at an instant in time, using two spatially separated probes is accomplished by measuring the phase difference of the signals measured by each probe tip. For a single propagating plane wave, having the typical $\omega t-\vec{k} \cdot \vec{r}$ dependence and travelling past the two probes, a phase difference of $\cos \theta=\vec{k} \cdot \vec{r} /|k||r|$ occurs because of the finite transit time of the wave past the spatially separated probes. By knowing the probe separation distance, $|\vec{r}|$, and the measured phase difference, the wave number $k$ can be determined from the ratio of $\theta /|\vec{r}|$.

Determining the phase difference due to purely sinusoidal signals is relatively simple. However, the measured signals are typically a conglomeration of multiple frequencies with varying phase relationships, harmonics, and noise. For the measurements in this work, phase differences at specific frequencies are determined by calculating the cross-power spectrum of the two measured time series. The cross-power spectrum is the product of the Fast Fourier Transform (FFT) of one time series and the complex conjugate of the FFT of the second time series. To see this more explicitly, we first define the FFT's of the individual time series $f_{1}\left(x_{1}, t\right)$, and $f_{2}\left(x_{2}, t\right)$ to be

$$
\Phi_{1}\left(x_{1}, \omega\right)=\int_{-\infty}^{\infty} f_{1}\left(x_{1}, t\right) \cos (\omega t) d t-i \int_{-\infty}^{\infty} f_{1}\left(x_{1}, t\right) \sin (\omega t) d t
$$

and

$$
\Phi_{2}\left(x_{2}, \omega\right)=\int_{-\infty}^{\infty} f_{2}\left(x_{2}, t\right) \cos (\omega t) d t-i \int_{-\infty}^{\infty} f_{2}\left(x_{2}, t\right) \sin (\omega t) d t
$$

where $x_{1}$ and $x_{2}$ are the respective locations of the probes. Multiplying Equation (3.10) by the complex conjugate of Equation (3.11) produces the cross-power spectrum, 


$$
P_{12}(\Delta x, \omega)=\Phi_{1}\left(x_{1}, \omega\right) \Phi_{2}^{*}\left(x_{2}, \omega\right),
$$

where $\Delta x$ is the spatial separation of the probes. Further expansion of Equation (3.12), by substitution of Eqs. (3.10) and (3.11), yields the cross-power spectrum as a function of the real and imaginary parts of each FFT

$$
P_{12}(\Delta x, \omega)=\left(\varphi_{1 \mathrm{Re}} \varphi_{2 \mathrm{Re}}+\varphi_{1 \mathrm{Im}} \varphi_{2 \mathrm{Im}}\right)+i\left(\varphi_{1 \mathrm{Im}} \varphi_{2 \mathrm{Re}}-\varphi_{1 \mathrm{Re}} \varphi_{2 \mathrm{Im}}\right) .
$$

In the complex plane, the phase difference of the cross-power spectrum, and therefore the two time series, is just the angle between the "real vector" and the "imaginary vector"

$$
\Theta(\omega)=\tan ^{-1}\left(\frac{\left(\varphi_{1 \mathrm{Im}} \varphi_{2 \mathrm{Re}}-\varphi_{1 \mathrm{Re}} \varphi_{2 \mathrm{Im}}\right)}{\left(\varphi_{1 \mathrm{Re}} \varphi_{2 \mathrm{Re}}+\varphi_{1 \mathrm{II}} \varphi_{2 \mathrm{Im}}\right)}\right)
$$

An implicit assumption in these calculations is that the signals have a relatively large amplitude compared to the noise. When the signals have a low signal-to-noise ratio, ensemble averaging of many cross-power spectra can significantly improve the precision of the phase measurements (the errors decrease as $1 / \sqrt{M}$ where $M$ is the number of samples). ${ }^{14,15}$ An effective way to ensemble average several cross-power spectra is through a spectral density function $S(\omega, k)$ analysis. The spectral density function (SDF) is a function of the angular frequency, $\omega$, and the wave number, $k$ (obtained from the phase difference measurements) and is ${ }^{15}$

$$
S(n \Delta k, l \Delta \omega)=\frac{1}{M} \sum_{j=1}^{M} I_{n \Delta k}\left[k^{(j)}(l \Delta \omega)\right]\left(\frac{1}{2}\left(P_{1}^{(j)}(l \Delta \omega)+P_{2}^{(j)}(l \Delta \omega)\right)\right),
$$

where $\Delta k$ and $\Delta \omega$ are the wave number and frequency bin widths, $l$ is the number of frequency increments, $n$ is the number of wave number increments, $M$ is the number of 
ensembles to average, $P_{1}(l \Delta \omega)$ and $P_{2}(l \Delta \omega)$ are the individual time series power spectra, and the selection function, $I_{n \Delta k}\left[k^{(j)}(l \Delta \omega)\right]$ is $^{15}$

$$
I_{n \Delta k}\left[k^{(j)}(l \Delta \omega)\right]=\left[\begin{array}{cc}
1 & \left(n-\frac{1}{2}\right) \Delta k<k^{(j)}(l \Delta \omega)<\left(n+\frac{1}{2}\right) \Delta k \\
0 & \text { elsewhere }
\end{array}\right] .
$$

In essence, the SDF acts as a statistical histogram for the ensemble averaged spectral power contained in the individual FFT's. For each incremental frequency step and wave number the spectral power in each FFT is placed in the corresponding frequency-wave number bin. The result is a two dimensional array, comprised of the ensemble averaged spectral powers, for all frequencies and their associated wave numbers. A significant advantage of the SDF approach is the ability to identify several individual, or even a spectrum, of wave numbers associated with a given frequency.

The Joerger digitizer used for these experiments can acquire time series measurements of up to 262,144 points with digitation rates up to a maximum of 100 MHz. Typically, several time series were acquired for each probe tip for each measurement. All the individual time series for each probe tip were saved to binary files for post-acquisition analysis. Multiple time series of 262,144 points results in enourmous file sizes even if saved in a binary format. For example, the storage space required for 20 time series measurements from a single probe tip is approximately $40 \mathrm{MB}$. Therefore, a dedicated 1TB hard drive was purchased for storage of the electrostatic probe data.

With such long time series, it is possible to subdivide the individual time series sets into smaller intervals to increase the number of effective data sets for averaging. The noise reduction obtained by creating subsets and increasing the total number of ensembles for averaging often outweighs the loss in frequency resolution. For example, 
the normally distributed statistical noise in a data set with 10 time series for averaging and 262,144 points will only decrease by a factor of $\sim 1 / \sqrt{10}$. By subdividing the 10 individual time series into 32 subsets, producing time series of 8,192 points, the statistical noise is reduced by factor of $\sim 1 / \sqrt{320}$; an improvement of almost a factor of 6 . The effect on the reduction of time series points to 8,192 (thereby reducing the frequency resolution from approximately $380 \mathrm{~Hz}$ to $12,200 \mathrm{~Hz}$ ) for fluctuations of $100 \mathrm{kHz}$ or greater is negligible. See Table 3.1 for a complete list of possible subdivisions and the corresponding effect on the frequency resolution. For the time series measurements in this work, the typical number of subdivisions used was 16.

Table 3.1 Time series subdivision and frequency resolution.

\begin{tabular}{|c|c|c|}
\hline $\begin{array}{c}\mathrm{N} \\
\text { (number of subdivisions) }\end{array}$ & $\begin{array}{c}\text { Time Series } \\
\text { (record length) }\end{array}$ & $\begin{array}{c}\text { Frequency Resolution }[\mathrm{Hz}] \\
\text { (digitation rate }=100 \mathrm{MHz})\end{array}$ \\
\hline 1 & 262,144 & $\sim 381$ \\
\hline 2 & 131,072 & $\sim 762$ \\
\hline 4 & 65,536 & $1.53 \times 10^{3}$ \\
\hline 8 & 32,768 & $3.05 \times 10^{3}$ \\
\hline 16 & 16,384 & $6.10 \times 10^{3}$ \\
\hline 32 & 8,192 & $1.22 \times 10^{4}$ \\
\hline
\end{tabular}

One final point concerning spectral analysis concerns the use of windowing functions before calculating the FFT of a time series. Finite length records can artificially introduce high frequencies into an FFT due to the sharp cutoffs at the ends of the record. By applying a windowing function to a time series, producing a convolution of the window function and the time series reduces the finite record length effects while the dynamic range of the spectral analysis signal-to-noise can be increased. One drawback to applying a windowing function is spectral leakage. Depending on the specific type of window employed, spectral leakage can lead to an artificial broadening of peaks in frequency space, possibly obscuring signals close in frequency. An excellent discussion 
of the application of spectral analysis and windowing functions can be found in the Application Note found on the National Instruments website. ${ }^{16}$ An illustration of the effects on the signal-to-noise dynamic range due to different windowing functions, as provided by LabWindows, is shown in Figure 3.6.

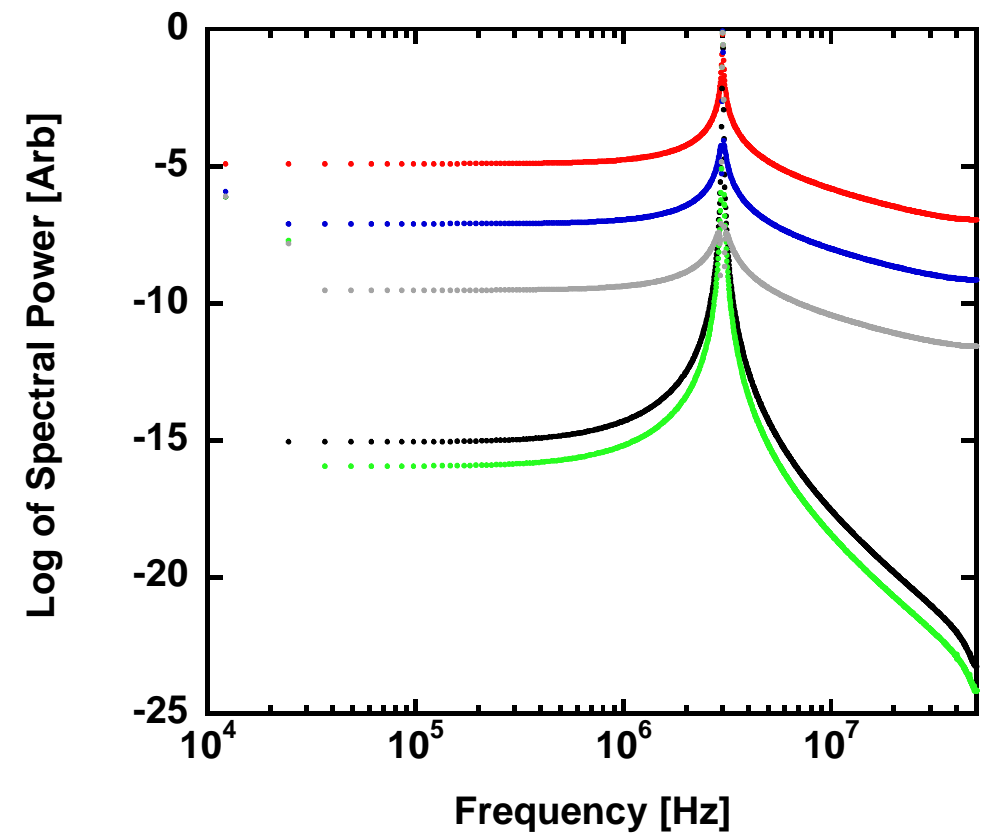

Figure 3.6 Windowing functions applied to pure sine wave of $3 \mathrm{MHz}$ digitized at a rate of $100 \mathrm{MHz}$ with 262,144 points divided into 32 subsets. The applied windows are: (_) No Window, ( $)$ Hanning, (_) Hamming, (_ Blackman, (_ $)$ Blackman-Harris.

As a test of the windowing functions available in LabWindows, a synthetic data array for a pure $3 \mathrm{MHz}$ sine wave was created with the same number of points that would be acquired in a typical experiment $(262,144)$ and at the typical digitation rate $(100 \mathrm{MHz})$ for 10 time series. The individual time series were subdivided into 32 subsets and the different windowing functions, as defined in Table 3.2, applied to the signals before the FFTs were averaged. Figure 3.6 shows the $\log$ of the spectral power as a function of frequency for each of the windowed $3 \mathrm{MHz}$ signals. When no window is applied, also known as a Flat Top window, the noise level is 5 orders of magnitude or more below the 
peak at $3 \mathrm{MHz}$. Applying the Blackman window reduces the noise level to approximately 16 orders of magnitude below the $3 \mathrm{MHz}$ peak. The other windowing functions applied to the $3 \mathrm{MHz}$ signal produce noise levels that lie between the flat top and Blackman windows. For these experiments reported here, the Blackman window was used for all measured time series.

Table 3.2 Windowing functions where $i$ is the index value and $n$ is the total number of points. ${ }^{17}$

\begin{tabular}{|c|c|}
\hline Window Function Name & Definition \\
\hline No Window (Flat Top) & 1 \\
\hline Hanning & $0.5-0.5 \cos \left(\frac{2 \pi i}{n}\right)$ \\
\hline Hamming & $0.54-0.46 \cos \left(\frac{2 \pi i}{n}\right)$ \\
\hline Blackman & $0.42-0.5 \cos \left(\frac{2 \pi i}{n}\right)+0.08 \cos \left(\frac{4 \pi i}{n}\right)$ \\
\hline Blackman-Harris & $0.42323-0.49755 \cos \left(\frac{2 \pi i}{n}\right)+0.07922 \cos \left(\frac{4 \pi i}{n}\right)$ \\
\hline
\end{tabular}

The primary algorithms used in the application of the SDF analysis method were originally written by Paul Keiter and John Kline based on the algorithms of Beall ${ }^{14}$ and Assadi. ${ }^{15}$ Modifications to the code for this work include the time series subdivision, windowing function options, and the ability to import binary formatted files. Figure 3.7 shows a typical SDF from the electrostatic double probe with all the laboratory equipment operating, including the rf amplifier, but without gas in the chamber. Application of the SDF analysis to the background noise data illustrates the SDF technique and provides an opportunity to review the intrinsic noise spectrum. For these data and all spectral density plots in this work, the frequency resolution chosen is $\Delta f \sim$ $6,103.5 \mathrm{~Hz}$ and the wave number resolution is $\Delta k \sim 0.68 \mathrm{rad} / \mathrm{cm}$. The wave number 
resolution is determined by $\Delta k=2 \pi /\left(d R_{\text {length }}\right)$ where $d$ is the probe tip separation and $R_{\text {length }}$ is the total number of wave number bins chosen for the SDF analysis. As a compromise between phase resolution and improved statistics, 151 wave number bins were used for the SDF analyses in this work.

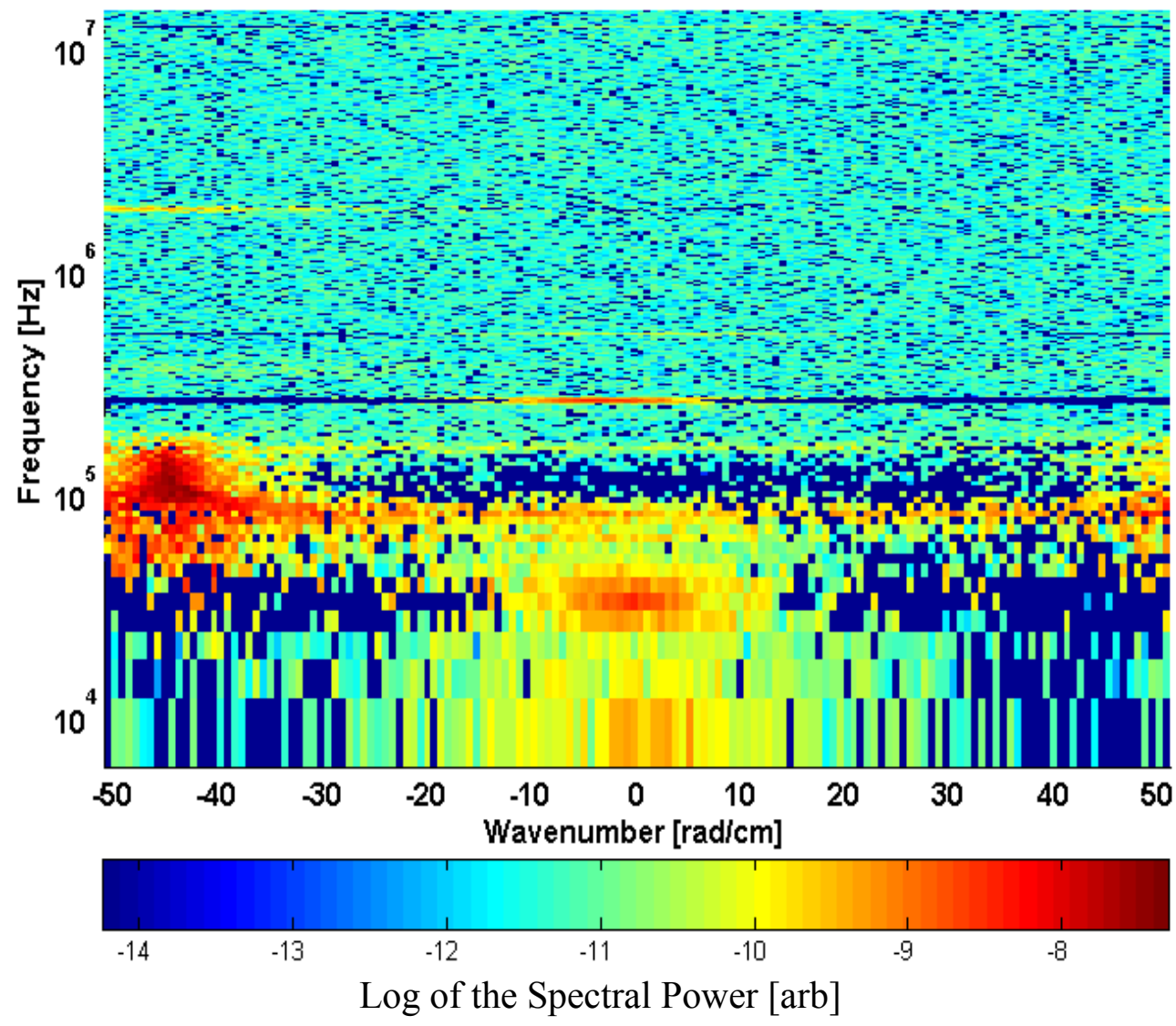

Figure 3.7 Surface plot of the spectral density with all the laboratory equipment operating, including the rf amplifier at $9.5 \mathrm{MHz}$, but with no gas in the chamber.

The spectral density shown in Figure 3.7 has several regions of significant noise. These large amplitude noise signals can conceal real waves, thus requiring some type of suppression. To suppress the noise, the frequency ranges containing the noise are replaced with the lowest signal amplitude within the individual data set. While narrow frequency bands of noise can be individually suppressed, the noise below $200 \mathrm{kHz}$ is broad enough and large enough that the entire frequency region is removed. The 
justification for removing the low frequency region, besides the limited frequency resolution, was the expectation that the frequencies of interest would be larger than 200 kHz. The spectral density, after the noise suppression, is shown in Figure 3.8. The change to note between Figures 3.7 and 3.8 is the overall reduction in the background amplitude by nearly two orders of magnitude. By reducing the relative noise amplitude, low power electrostatic fluctuations are easier to identify visually.

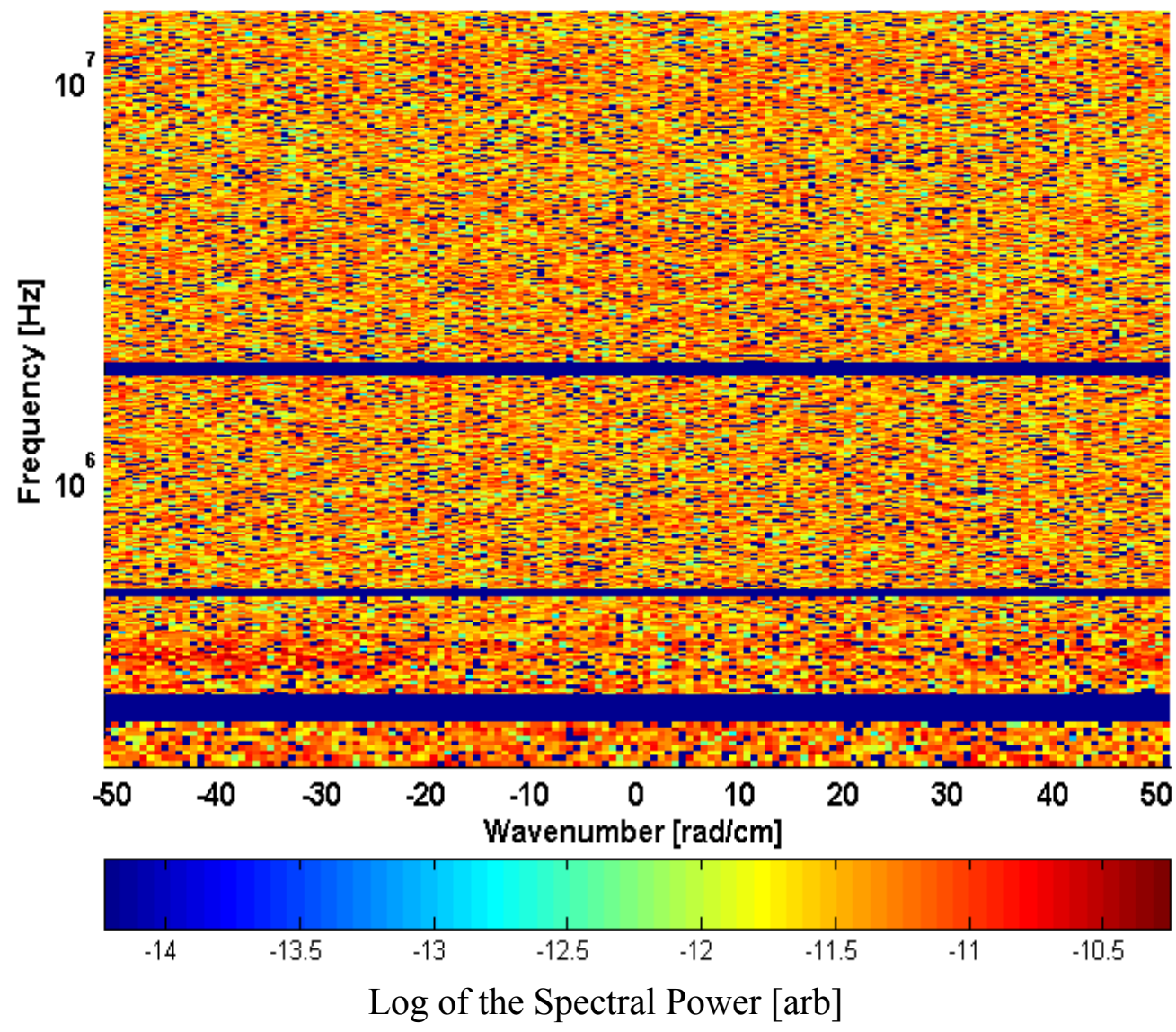

Figure 3.8 Surface plot of the spectral density with the noise suppression applied to the data in Figure 3.7.

Due to the finite binning nature of the spectral density function, determining the wave number or frequency of a specific fluctuation requires additional analysis. Using an experimental data set, shown in Figure 3.9, the process of determining a fluctuation wave number is described below; the details of the fluctuation are discussed later in this dissertation. In Figure 3.9, the frequency range is limited to $3 \mathrm{MHz}$ to highlight the 
particular fluctuation of interest. A single frequency slice through the spectral density at $400 \mathrm{kHz}$ is shown in Figure 3.10 .

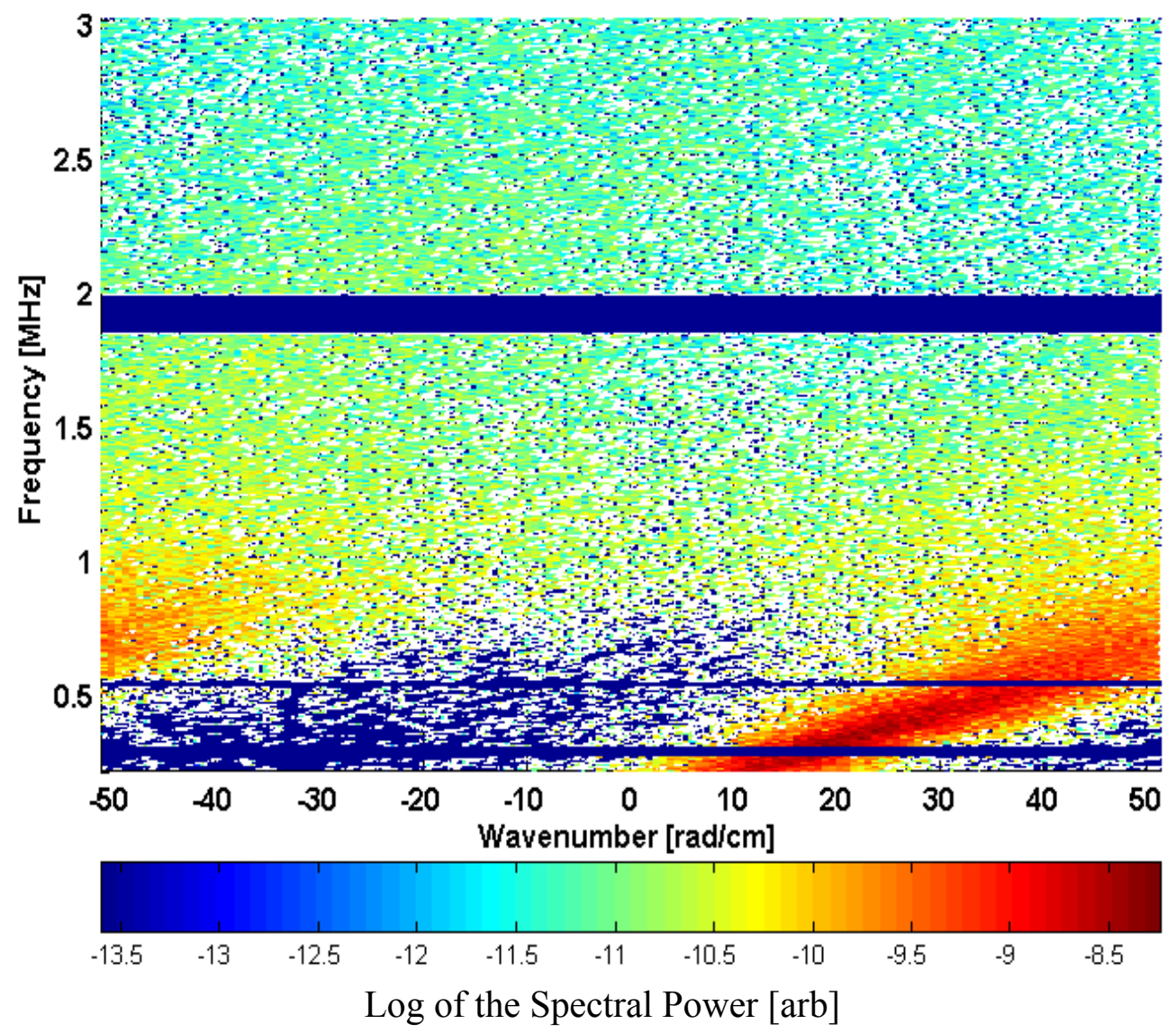

Figure 3.9 The spectral density versus frequency and wave number at an rf frequency of $9.5 \mathrm{MHz}$ and 950 Gauss for the electrostatic probe aligned perpendicular to the magnetic field.

Figure 3.10(a) shows the distribution of the spectral power contained in the 400 $\mathrm{kHz}$ bin as a function of wave number. The distribution of the spectral density has a finite wave number width and requires a Gaussian fit to determine the mean wave number and standard deviation $\left(\Delta k_{\sigma}\right)$. The mean wave number obtained from the fit is $27.0 \pm 0.2$ $\mathrm{rad} / \mathrm{cm}$ with $\Delta k_{\sigma} 4.2 \pm 0.3 \mathrm{rad} / \mathrm{cm}$. To reduce the noise in the single frequency cut (variation in the amplitudes relative to the fit), a sum of frequency bins around the frequency of interest can be used to improve the signal-to-noise. Fig 3.10(b) shows the 
integrated spectral power over a width of $\sim 67 \mathrm{kHz}$ centered at $400 \mathrm{kHz}$. The frequency averaged data have a mean wave number of $27.3 \pm 0.1 \mathrm{rad} / \mathrm{cm}$ and $\Delta k_{\sigma} 4.3 \pm 0.1 \mathrm{rad} / \mathrm{cm}$.
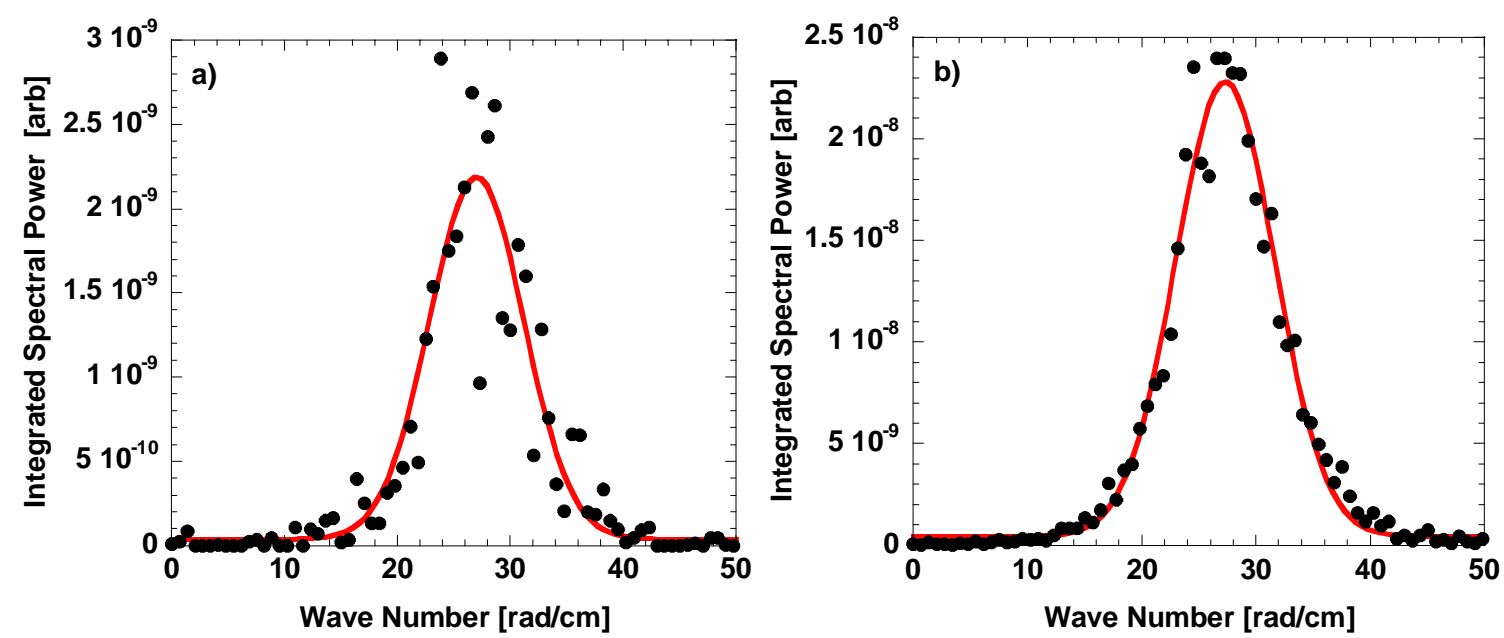

Figure 3.10 Wave number spectra from a (a) single frequency slice at $400 \mathrm{kHz}$, and (b) after summing a frequency window of $\sim 67 \mathrm{kHz}$ around the $400 \mathrm{kHz}$ slice. The red line is the Gaussian fit.

When using spatially fixed probes in conjunction with cross-power spectral techniques, one needs to consider the effects of aliasing in both the frequency and spatial domains. In the frequency domain, aliasing occurs when a periodic signal has a frequency that exceeds the Nyquist frequency $\left(f_{\text {Nyquist }}=1 / 2\right.$ Sampling Rate) and for a sampling rate of $100 \mathrm{MHz}$ the Nyquist frequency is $50 \mathrm{MHz}$ for these experiments. No obvious frequency aliasing was detected in this work. In the spatial domain, aliasing occurs when the fluctuation wavelength becomes smaller than the probe separation. An illustration of spatial aliasing is shown in Figure 3.11, where the same measured phase difference is obtained for two different wavelengths. What is shown on the left hand side of Figure 3.11 is the lowest resolvable wavelength measurement for a fixed probe pair; $\lambda_{\min }=2 d$, where $d$ is the probe separation distance. With a probe tip separation of $0.061 \mathrm{~cm}$, wavelengths on the order of $0.12 \mathrm{~cm}\left(k_{\max }\right.$ of $\left.\sim 51.5 \mathrm{rad} / \mathrm{cm}\right)$ are measureable. However, in some cases, spatial aliasing can be easily detected and larger wave number measurements 
recovered. This typically occurs when a broad spectrum, in wave number and frequency, is visible in the spectral density measurement.
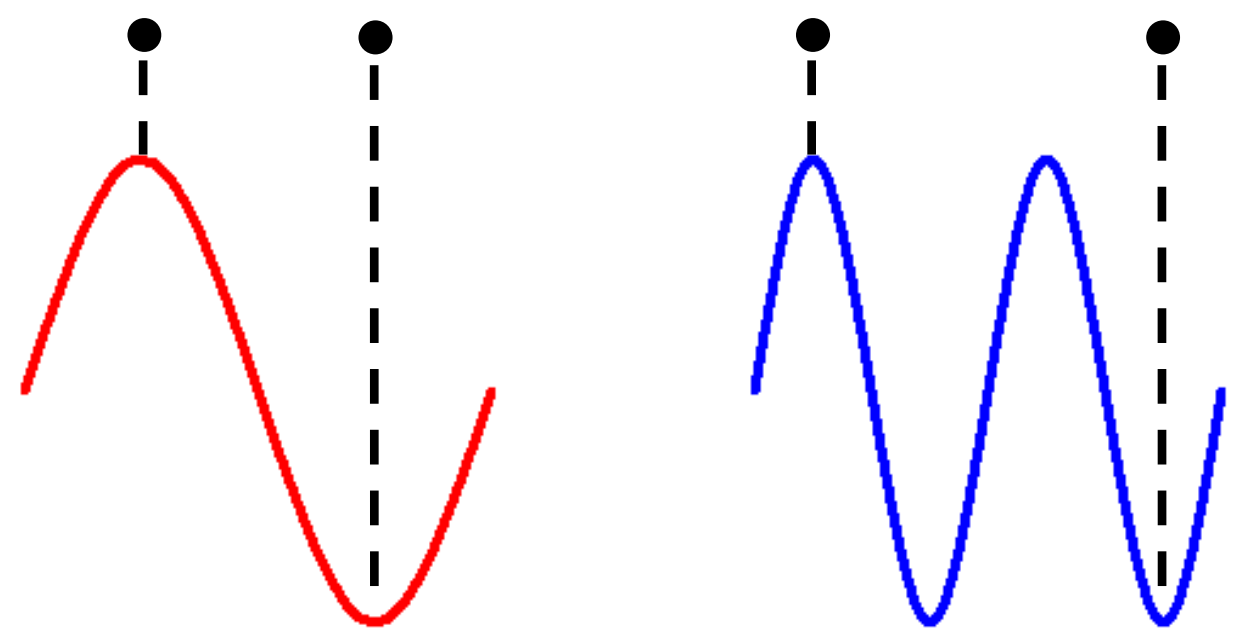

Figure 3.11 An illustration of spatial aliasing for a fixed probe pair. The black dots and dashed lines represent the probe tips while the color sinusoids represent the wave amplitudes at an instant in time.

\subsection{Laser Induced Fluorescence (LIF)}

Although not explicitly used in this work, some of the motivation for these experiments comes from Laser-induced-fluorescence (LIF) measurements of ion temperatures. LIF is a widely-used spatially resolved, non-invasive method of measuring particle velocity distribution functions. In 1975, Stern and Johnson were the first to perform LIF in plasma, using a single frequency argon ion laser. ${ }^{18} \mathrm{~A}$ tunable dye laser was first used for LIF in plasma in 1979 by Meng and Kunze, ${ }^{19}$ however Hill, Fornaca, and Wickham first used the ability to tune the laser for "velocity selective" LIF in $1983 .{ }^{20}$ Rather than measuring the emission line shape, which is limited by the resolution of the spectrometer used, LIF measures the absorption line shape and the velocity resolution is typically determined by the linewidth of the tunable laser. The dye laser has been the laser of choice for LIF for many years due to its tunability, high output power, and broad 
wavelength range. Recent advances in diode based laser systems have allowed for cheaper and more compact LIF systems. ${ }^{21}$

\subsubsection{LIF Theory}

LIF essentially measures the particle velocity distribution function through the Doppler effect. An ion or atom having an accessible energy state is excited by the laser and then spontaneously fluoresces as the excited state decays to a lower energy level. Comprehensive discussions of LIF and the individual broadening mechanisms relevant for LIF IN HELIX can be found in Ref. [11] and Ref. [22].

In a typical LIF measurement, the frequency of a very narrow bandwidth laser is swept across a collection of ions or atoms that have a thermally broadened velocity distribution. Typically the ions or atoms are assumed to obey a Maxwellian velocity distribution is given by

$$
f(\mathrm{v})=A \exp \left(\frac{-m\left(\mathrm{v}-\mathrm{v}_{0}\right)^{2}}{2 k_{b} T_{i}}\right)
$$

where $m$ is the species mass, $v_{0}$ is the average bulk flow speed of the entire distribution, $k_{b}$ is Boltzmann's constant, and $T_{i}$ is the temperature of the ions. In an argon plasma, excitation out of the argon ion metastable state, $3 \mathrm{~d}^{2} \mathrm{G}_{9 / 2} \rightarrow 4 \mathrm{p}^{2} \mathrm{~F}_{7 / 2}$ transition, is accomplished with a laser wavelength tuned to $611.5 \mathrm{~nm}$. Decay from the excited state to a lower state, $4 \mathrm{p}^{2} \mathrm{~F}_{7 / 2} \rightarrow 4 \mathrm{~s}^{2} \mathrm{D}_{5 / 2}$, is accompanied by the emission of photons at a wavelength of $461.0 \mathrm{~nm}$. A graphical representation of the absorption and fluorescence emission is shown in Figure 3.12. 


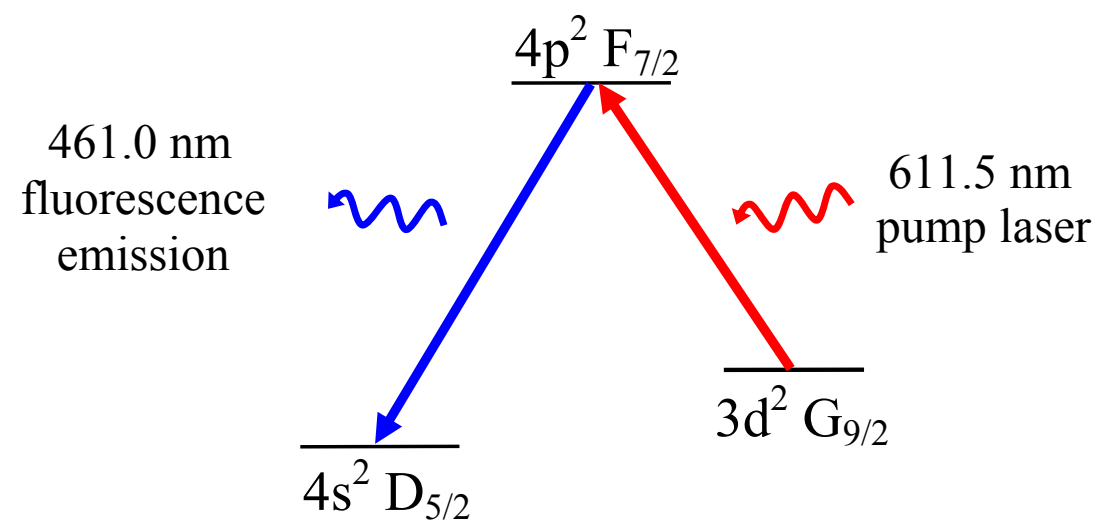

Figure 3.12 Three level LIF scheme used to measure argon ion velocity distribution functions.

\subsubsection{LIF Apparatus}

The LIF laser system consists of a $6 \mathrm{~W}$ Coherent Innova 300 argon-ion laser that pumps a Coherent 899 ring dye laser. A $10 \%$ beam splitter directs a portion of the beam through an iodine cell and into a Burleigh 1500 wavemeter. Over the range of wavelengths used for argon LIF measurements, the iodine cell has numerous absorption lines. ${ }^{23,24}$ The spontaneous fluorescence from the optically pumped excited states in the iodine cell is measured with a photodiode detector and recorded for each LIF measurement. Since the iodine cell is relatively cold and is at rest in the laboratory frame, the pattern of absorption lines occurs at the same laser frequency for every measurement. Therefore, the iodine cell measurements provide a zero velocity (rest frame) reference for each LIF measurement in the plasma. With the iodine cell reference, bulk ion flows on the order of $25 \mathrm{~m} / \mathrm{s}$ can be reliably measured in argon plasmas. The Burleigh 1500 wavemeter is used for tuning the dye laser to the correct wavelength. The dye laser frequency sweep is controlled by a \pm 5 volt signal. The laser frequency may be swept over frequency range up to $25 \mathrm{GHz}$, roughly $0.03 \mathrm{~nm}$. Fluorescent emission from the 
upper level was measured with a filtered photomultiplier tube detector. The filter in front of the photomultiplier has a 1 nanometer wide bandpass centered on the $461.0 \mathrm{~nm}$ emission line. The output of the dye laser was chopped at approximately 1 to $2 \mathrm{kHz}$ with a mechanical chopper. A reference signal from the chopper controller was sent to the Stanford Research SR830 lockin amplifier that monitors the photomultiplier tube signal. The lock-in amplifier extracts the fluorescence signal from the intense background emission at the same wavelength. After the laser light passes through the mechanical chopper, it is coupled into a fiber optic cable with a fiber coupler. The fiber optic cable transports the laser light from the laser laboratory to HELIX, where several sets of injection optics are mounted on the HELIX chamber. The collected light is also transported to the photomultiplier detector by fiber optic cable. This configuration enables LIF measurements to be performed at different locations by simply moving the fiber optic cables. No other changes to the experimental hardware or data acquisition apparatus are required. The collection optics are optimized to collect the maximum amount of light through a 1" lens while still matching the numerical aperture of the lenses to the collection fiber optic cable.

In addition to the iodine cell photodiode signal, the output of the SR830 lock-in amplifier, the output power level signal from the ring dye laser, and the sweep voltage sent to the dye laser controller are recorded simultaneously for each scan of the dye laser frequency. The digitizer is controlled through a custom LabWindows ${ }^{\mathrm{TM}}$ interface and the data stored on a PC. The output signal from the SR830 lock-in amplifier was normalized to the laser power output signal to account for variations in the laser power during the 
sweep of the laser frequency. Depending on the signal level, LIF measurements are often averaged together to improve signal to noise levels.

\subsubsection{LIF Data Analysis}

A typical measurement of a perpendicular ion velocity distribution in a HELIX argon plasma is shown in Figure 3.13. The ion temperature and the peak amplitude frequency of the velocity distribution are determined via a nonlinear fit with the data to a Doppler broadened absorption lineshape for an argon plasma;

$$
I(v)=I_{0} \exp \left(-0.0779\left(v-v_{0}\right)^{2} / T_{i}\right)
$$

where $T_{i}$ is the ion temperature in $\mathrm{eV}$ and $v_{0}$ is the center frequency in $\mathrm{GHz}$. Shifts in the center frequency of the distribution relative to the reference iodine transitions provide a measure of the absolute drift velocity of the ions.

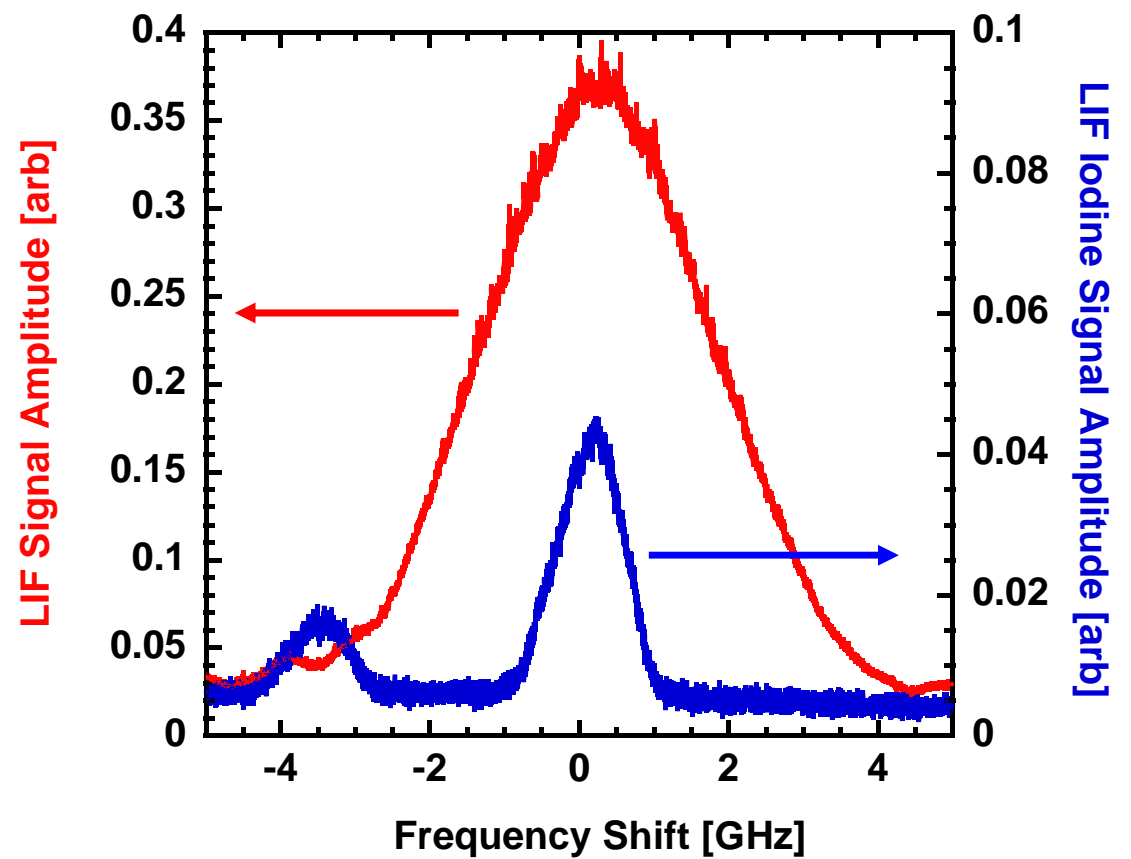

Figure 3.13 The perpendicular ion velocity distribution function (red) with $\mathrm{T}_{\mathrm{i} \perp}=0.34 \mathrm{eV}$, as measured with LIF in HELIX with the corresponding iodine trace (blue) used for a zero flow reference. 


\section{Chapter 3 References}

${ }^{1}$ I.H. Hutchinson, Principles of Plasma Diagnostics, (Cambridge University Press, Cambridge, 1987).

${ }^{2}$ L. Schott, in Plasma Diagnostics, ed. by W. Lochte-Holtgreven (John Wiley, New York, 1968).

${ }^{3}$ F.F. Chen, in Plasma Diagnostic Techniques, ed. by R.H. Huddlestone and S.L. Leonard (Academic Press, New York, 1965).

${ }^{4}$ N. Hershkowitz, How Langmuir Probes Work, in Plasma Diagnostics, Discharge Parameters and Chemistry, ed. by O. Aucillo and D.L. Flamm (Academic Press, 1989).

${ }^{5}$ V.I. Demidov, S.V. Ratynskaia, and K. Rypdal, Rev. Sci. Instrum. 73, 3409 (2002).

${ }^{6}$ P.A. Keiter et al., Phys. Plasmas 7, 779 (2000).

${ }^{7}$ J.L. Kline, Slow Wave Ion Heating and Parametric Instabilities in the HELIX Helicon Source, Ph.D. Dissertation, West Virginia University, Morgantown, (2002).

${ }^{8}$ N.A. Krall and A.W. Trivelpiece, Principles of Plasma Physics, (San Francisco Press, San Francisco, 1986).

${ }^{9}$ I.D. Sudit and F.F. Chen, Plasma Sour. Sci. Technol. 3, 162 (1994).

${ }^{10}$ Lenox-Fugle International, Inc. 1071 N. Grand Ave. \#115, Nogales, AZ 85621. (520) 287-5847.

${ }^{11}$ A.M. Keesee, Neutral Density Profiles in Argon Helicon Plasmas, Ph.D. Dissertation, West Virginia University, Morgantown, (2006).

${ }^{12}$ P.A. Keiter, Experimental Investigation of Ion Temperature Anisotropy Driven Instabilities in a High Beta Plasma, Ph.D. Dissertation, West Virginia University, Morgantown (1999).

${ }^{13}$ M.J. Burin, G.R. Tynan, G.Y. Antar, N.A. Crocker, and C. Holland, Phys. Plasmas, 12, 052320 (2005).

${ }^{14}$ J.M. Beall, Y.C. Kim, and E.J. Powers, J. Appl. Phys., 53, 3933 (1982).

${ }^{15}$ S. Assadi, Measurement of Magnetic Turbulence Structure and Nonlinear Mode Coupling of Tearing Fluctuations in the Madison Symmetric Torus Reversed Field Pinch, Ph D. Thesis, University of Wisconsin - Madison, Madison (1994).

${ }^{16}$ A.F. Harvey and M. Cerna, The Funcdamentals of FFT-Based Signal Analysis and Measurement in LabVIEW and LabWindows, Application Note 041 (1993).

${ }^{17}$ www.ni.com.

${ }^{18}$ R.A. Stern and J.A. Johnson, Phys. Rev. Lett. 34, 1548 (1975).

${ }^{19}$ H.C. Meng and H.-J. Kunze, Phys. Fluids 22, 1082 (1979).

${ }^{20}$ D.N. Hill, S. Fornaca, and M.G. Wickham, Rev. Sci. Instrum. 54, 309 (1983).

${ }^{21}$ R.F. Boivin and E.E. Scime, Rev. Sci. Instrum. 74, 4352 (2003).

${ }^{22}$ R.F. Boivin, Line Broadening Mechanisms, PL-039, West Virginia University, Morgantown (1999).

${ }^{23}$ S. Gerstenkorn and P. Luc, Absolute Iodine $\left(\mathrm{I}_{2}\right)$ Standards Measured by Means of Fourier Transform Spectroscopy, Revue de Physique Appliquee, 14, 791 (1979).

${ }^{24}$ S. Gerstenkorn and P. Luc, Atlas du Spectre D'Absorption de la Molecule de L'Iodine Entre $14800-$ $20000 \mathrm{~cm}^{-1}$, (Editions du C.N.R.S., 15, quai Anatole-France, 75700 Paris) (1980). 


\section{Chapter 4: Cold Plasma Theory of the Helicon Plasma Source}

As noted earlier, the primary motivation of this work is to determine if short wavelength fluctuations, slow waves, are excited at the same conditions at which enhanced ion heating is observed in helicon sources. Solving the cold plasma dispersion relationship including ion terms and collisions, Cho found two solutions for typical helicon operating parameters. ${ }^{1}$ The first solution is the fast, or the helicon, wave; a bounded, right hand circularly polarized electromagnetic wave, generally known as a whistler wave. Several experimental measurements have confirmed that electromagnetic waves with wave fields consistent with helicon waves propagate downstream of the $\mathrm{rf}$ antenna in helicon sources. ${ }^{2,3}$ The second solution is the slow wave or Trivelpiece-Gould (TG) mode. The TG wave is so named because it is the same root of the cold plasma dispersion function that was identified by Trivelpiece and Gould in a pure electron, bounded plasma. ${ }^{4}$ The slow wave is a surface wave and is heavily damped by collisions, preventing it from propagating towards the center of the plasma. ${ }^{5}$ Near the lower hybrid resonance, the slow wave becomes almost completely electrostatic and attempts to directly measure the slow wave in a helicon plasma source have been hampered by the extremely short wavelength of the wave; expected wavelengths are on the order of $1 \mathrm{~mm}$ or smaller. High frequency current profile measurements provided preliminary evidence of slow wave excitation, but no definitive experiment observations have been reported. ${ }^{6}$ The cold plasma dispersion relation as applicable to the WVU helicon plasma source is reviewed in section 4.1. In section 4.2, the dispersion relation is developed into a predictive model for use in conjunction with the $300 \mathrm{GHz}$ CTS diagnostic. 


\subsection{The Cold Plasma Dispersion Relation}

Kline's application of the cold plasma dispersion model, as shown in Figure 1.6, suggested a correlation between slow wave excitation and associated ion heating in the WVU helicon source. ${ }^{7}$ For completeness, the cold plasma dispersion relation is reviewed here so that it can be employed later in a code for predicting the approximate plasma parameters and spatial location of the measureable wave number range for the $300 \mathrm{GHz}$ CTS diagnostic.

The cold plasma dispersion relation for helicon parameters is

$$
\rho^{4}-(\alpha+\beta) \rho^{2}+\alpha \beta-\gamma \delta=0
$$

where $\rho \equiv \frac{k_{\perp} c}{\omega}$ and $\alpha, \beta, \gamma$, and $\delta$ are given by

$$
\begin{aligned}
& \alpha=\varepsilon_{1}-N^{2}-\frac{\varepsilon_{2}^{2}}{\varepsilon_{1}}, \\
& \beta=\varepsilon_{3}\left(1-\frac{N^{2}}{\varepsilon_{1}}\right), \\
& \gamma=\frac{N \varepsilon_{3} \varepsilon_{2}}{\varepsilon_{1}} \\
& \delta=\frac{N \varepsilon_{2}}{\varepsilon_{1}} .
\end{aligned}
$$

$N \equiv \frac{k_{\|} c}{\omega}, C$ is the speed of light, $\omega$ is the wave frequency, and $k_{\perp}$ and $k_{\|}$are the respective wave numbers perpendicular and parallel relative to the applied magnetic field. $\varepsilon_{1}, \varepsilon_{2}$, and $\varepsilon_{3}$ are the cold plasma dielectric tensor components given by: 


$$
\begin{aligned}
& \varepsilon_{1}=1+\sum_{j=e, i} \frac{\omega_{p j}^{2}\left(1+i v_{j} / \omega\right)}{\omega_{c j}^{2}-\omega^{2}\left(1+i v_{j} / \omega\right)^{2}}, \\
& \varepsilon_{2}=\sum_{j=e, i} \frac{\sigma_{j} \omega_{p j}^{2}\left(\omega_{c j} / \omega\right)}{\omega_{c j}^{2}-\omega^{2}\left(1+i v_{j} / \omega\right)^{2}}, \\
& \varepsilon_{3}=1-\sum_{j=e, i} \frac{\omega_{p j}^{2}}{\omega_{c j}^{2}-\omega^{2}\left(1+i v_{j} / \omega\right)},
\end{aligned}
$$

where $v_{j}=v_{j n}+v_{j j}$ is the total collision frequency including ion-ion, ion-neutral, electron-electron, and electron-neutral collisions. The subscript $j$ represents the individual species, $\omega_{p j}$ is the plasma frequency, $\omega_{c j}$ is the cyclotron frequency, and $\sigma_{j}$ is the sign of the charge.

Figure 4.1 shows a series of perpendicular wave number solutions for the cold plasma dispersion relation for the typical helicon source parameters described in the figure caption. For low magnetic fields, the real wave number solutions are identical. As the magnetic field strength increases the solutions bifurcate into the fast wave and the slow wave. The perpendicular wave number of the fast helicon wave asymptotically approaches zero as the magnetic field increases. On the other hand, the perpendicular wave number of the slow wave solution continues to grow as the magnetic field is increased until the lower hybrid resonance is reached. The lower hybrid resonance occurs when $\varepsilon_{1}$ in the dispersion relation goes to zero, i.e. when the wave frequency $(\omega)$ is equal to $\omega_{L H}$. At $\omega=\omega_{L H}$, the perpendicular wave number would become infinite in a collisionless plasma. The lower hybrid frequency,

$$
\frac{1}{\omega_{L H}^{2}}=\frac{1}{\omega_{c e} \omega_{c i}}+\frac{1}{\omega_{p i}^{2}+\omega_{c i}^{2}},
$$


depends on the ambient magnetic field strength and also the plasma density in low density plasma. The inclusion of collisions reduces the wave number at $\omega=\omega_{L H}$ to a finite number, while also slightly shifting the resonance frequency. The important physics that occurs at the resonance is that the wave becomes almost completely electrostatic $(\vec{k} \| \tilde{E})$ and the phase velocity, $V_{p}=\omega / k_{\perp}$, becomes small. If the phase velocity becomes comparable to the ion thermal speed, the slow wave could damp on the ions, thus preferentially heating the ions in the perpendicular direction. Note that the fast wave can play no direct role in heating the ions due to its small perpendicular wave number (large phase velocity) and lack of any resonance. Thus, any perpendicular ion heating, as indicated by Figure 1.6, is assumed to be attributed to the damping of the slow wave on the ions or possibly another parametrically driven ion heating mechanism.

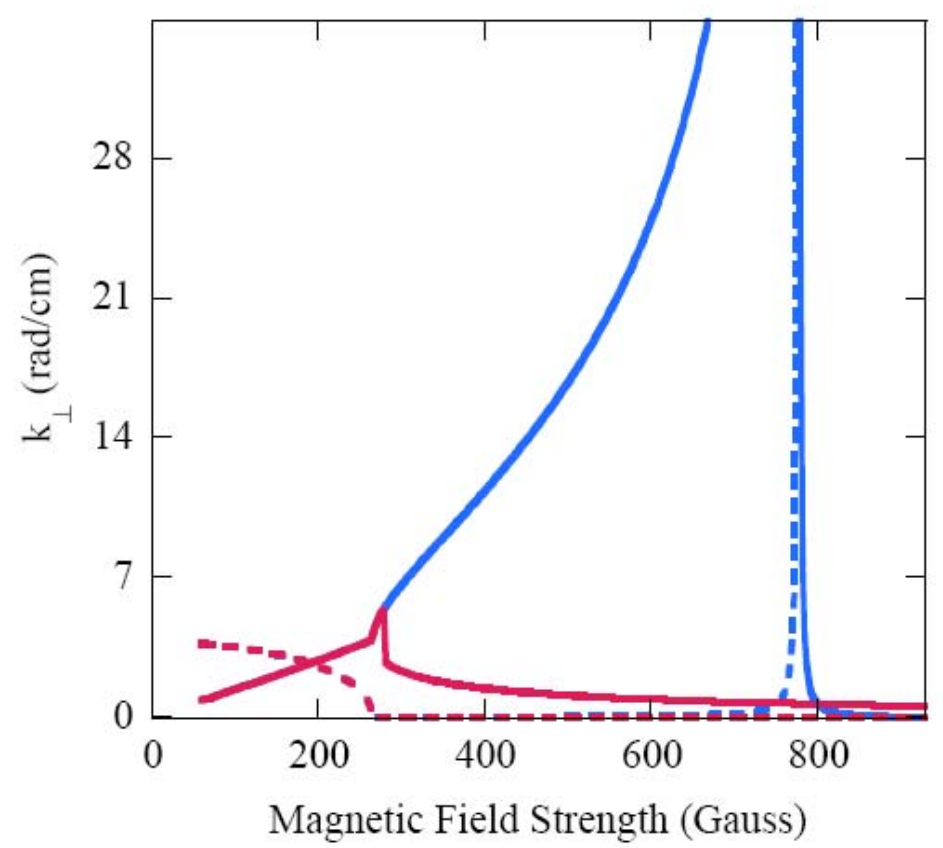

Figure 4.1 Solutions of the cold plasma dispersion relation for $k_{\perp}$ with $n=5 \times 10^{12} \mathrm{~cm}^{-3}, k_{\|}=0.26 \mathrm{rad} / \mathrm{cm}, \mathrm{f}$ $=9 \mathrm{MHz}$, and no collisions. ( $(-)$ is the absolute value of the real $k_{\perp}$ for the slow wave, $\left(\mathrm{n}_{-}\right)$is the absolute value of the imaginary $k_{\perp}$ for the slow wave, ( $)$ is the absolute value of the real $k_{\perp}$ for the fast wave, and ( $\ldots$ - ) is the absolute value of the imaginary $k_{\perp}$ for the fast wave. ${ }^{7}$ 
The imaginary wave numbers, a measure of the wave damping rates, are also identical for low magnetic field strengths. Note that although the imaginary solutions are shown as positive, which would indicate wave growth, they are actually negative and indicate damping. The wave damping for the slow wave solution is a maximum at the lower hybrid resonance. In contrast, the damping of the fast wave is finite, but small across the lower hybrid resonance.

While examining the computational cold plasma dispersion model used by Kline, two errors were discovered. The first error, which had only a minor effect, was a sign error in the $\varepsilon_{3}$ term of the dielectric tensor. Since $\varepsilon_{3}$ is the parallel component of the dielectric tensor, the impact on solutions for the perpendicular wave number was negligible. The second error was the explicit assumption that the fast and slow wave roots of the cold plasma dispersion relation always corresponded to the same root. Because the dispersion relation is $4^{\text {th }}$ order, there are always four roots. Since there are no odd powers, the solutions should always be paired with their respective complex conjugate, resulting in only two distinct solutions. The problem in the original computational model is that the solutions were selected a priori, without determining if the solution corresponded to the fast or slow wave. The revised model code, provided in Appendix B, makes no assumptions as to which solutions are the fast or slow waves. For each series of roots at a given set of parameters the slow wave and fast wave solutions are determined by explicitly calculating the magnitude of the wave number solution and sorting the solutions into the appropriate mode.

The revised model code produces a 3-D matrix of perpendicular wave number solutions for a range of magnetic fields, rf frequencies, and radii (corresponding to 
different plasma densities). By using the 3-D matrix method, we can examine several results simultaneously while only running the model code once. Figures 4.2 and 4.3 show the comparisons between the model code in Kline $e^{7}$ and the revised model for peak densities of $5 \times 10^{12} \mathrm{~cm}^{-3}$ and $2 \times 10^{13} \mathrm{~cm}^{-3}$ for a range of rf frequencies and magnetic fields. The radial density profile used for comparison has the form $n(r)=n_{0}\left(1-(r / a)^{2}\right)^{5}+n_{0} / 100$, which is believed to be the same as used in the original model. The dispersion relation solutions are normalized to the ion thermal velocity for a perpendicular ion temperature of $0.2 \mathrm{eV}$. It is advantageous to think of the normalized wave numbers as the ratio of the ion thermal velocity $\left(V_{t h i}\right)$ to the phase velocity $\left(V_{p}\right)$, $V_{t h i} k_{\perp} / \omega=V_{t h i} / V_{p}$. In this form, it is easier to identify the potential for wave-particle interactions.

The first noticeable difference between the figures is the amplitudes of the normalized wave numbers. The revised model produces normalized wave numbers on the order of four times the original model. The largest normalized wave numbers for the revised model in the lower density case (Figure 4.2) are approaching a value of one. This is a significant difference because as the ratio of the thermal velocity to normalized wave numbers increases toward a value of one, ion Landau damping of the slow wave can play a more significant role in ion heating. Compared to the original model, the overall increase through the entire parameter range is believed to be a combined result of a slightly different radial density profile, particularly near the lower density edge, and the more correct wave number solution choice. For the higher density case (Figure 4.3), the normalized wave numbers are still larger in the revised model, but are limited to a maximum value of 0.37 . 


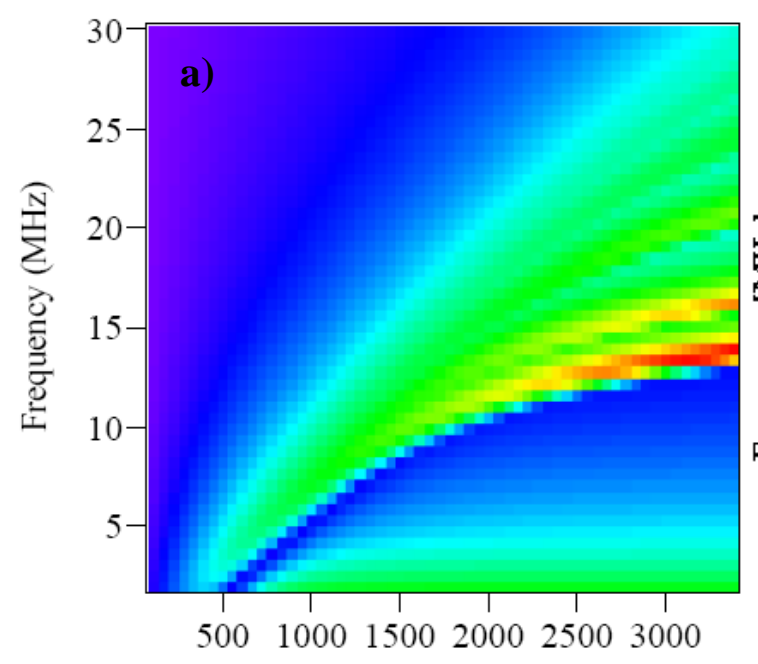

Magnetic Field Strength (Gauss)

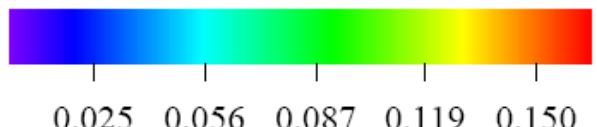

Normalized Wave Numbers $\left(\mathrm{V}_{\text {thi }} k_{\perp} / \omega\right)$

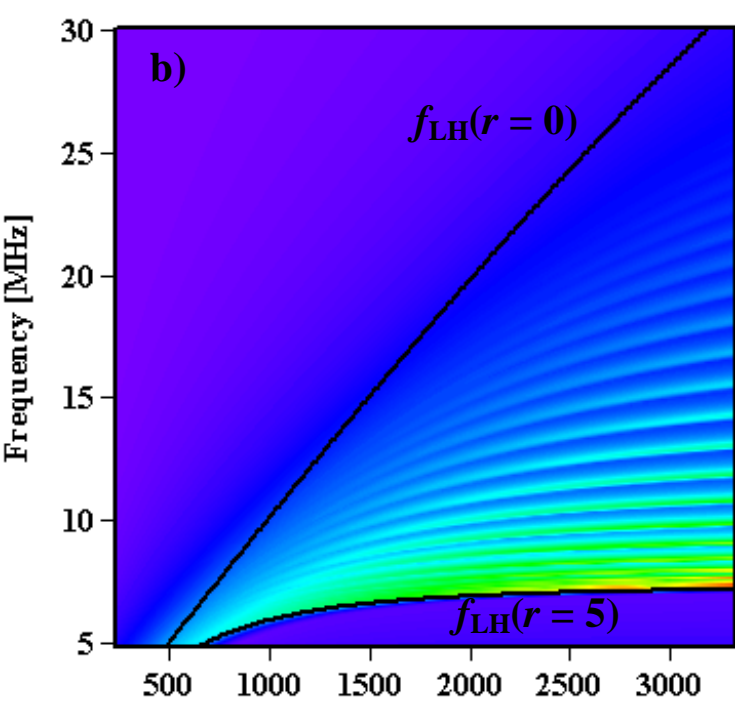

Magnetic Field [G]

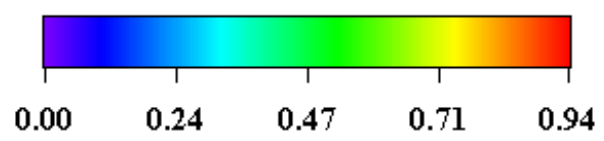

Normalized Wave Number $\left(\mathrm{V}_{\text {thi }} k_{\perp} / \omega\right)$

Figure 4.2 Normalized maximum values of the real component of $k_{\perp}$ as a function of rf driving frequency and magnetic field for a peak density of $5 \times 10^{12} \mathrm{~cm}^{-3}$, neutral pressure of 6.7 mTorr, electron temperature of $3 \mathrm{eV}$, ion temperature of $0.2 \mathrm{eV}$, and $k_{\|}$of $0.3 \mathrm{rad} / \mathrm{cm}$. a) Original model code. b) Revised model code with the black lines showing the calculated $f_{\mathrm{LH}}$ at the indicated radius.

Another significant difference between the original and revised models is the frequencies of the peak normalized wave numbers. Because the largest normalized wave numbers are associated with the lower hybrid resonance, the lower hybrid frequency is calculated at each radial location to provide a visual comparison to the wave numbers. The black lines overlaid in Figures $4.2 \mathrm{~b}$ and $4.3 \mathrm{~b}$ show the lower hybrid frequency as a function of the magnetic field for the density on axis $(r=0 \mathrm{~cm})$ and the edge density $(r=$ $5 \mathrm{~cm})$. In both cases, the lower hybrid frequencies on axis are essentially equivalent and scale linearly with increasing magnetic field. This is a result of the lower hybrid frequency, at high densities $\left(\mathrm{n} \sim 10^{12}-10^{13} \mathrm{~cm}^{-3}\right)$, depending only on the magnetic field $\omega_{L H} \approx \sqrt{\omega_{c e} \omega_{c i}} \propto B$. As the density decreases radially, the lower hybrid frequency 
becomes more dependant on the density, thereby decreasing the lower hybrid frequency. Because there is such a large difference between the peak density and the edge density, the reduction of the lower hybrid frequency at the edge is very clear in both cases. Additionally, the lower frequency peaks seen in the original model vanish in the revised model and are believed to have been an artifact of the method of solution selection in the original model.
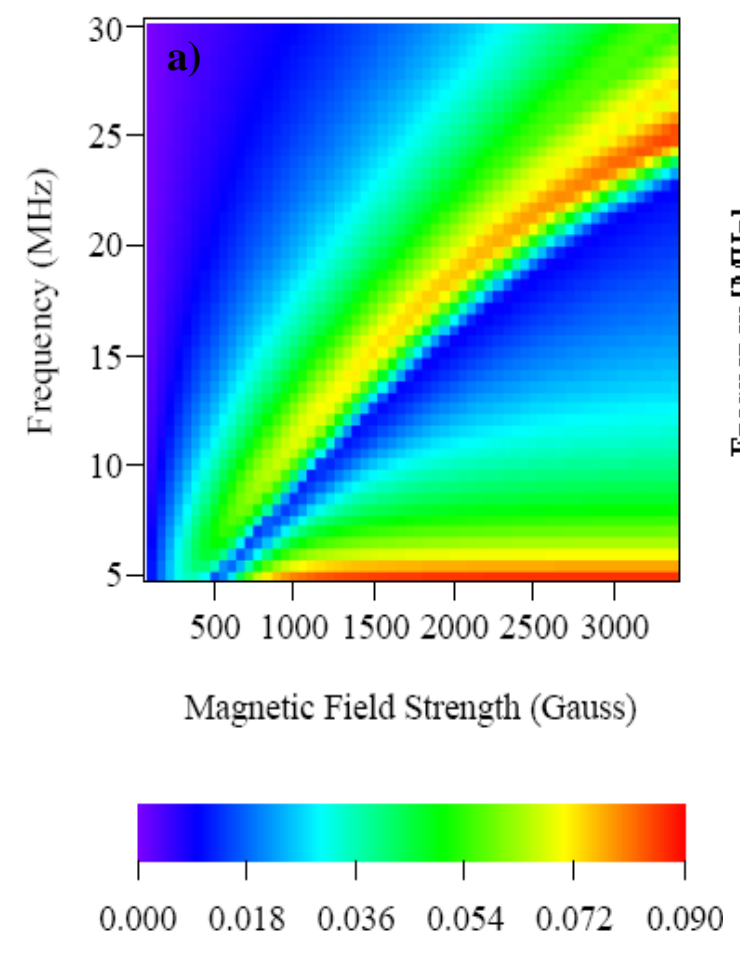

Normalized Wave Number $\left(\mathrm{v}_{\text {thi }} k_{\perp} / \omega\right)$

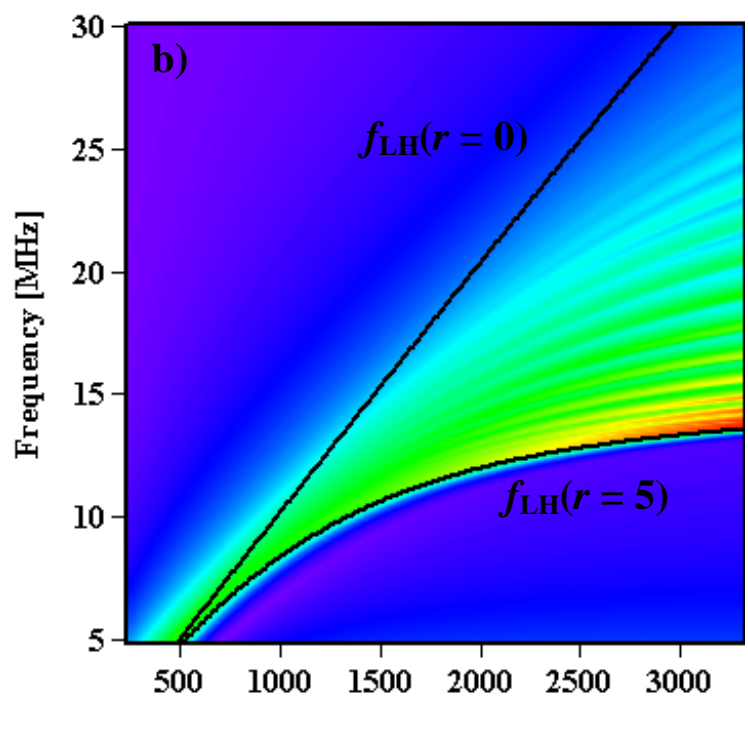

Magnetic Field [G]

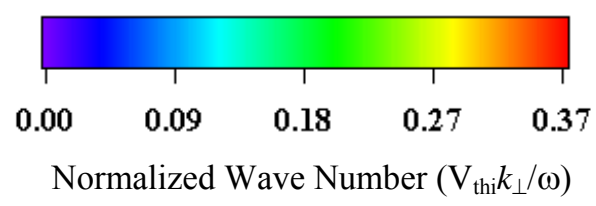

Figure 4.3 Normalized maximum values of the real component of $k_{\perp}$ as a function of rf driving frequency and magnetic field for a peak density of $2 \times 10^{13} \mathrm{~cm}^{-3}$, neutral pressure of $6.7 \mathrm{mTorr}$, electron temperature of $3 \mathrm{eV}$, ion temperature of $0.2 \mathrm{eV}$, and $k_{\|}$of $0.3 \mathrm{rad} / \mathrm{cm}$. a) Original model code. b) Revised model code with the black lines showing the calculated $f_{\mathrm{LH}}$ at the indicated radius.

The density profile used in the revised model, particularly the low densities at the plasma edge, is responsible for the principle differences between the original and revised model. The significant decrease in the density at the edge yielded wave numbers much larger than the original values as well as shifted the lower hybrid effects to much lower frequency. 
Another difference between the revised and original slow wave models are the visible banding in the figures. The bands in the plots of the normalized maximum and maximum wave numbers are a result of the discrete nature of the radial density profiles used in the models. To illustrate the banding more explicitly, the maximum wave number solutions (un-normalized) from Figures $4.2 \mathrm{~b}$ and $4.3 \mathrm{~b}$ are shown in Figure 4.4. The lower hybrid frequencies for densities at radii ranging from 0 to $5 \mathrm{~cm}$ are overlaid on the same figures. For each radial location, the wave numbers reach a local maxima near the lower hybrid frequency. When determining the maximum wave numbers through the entire radius, the local maxima for each radial step produces a band of large wave numbers near the lower hybrid resonance. Decreasing the radial density step size increases the number of possible resonance bands. Depending on the peak density, radial density profile, and resolutions in the rf frequency and magnetic field, the banding may be individually resolved or smeared out. The high density case (Figure 4.4a) indicates how the bands tend to be smeared out because the lower hybrid frequencies are much closer than those in the lower density case (Figure 4.4b). For the densities at radii of 0 and $1 \mathrm{~cm}$, the lower hybrid frequencies are very close to one another. Only for radii greater than $2 \mathrm{~cm}$ does the lower hybrid frequency begin to change significantly. At a radius of $5 \mathrm{~cm}$, where the density is the smallest, the wave numbers are the largest. 

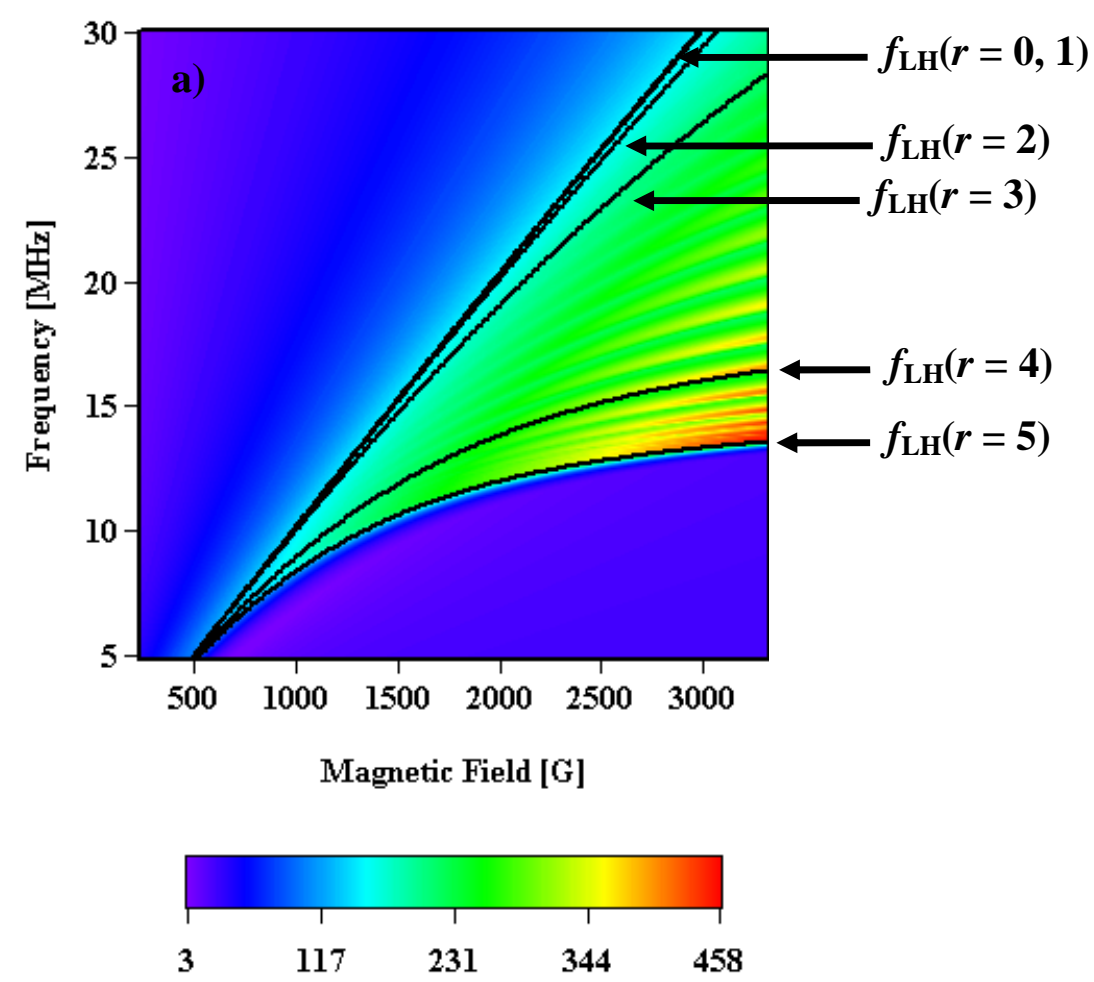

Maximum Wave Number $[\mathrm{rad} / \mathrm{cm}]$
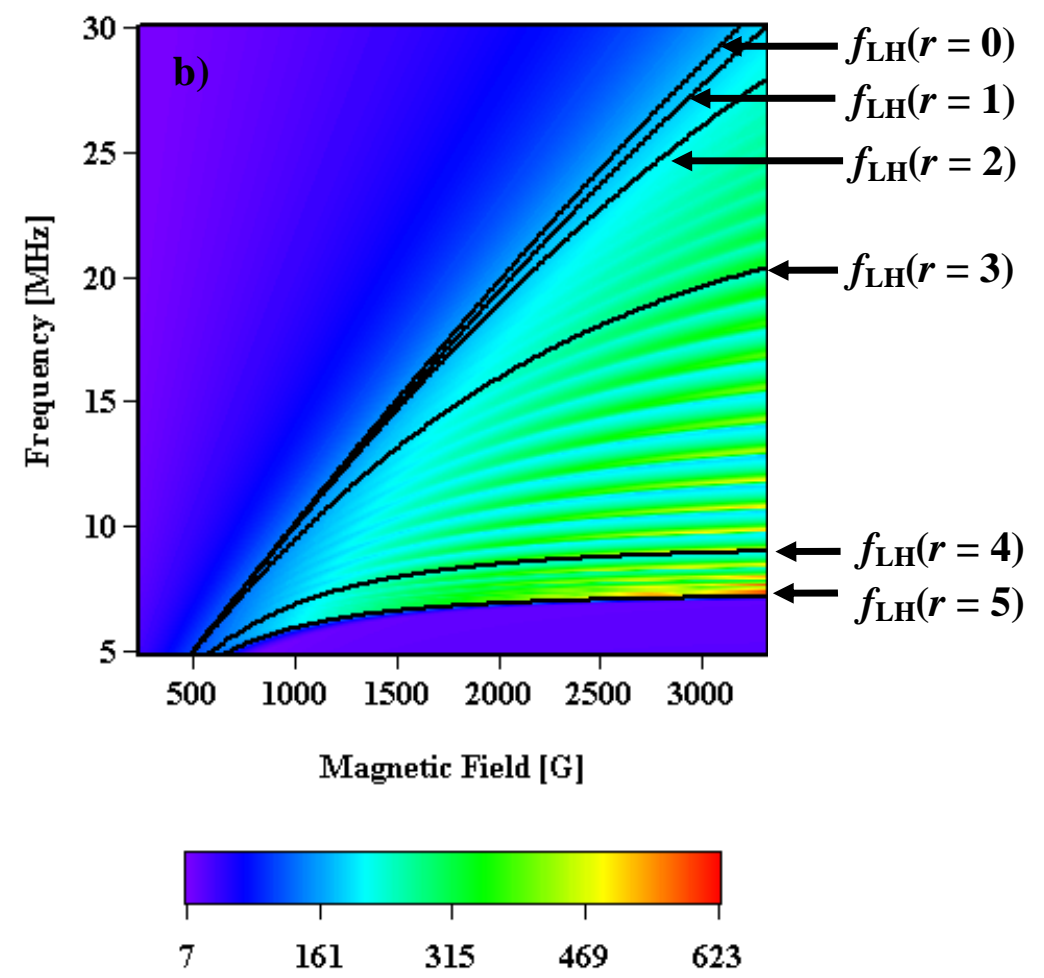

Maximum Wave Number [ $\mathrm{rad} / \mathrm{cm}]$

Figure 4.4 Maximum real $k_{\perp}$ as a function of rf driving frequency and magnetic field for peak densities of (a) $2 \times 10^{13} \mathrm{~cm}^{-3}$ and (b) $5 \times 10^{12} \mathrm{~cm}^{-3}$. The black lines show the calculated $f_{\mathrm{LH}}$ at the indicated radius. 
Thus, the original and revised models are consistent after accounting for the differences in the radial density profiles used, particularly at the edge. After narrowing the parameter space to the HELIX parameters, the revised model still predicts that the normalized wave numbers shown will be the largest at the plasma edge. The relevant regions of parameter spaces for HELIX from Figures $4.2 \mathrm{~b}$ and $4.3 \mathrm{~b}$ are shown in Figure 4.5 with the calculated on axis $(r=0)$ and edge $(r=5)$ lower hybrid frequencies for reference. The lower hybrid frequency at $r=5$ in $4.5 \mathrm{~b}$ is not shown because it is below 8 MHz. For these parameters, the largest normalized wave numbers fall between the axial and edge lower hybrid frequencies. Note that in this parameter range, the maximum values of the normalized wave numbers are relatively close. The important result here is that the largest normalized wave numbers, easily seen in Figure 4.5a, occur when the rf driving frequency is slightly larger than lower hybrid frequency at the edge. This indicates that the most likely location for ion heating is at the low density edge where the lower hybrid frequency is closest to the rf driving frequency. Comparing Figure $4.5 \mathrm{~b}$ with 1.7a indicates that the revised model still predicts that the same conditions shown to yield the largest perpendicular ion temperatures should have the largest slow wave, perpendicular wave numbers in the plasma edge. 

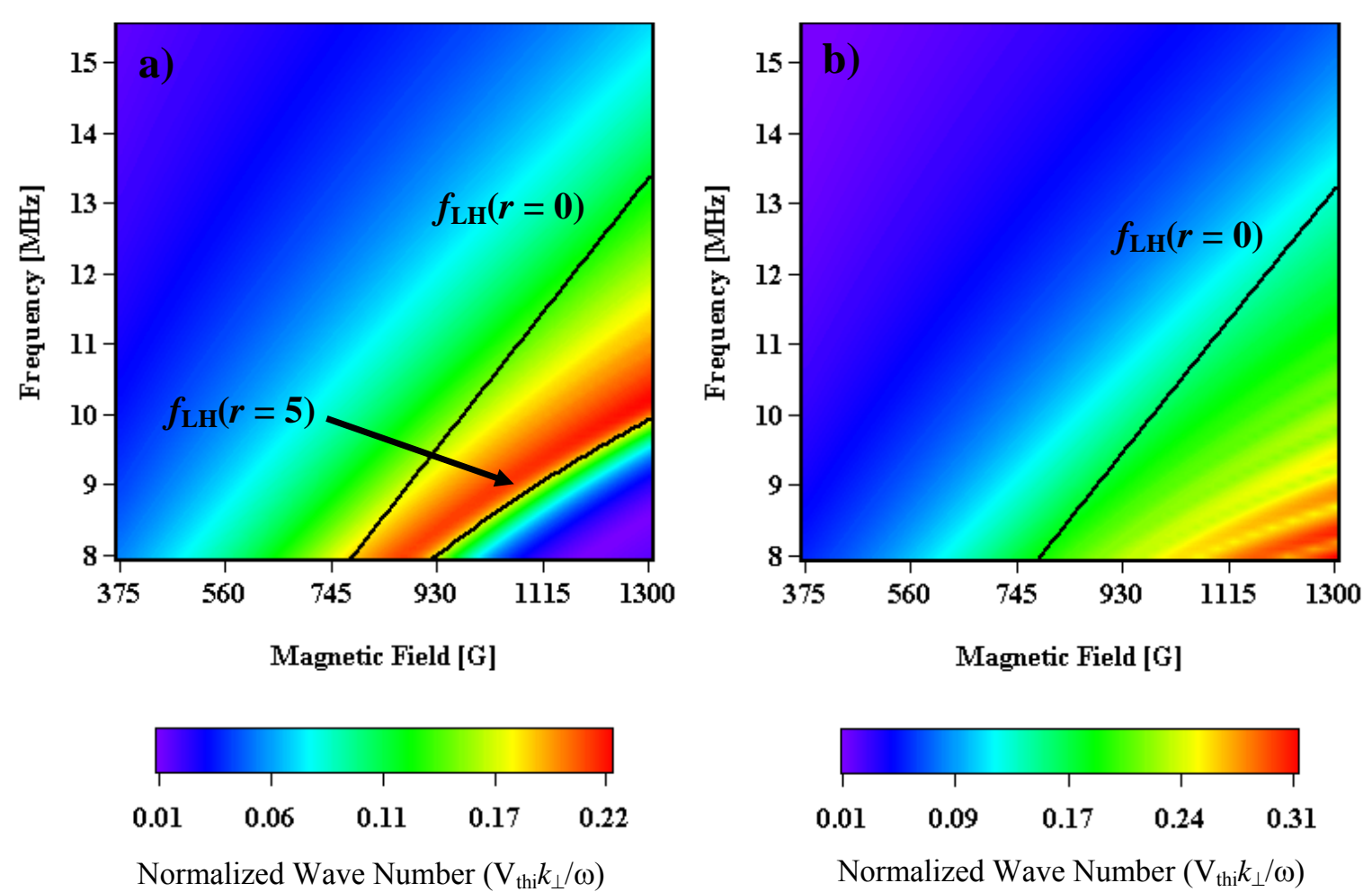

Normalized Wave Number $\left(\mathrm{V}_{\text {thi }} k_{\perp} / \omega\right)$

Figure 4.5 Normalized maximum values of the real component of $k_{\perp}$ for the operating parameters of HELIX as a function of rf driving frequency and magnetic field for peak densities of (a) $2 \times 10^{13} \mathrm{~cm}^{-3}$ and (b) $5 \times 10^{12} \mathrm{~cm}^{-3}$. The black lines show the calculated $f_{\mathrm{LH}}$ at the indicated radius.

\subsection{Model Predictions for the CTS Diagnostic}

One of the key issues identified in Kline's study of slow waves in helicon sources was that towards the edge of the discharge, the lower hybrid frequency decreases because of the role of the $\omega_{p i}$ term in the definition of $\omega_{L H}$. Therefore, the density used to calculate the expected slow wave perpendicular wave number is essentially a proxy for the radial location in the plasma. Based on the average of several radial density profiles measured with the Langmuir probe, a general form of the actual density profile in HELIX is given by

$$
N(r)=0.95 N_{0}\left(1-\left(\frac{r}{5.5}\right)^{2.2}\right)^{5}+0.05 N_{0}
$$


where $N_{0}$ is the peak density and $r$ is the radial location. The density profile is cut off at $5.5 \mathrm{~cm}$, where the edge density is assumed to be $5 \%$ of the peak density. A plot of the model radial density profile is shown in Figure 4.6 for a peak density of $2.25 \times 10^{13} \mathrm{~cm}^{-3}$.

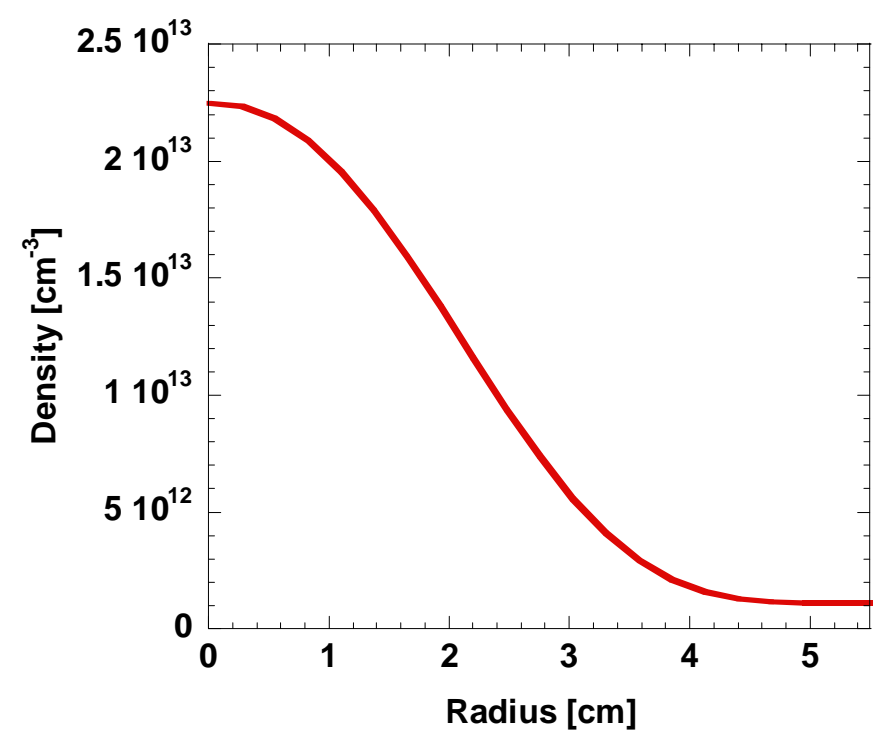

Figure 4.6 Radial density profile used for wave number predictions used in conjunction with the scattering diagnostic.

Since the intention is to detect the slow wave in HELIX with the CTS diagnostic, predicting the plasma parameters and radial location likely to result in measureable wave numbers will aid in the initial search. The current range of CTS measureable wave numbers is from 60 to $90 \mathrm{rad} / \mathrm{cm}$. For the typical range of HELIX $\mathrm{rf}$ frequencies and magnetic fields and a neutral pressure of 8 mTorr, Figure 4.7 shows the calculated wave numbers at radii of 0.5 to $5.5 \mathrm{~cm}$, in $1 \mathrm{~cm}$ increments. 

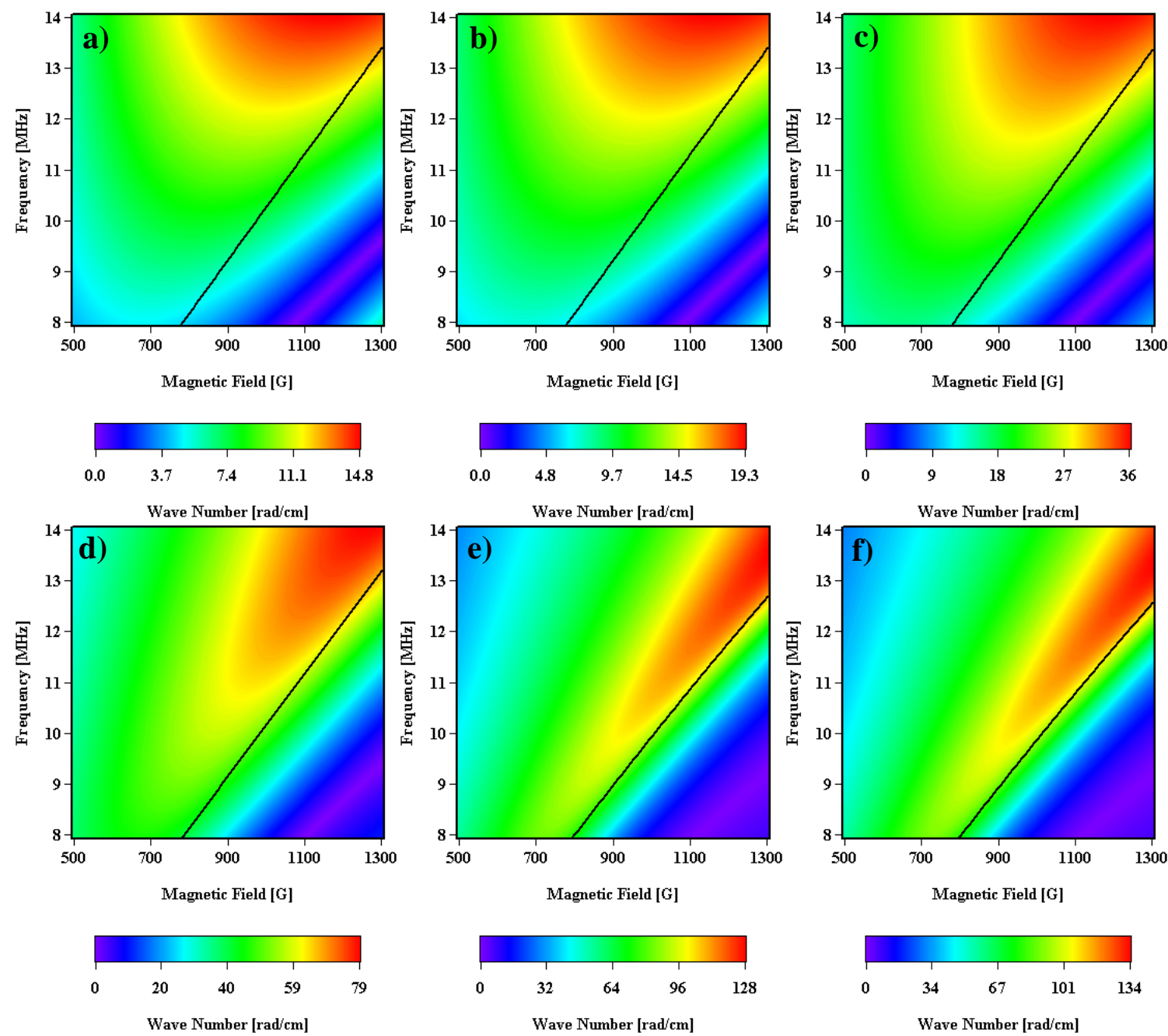

Figure 4.7 Values of the real component of $k_{\perp}$ as a function of rf driving frequency and magnetic field for a peak density of $2.25 \times 10^{13} \mathrm{~cm}^{-3}$, neutral pressure of $8 \mathrm{mTorr}$, electron temperature of $3 \mathrm{eV}$, ion temperature of $0.2 \mathrm{eV}$, and $k_{\|}$of $0.3 \mathrm{rad} / \mathrm{cm}$ at radii of (a) $0.5 \mathrm{~cm}$, (b) $1.5 \mathrm{~cm}$, (c) $2.5 \mathrm{~cm}$, (d) $3.5 \mathrm{~cm}$, (e) $4.5 \mathrm{~cm}$, (f) 5.5 $\mathrm{cm}$. The black line is the $f_{\mathrm{LH}}$ as calculated for each density at the given radius.

The black line in each plot of Figure 4.7 is the lower hybrid frequency for the density at that radial location. The lower hybrid frequency does not shift significantly for these parameters as the density decreases radially. Note the increase in the magnitude of the wave numbers and the correlation of the largest wave numbers with those frequencies just above the lower hybrid frequency. With the assumption that the largest wave numbers result in ion heating through Landau damping, demonstrating that the largest wave numbers are expected where the rf frequency is just above the local lower hybrid 
frequency provides important guidance for the design of the CTS system. These modeling results indicate that as the density decreases radially, the peak wave number increases to a maximum value of $134 \mathrm{rad} / \mathrm{cm}$ at a radius of $5.5 \mathrm{~cm}$. The region of largest wave numbers also narrows and shifts to lower frequencies towards the plasma edge.

The predicted perpendicular wave numbers are shown in Figure 4.8 as a function of radius and magnetic field for $\mathrm{rf}$ frequencies of $9.5,11.5$ and $13.5 \mathrm{MHz}$. In this presentation format, the radial location corresponding to each wave number value is easier to determine. As seen in the previous figures the largest perpendicular wave numbers occur in the edge where the density is the lowest.

Since the CTS system is designed to detect fluctuations at wave numbers ranging from 60 to $90 \mathrm{rad} / \mathrm{cm}$, those wave number ranges in Figure 4.8 are highlighted in Figure 4.9. The predicted CTS measureable wave number range is limited to radii greater than 3 $\mathrm{cm}$ and magnetic fields above 500 Gauss for an rf frequency of $9.5 \mathrm{MHz}$; above 700 Gauss for $11.5 \mathrm{MHz}$, and above 800 Gauss for $13.5 \mathrm{MHz}$. Note that for all rf frequencies, there is a window of radii and magnetic fields for which the CTS measurement range does not extend to large enough wave numbers. 

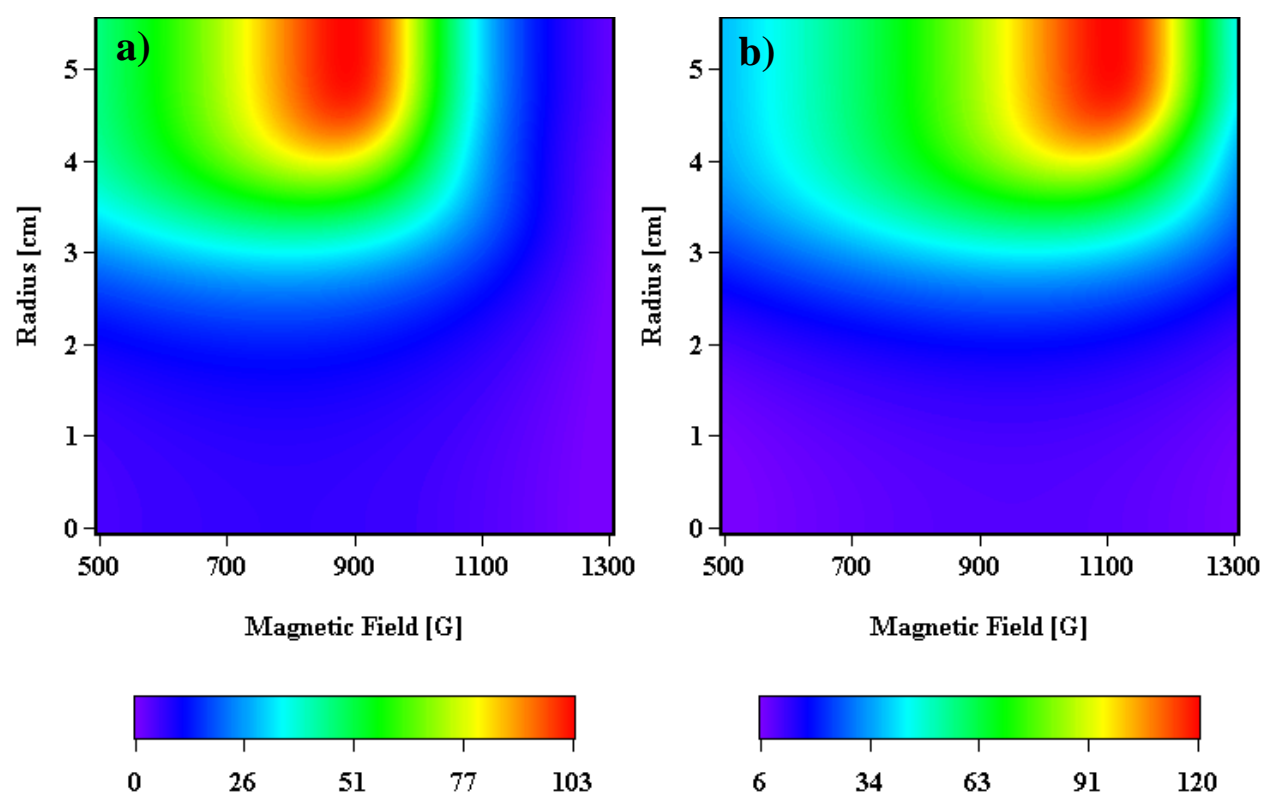

Wave Number [rad/cm]

Wave Number [ $\mathrm{rad} / \mathrm{cm}]$

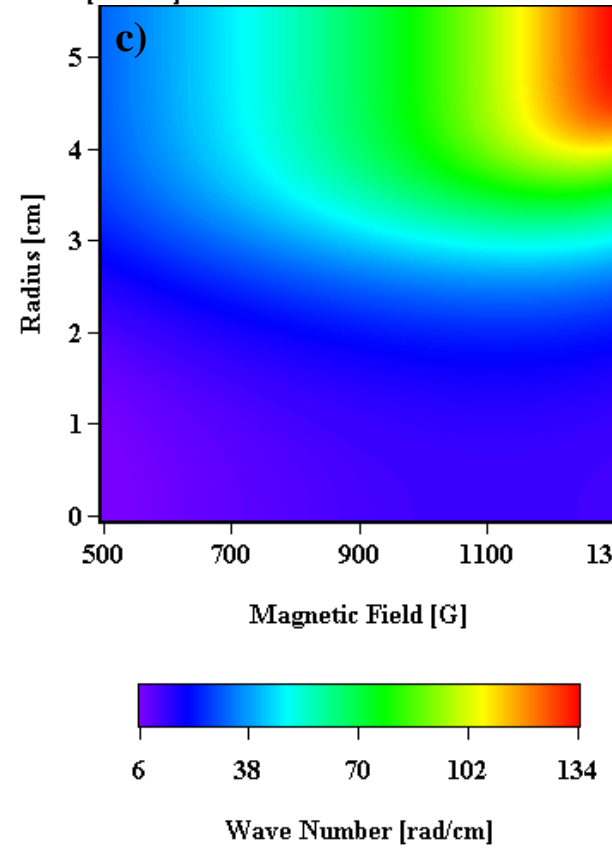

Figure 4.8 Values of the real component of $k_{\perp}$ as a function of radius and magnetic field for a peak density of $2.25 \times 10^{13} \mathrm{~cm}^{-3}$, neutral pressure of $8 \mathrm{mTorr}$, electron temperature of $3 \mathrm{eV}$, ion temperature of $0.2 \mathrm{eV}$, and $k_{\|}$of $0.3 \mathrm{rad} / \mathrm{cm}$ at $\mathrm{rf}$ driving frequencies of (a) is $9.5 \mathrm{MHz}$ (b) $11.5 \mathrm{MHz}$ and (c) is $13.5 \mathrm{MHz}$. 

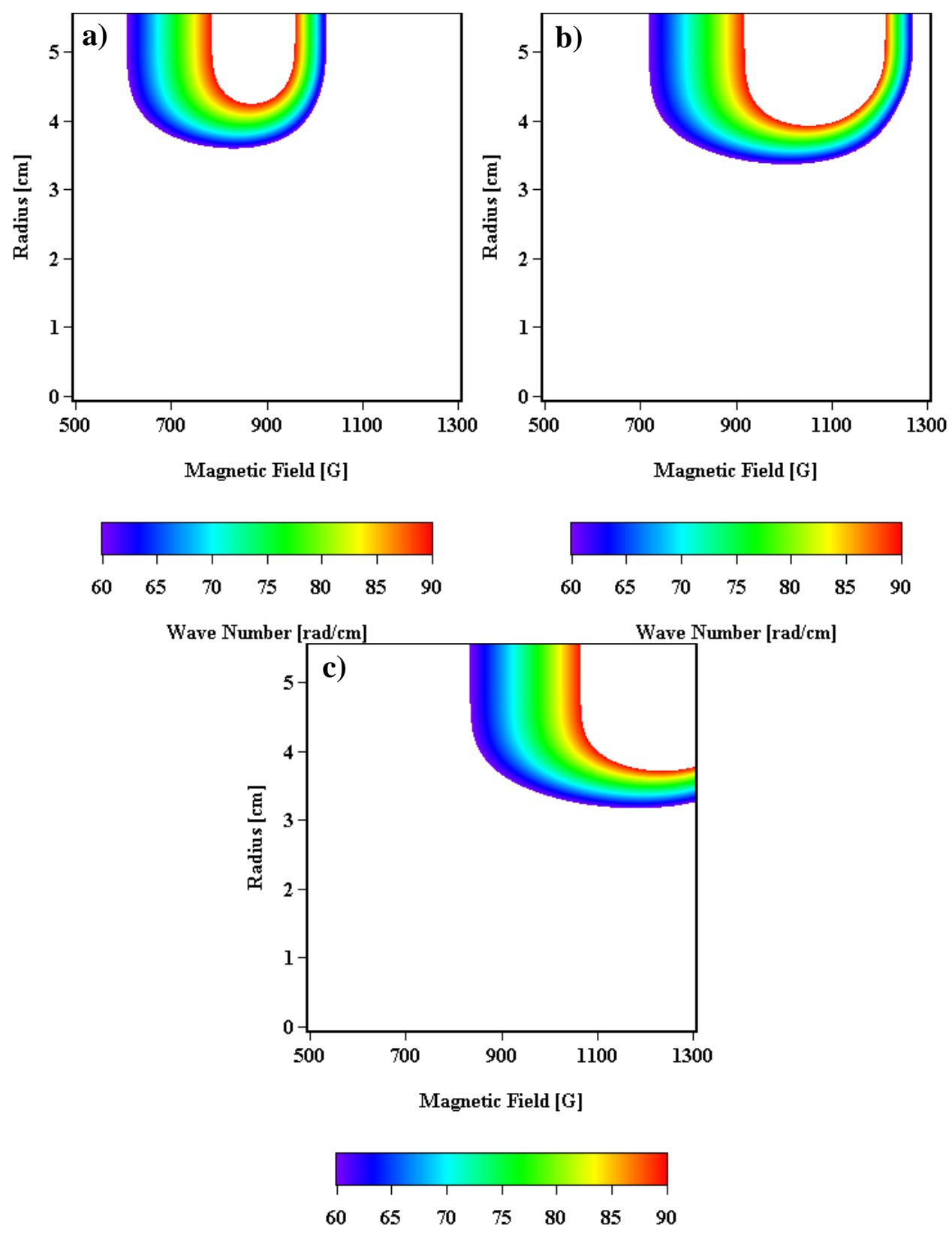

Wave Number [ $\mathrm{rad} / \mathrm{cm}]$

Figure 4.9 Scattering measureable range of wave number values from Figure 4.8. (a) is $9.5 \mathrm{MHz}$ (b) 11.5 $\mathrm{MHz}$ and (c) is $13.5 \mathrm{MHz}$. 


\section{Chapter 4 References}

${ }^{1}$ S. Cho, Phys. Plasmas 7, 417 (2000).

${ }^{2}$ M. Light, I.D. Sudit, F.F. Chen, Phys. Plasmas, 2, 4094 (1995).

${ }^{3}$ R.W. Boswell, Phys. Lett. A. 33, 457 (1970).

${ }^{4}$ A.W. Trivelpiece and R.W. Gould, J. Appl. Physics 17, 1784 (1959).

${ }^{5}$ F.F. Chen and D. Arnush, Phys. Plasmas 4, 3411 (1997).

${ }^{6}$ D.D. Blackwell, T.G. Madziwa, D. Arnush, F.F. Chen, Phys. Rev. Lett. 88, 145002 (2002).

${ }^{7}$ J.L. Kline, Slow Wave Ion Heating and Parametric Instabilities in the HELIX Helicon Source, Ph.D.

Dissertation, West Virginia University, Morgantown (2002). 


\section{Chapter 5: Collective Thomson Scattering}

Thomson scattering, in its most fundamental form, is simply the scattering of electromagnetic radiation from charged particles. When an incident monochromatic electromagnetic wave interacts with a charged particle, the particle will be accelerated in the oscillatory wave field, thereby emitting radiation. An illustration of the scattering from a single free particle for an incident monochromatic wave is shown in Figure 5.1.

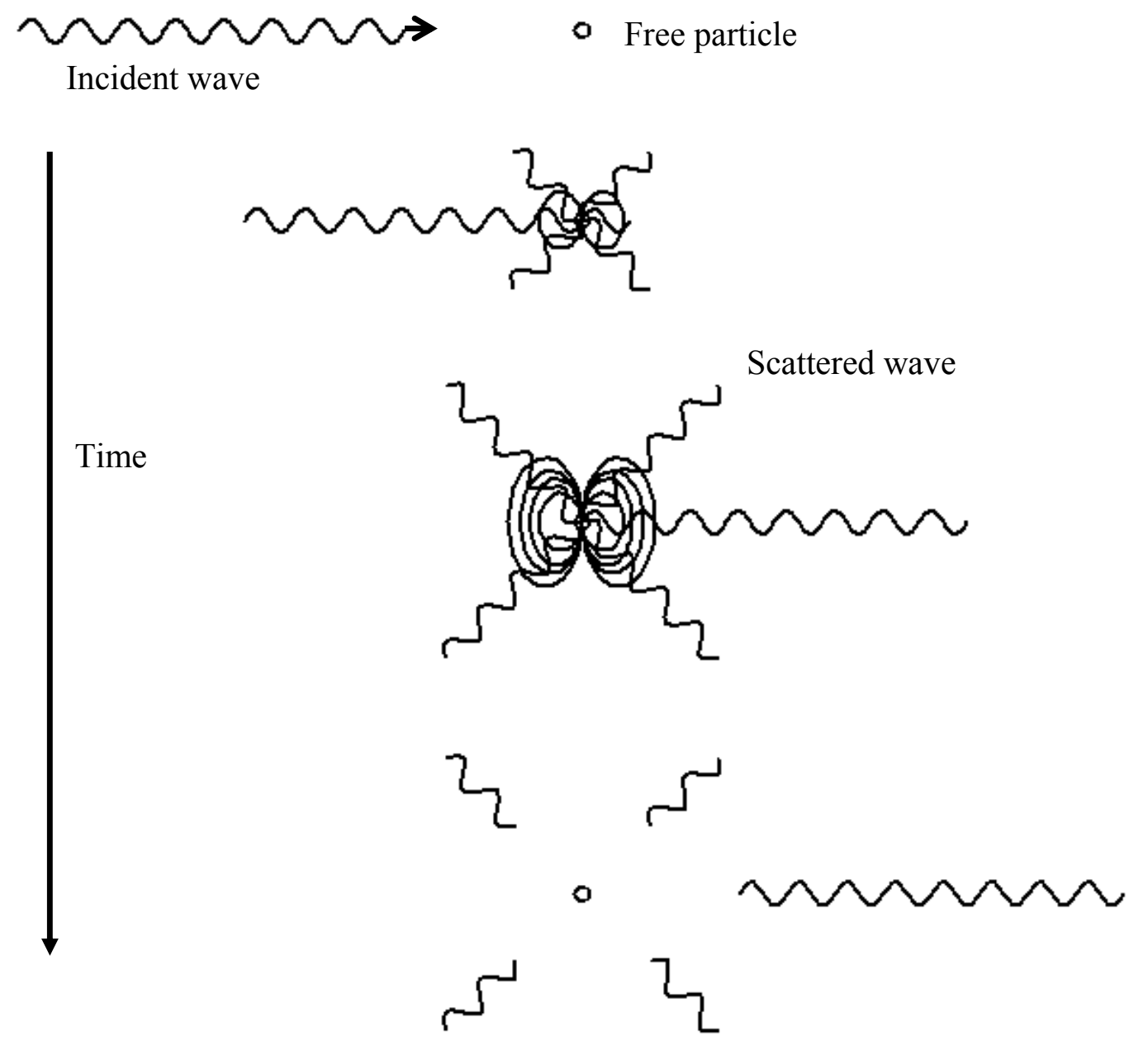

Figure 5.1 Thomson scattering from a free charge.

When deploying a Thomson scattering based diagnostic on a plasma experiement, the scattering ratio $k \lambda_{D e}$ is used to distinguish between coherent and incoherent scattering 
regimes; where $k$ is the wave number of the fluctuation and $\lambda_{D e}$ is the electron Debye length. The electron Debye length is

$$
\lambda_{D e}=743 \sqrt{\frac{T_{e}}{n_{e}}}[\mathrm{~cm}],
$$

where $T_{e}$ is the electron temperature in $\mathrm{eV}$ and $n_{e}$ is the electron density in $\mathrm{cm}^{-3}$. Substituting the Bragg condition, $k=2 k_{0} \sin \left(\theta_{s} / 2\right)$, into the dimensionless ratio and replacing the incident wave number with the incident wavelength, $k_{0}=2 \pi / \lambda_{0}$, the ratio can be written in the form

$$
k \lambda_{D e}=\frac{4 \pi}{\lambda_{0}} \sin \left(\theta_{s} / 2\right) \lambda_{D e} .
$$

In this form, the dependence on the incident wavelength $\left(\lambda_{0}\right)$, scattered angle $\left(\theta_{s}\right)$, and electron Debye length $\left(\lambda_{D e}\right)$ of the scattering ratio is explicitly shown.

When the ratio is larger than unity

$$
\frac{4 \pi}{\lambda_{0}} \sin \left(\theta_{s} / 2\right) \lambda_{\text {De }} \geq 1
$$

the scattering is incoherent. The maximum incident wavelength for incoherent scattering (at $\theta_{s}=\pi$ ) is given by

$$
4 \pi \lambda_{\text {De }} \geq \lambda_{0},
$$

i.e. the incident wavelength must be smaller than roughly ten times the Debye length. A simple one dimensional illustration of incoherent scattering is shown in Figure 5.2a. When the incident wavelength is shorter than the Debye length, individual electrons inside a Debye sphere are accelerated independently by the incident wave field. Since the electrons are accelerated in multiple directions and by multiple amounts, the 
corresponding scattered radiation is random in phase as well; hence the incoherent scattering description. For a fixed scattering geometry, the frequency spectrum for incoherent scattering is directly proportional to the one dimensional electron velocity distribution function along $k$. This is a result of the individual electrons in the velocity distribution function along $k$, producing a Doppler shifted signal relative to the incident frequency.

a)
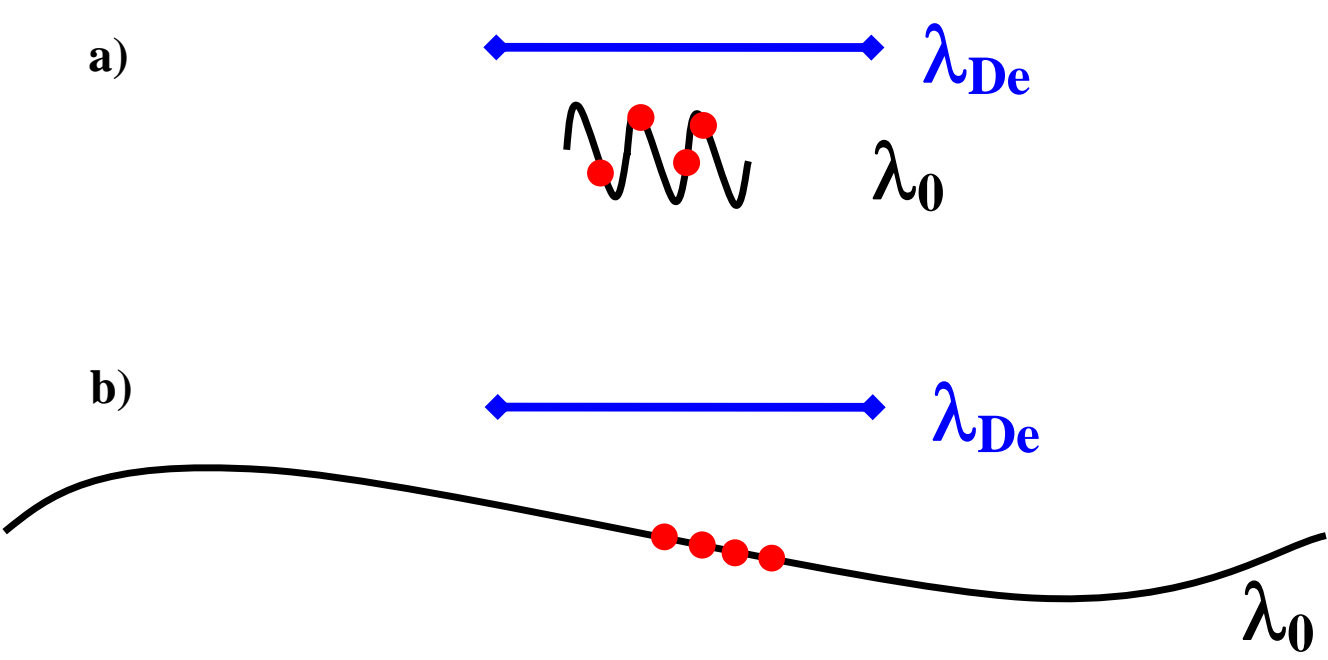

Figure 5.2 One dimensional description of the (a) incoherent and (b) coherent scattering conditions showing the relative sizes of the incident wavelength $\left(\lambda_{0}\right)$ and the Debye length $\left(\lambda_{\text {De }}\right)$. The $(\bullet)$ represent electrons and how they are accelerated relative to one another depends on the size of the incident wavelength.

When the scattering ratio condition is reversed

$$
4 \pi \lambda_{\text {De }} \leq \lambda_{0},
$$

coherent (collective) scattering is obtained. The descriptors "coherent" and "collective" tend to be interchanged frequently in the literature even though there are subtle but distinct differences between the two types of scattering. The term "collective" is the more appropriate descriptor for the $300 \mathrm{GHz}$ diagnostic described here. The collective nature of collective Thomson scattering arises because with the incident wavelength larger than 
the Debye length (see Figure 5.2b), all the electrons inside a Debye sphere are similarly accelerated in the incident wave field, producing a collective scattering signal. It is in the collective regime that plasma waves such as electron plasma waves, ${ }^{1}$ ion acoustic waves, ${ }^{2,3}$ ion Bernstein waves, ${ }^{4}$ lower hybrid waves, ${ }^{5}$ and even turbulence ${ }^{6,7}$ have been measured with laser and microwave scattering diagnostics.

The essential steps in the calculation of the power scattered in a CTS experiment are reviewed here to highlight the general principles involved in making a collective scattering measurement. The reader is referred to several excellent reviews in the literature for a full treatment. ${ }^{8,9,10,11}$ For collective scattering we must calculate the sum of the scattered electric fields from all the contributing electrons located in the scattering volume. Assuming the scattering is produced from a monochromatic incident radiation

$$
\vec{E}_{i}(\vec{r}, t)=\vec{E}_{i} \exp \left(i\left(k_{i} r-\omega_{i} t\right)\right)
$$

with a constant amplitude $E_{i}$ across the beam diameter and along the scattering volume, the resulting scattered power spectrum, after applying Parseval's theorem is

$$
\frac{d^{2} P}{d \nu_{s} d \Omega_{s}}=\frac{\varepsilon_{0} c x^{2}}{2 T}\left|E_{s}\left(v_{s}\right)+E_{s}^{*}\left(-v_{s}\right)\right|^{2}
$$

where the scattered electric field $\left(E_{s}\right)$, in the dipole approximation, is defined as

$$
E_{s}\left(v_{s}\right)=\frac{r_{e} \exp (\vec{k} \cdot \vec{x})}{2 \pi x} \vec{\Pi} \cdot \vec{E}_{i} \int_{T} \int_{V} n_{e} \exp (-i(\vec{k} \cdot \vec{r}-\omega t)) d t d^{3} r
$$

with $\vec{\Pi} \cdot \vec{E}_{i}=\left|\hat{k}_{s} \times\left(\hat{k}_{s} \times \vec{E}_{i}\right)\right|=\sin (\varphi) \vec{E}_{i}$ and the angle $\varphi$ is the angle between the electric field polarization of the incident radiation and the direction of the scattered radiation (See 
Figure 5.3a). Substituting Equation 5.8 into 5.7 and taking $\varphi=90^{\circ}$ results in the scattered power spectrum

$$
\frac{d^{2} P}{d v_{s} d \Omega_{s}}=\frac{r_{e}^{2} P_{i}}{A} n_{e} V S(\vec{k}, \omega)
$$

where $P_{i}$ is the total incident power across the scattering volume $\left(P_{i}=A / 2 \varepsilon_{0} c\left|E_{i}\right|^{2}\right)$, and $S(\vec{k}, \omega)$ is the scattering form factor. Neglecting the higher frequency term, the scattering form factor is defined as

$$
S(\vec{k}, \omega)=\frac{1}{n_{e} T V}\left|n_{e}(\vec{k}, \omega)\right|^{2}
$$

where $n_{e}(\vec{k}, \omega)$ is the Fourier transform of the density fluctuation. Taking the density fluctuation to be $\tilde{n}_{e} \cos (k x-\omega t)$ with the wave propagating perpendicular to the incident laser, we can integrate Equation 5.9 over the scattered frequency $\left(v_{s}\right)$ and the scattering collection angle $\left(\Omega_{s}\right)$. The resulting scattered power is given by

$$
P_{s}=\frac{1}{4} P_{i} r_{e}^{2} \lambda_{0}^{2} L_{v}^{2}(\tilde{n})^{2}
$$

where $P_{i}$ is the incident beam power, $r_{e}$ is the classical electron radius, $\lambda_{0}$ is the incident wavelength, $L_{v}$ is the length of the scattering volume, and $\tilde{n}$ is the density fluctuation amplitude. An illustration of the scattering volume length $\left(L_{v}\right)$ is shown in Figure 5.3b. A key feature of Equation 5.11 is that the scattered power is a function of the square of the incident wavelength. Assuming comparable scattering volume lengths and density fluctuation amplitudes, increasing the incident wavelength from $10.6 \mu \mathrm{m}\left(\mathrm{CO}_{2}\right.$ laser $)$ to 1 $\mathrm{mm}(300 \mathrm{GHz})$ reduces the amount of incident power required to produce the same 
amount of scattered power by a factor of $\sim 10,000$. In terms of personnel safety, this difference means that, for detection sensitivities being equal, a $10 \mathrm{~mW}$ microwave source provides the equivalent scattered power of a $100 \mathrm{~W} \mathrm{CO}_{2}$ laser.

a)

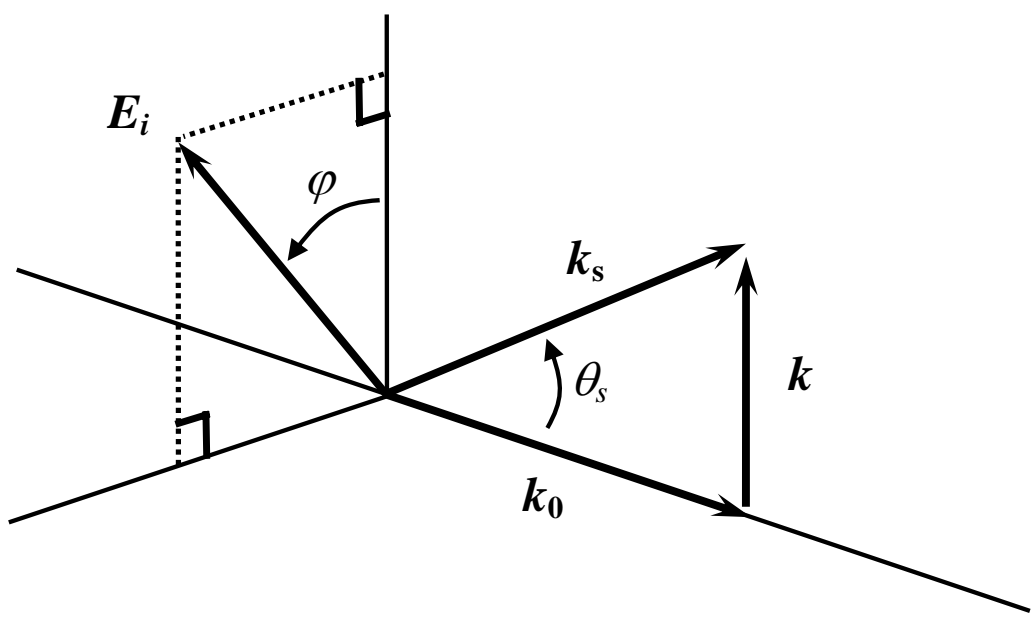

b)

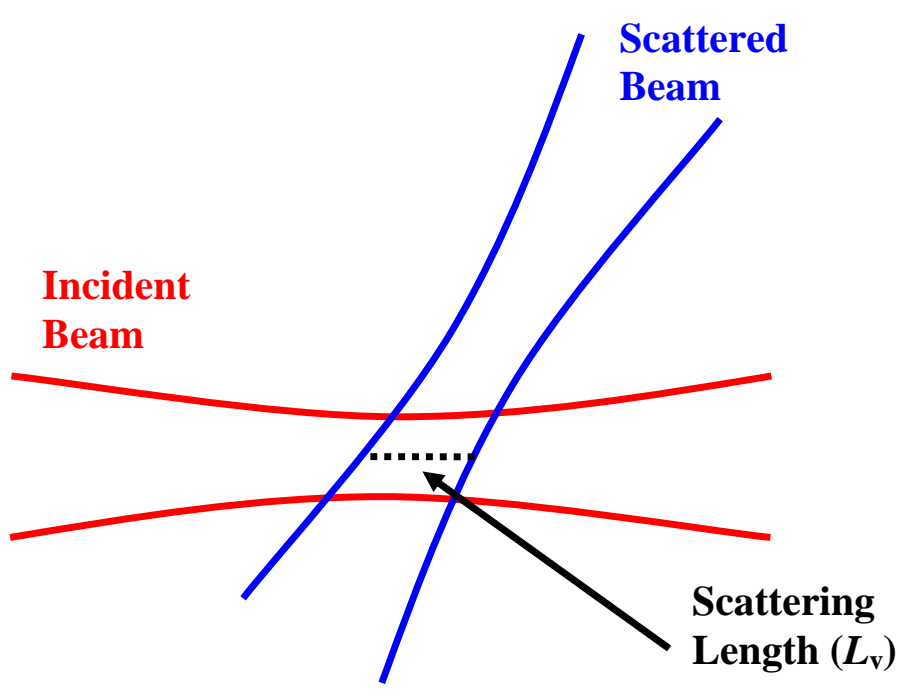

Figure 5.3 (a) Scattering geometry and definition of parameters. (b) Finite scattering volume and length for the general scattering geometry.

When using a collective scattering diagnostic to measure fluctuations in plasma, there are several elements to consider: The incident wavelength and power, the expected range of fluctuation wavelengths (wave numbers), the available optical access for the incident and scattered radiation, and the detection method. The first three are interrelated 
through the scattered power relation and the Bragg condition $\left(k=2 k_{0} \sin \left(\theta_{s} / 2\right)\right)$. The available optical access for the incident and scattered radiation determines the measureable range of wave numbers based on the incident wave number and the accessible collection angles for the scattered radiation. The chosen incident wavelength and the available power in the laser, for a given density fluctuation amplitude, determines the amount of scattered radiation available for detection.

In general there are three ways to measure a Thomson scattered signal. For systems that use an incident wavelength in the visible or near visible range, a spectrometer may be used for detection. When using a spectrometer, the specific grating will determine the spectral resolution available in a scattered spectrum measurement. The spectral shift, relative to the incident frequency, is used to determine the fluctuation frequency, while the scattering geometry determines the wave number of the measured fluctuation. With the frequency and wave number of the fluctuation measured, the wave characteristics (phase speed, etc.) and dispersion relations may be obtained.

When the incident wavelength for a CTS diagnostic is in the far infrared (FIR) or microwave range, a solid state detector such as a Schottky diode is typically used to measure the scattered radiation. These types of detectors have a nonlinear current response of the form

$$
I \propto V+V^{2}
$$

where $I$ is the output current and $V$ is the voltage (as driven by the laser radiation). If the diode is simultaneously driven by two sinusoidal frequency sources, a scattered source $\left(E_{\text {sig }} \cos \left(\omega_{\text {sig }} t\right)\right)$ and a local oscillator source $\left(E_{L O} \cos \left(\omega_{L O} t\right)\right)$ the output current of the diode will be 


$$
I \propto E_{\text {sig }} \cos \left(\omega_{\text {sig }} t\right)+E_{L O} \cos \left(\omega_{L O} t\right)+\left(E_{\text {sig }} \cos \left(\omega_{\text {sig }} t\right)+E_{L O} \cos \left(\omega_{L O} t\right)\right)^{2}
$$

where $E_{\text {sig }}$ and $E_{L O}$ are the respective electric field amplitudes and $\omega_{\text {sig }}$ and $\omega_{L O}$ are the frequencies of the corresponding signals. Neglecting the linear response of the diode, the resulting expression of the detector response becomes

$$
\begin{aligned}
I \propto \frac{E_{s i g}^{2}+E_{L O}^{2}}{2}+\frac{E_{s i g}^{2}}{2} \cos \left(2 \omega_{s i g} t\right)+\frac{E_{L O}^{2}}{2} \cos \left(2 \omega_{L O} t\right)+ \\
E_{s i g} E_{L O} \cos \left(\left(\omega_{s i g}+\omega_{L O}\right) t\right)+E_{s i g} E_{L O} \cos \left(\left(\omega_{s i g}-\omega_{L O}\right) t\right) .
\end{aligned}
$$

The first term of Equation 5.14 is a constant DC offset that is generally neglected and eliminated by filtering. The second, third, and fourth terms are the high frequency components, and they too are typically eliminated by filtering. The last term, $E_{\text {sig }} E_{L O} \cos \left(\left(\omega_{\text {sig }}-\omega_{L O}\right) t\right)$, produces the difference frequency $\left(\Delta f=\omega_{\text {sig }}-\omega_{L O}\right)$ typically referred to as the intermediate frequency (IF). The IF is the basis for the common description of diode-based detectors as being detector-mixers. The nonlinear response of the diode leads to frequency mixing of the incident signals and is the feature that provides the basis of distinction between homodyne and heterodyne detection schemes.

Homodyne detection employs a single frequency source for both the interaction and local oscillator beams. Like an interferometer, a portion of the source beam is used as a local oscillator source. The remaining portion of the source beam is used for interacting with the plasma. A diagram illustrating the similarity between an interferometer and a simple CTS diagnostic employing homodyne detection is shown in Figure 5.4. In essence, an interferometer is a zero angle, collective scattering diagnostic. Unlike the interferometer, the scattered signal from CTS will be Doppler shifted relative to the 
original frequency. When the local oscillator and Doppler shifted scattered signals are both incident upon the detector-mixer, the resultant IF is measured.
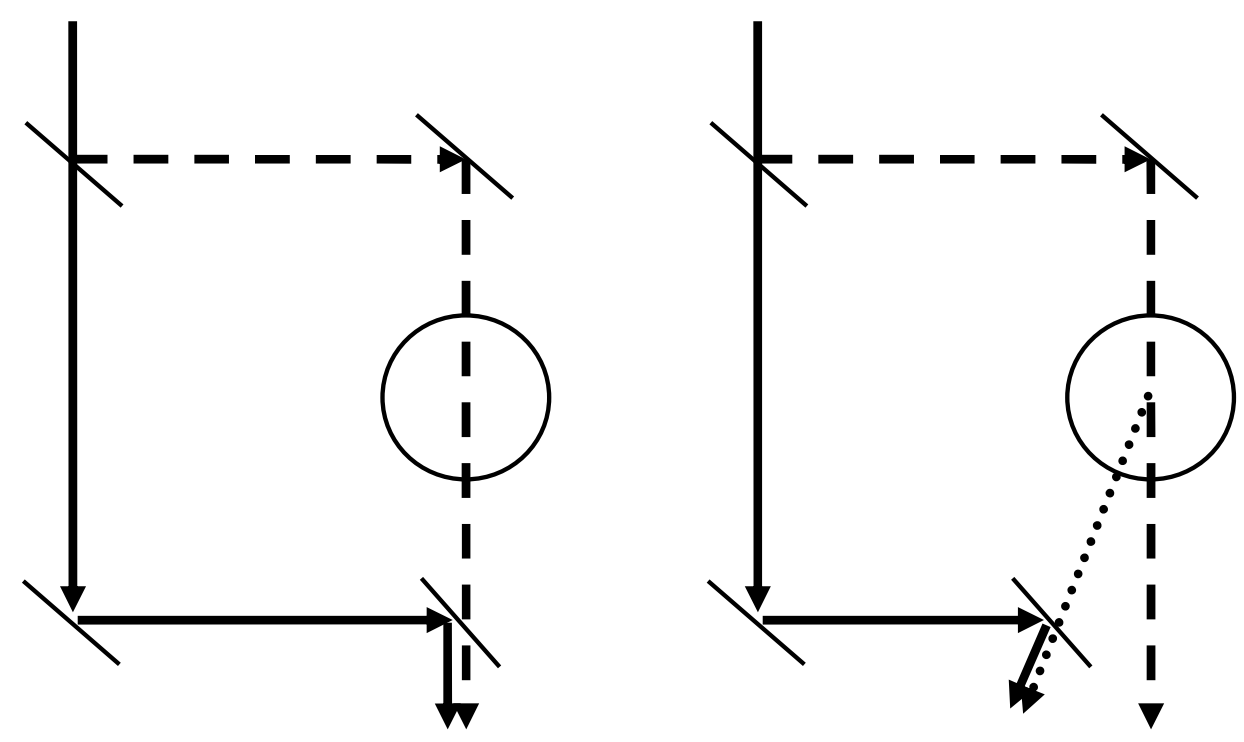

Figure 5.4 Similarity between an (a) interferometer and (b) a simple CTS diagnostic employing a homodyne detection scheme.

Heterodyne detection employs a local oscillator with a frequency different from that of the of the interaction beam. A second laser or a mechanical process can be used to produce a local oscillator frequency different from the frequency used for the interaction beam. Two methods used to mechanically introduce a frequency shifted local oscillator from a portion of the original source are an acoustic optical modulator ${ }^{12}$ and a rotating diffraction grating. ${ }^{13}$ For a more extensive discussion on the detection methods and their respective properties see Ref. 14.

The fundamental difference between the homodyne and heterodyne detection methods is the ability to differentiate the wave direction based on the relative frequency shift. For a fixed scattering geometry, homodyne detection cannot differentiate between a 
signal that has been Doppler shifted up or down in frequency. To illustrate the difference, an example is provided, with the following definitions:

Incident frequency $\omega_{0} / 2 \pi=300 \mathrm{~Hz}$

Wave frequency $\omega_{w} / 2 \pi=50 \mathrm{~Hz}$

Homodyne local oscillator frequency $\omega_{L O 1} / 2 \pi=300 \mathrm{~Hz}$

Heterodyne local oscillator frequency $\omega_{\mathrm{LO} 2} / 2 \pi=200 \mathrm{~Hz}$

Positive Doppler shifted frequency $\omega_{\text {sig+ }} / 2 \pi=\left(\omega_{0}+\omega_{w}\right) / 2 \pi=350 \mathrm{~Hz}$

Negative Doppler shifted frequency $\omega_{\text {sig- }} / 2 \pi=\left(\omega_{0}-\omega_{w}\right) / 2 \pi=250 \mathrm{~Hz}$

The resultant frequency spectra for the homodyne and heterodyne methods are calculated by substituting the appropriate frequencies as defined into Equation 5.14 are shown in Figure $5.5 \mathrm{a}$ and $5.5 \mathrm{~b}$. The important features for comparison are the low frequency spectra $(f<200 \mathrm{~Hz})$. In this example, the higher frequencies are relatively close to the low frequency difference frequencies. For realistic experimental frequencies on the order of a few $\mathrm{GHz}$ to $\mathrm{THz}$, the higher frequency components would generally be larger than the detector's bandwidth. The homodyne spectra shows how mixing both the positive and negative Doppler shifted frequencies with a local oscillator at the same frequency as the incident frequency results in the same difference frequency. Conversely, the difference frequencies in the heterodyne spectra by using a local oscillator different from the incident frequency are offset from one another, allowing for determination of the wave direction. 

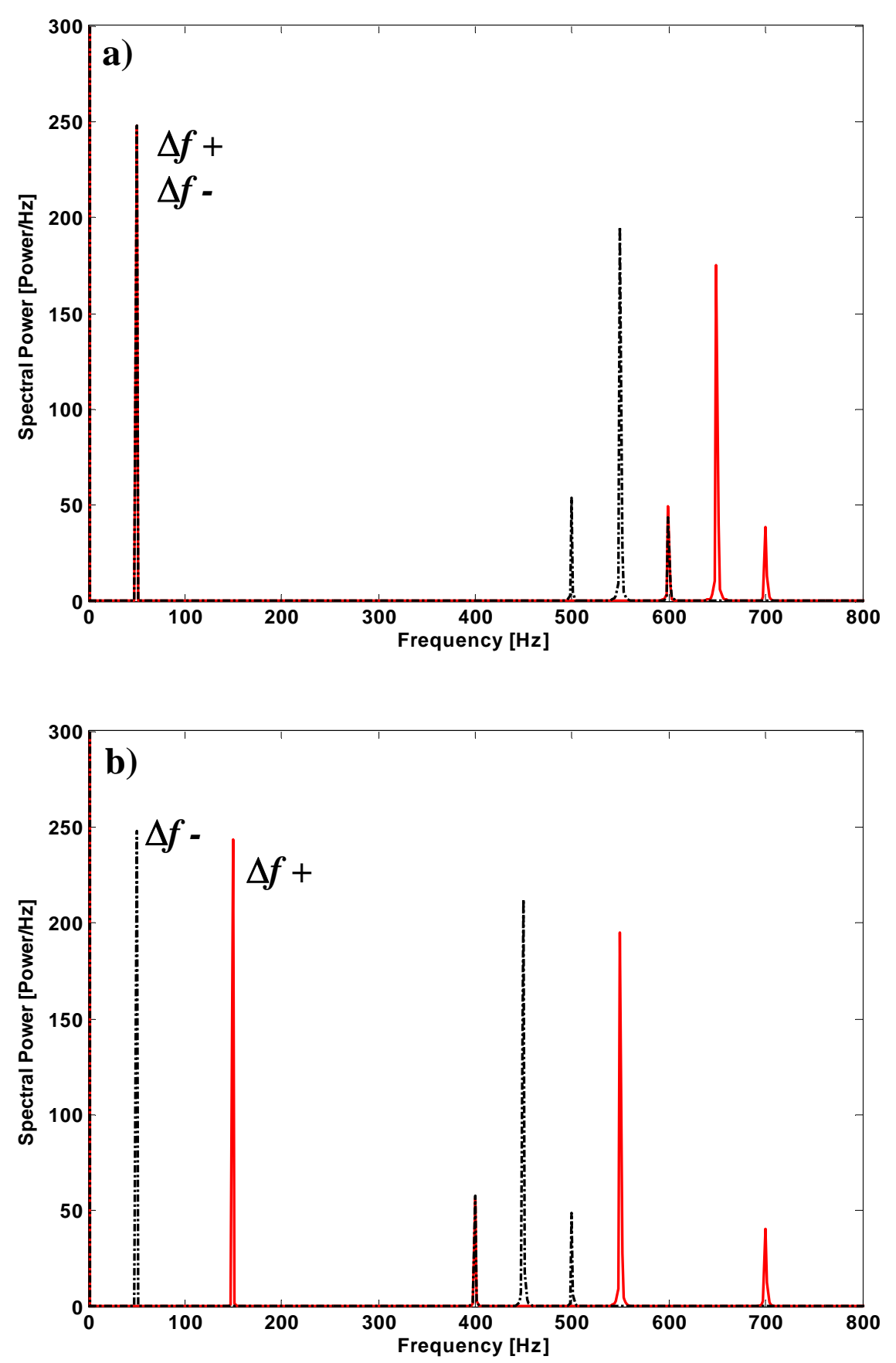

Figure 5.5 Simulated frequency spectra of a (a) homodyne and (b) heterodyne detection scheme. $\Delta f+$ and $\Delta f$ - are the positive and negative Doppler shifted mixer output frequencies with the rest of the higher frequency terms in Equation 5.14. 


\section{Chapter 5 References}

${ }^{1}$ K. Dzierzega, W. Zawadzki, B. Pokrzywka, and S. Pellerin, Phys. Rev. E, 74, 026404 (2006).

${ }^{2}$ A.L. Peratt, R.L. Watterson, and H. Derfler, Phys. Fluids, 20, 1900 (1977).

${ }^{3}$ F. Pisani, T. Pierre, and D. Batani, J. Plasma Phys., 59, 69 (1998).

${ }^{4}$ Y. Takase, J.D. Moody, C.L. Fiore, F.S. McDermott, M. Porkolab, and J. Squire, Phys. Rev. Lett., 59, 1201 (1987).

${ }^{5}$ G.A. Wurden, K.L. Wong, and M. Ono, Phys. Fluids, 28, 716 (1985).

${ }^{6}$ D.L. Brower, W.A. Peebles, S.K. Kim, and N.C. Luhmann Jr., Rev. Sci. Instrum., 59, 1559 (1988).

${ }^{7}$ T.L. Rhodes, W.A. Peebles, X. Nguyen, M.A. VanZeeland, J.S. deGrassie, E.J. Doyle, G. Wang, and L. Zeng, Rev. Sci. Instrum., 77, 10E922 (2006).

${ }^{8}$ I.H. Hutchinson, Principles of Plasma Diagnostics, (Cambridge University Press, Cambridge, 1987).

${ }^{9}$ R.E. Slusher and C.M. Surko, Phys. Fluids, 23, 472 (1980).

${ }^{10}$ J. Sheffield, Plasma Scattering of Electromagnetic Radiation, (Academic, New York, 1975).

${ }^{11}$ D.E. Evans and J. Katzenstein, Rep. Prog. Phys., 32, 207 (1969).

${ }^{12}$ C.M. Surko, R.E. Slusher, D.R. Moler, and M. Porkolab, Phys. Rev. Lett., 29, 81 (1972).

${ }^{13}$ H. Park, D.L Brower, W.A. Peebles, N.C. Luhmann Jr., R.L. Savage Jr., and C.X. Yu, Rev. Sci. Instrum., 56, 1055 (1985).

${ }^{14}$ R.E. Slusher and C.M. Surko, Phys. Fluids, 23, 472 (1980). 


\section{Chapter 6: 300 GHz Scattering Diagnostic Development}

As noted in Chapter 5, there are distinct advantages, in terms of scattered power amplitude and safety, to performing CTS in the microwave regime. Since radiation at 300 $\mathrm{GHz}$ resides on the cusp between the microwave and far infrared ranges, transmission is accomplished by either standard waveguide or quasioptical propagation. Two drawbacks to using waveguides at $300 \mathrm{GHz}$ are the size and expense of the waveguide. The waveguide used for the transmission of $300 \mathrm{GHz}$ is WR-3, which has internal dimensions of $0.0340 " \mathrm{x} 0.0170 " .{ }^{1}$ The precision needed to produce this size of waveguide with minimal defects along the internal surfaces makes using waveguide a very costly option. Additionally, any defects on the internal surfaces cause losses and also produce higher order modes, sapping power from the fundamental mode.

Another drawback to using waveguide arises with the insertion of additional components in the waveguide, such as tees for splitting or recombining the radiation. The problem lies in the alignment of the inserted waveguide components. Slight misalignments of the components can cause severe loss in the transmitted power. An excellent discussion on the subject using WR-10 waveguide is given by Kerr. ${ }^{2}$

Because of the cost and other problems associated with waveguide propagation, quasioptical propagation was the method chosen for the $300 \mathrm{GHz}$ CTS system. In quasioptical beam propagation, more conventional components such as lenses and mirrors are used for manipulating the beam and beam path. A discussion of the theory for quasioptical beam propagation is given in Section 6.1 , followed by the initial system design using ZEMAX in Section 6.2. A discussion of the testing and installation of the 
components is contained in Section 6.3, followed by a discussion of the proof-of-concept test using a rotating diffraction grating in Section 6.4.

\subsection{Quasioptical Gaussian Beam Propagation Theory}

In this section, the equations governing the propagation of the fundamental quasioptical Gaussian beam are derived from first principles. This derivation generally follows the procedure outlined in Goldsmith. ${ }^{3}$ The fundamental importance of the Gaussian beam parameters that are derived here is that they provide a structure consisting of only a few simple functions that define the entire beam and associated propagation characteristics. Having a simple collection of beam parameter functions allow programs like ZEMAX, an optical design code, to assist in developing optical systems employing quasioptical Gaussian beam propagation.

Since the Gaussian beam will generally be propagating in free space (air or vacuum), we start with Maxwell's equations in vacuum:

$$
\begin{aligned}
& \vec{\nabla} \cdot \vec{E}=0 \\
& \vec{\nabla} \times \vec{E}=-\frac{\partial \vec{B}}{\partial t} \\
& \vec{\nabla} \cdot \vec{B}=0 \\
& \vec{\nabla} \times \vec{B}=\mu_{0} \varepsilon_{0} \frac{\partial \vec{E}}{\partial t}
\end{aligned}
$$

Following the typical process of finding the wave equation, we take the curl of Equation (6.2) $\vec{\nabla} \times(\vec{\nabla} \times \vec{E}=-\partial \vec{B} / \partial t)$, which leads to $\vec{\nabla}(\vec{\nabla} \cdot \vec{E})-\nabla^{2} \vec{E}=-\partial / \partial t(\vec{\nabla} \times \vec{B})$. Applying the condition that the divergence of the electric field is zero in free space, we 
have $\nabla^{2} \vec{E}=\partial / \partial t(\vec{\nabla} \times \vec{B})$. Substitution of Equation (6.4) results in $\nabla^{2} \vec{E}=\partial / \partial t\left(\mu_{0} \varepsilon_{0} \partial \vec{E} / \partial t\right)$ and the general equation for free space wave propagation is obtained,

$$
\nabla^{2} \vec{E}=\mu_{0} \varepsilon_{0} \frac{\partial^{2}}{\partial t^{2}} \vec{E}
$$

Applying a solution of the form

$$
\vec{E}(\vec{r}, t)=\vec{\Psi}(\vec{r}) e^{i \omega t}
$$

we have $\nabla^{2} \vec{\Psi}(\vec{r})+\mu_{0} \varepsilon_{0} \omega^{2} \vec{\Psi}(\vec{r})=0$. Defining $\kappa^{2} \equiv \mu_{0} \varepsilon_{0} \omega^{2}$ yields the Helmholtz wave equation

$$
\left(\nabla^{2}+\kappa^{2}\right) \vec{\Psi}(\vec{r})=0
$$

At this point, a plane wave solution is typically introduced. However, the only assumptions we will make is that of a radial dependence in the plane perpendicular to the direction of propagation and cylindrical symmetry. The microwave antenna (Potter horn) and lenses are circular, so the cylindrical symmetry assumption is reasonable. With $z$ as the propagation direction, let

$$
\vec{\Psi}(\vec{r})=U(\rho, z) e^{-i k z} \hat{\Psi}
$$

be the form of the general solution to Equation (6.8). Substituting the Laplacian operator in cylindrical geometry we have

$$
\left[\frac{1}{\rho} \frac{\partial}{\partial \rho}+\frac{\partial^{2}}{\partial \rho^{2}}+\frac{\partial^{2}}{\partial z^{2}}+\kappa^{2}\right] U(\rho, z) e^{-i k z}=0
$$

Applying the operators and doing some algebra we obtain

$$
\frac{1}{\rho} \frac{\partial U(\rho, z)}{\partial \rho}+\frac{\partial^{2} U(\rho, z)}{\partial \rho^{2}}-2 i k \frac{\partial U(\rho, z)}{\partial z}+\frac{\partial^{2} U(\rho, z)}{\partial z^{2}}=0
$$


Under the paraxial approximations: the variation in amplitude $U(\rho, z)$ in the direction of propagation is small compared to a single wavelength $\lambda, 3,4$

$$
\left|\frac{\partial^{2} U(\rho, z)}{\partial z^{2}}\right| \ll\left|2 i k \frac{\partial U(\rho, z)}{\partial z}\right|
$$

and the axial variation in the wave amplitude is small compared to the variation in wave amplitude perpendicular to the direction of propagation,

$$
\left|\frac{\partial^{2} U(\rho, z)}{\partial z^{2}}\right| \ll\left|\frac{1}{\rho} \frac{\partial U(\rho, z)}{\partial \rho}\right|
$$

and

$$
\left|\frac{\partial^{2} U(\rho, z)}{\partial z^{2}}\right| \ll\left|\frac{\partial^{2} U(\rho, z)}{\partial \rho^{2}}\right|
$$

the wave equation becomes:

$$
\frac{1}{\rho} \frac{\partial U(\rho, z)}{\partial \rho}+\frac{\partial^{2} U(\rho, z)}{\partial \rho^{2}}-2 i k \frac{\partial U(\rho, z)}{\partial z}=0 .
$$

Note that the paraxial approximations are equivalent to requiring that $\partial^{2} U(\rho, z) / \partial z^{2}=0$, i.e. the variation in wave amplitude, $U(\rho, z)$, is slow relative to the spatial frequency of the wave, $e^{-i k z} \cdot{ }^{4}$ Assuming a solution of the form

$$
U(\rho, z)=A(z) \exp \left(\frac{-i k \rho^{2}}{2 q(z)}\right)
$$

where $A(z)$ and $q(z)$ are complex functions, and rearranging terms

$$
-2 i k\left[\frac{A(z)}{q(z)}+\frac{\partial A(z)}{\partial z}\right]+\frac{k^{2} \rho^{2} A(z)}{q(z)^{2}}\left[\frac{\partial q(z)}{\partial z}-1\right]=0 .
$$

This complex equation has two terms that must each vanish: 


$$
\frac{\partial q(z)}{\partial z}=1
$$

and

$$
\frac{A(z)}{q(z)}=-\frac{\partial A(z)}{\partial z}
$$

From Equation (6.14), $\partial q(z)=\partial z$. Both sides can be integrated over "dummy limits" $z_{0}^{\prime}$ and $\quad z^{\prime}, \int_{z_{0}^{\prime}}^{z^{\prime}} \partial q(z)=\int_{z_{0}^{\prime}}^{z^{\prime}} \partial z ;$ yielding $q\left(z^{\prime}\right)-q\left(z_{0}^{\prime}\right)=z^{\prime}-z_{0}^{\prime}$. With substitution $z^{\prime} \rightarrow z_{0}$, $z_{0}^{\prime} \rightarrow z$, and $z_{0}=0$,

$$
q(z)=q(0)+z
$$

Equation (6.16) is directly related to the radius of curvature for the propagation of spherical waves. ${ }^{5}$ Since the $1 / q(z)$ term in the exponential argument of Equation 6.12 is complex, we rewrite $1 / q(z)$ as

$$
\frac{1}{q(z)}=\left(\frac{1}{q(z)}\right)_{r}-i\left(\frac{1}{q(z)}\right)_{i}
$$

Substitution of Equation 6.17 into the exponential part of Equation 6.12 yields

$$
\exp \left[\left(\frac{-i k \rho^{2}}{2}\right)\left(\frac{1}{q(z)}\right)_{r}\right]_{\exp }\left[\left(\frac{-k \rho^{2}}{2}\right)\left(\frac{1}{q(z)}\right)_{i}\right] .
$$

The $(1 / q(z))_{r}$ term is related to the beams' radius of curvature, $R(z)$, through

$$
\left(\frac{1}{q(z)}\right)_{r}=\frac{1}{R(z)}
$$

The radius of curvature defines a surface of constant phase relative to a reference plane located at position, $z$, along the direction of propagation. Any point in the beam offset radially, in the $\rho$ direction, from the propagation axis will have a phase lag relative to 
the propagation axis because of the finite radius of curvature of the beam (see Figure $6.1)$.

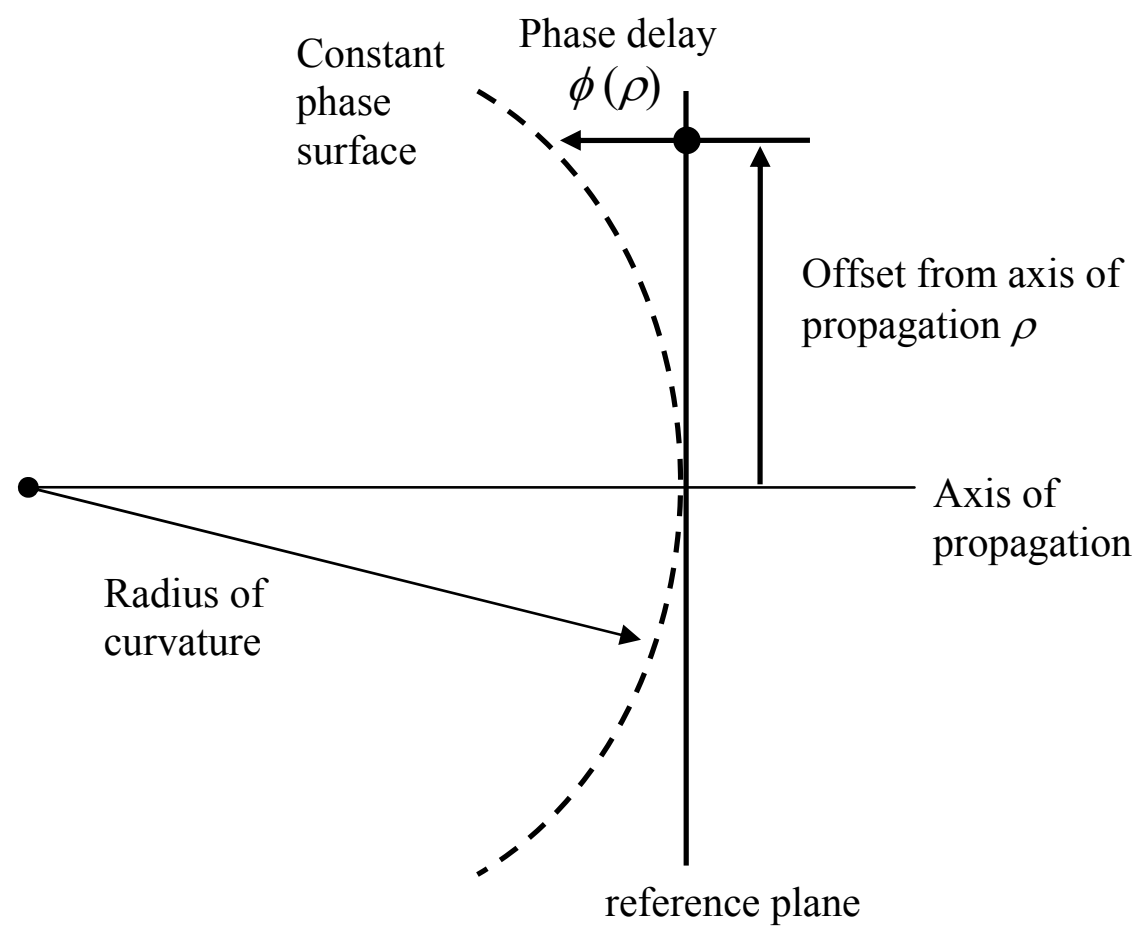

Figure 6.1 Gaussian beam phase shift (delay) relative to that of a plane wave.

The second term in Equation (6.18),

$$
\exp \left[\left(\frac{-k \rho^{2}}{2}\right)\left(\frac{1}{q(z)}\right)_{i}\right]
$$

relates to the variation in wave amplitude as a function of radial distance from the axis of propagation. Note the similarity between Equation (6.20) and the general form of a Gaussian distribution $\exp \left(-\rho^{2} / \rho_{0}^{2}\right)$, where $\rho_{0}$ is defined as the $1 / e$ folding length or waist. Defining $W(z)$ as the beam waist for a given axial position $z$, where the radial field amplitude has decreased by a factor of $1 / e$, 


$$
\left(\frac{1}{q(z)}\right)_{i}=\frac{2}{k W(z)^{2}} .
$$

Substituting $k=2 \pi / \lambda$,

$$
\left(\frac{1}{q(z)}\right)_{i}=\frac{\lambda}{\pi W(z)^{2}}
$$

Using Equations (6.19) and (6.22), Equation (6.17) becomes

$$
\frac{1}{q(z)}=\frac{1}{R(z)}-\frac{i \lambda}{\pi W(z)^{2}},
$$

and Equation (6.12) becomes

$$
U(\rho, z)=A(z) \exp \left[\left(\frac{-i k \rho^{2}}{2}\right)\left(\frac{1}{R(z)}\right)\right] \exp \left[\left(\frac{-k \rho^{2}}{2}\right)\left(\frac{\lambda}{\pi W(z)^{2}}\right)\right] .
$$

Equation (6.24) describes the wave amplitude as a function of $\rho$ and $z$ for a given beams radius of curvature and waist. The boundary condition at $z=0$ for Equation (6.24) is that the beam waist equals the diameter of the launching horn antenna. Although we have yet to determine the explicit form of the radius of curvature $R(z)$, physical intuition suggests that the radius of curvature at the antenna horn would have to be infinity, i.e., $1 / R(z=0)=0$. Defining $W(0) \equiv w_{0}$, where $w_{0}$ is the minimum beam waist at $z=0$, Equation (6.24) becomes

$$
U(\rho, 0)=A(0) \exp \left(\frac{-\rho^{2}}{w_{0}^{2}}\right) .
$$

The same $z=0$ boundary condition applies to Equation (6.12)

$$
U(\rho, 0)=A(0) \exp \left(\frac{-i k \rho^{2}}{2 q(0)}\right) .
$$

Equating the exponential arguments of Equation (6.25) to Equation (6.26) we obtain 


$$
\frac{i \pi \rho^{2}}{\lambda q(0)}=\frac{\rho^{2}}{w_{0}^{2}}
$$

Rearranging, we obtain

$$
q(0)=\frac{i \pi w_{0}^{2}}{\lambda}
$$

Substituting this result into Equation (6.16)

$$
q(z)=\frac{i \pi w_{0}^{2}}{\lambda}+z
$$

thus confirming the assumption that $q(z)$ is complex. Substituting Equation (6.29) into Equation (6.23)

$$
1=i\left[\frac{\pi w_{0}^{2}}{\lambda R(z)}-\frac{\lambda z}{\pi W(z)^{2}}\right]+\left[\frac{w_{0}^{2}}{W(z)^{2}}+\frac{z}{R(z)}\right] .
$$

Satisfying the real and imaginary parts of Equation (6.30), the explicit forms of $W(z)$ and $R(z)$ are determined. From the imaginary term

$$
W(z)^{2}=\frac{\lambda^{2} z R(z)}{\pi^{2} w_{0}^{2}} .
$$

Equation (6.31) and the real terms of Equation (6.30) can be combined into the full expression for the radius of curvature

$$
R(z)=z+\frac{1}{z}\left(\frac{\pi w_{0}^{2}}{\lambda}\right)^{2}
$$

In the limit $z=0$,

$$
\lim _{z \rightarrow 0} R(z)=\lim _{z \rightarrow 0}\left[z+\frac{1}{z}\left(\frac{\pi w_{0}^{2}}{\lambda}\right)^{2}\right] \rightarrow \infty,
$$


the radius of curvature goes to infinity; consistent with the assumption made previously. Substitution of Equation (6.32) into Equation (6.31), yields the explicit form of the Gaussian beam waist

$$
W(z)=w_{0}\left[1+\left(\frac{\lambda z}{\pi w_{0}^{2}}\right)^{2}\right]^{1 / 2} .
$$

Both the radius of curvature and beam waist functions are key to defining and understanding the propagation characteristics of the quasioptical Gaussian beam.

Having nearly exhausted all the information contained in Equation (6.14), it is worthwhile to focus on Equation (6.15). Rearranging terms, we obtain $\partial A(z) / A(z)=-\partial z / q(z) . \quad$ After substitution of Equation $\partial A(z) / A(z)=-\partial q(z) / q(z)$. Integrating both sides,

$$
\int_{z_{0}^{\prime}}^{z^{\prime}} A(z) / A(z)=-\int_{z_{0}^{\prime}}^{z^{\prime}} q(z) / q(z)
$$

to obtain $\ln \left(A\left(z^{\prime}\right) / A\left(z_{0}^{\prime}\right)\right)=\ln \left(q\left(z_{0}^{\prime}\right) / q\left(z^{\prime}\right)\right)$ and replacing the dummy limit values $z^{\prime} \rightarrow z_{0}$ and $z_{0}^{\prime} \rightarrow z$ while setting $z_{0}=0$, we obtain $A(z) / A(0)=q(0) / q(z)$. Substituting Eqs. (6.28) and (6.29)

$$
\frac{A(z)}{A(0)}=\frac{1}{1+\left(\frac{i z \lambda}{\pi w_{0}^{2}}\right)^{2}}\left(1+\frac{i z \lambda}{\pi w_{0}^{2}}\right) .
$$

In this form, our original assumption that $A(z)$ is complex is confirmed.

Additional insight is gained from a phasor analysis of Equation (6.34). Letting

$$
x+i y=|z| \exp (i \phi)
$$




$$
\begin{aligned}
& |z|=\sqrt{x^{2}+y^{2}}, \\
& \phi=\tan ^{-1}\left(\frac{y}{x}\right)
\end{aligned}
$$

and noting that the leading factor in Equation (6.34) equals $\left(w_{0} / W(z)\right)^{2}$ by Equation (6.33), the phasor analysis yields

$$
|z|=\sqrt{1^{2}+\left(\frac{z \lambda}{\pi w_{0}^{2}}\right)^{2}}=\frac{W(z)}{w_{0}}
$$

and

$$
\phi_{0}(z)=\tan ^{-1}\left(\frac{z \lambda}{\pi w_{0}^{2}}\right)
$$

$\phi_{0}(z)$ defines the Gaussian beam phase shift, and is just as important to the beam characterization as the radius of curvature and beam waist parameters. The Gaussian beam phase shift is the on-axis phase relative to that of a plane wave. Substitution of the phasor quantities and assuming $A(0)=1$, Equation (6.34) yields

$$
A(z)=\frac{w_{0}}{W(z)} \exp \left(i \phi_{0}(z)\right)
$$

By determining $A(z)$ we have also determined the explicit form of Equation (6.12), and by substitution of Equation (6.40) we have

$$
U(\rho, z)=\frac{w_{0}}{W(z)} \exp \left(\frac{-i \pi \rho^{2}}{\lambda R(z)}-\frac{\rho^{2}}{W(z)^{2}}+i \phi_{0}(z)\right)
$$

Substitution of this result into Equation (6.8) yields

$$
\vec{\Psi}(\vec{r})=\frac{w_{0}}{W(z)} \exp \left(\frac{-i \pi \rho^{2}}{\lambda R(z)}-\frac{\rho^{2}}{W(z)^{2}}-i k z+i \phi_{0}(z)\right) \hat{\Psi}
$$


The electric field in the beam is then described by

$$
\vec{E}(\vec{r}, t)=\frac{w_{0}}{W(z)} e\left(\frac{-i \pi \rho^{2}}{\lambda R(z)}-\frac{\rho^{2}}{W(z)^{2}}-i k z+i \phi_{0}(z)+i \omega t\right) .
$$

Equation (6.43) is the explicit electric field wave vector for a propagating quasioptical Gaussian beam in the paraxial limit.

In terms of diagnostic design, the three fundamental beam characteristic parameters and the electric field distribution are all important quantities to consider in both the near and far field limits. The quantity,

$$
z_{c}=\frac{\pi w_{0}^{2}}{\lambda},
$$

typically referred to as the confocal parameter or the "Rayleigh length" in the literature; separates the "near field" $\left(z<<z_{c}\right)$ and "far field" $\left(z>>z_{c}\right)$ characteristic zones for the $R(z), W(z)$, and $\phi_{0}(z)$ beam parameters. Substitution of the confocal parameter into the previously derived expressions yields

$$
\begin{aligned}
& R(z)=z+\frac{z_{c}^{2}}{z}, \\
& W(z)=w_{0}\left[1+\left(\frac{z}{z_{c}}\right)^{2}\right]^{1 / 2},
\end{aligned}
$$

and

$$
\phi_{0}(z)=\tan ^{-1}\left(\frac{z}{z_{c}}\right) .
$$

In the near field and far field limits, the radius of curvature, becomes

$$
\lim _{z<<z_{c}} R(z)=\lim _{z<<z_{c}}\left[z+\frac{z_{c}^{2}}{z}\right] \rightarrow \frac{z_{c}^{2}}{z}
$$


and

$$
\lim _{z>z_{c}} R(z)=\lim _{z>>z_{c}}\left[z+\frac{z_{c}^{2}}{z}\right] \rightarrow z
$$

respectively. In the near field the curvature goes as $1 / z$, and as required, the radius of curvature remains infinite at $z=0$. The transition from near field to far field occurs at $Z \sim Z_{c}$, where the curvature radius equals twice the distance the beam has propagated. In the far field, the curvature becomes linear with propagation distance.

In the near and far field, the beam waist limits become

$$
\lim _{z<<z_{c}} W(z)=\lim _{z<z_{c}}\left[w_{0}\left[1+\left(\frac{z}{z_{c}}\right)^{2}\right]^{1 / 2}\right] \rightarrow w_{0},
$$

and

$$
\lim _{z>z_{c}} W(z)=\lim _{z>>z_{c}}\left[w_{0}\left[1+\left(\frac{z}{z_{c}}\right)^{2}\right]^{1 / 2}\right] \rightarrow w_{0} \frac{z}{z_{c}},
$$

respectively. In the near field, the beam waist remains essentially the same size as the launching antenna. At the transition from the near to far field $z \sim Z_{c}$, the waist has only increased by a factor of $\sqrt{2}$ over the original beam waist $w_{0}$. In the far field, the beam waist increases linearly with propagation distance.

For any Gaussian beam propagating through free space that has been focused to a minimum beam waist, the region defined by $w_{0} \leq w(z) \leq \sqrt{2} w_{0}$ (see Figure 6.2) is called the "Rayleigh length" $\left(R_{L}\right)$. The Rayleigh length is the distance on either side of the minimum waist where the beam waist is essentially constant. Note that Gaussian beams do not achieve a pinpoint focus, as in geometrical optics. Instead there are regions of 
minimal beam waist size. The dashed lines crossing at where the beam waist is a minimum define the asymptotic beam growth angle $\Theta$ in Figure 6.2. The asymptotic beam growth angle, also known as the far field divergence angle, is defined as

$$
\tan \Theta=\frac{W(z)}{Z},
$$

and is the angle between the propagation axis and the far field asymptotic limit of the beam waist. Recalling the far field limit of the beam waist and the definition of the confocal parameter, Equation (6.48) becomes

$$
\Theta=\tan ^{-1}\left(\frac{\lambda}{\pi w_{0}}\right)
$$

In terms of real quantities, for $\lambda=1 \mathrm{~mm}$ and $w_{0}=1.9 \mathrm{~mm}$ (specifications of the WVU $300 \mathrm{GHz}$ source), $\lambda / \pi w_{0} \approx 0.17$. Employing the small angle approximation, $\Theta \cong \lambda / \pi w_{0} \cong 0.17$ radians

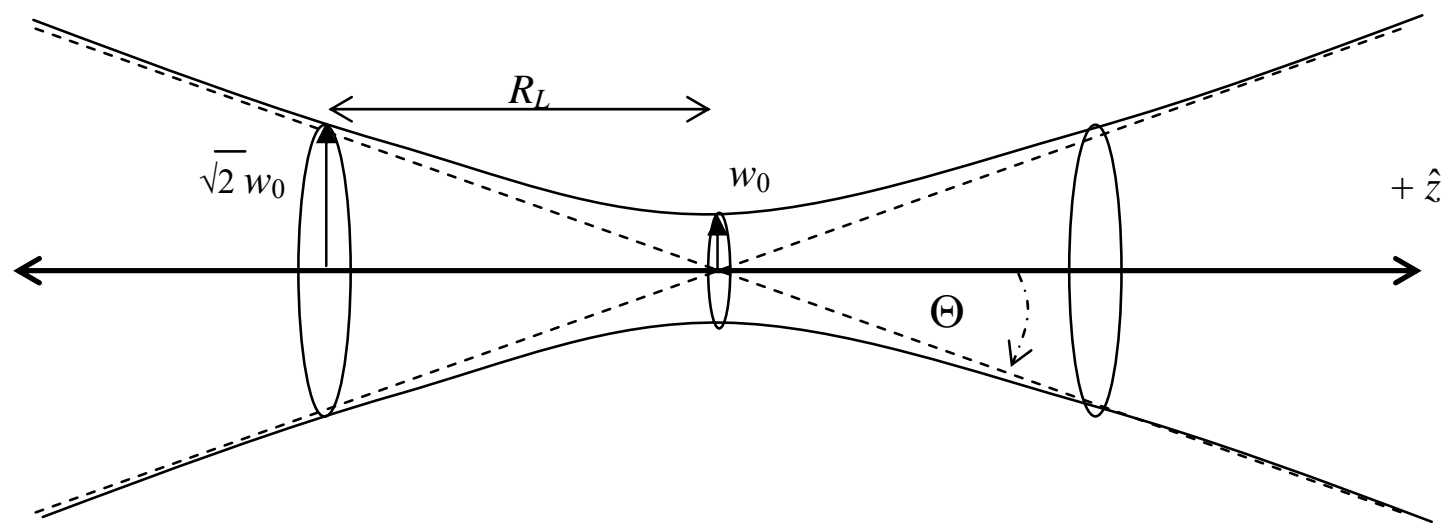

Figure 6.2 Gaussian beam converging and diverging from a minimum beam waist.

The limits of the beam phase shift, in both the near and far field are

$$
\lim _{z<<z_{c}} \phi_{0}(z)=\lim _{z<<z_{c}}\left[\tan ^{-1}\left(\frac{z}{z_{c}}\right)\right] \rightarrow 0
$$

and 


$$
\lim _{z>z_{c}} \phi_{0}(z)=\lim _{z>z_{c}}\left[\tan ^{-1}\left(\frac{z}{z_{c}}\right)\right] \rightarrow \frac{\pi}{2},
$$

respectively. As expected, the phase shift difference from a plane wave is zero in the near field. At the transition point between the near and far field $z \sim Z_{c}$, the phase shift becomes $\pi / 4$. In the far field, the difference in phase from the standard plane wave solution becomes $\pi / 2$.

The final Gaussian beam quantity of importance is the spatial distribution of the beam power. As in a plane wave, the power in a Gaussian beam is proportional to the square of the electric field amplitude. Setting the total squared electric field, integrated over radial position, to unity

$$
\int_{0}^{\infty}|E|^{2} 2 \pi \rho \partial \rho=1
$$

the electric field expression of Equation (6.43) becomes

$$
\vec{E}(\vec{r}, t)=\sqrt{\frac{2}{\pi W(z)^{2}}} \exp \left(\frac{-i \pi \rho^{2}}{\lambda R(z)}-\frac{\rho^{2}}{W(z)^{2}}-i k z+i \phi_{0}(z)+i \omega t\right) .
$$

Relative to the electric field amplitude along the axis of propagation,

$$
\frac{|E(\rho, z)|}{|E(0, z)|}=\exp \left(\frac{-\rho^{2}}{W(z)^{2}}\right)
$$

Since power is proportional to the square of the electric field distribution,

$$
\frac{P(\rho, z)}{P(0, z)}=\frac{|E(\rho, z)|^{2}}{|E(0, z)|^{2}}=\exp \left(\frac{-2 \rho^{2}}{W(z)^{2}}\right)
$$

From Equation (6.50), $|E(0, z)|=\sqrt{2 / \pi W(z)^{2}}$, thus 


$$
P(\rho, z)=\left(\frac{2}{\pi W(z)^{2}}\right) \exp \left(\frac{-2 \rho^{2}}{W(z)^{2}}\right)
$$

in a Gaussian beam at some radial position, $\rho$, and some axial location, $z$, along the beam.

\subsection{GHz Diagnostic Design}

Three general requirements drove the initial design and construction of the 300 GHz CTS diagnostic. First and foremost was system portability. All optical components, except a collection mirror, had to fit on a 36 " by 36 " vertically mounted optical table. The second design constraint was the need to aim the interaction beam into the plasma at different angles for measurement at a variety of scattering angles and plasma locations. The third design constraint was to produce an interaction beam with a minimum Rayleigh length of at least the plasma column diameter.

Scattering system design including the design of the lenses was accomplished with two computer programs: ZEMAX by ZEMAX Development Corporation, and Graphite by Ashlar-Vellum, Inc. ZEMAX is an optical design code capable of quasioptical Gaussian beam propagation and Graphite is a three-dimensional, wire-frame, Computer-Aided Design (CAD) program. Before describing the details of the system development in ZEMAX, we first need to review details of the source and detector selected for these experiments.

\subsection{1 $300 \mathrm{GHz}$ Source and Detector}

The scattering diagnostic is designed around a $300 \mathrm{GHz} \mathrm{mm}$-wave source and detector. The source is manufactured by Radiometer Physics GmbH in Meckenheim, 
Germany. The principle emitter is a Gunn oscillator with a resonant cavity that can be tuned over a small frequency range, 99.5 to $100.5 \mathrm{GHz}$. It is coupled into a "frequency tripler," a second cavity that mode converts the original wave to a 299 to $301.5 \mathrm{GHz}$ wave. The high frequency wave then propagates down a small WR-3 fundamental rectangular waveguide to a Potter horn ${ }^{6}$ antenna. Frequency tuning is accomplished by applying a bias voltage between 0 and 8 Volts through the Varactor diode input. The 300 $\mathrm{GHz}$ source tuning characteristics are given in Table 6.1. The source uses the Potter horn antenna to produce the cylindrically symmetric Gaussian beam mode. The beam is linearly polarized with the electric-field-vector perpendicular to the base of the source and has a quoted waist $\left(w_{0}\right)$ of $0.19 \mathrm{~cm}$. With a known minimum beam waist, the ZEMAX ray tracing software can be used to model the optical components of the full system. Recently, a nearly identical source has been used as an interferometer for time and space resolved electron density measurements on a pulsed helicon source. ${ }^{7}$

Table 6.1 $300 \mathrm{GHz}$ Source Characteristics ${ }^{8}$

\begin{tabular}{|c|c|c|c|}
\hline \multicolumn{2}{|c|}{ Source Output } & \multicolumn{2}{c|}{ Gunn Oscillator } \\
\hline Frequency [GHz] & Output Power [mW] & Supply Voltage [V] & Varactor Voltage [V] \\
\hline 299.1 & 4.20 & 10.60 & 7.90 \\
\hline 299.4 & 3.80 & 10.60 & 7.47 \\
\hline 299.7 & 3.50 & 10.60 & 7.33 \\
\hline 300.0 & 3.30 & 10.60 & 6.45 \\
\hline 300.3 & 3.25 & 10.60 & 5.90 \\
\hline 300.6 & 3.15 & 10.60 & 5.35 \\
\hline 300.9 & 3.00 & 10.60 & 4.70 \\
\hline
\end{tabular}

The detector, also from Radiometer Physics GmbH, is a Crystal Schottky diode detector-mixer centered at $300 \mathrm{GHz}$ with an intermediate frequency (IF) bandwidth of approximately $500 \mathrm{MHz}$ and a quoted sensitivity of $600 \mathrm{mV} / \mathrm{mW}$. The detector, like the source, has a Potter horn to collect the radiation. Assuming that the beam waist at the 
detector horn must be the same size as the output waist of the source, defines how the optics must be designed to focus the scattered radiation into the detector. The detectormixer bias characteristics are provided in Table 6.2, and pictures of the source and detector are shown in Figure 6.3.

Table 6.2 $300 \mathrm{GHz}$ Detector Diode Characteristics ${ }^{8}$

\begin{tabular}{|c|c|c|c|c|}
\hline Bias Voltage $[\mathrm{V}]$ & -0.61 & -0.69 & -0.79 & -0.89 \\
\hline Current $[\mu \mathrm{A}]$ & -10 & -100 & -1000 & -3000 \\
\hline
\end{tabular}
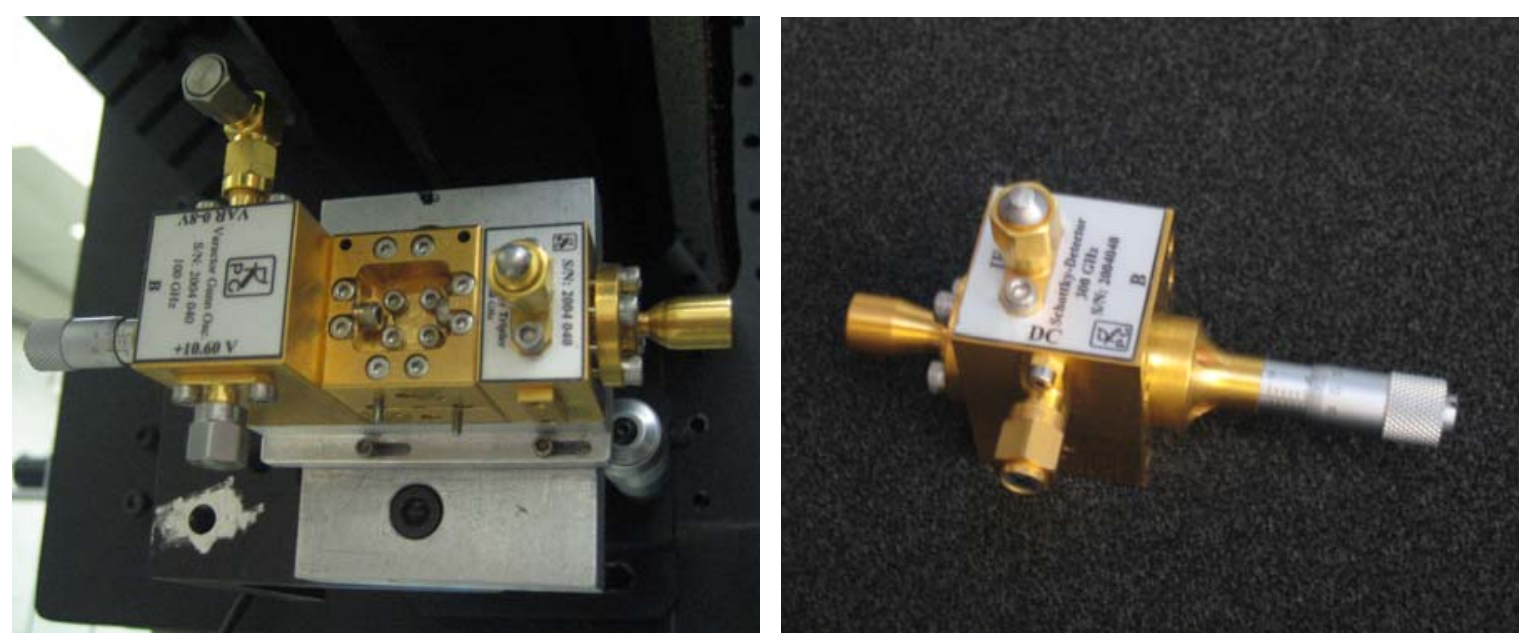

Figure 6.3 The $300 \mathrm{GHz}$ source and detector.

\subsubsection{Optical Design}

ZEMAX is an optical modeling computer program that calculates electromagnetic propagation of a Gausian beam through a series of surfaces. The program permits a large number of shaped and tilted surfaces to be modeled, while simultaneously providing a complete analysis of the optical system. It produces CAD-like schematics of the optical elements, and includes the capability to export the files in the DXF format that can be read into Graphite. ZEMAX also calculates a number of parameters that are recorded in a 
formatted spreadsheet for each optical component: the radius of curvature, beam waist, Rayleigh length, and phase shift.

One disadvantage of the ZEMAX version that was purchased is that it will only calculate sequential optics, i.e., a serial progression from one surface through to the next surface, without calculating if there are intervening obstructions or surfaces that may terminate or split the ray path, such as a beam splitter. This limitation necessitated dividing the diagnostic into three distinct branches, the "interaction" beam path, a "scattered" beam path, and a "reference" beam path. A wireframe rendering of the interaction beam path is shown in Figure 6.4. The full model could have been split into only two sections, but the difference in waist sizes at the scattering volume in the plasma made subdividing the design into three sections a simpler approach. By exporting the CAD-like designs into Graphite, the individual beam paths can be combined into a single continuous model, allowing for a complete overview of the entire diagnostic (see Figure 6.5). Because the lenses must have non-spherical surfaces to correct for phase lag due to the Gaussian nature of the beam, exporting the lens drawings to Graphite also helps in the creation of accurate schematics for manufacturing. Note that many of the features in ZEMAX can only be used with the Extended Edition, such as the 'SOLVES' and optimization routines that are not set up for Gaussian beam optimization in our version of the code. Because of the limited features, the development of the individual optical paths required a trial and error approach. Since this is a quasioptical system, all the optical components dimensions were designed so that their radii were at least a factor of two larger than the beam waist at that particular location in the scattering system. 


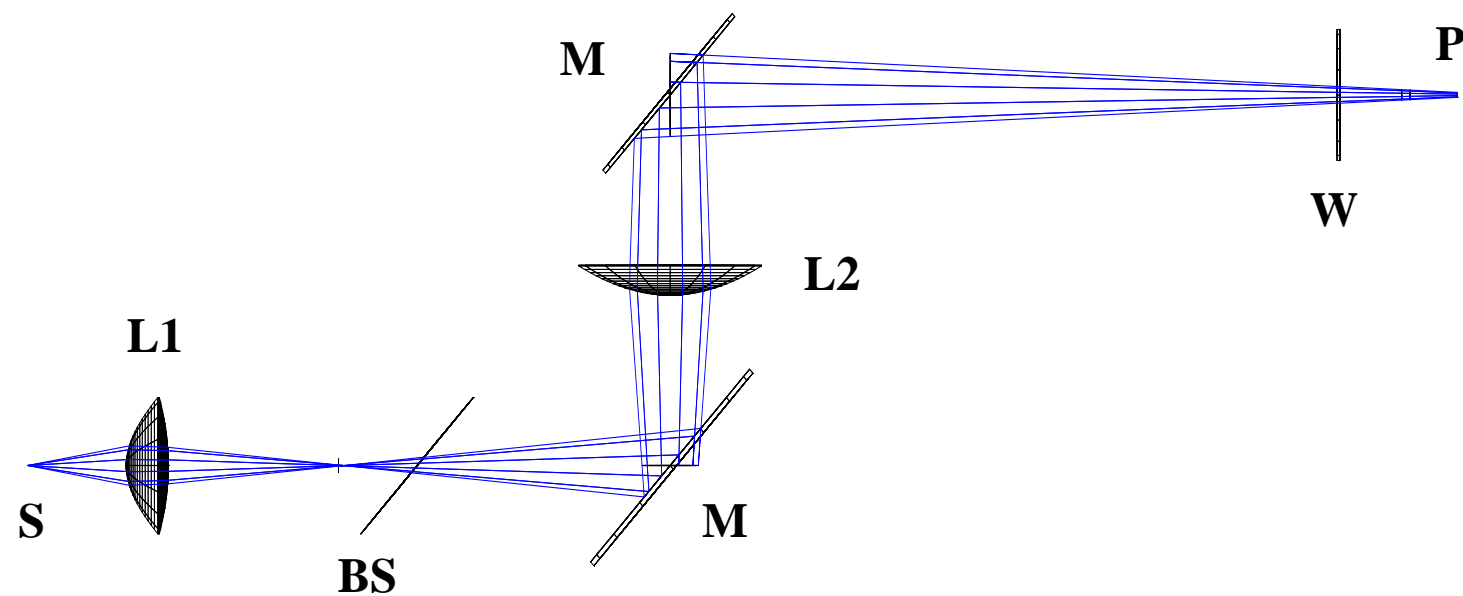

Figure 6.4 ZEMAX wireframe rendering of the interaction beam. S is the source, L1 and L2 are the HDPE lenses, $\mathrm{BS}$ is the beam splitter, $\mathrm{M}$ are the mirrors, $\mathrm{W}$ is the vacuum window, and $\mathrm{P}$ is the plasma.

The interaction beam path begins with the millimeter wave source, followed by a set of primary focusing lenses, a beam splitter, folding mirror, secondary focusing lenses, and a final steering mirror. Due to the quasioptical properties of the $300 \mathrm{GHz}$ source, high density polyethylene (HDPE) lenses are employed for focusing and collimation of the beam. At frequencies of $300 \mathrm{GHz}$, HDPE has an index of refraction of approximately 1.525 as calculated (and used) in ZEMAX based on Palik. ${ }^{9}$ Since the beam is Gaussian, the lenses must have non-spherical surfaces to line up the phase fronts of the beam. Hyperbolic and ellipsoidal lens surfaces are used to produce and maintain a planar phase front and correct any phase lag. An example of the wave front phase analysis routine in ZEMAX, which calculates the beams relative phase front across the beam waist at an optical surface, is shown in Figure 6.6. The phase front produced by the lenses in the interaction beam path is planar at the center of HELIX. HDPE windows are also used as vacuum interfaces for the interaction and scattered beams since standard viewports heavily attenuated the $300 \mathrm{GHz}$ signal. The windows have a $2.5^{\circ}$ wedge cut into each face, relative to the surface normal, to minimize direct reflections. The lenses were 
manufactured directly from $\mathrm{CAD}$ drawings on a $\mathrm{CNC}$ lathe at the Robert $\mathrm{C}$. Byrd institute in Bridgeport, WV.

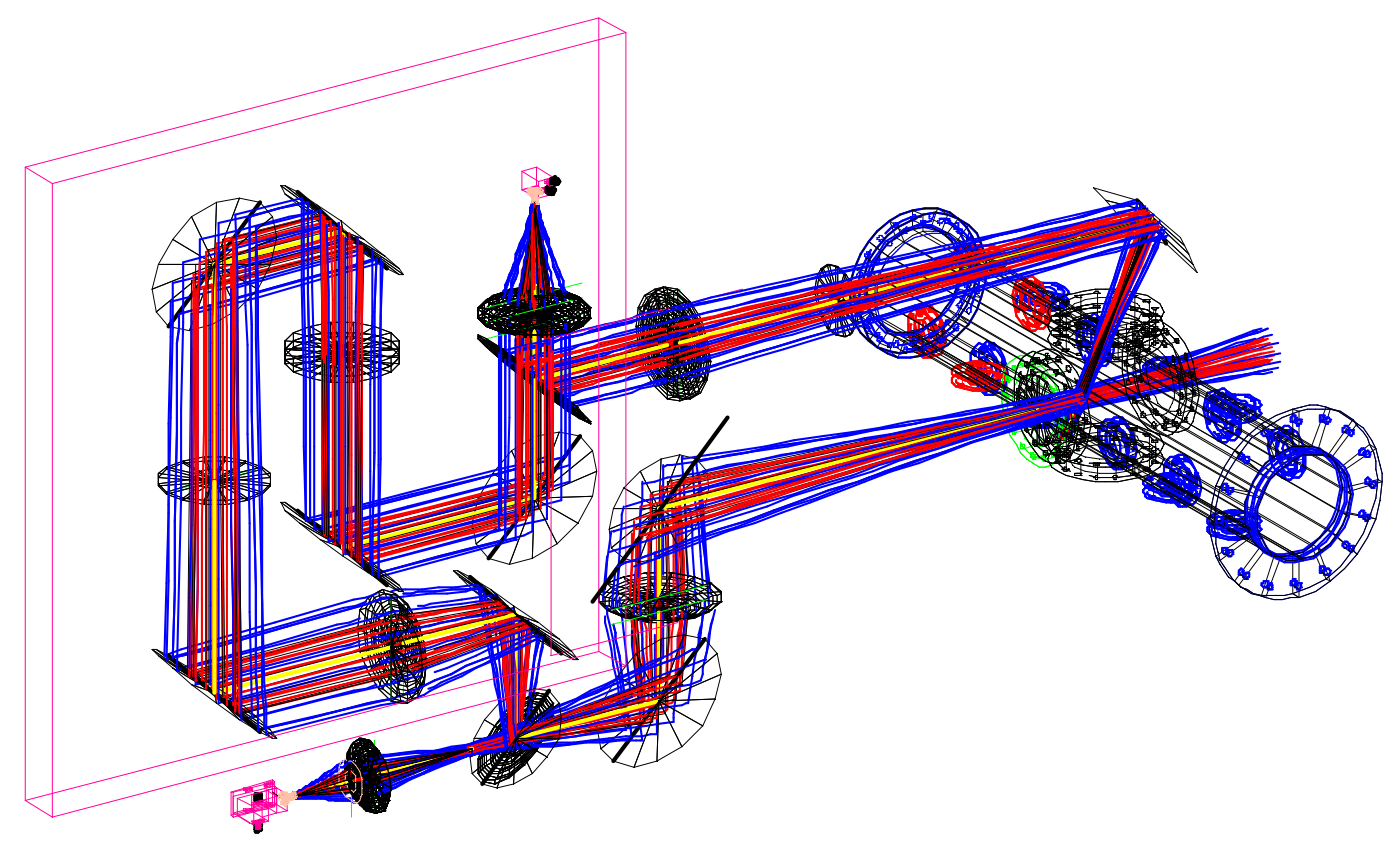

Figure 6.5 The individual beam paths from ZEMAX including HELIX for reference. The colored wire frames indicate the center of the beam (yellow line), the $1 / e$ beam waist (red lines), and the $1 / e^{2}$ beam waist.

Beam splitting is accomplished with stretched Mylar ${ }^{\mathrm{TM}}$ sheets. At a frequency of $300 \mathrm{GHz}$, Mylar has an index of refraction of approximately 1.773. In Figure 6.7, BS1 (Beam splitter \#1) is a $76.2 \mu \mathrm{m}$ thick Mylar sheet that yields a 90-10 (TransmissionReflection) ratio neglecting losses. The 10 percent reflection creates the local oscillator (LO) reference beam while the remaining power is directed into the plasma as the interaction beam. The scattered and reference beams are recombined at BS2 with a 127 $\mu \mathrm{m}$ thick Mylar sheet. The $127 \mu \mathrm{m}$ thickness was found to produce the optimal scattered beam reflection, while minimizing transmission losses. The secondary focusing lenses are designed for focusing the interaction beam, which is optimized so that the Rayleigh length encompasses the entire plasma column. The final component in the primary path is 
the steering mirror. The steering mirror's role is to guide the beam into the plasma. The $300 \mathrm{GHz}$ beam is directed into the plasma with the polarization direction parallel to the magnetic field of HELIX (O-mode propagation) and with a beam waist predicted by ZEMAX to be $17 \mathrm{~mm}$.

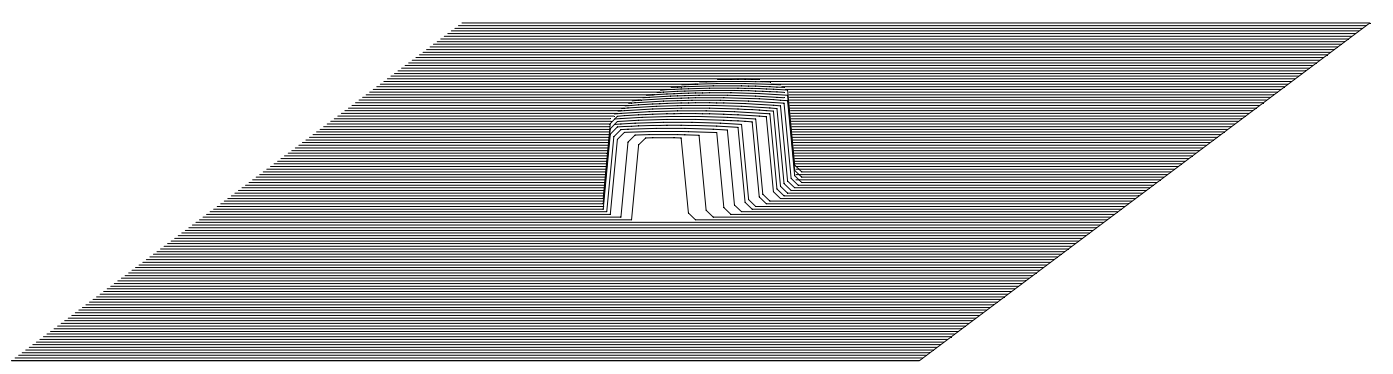

Figure 6.6 The wave front phase analysis of the interaction beam at the center of HELIX.

The scattered beam path, from the plasma to the detector, consists of a vacuum collection mirror, a set of collection lenses, a beam splitter-mixer, a final set of focusing lenses, and the detector. The scattered radiation from the fluctuations in the plasma is directed out of the plasma chamber to the detector by the vacuum collection mirror. For the initial experiments with the system, a flat mirror is used to collect the scattered radiation. Future measurements with improved spatial resolution will employ an off axis parabolic mirror, with the focal point fixed in the interaction beam. The collection lenses are designed to match the scattered beam and the reference beam sizes at the mixer. The beam, once mixed at BS2, is focused into the detector's potter horn with a final set of focusing HDPE lenses. 


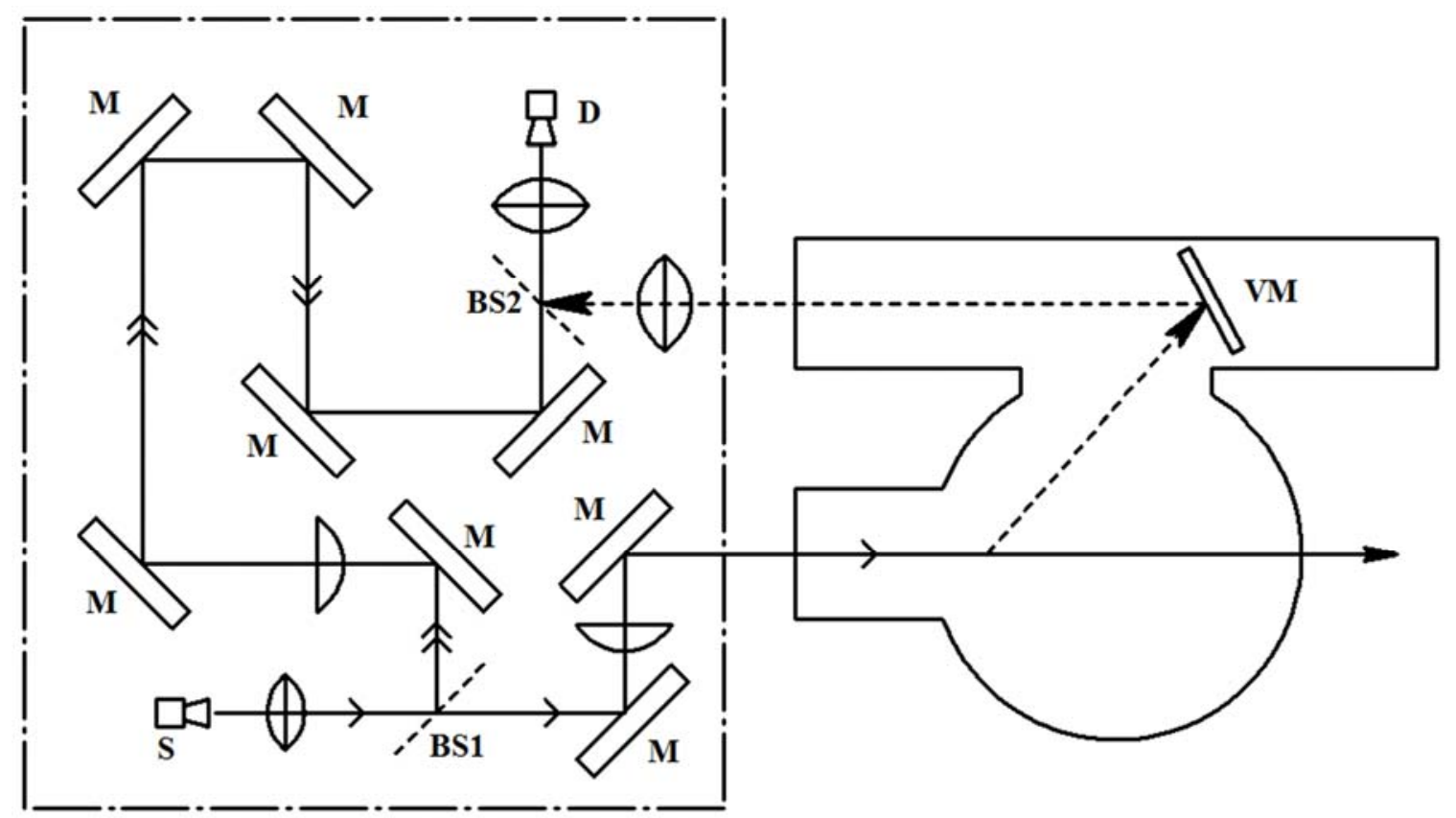

Figure 6.7 Complete schematic of the $\mathrm{mm}$-wave system: (S) is the mm-wave source, (D) is the detector, (M) are mirrors, (VM) is the adjustable vacuum mirror, (BS1) and (BS2) are the beam splitters.

The reference path, after being split from the interaction beam, consists of several folding mirrors, and collimation optics. The purpose of the folding mirrors is to maintain equality of the optical path lengths, to within a single wavelength $(1 \mathrm{~mm})$. Two of the folding mirrors are mounted on a single linear stage for the reference beam path length relative to the primary and scattered path length. The mirrors on the optical table are constructed from mirrored stainless steel sheets attached to aluminum plates with retaining rings and range from 8 to 12 inches in diameter. A picture of the completed diagnostic system as installed is shown in Figure 6.8. 


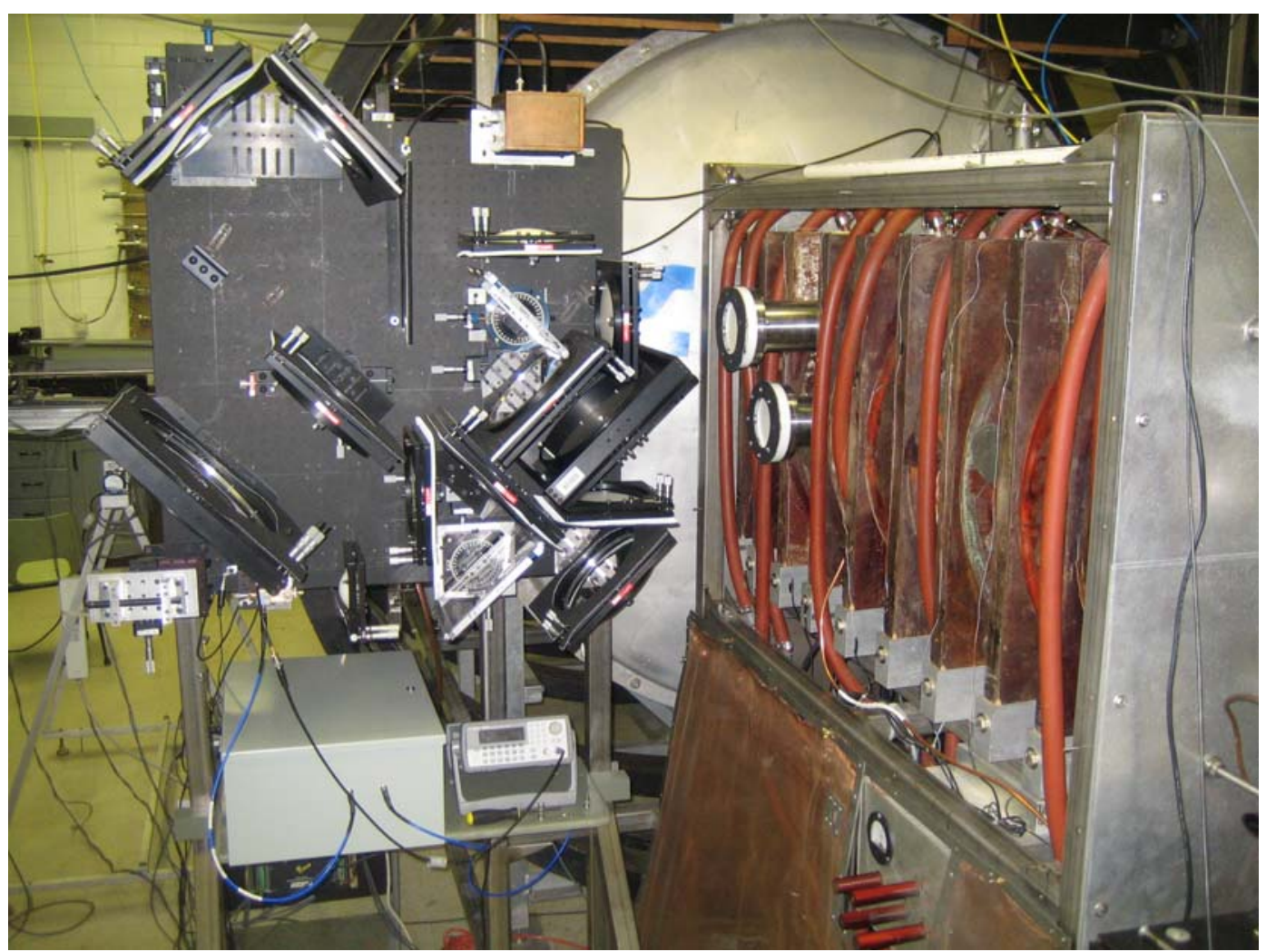

Figure 6.8 The $300 \mathrm{GHz}$ system completed and installed on HELIX.

\subsection{Installation and Testing of the Scattering System and Components}

As each component of the scattering diagnostic was manufactured or purchased, it was tested to make sure it met the predicted characteristics as defined by ZEMAX. The lenses, vacuum windows, and beam splitters were tested using the apparatus shown in Figure 6.9. Because the detector is a mixer, testing was accomplished by chirping the Varactor input voltage on the $300 \mathrm{GHz}$ source with a function generator and using a HP3585A spectrum analyzer to measure the peak output voltage of the detector at the chirping frequency. The source location was fixed while the detector was mounted on a linear translation stage to sweep perpendicularly through the beam. 


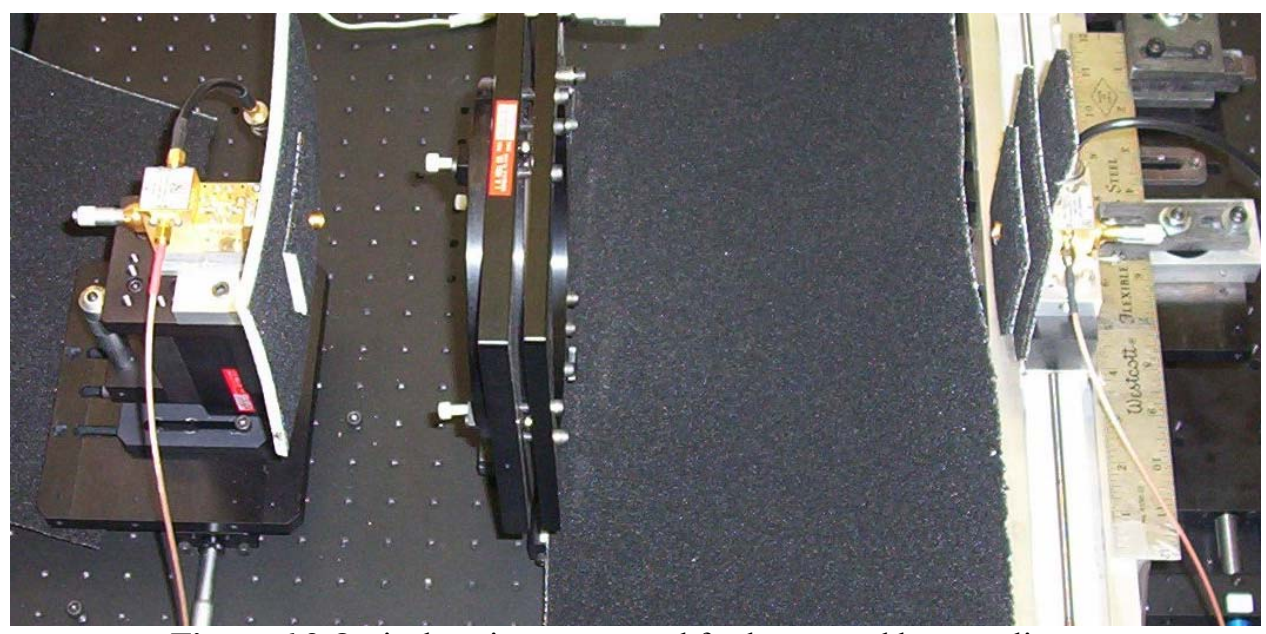

Figure 6.9 Optical testing setup used for lenses and beam splitters.

\subsubsection{HDPE Lenses and Windows}

The optical properties of each custom manufactured lens were compared to the predictions obtained with ZEMAX. Figure 6.10 shows test results for four of the lenses. The data shown in Figure 6.10(a) demonstrate excellent agreement between the measured and predicted beam width, while the lens data shown in Figure 6.10(b) indicate a measured beam width slightly narrower than the prediction. The narrow width most likely arises from an error in the measurement of the distance between the source and lens or the lens and detector and is not a significant cause for concern. The measurement of the beam widths in Figure 6.10(c) and 6.10(d) were cause for concern because of the significant decrease in the central beam power. Several tests were conducted to determine the source of the problem including the effect of the lens mounts, alignment and tilting of the lens, as well as the choice of the incident surface of the plano-convex lens. No significant difference in the central beam power was detected from any of these tests. However, later experiments determined that the problem resulted from a destructive interference effect caused by the quasioptical beam propagation characteristics. Because each lens was tested independently (source - lens - detector), all the lenses except the 
first lens 6.10(a), did not have the appropriate surface curvature to match the curvature of the beam. Even if a lens were placed with the focal point at the beam source, the beam curvature will not necessarily match the curvature of the lens. Thus, multiple reflections could arise. After this discovery, all testing of the lenses was accomplished by incrementally inserting the lenses in the appropriate order. No other significant interference effects were seen in the later lens tests.
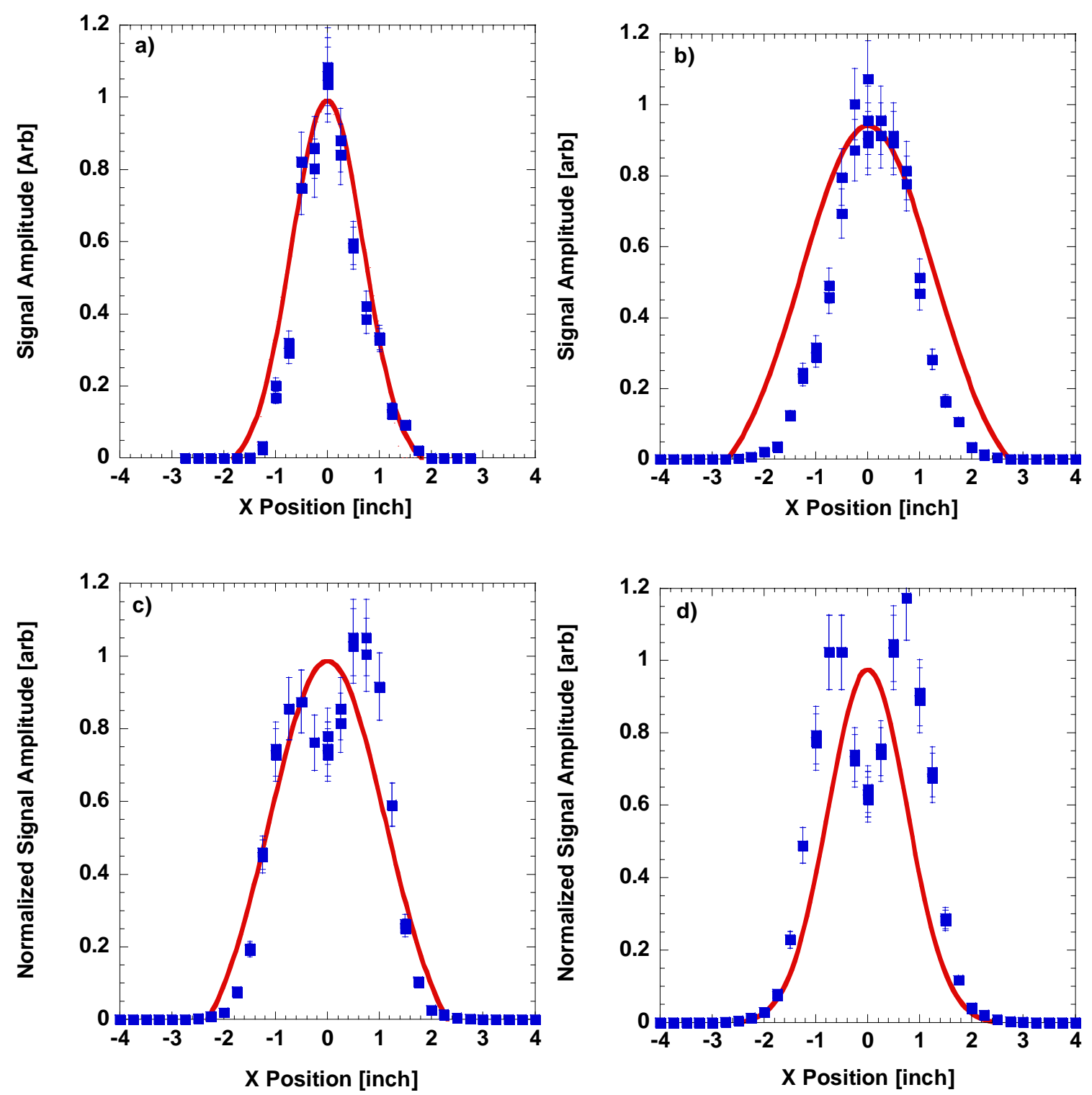

Figure 6.10 Comparison of the predicted ZEMAX (__) and experimentally measured ( $\square$ ) beams. (a) First lens from the source, (b) second lens from the source, (c) last lens in the interaction beam line, and (d) first lens in the reference beam line. 
Due to the need for coupling of the injection and scattered beam from air to vacuum, testing of the transmission through standard vacuum windows initially caused concern due to the less than $5 \%$ transmission observed for standard fused silica windows (Figure 6.11). A $1 / 2$ inch thick slab of HDPE was then tested and found to provide greater than $100 \%$ transmission due to a slight focusing effect. The HDPE windows include a $2.5^{\circ}$ wedge cut into each face, relative to the surface normal, to minimize direct reflections.

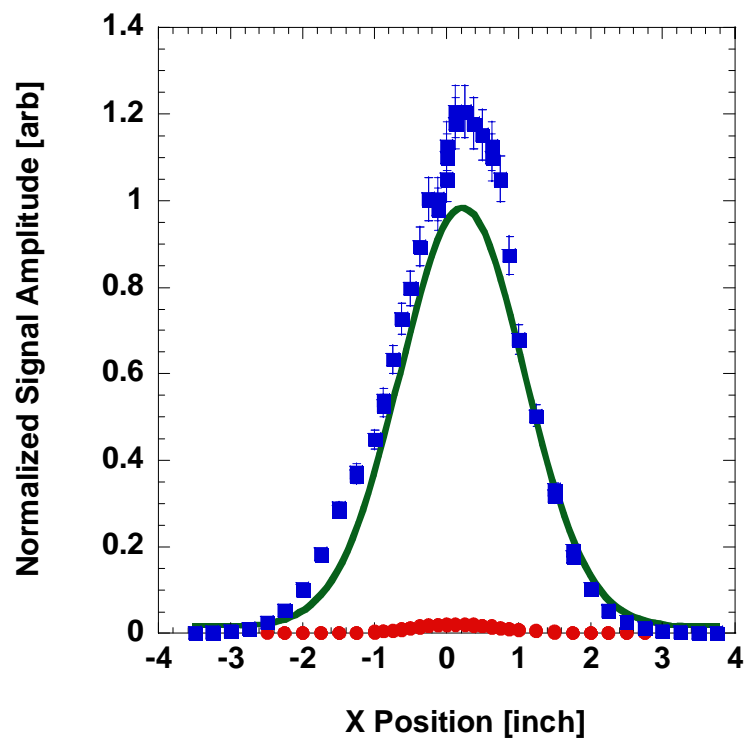

Figure 6.11 Comparison of the transmission of the $300 \mathrm{GHz}$ through air (_), a standard vacuum window port (๑), and a $1 / 2$ inch thick HDPE slab (ロ).

\subsubsection{Beam Splitters}

ZEMAX predicted the Mylar thickness needed to be on the order of $38.1 \mu \mathrm{m}$ to produce a 90-10 (Transmission-Reflection) ratio for the first beam splitter. Since the Mylar sheets were only available in certain thicknesses, several thicknesses were tested to determine the best transmission to reflection ratio, while minimizing losses (see Figure 6.12). 

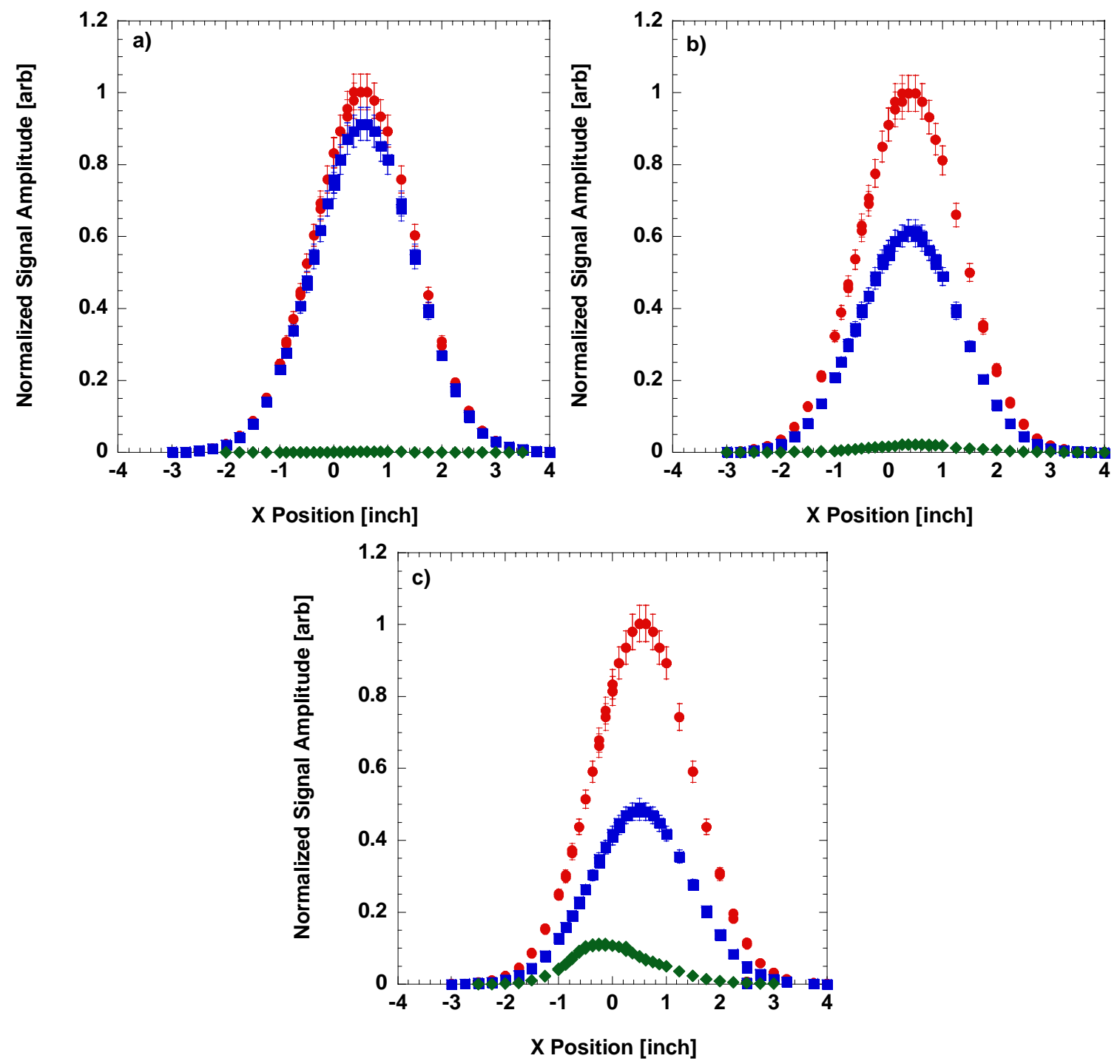

Figure 6.12 Mylar beam splitter thickness comparison at $45^{\circ}$ incidence: (a) $25.4 \mu \mathrm{m}$, (b) $50.8 \mu \mathrm{m}$, and (c) $76.2 \mu \mathrm{m}$. ( $\bullet$ ) is the transmission through air (for normalization), ( $\square$ ) is the transmission coefficient, and $(\diamond)$ is the reflection coefficient.

The thinnest Mylar sheet, with a thickness of $25.4 \mu \mathrm{m}$, had a transmission coefficient of 90\%, but the reflection coefficient was essentially zero (Figure 6.12(a)). The difference in the sum of the transmission and reflection coefficients from $100 \%$ is considered to be from losses in the material or spurious reflections. For a Mylar thickness of $50.8 \mu \mathrm{m}$ Figure $6.12(\mathrm{~b})$, there was an increase in the reflection coefficient to $\sim 3 \%$, but the transmission coefficient decreased to $\sim 60 \%$. The reflection coefficient increased 
further when the Mylar thickness was increased to $76.2 \mu \mathrm{m}$, as shown in Figure 6.12(c), but the transmission coefficient decreased to about $50 \%$. The relative decrease in the transmission coefficient from increasing the sheet thickness from 50.8 to $76.2 \mu \mathrm{m}$ was not as severe as the change going from 25.4 to $50.8 \mu \mathrm{m}$. The larger reflection coefficient of $\sim 10 \%$ for the $76.2 \mu \mathrm{m}$ thick Mylar sheet allowed sufficient power to be maintained in the reference beam; which must pass through the beam combining Mylar sheet to be mixed in the mixer-detector. A Mylar sheet with a thickness of $127 \mu \mathrm{m}$ was chosen for BS2 due to the increase in the reflection coefficient and the acceptable losses in the transmission coefficient. The proof-of-concept test for the entire system, described later in this chapter, confirmed that the beam mixer transmits and reflects enough beam power to produce a detector output signal with a simulated scattered signal.

\subsubsection{Vacuum Collection Mirror Apparatus}

The final optical component to be reviewed is the mirror that directs the scattered radiation from the vacuum chamber back to the optical table. In the vacuum chamber, a flat mirror, designed to maximize signal detection, is used to direct the scattered radiation from the vacuum chamber to the optical table (Figure 6.13). The mirror is oval in shape, to allow linear translation and rotation in the 4 in. diameter vacuum tube while maximizing the reflective surface area. The translation, via two shafts mounted on a linear stage, allows for scanning the collection volume across the plasma column. Radial scanning across the plasma is critical since we expect the slow wave to be largest near the plasma edge. The mirror pivots by sliding along a fitting attached to the end of one shaft while the relative position of other shaft controls the angle of the vacuum mirror through a 
micrometer and an inline ball joint. The micrometer provides an accurate measure of the relative position of the two shafts controlling the angle of the mirror. The inline ball joint converts the rotation and linear translation of the micrometer to only a linear translation for the shaft. Rotating the mirror changes the angle of collection and thereby selects the wave number to be observed. The measurable scattered angles in HELIX range from $60^{\circ}$ to $90^{\circ}$; corresponding to fluctuation wave numbers of 63 to $89 \mathrm{rad} / \mathrm{cm}$ via the Bragg condition, $k=2 k_{0} \sin \left(\theta_{s} / 2\right)$, where $k_{0}$ is the incident wave number, $k$ is the fluctuation wave number, and $\theta_{s}$ is the scattering angle. ${ }^{14}$ The radial location of the collection volume depends on the angle and location of the collection mirror. For the range of observable scattered angles, the collection volume does not change significantly.

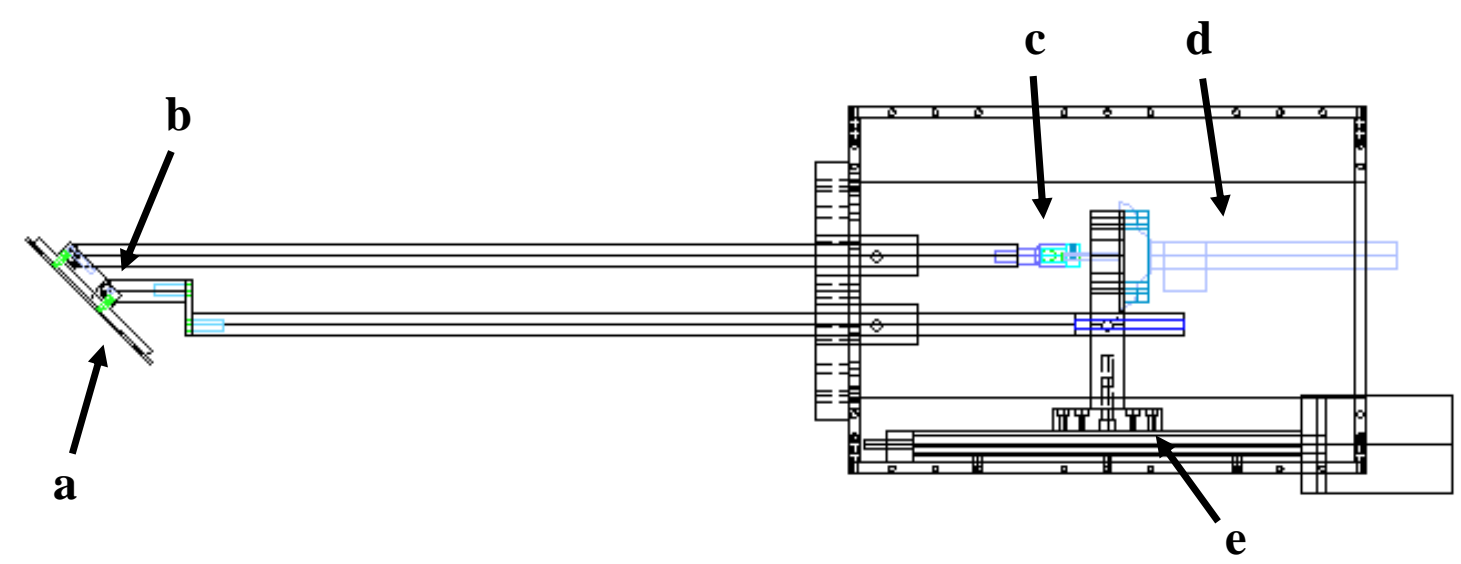

Figure 6.13 Vacuum mirror apparatus. (a) Mirrored aluminum mirror, (b) mirror pivot and slider, (c) inline ball joint (turns rotational drive to linear translation), (d) micrometer, and (e) Velmex stage and shaft mounting.

Mirrored aluminum alignment wedges cut to simulate scattering angles of 60,70 , 80 , and 90 degrees were installed at the target location to evaluate the spatial resolution, as well as to determine a rough estimate of the observable collection beam waist of the collection optics. Observable radii for $60^{\circ}$ scattering are from $r=-5$ to $-1.5 \mathrm{~cm}$, while for $90^{\circ}$ scattering the observable radii range from -1.5 to $+5 \mathrm{~cm}$ (see Figure 6.14). For 
scattering between $60^{\circ}$ and $70^{\circ}$, the collection coverage is limited to only about half the plasma radius, while collection of scattered microwave signal from $80^{\circ}$ to $90^{\circ}$ can be accomplished for nearly the entire plasma diameter.

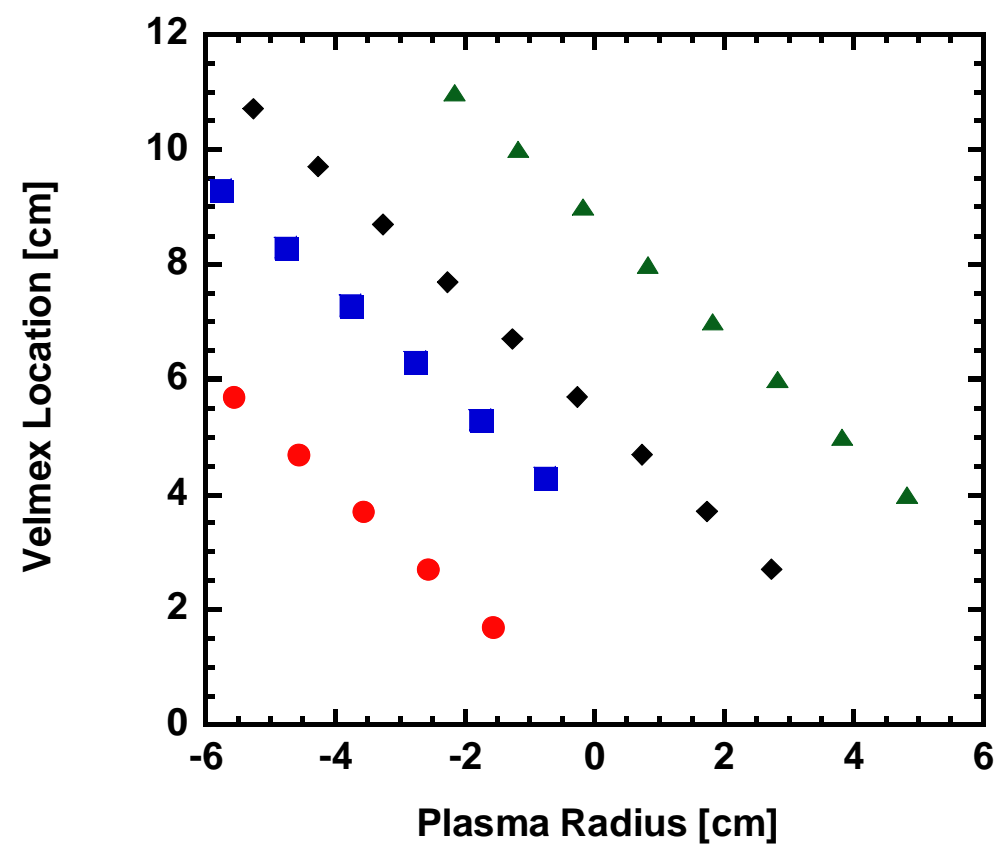

Figure 6.14 Radial plasma coverage of the vacuum collection mirror for scattering angles of $60^{\circ}(\bullet), 70^{\circ}$ $(\square), 80^{\circ}(\bullet)$, and $90^{\circ}(\boldsymbol{\Delta})$.

The collection beam waists were determined by fixing the collection mirror to a known radial location and scattering angle while measuring the signal amplitude as the alignment wedge was moved radially across the vacuum chamber. An example of the self normalized signal amplitudes for a scattering angle of $80^{\circ}$ is shown in Figure 6.15. A summary of the measured collection beam waists for each scattering angle is given in Table 6.3. The measured $1 / e^{2}$ beam waists for the collection optics are comparable to the ZEMAX optical design predictions. Small differences in the measured beam waist for all the scattering angles can arise from several sources, including the surface quality of the alignment wedges and errors in the Gaussian fitting. The large difference in the beam 
waist for the $60^{\circ}$ scattering angle is attributed to the system approaching the line-of-sight limits and the beam being clipped by the internal surfaces of the vacuum chamber.

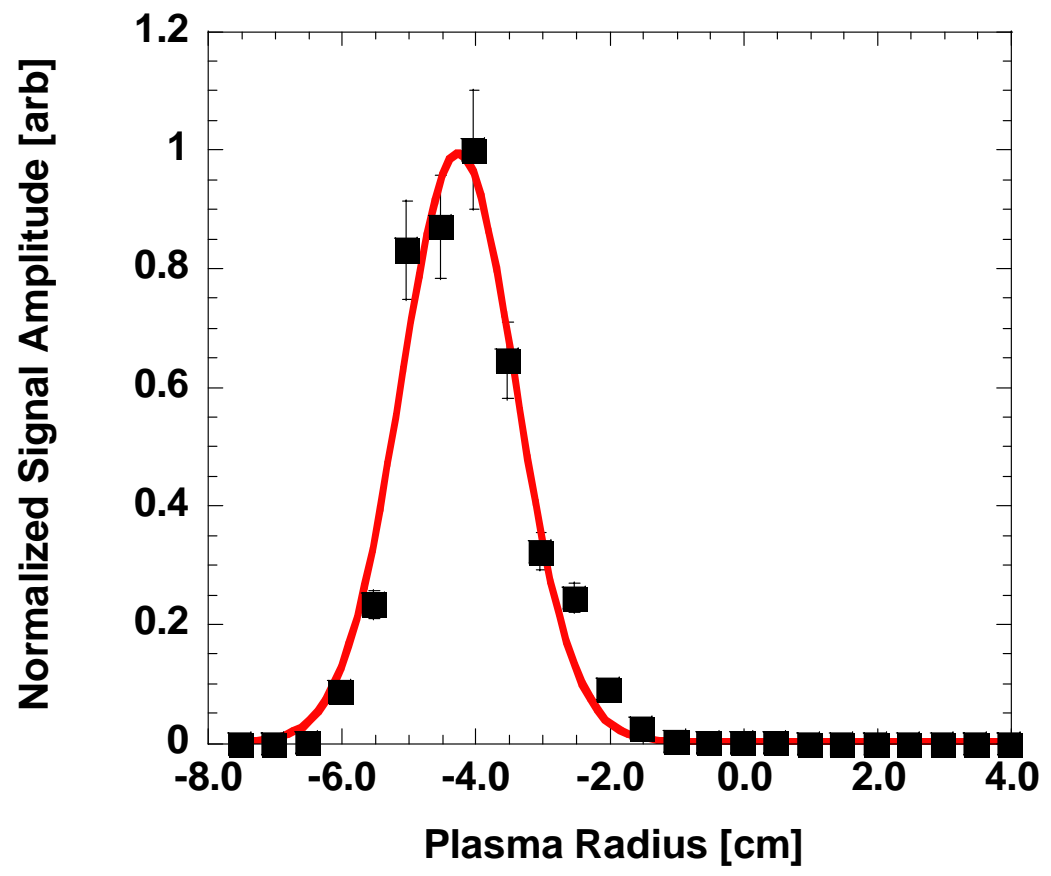

Figure 6.15 Normalized signal amplitude for a scattering angle of $80^{\circ}$ to determine the collection beam waist. A Gaussian fit (_) was used to determine the beam waist.

Table 6.3 Measured and predicted ZEMAX collection beam waists.

\begin{tabular}{|c|c|c|}
\hline $\begin{array}{c}\text { Scattered Angle } \\
{[\mathrm{deg}]}\end{array}$ & $\begin{array}{c}\text { Measured Waist } \\
\left(1 / e^{2} \text { point }\right)[\mathrm{cm}]\end{array}$ & $\begin{array}{c}\text { ZEMAX Predicted } \\
\text { Waist }[\mathrm{cm}]\end{array}$ \\
\hline 90 & $1.34 \pm 0.08$ & 1.83 \\
\hline 80 & $1.72 \pm 0.15$ & 1.83 \\
\hline 70 & $1.54 \pm 0.10$ & 1.83 \\
\hline 60 & $0.94 \pm 0.08$ & 1.83 \\
\hline
\end{tabular}

Determining the collection beam waist of the diagnostic also affords a measurement of the relative efficiency of the vacuum mirror and optics used to collect the scattered radiation (See Figure 6.16). The measured signal for the vacuum mirror at $90^{\circ}$ provides the largest signal, while the signal amplitude dropped to $<5 \%$ of the maximum signal when the mirror was set to $60^{\circ}$. The difference in the signal amplitude for the $70^{\circ}$ and $80^{\circ}$ collection angles can be attributed to differences in the aluminum wedge 
surfaces. As noted previously, when the vacuum mirror is at $60^{\circ}$, the field of view is at the extreme limit of the vacuum collection mirror. To improve the possibility of a successful first measurement with the scattering diagnostic, the vacuum mirror was set to collect at $90^{\circ}$ for the experiments reported here.

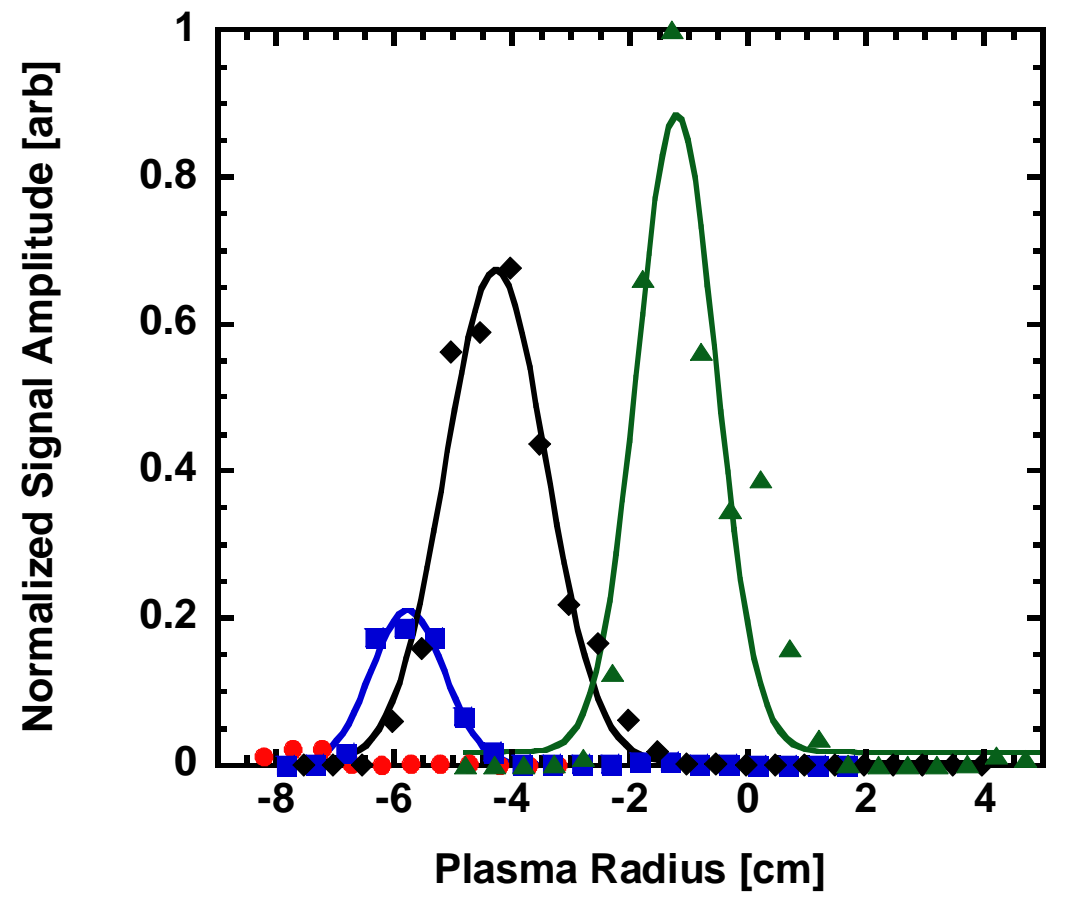

Figure 6.16 Normalized signal amplitude for scattering angles of $60^{\circ}(\bullet), 70^{\circ}(\boldsymbol{\square}), 80^{\circ}(\bullet)$, and $90^{\circ}(\boldsymbol{\Delta})$. The fits are used to determine the beam waists.

\subsubsection{RF Shielding}

Since the detector must operate in an intense rf environment, it is essential to protect the detector as much as possible. The detector is enclosed in a $1 / 4$ inch thick copper box with a battery-powered Analog Modules 322-12 voltage amplifier providing $60 \mathrm{~dB}$ of gain (See Figure 6.17). A copper box and battery powered amplified are used to minimize rf noise pickup. Only the detector's potter horn protrudes from the copper box. SMA and BNC bulkhead connectors are used for the detector's DC bias input and IF 
output. Additionally, a coating of ecosorb AN-72, a microwave absorbing material, is placed on the front plate to minimize any direct reflections.

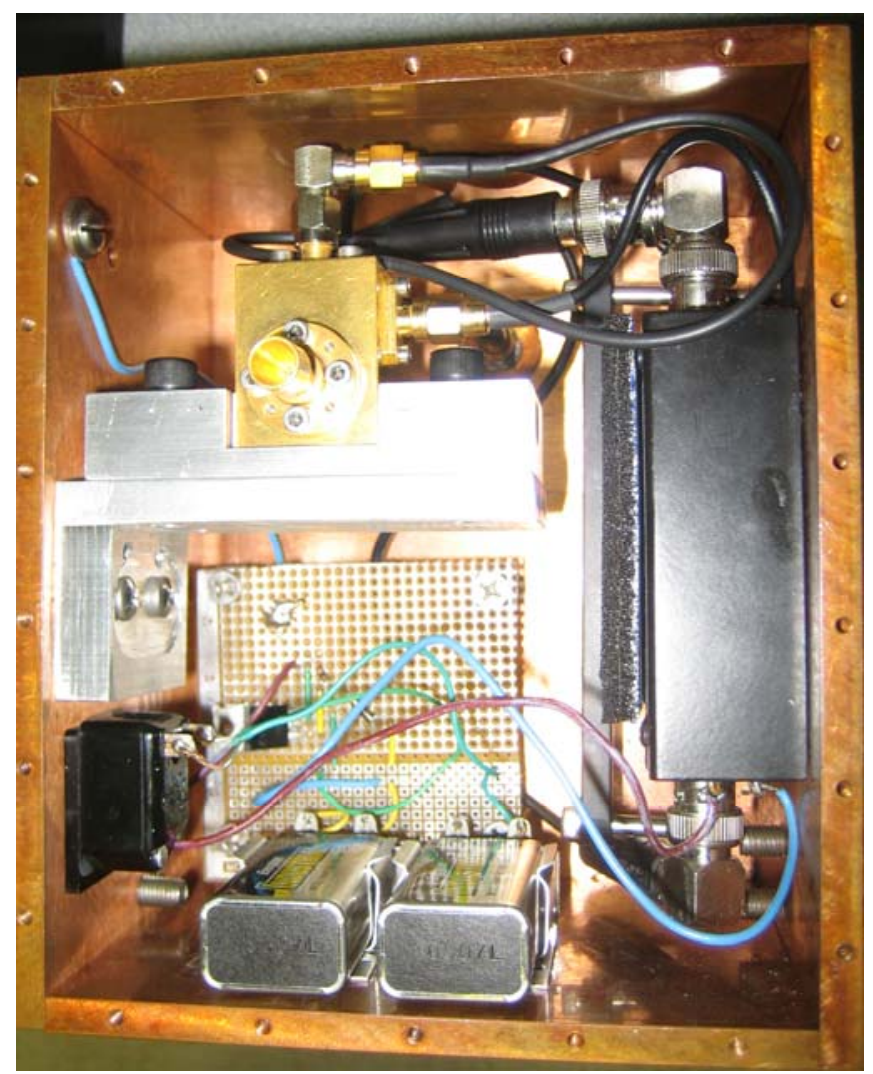

Figure 6.17 Detector housing with the cover removed.

\subsection{Proof-of-Concept Test of the $300 \mathrm{GHz}$ diagnostic}

With the $300 \mathrm{GHz}$ system installed and aligned to the plasma chamber, the first test of the diagnostic was to determine the beam coherence of the system. This test exploits the fact that the diagnostic is essentially an interferometer. The test was accomplished by sending the interaction beam through the plasma chamber via the 90 degree aluminum alignment wedge and vacuum collection mirror while adjusting the length of the reference path length. The reference path length is adjusted with two mirrors mounted on a single linear translation stage. Because the two mirrors are a retro-reflector, the path length change will be twice the linear travel of the stage. As the reference path length is 
changed relative to the interaction path length, an interference pattern with a peak-topeak separation of one half a wavelength is expected. The measured interference pattern for the $300 \mathrm{GHz}$ system is shown in Figure 6.18. As expected, the peak-to-peak spatial separation is approximately $0.5 \mathrm{~mm}$, corresponding to a wavelength of $1 \mathrm{~mm}$, the wavelength of the $300 \mathrm{GHz}$ source. The variation in the signal amplitude is believed to be a result of minor beam misalignments in the reference beam optics, particularly the two mirrors on the linear translation stage.

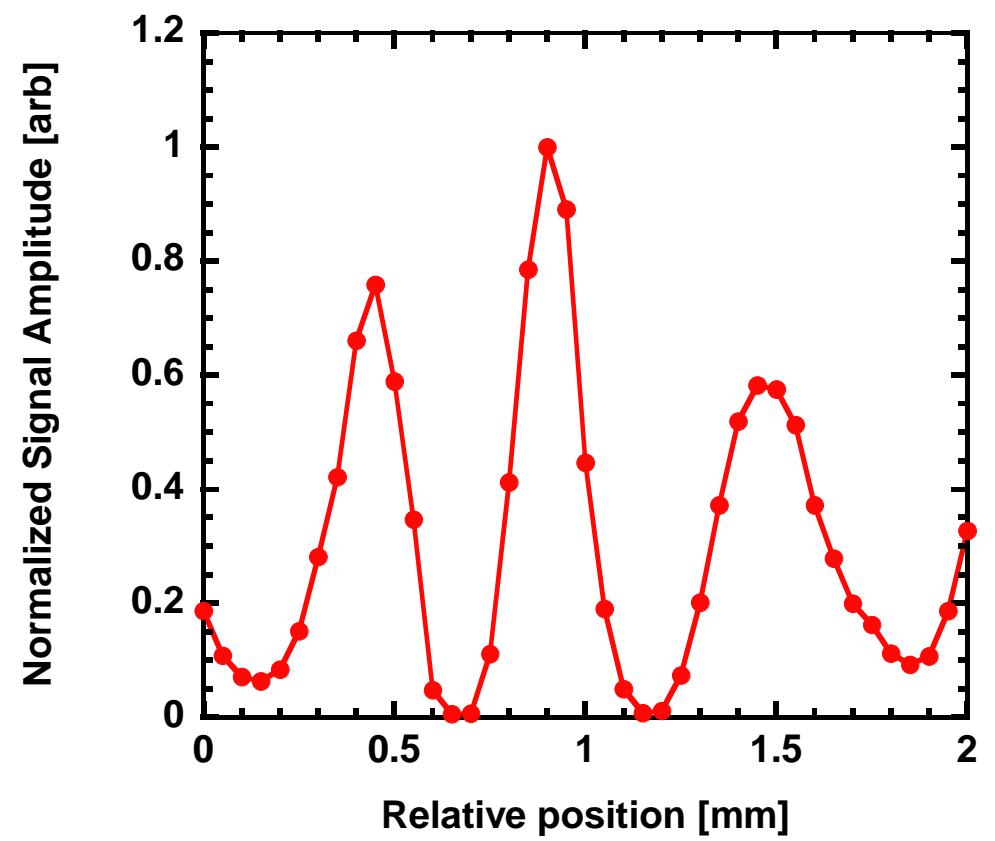

Figure 6.18 Interference pattern of the $300 \mathrm{GHz}$ diagnostic in an interferometer configuration to determine the system coherence.

This measurement revealed one of the principle difficulties in using quasioptical beam propagation compared to a system that employs waveguides or a visible laser based diagnostic: the alignment of the optical components. First, because this system was designed to be portable, several extra optical components were required to keep the optical table compact. With the limited space, placing numerous optical components in close proximity resulted in making alignment measurements problematic in some 
locations. To assist in the alignment process, a permanently mounted laser pointer was employed for much of the optical alignment.

Second, is the size of the beam. With beam waists on the order of 3 inches (6 inch beam diameter) through the majority of the system, the ability to evaluate the beam profile after each component is nearly impossible because of the limited access. The central beam alignment was checked at locations large enough to permit the detector to be placed between optical components, but no complete beam profiles were obtained.

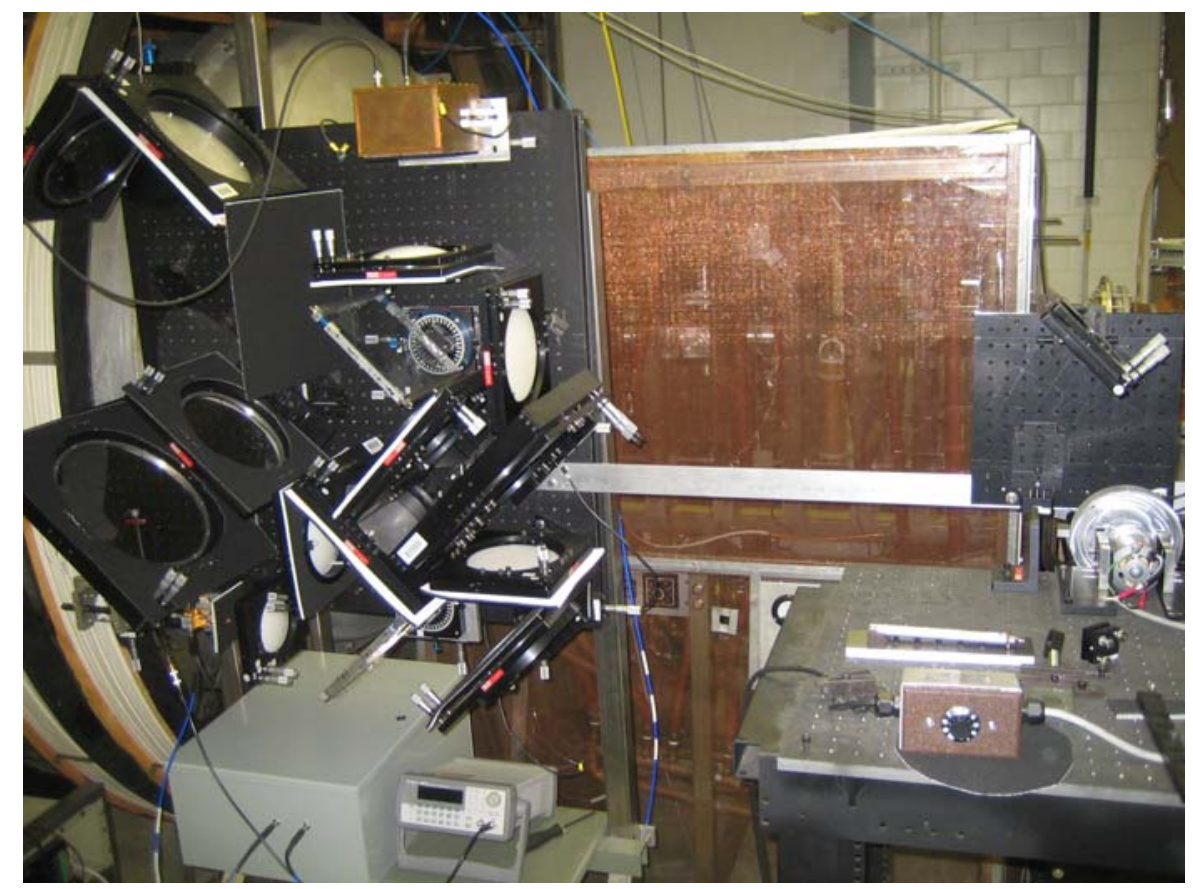

Figure 6.19 Experimental setup for the rotational diffraction grating proof-of-concept test.

To confirm the operation of the detector-mixer as a homodyne detector, a rotating diffraction grating wheel was constructed to test the diagnostic's ability to measure a Doppler shifted signal using homodyne detection before installation on the plasma source. ${ }^{10}$ The optical system was turned away from HELIX to make the test, as shown in Figure 6.19. The aluminum wheel is 6 in. in diameter, with a 1 in. wide face containing 320 triangular teeth separated by $1.49 \mathrm{~mm}$ (see Figure 6.20). Light pulses, from a simple 
laser pointer, passing through a hole in the wheel and detected with a photodiode provide an absolute measurement of the wheel rotation frequency. The rotational frequency of the wheel is variable with a maximum of $133 \mathrm{~Hz}$. Because the wheel is imperfectly balanced and began vibrating above $70 \mathrm{~Hz}$, the rotation frequency was kept below $70 \mathrm{~Hz}$. The Doppler shift from a rotating diffraction grating ${ }^{11}$ is $v_{D}=2 \pi m f_{r} R / d$, where $R$ is the radius of the wheel, $f_{r}$ is the rotational frequency of the wheel, $d$ is the groove spacing, and $m$ is the diffraction order. Figure 6.21 shows the Doppler shifted frequency spectra measured with the mixer for $f_{r}=41$ and $50 \mathrm{~Hz}$. Evident in the signal are diffraction orders $m=1$ and $m=2$ for both wheel frequencies. The signals at 22 and $44 \mathrm{KHz}$ are ambient electronic noise seen in all measurements.

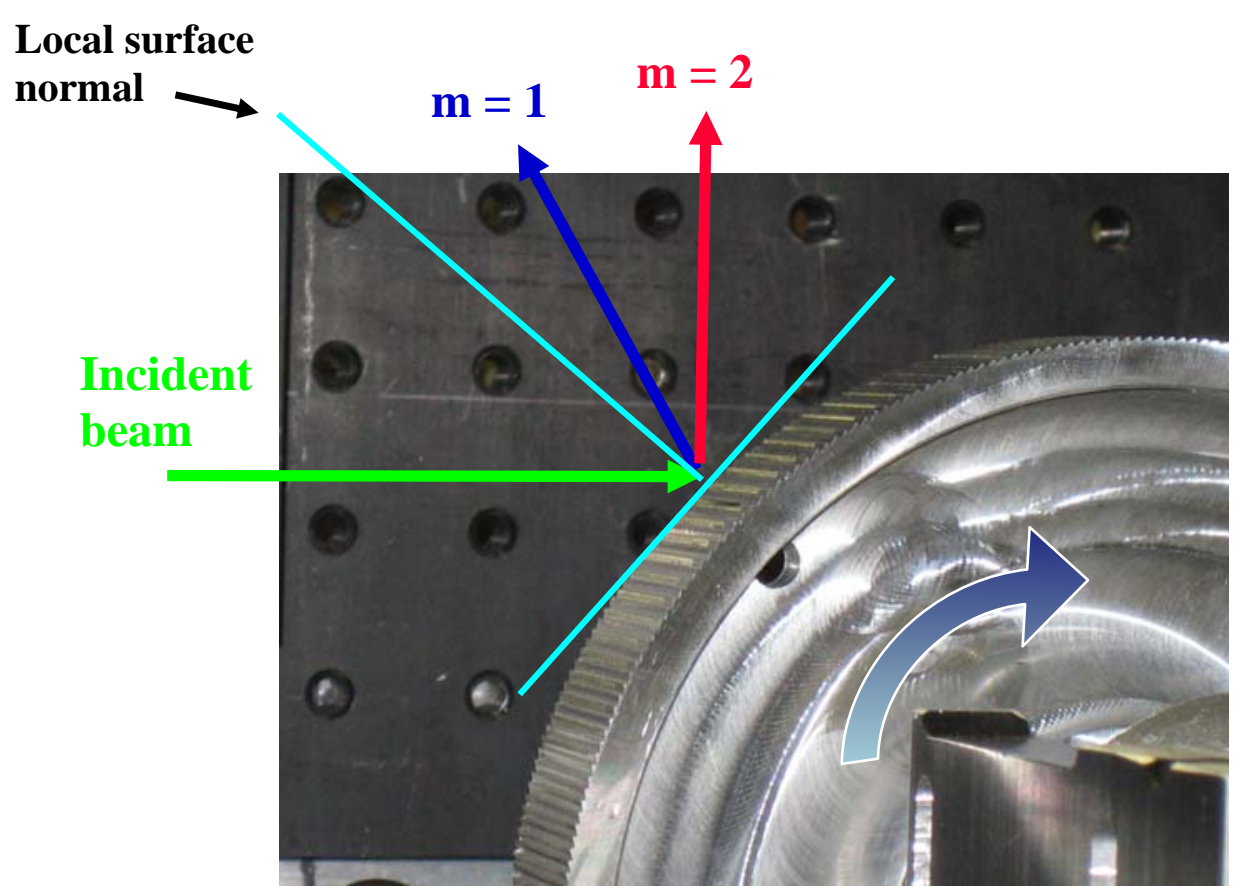

Figure 6.20 Close up of the diffraction wheel with the diffraction geometry overlaid. The hole in the wheel is used to measure the rotational frequency of the wheel. 


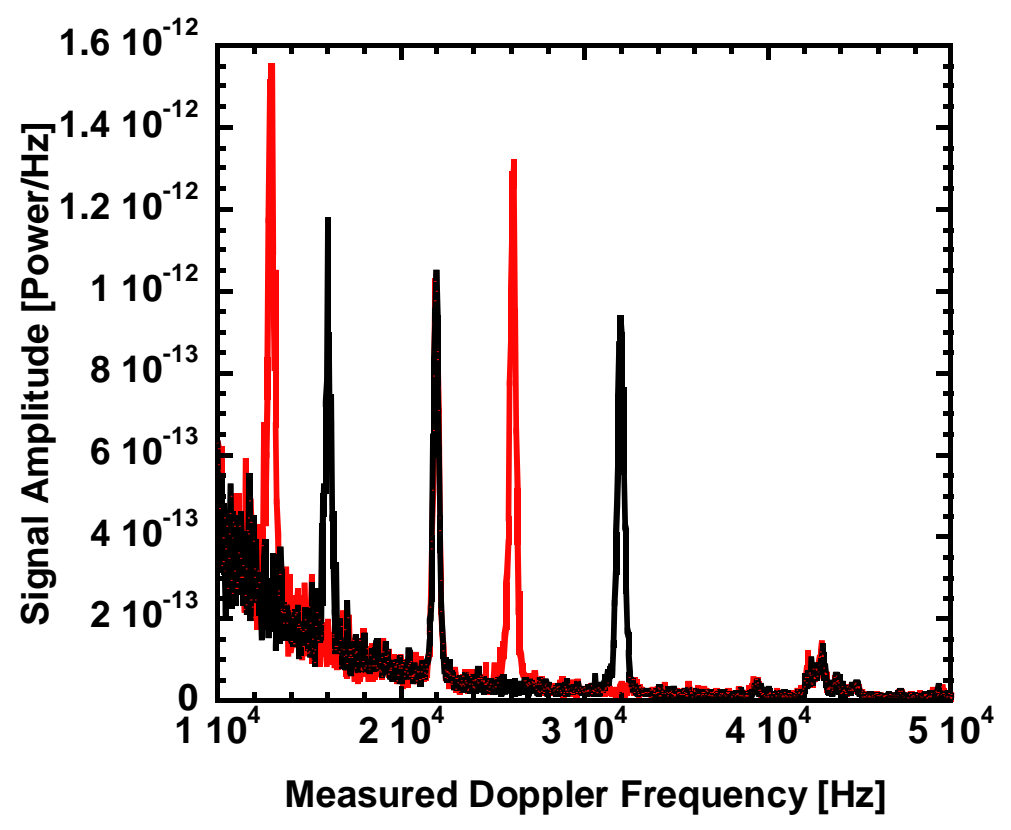

Figure 6.21 Doppler shifted frequency spectra for a wheel rotational frequency of $41 \mathrm{~Hz}$ ( $)$ and 50 $\mathrm{Hz}(\boldsymbol{C})$.

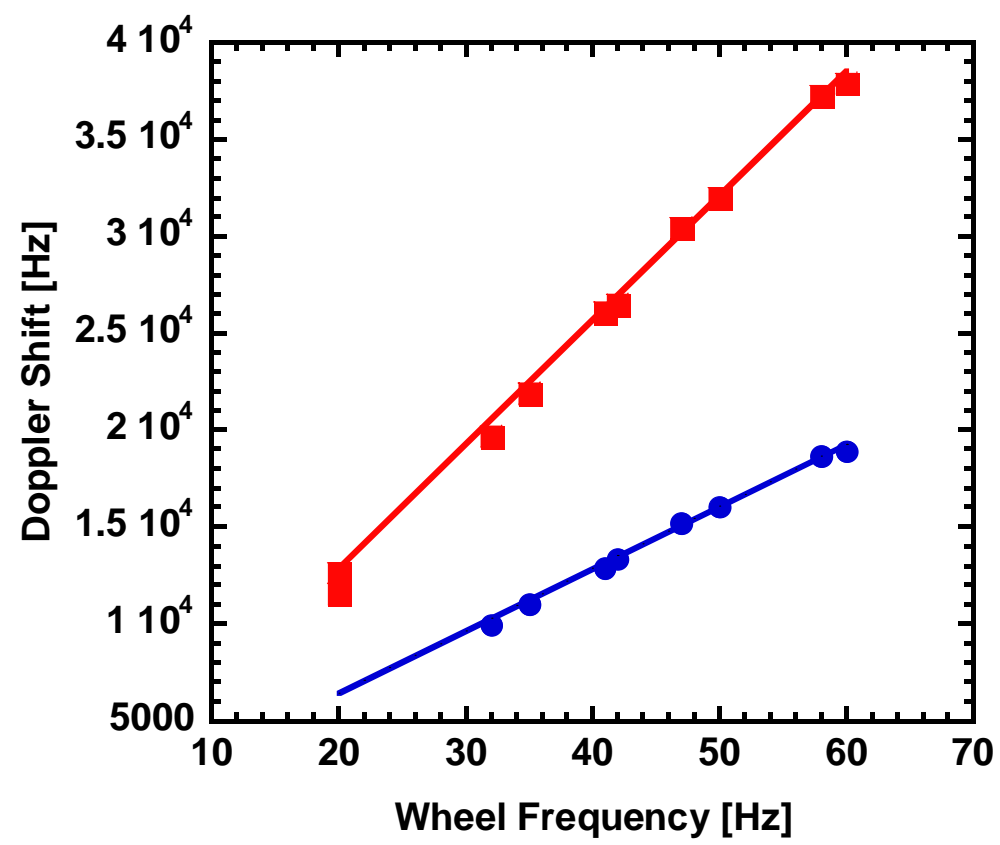

Figure 6.22 Measured and calculated (lines) Doppler shifted frequencies for diffraction orders of $m=1($ and $m=2(\square)$.

The measured and calculated Doppler shifted frequencies for both diffraction orders $(m=1$ and $m=2)$ and all wheel rotational frequencies are shown in Figure 6.22. The data are in excellent agreement with the calculated Doppler frequencies. This test 
verified the ability of the scattering diagnostic to measure Doppler shifted signals in the homodyne detection scheme.

With the CTS system shown to be operating as expected, a test to determine the maximum scattered signal as a function of the sources' DC bias voltage was undertaken for comparison with the quoted output powers in Table 6.1. The measured signal amplitudes were obtained from the peaks of the $m=2$ Doppler shifted signals with the wheel rotational frequency fixed and normalized to the maximum value. The quoted output powers, normalized to the maximum value, and measured normalized signal amplitudes are shown in Figure 6.23. While the quoted source power increases as a function of the bias voltage, the measured signal is largest at a bias voltage of $6 \mathrm{~V}$. Based on this measurement, the DC bias voltage on the $300 \mathrm{GHz}$ source was set to $6 \mathrm{~V}$, to provide the largest amount of power for scattering.

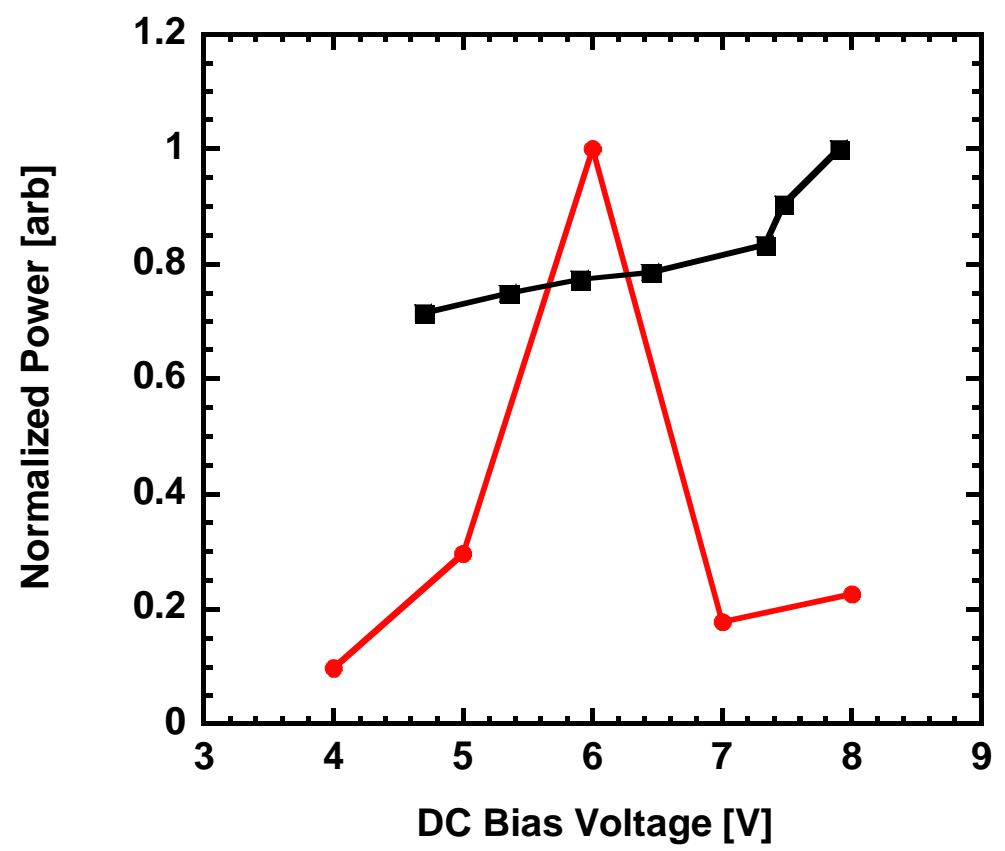

Figure 6.23 Normalized signal amplitude $(\bullet)$ and the normalized $300 \mathrm{GHz}$ source output powers ( $\square$ ) from Table 6.1. 
From these proof-of-concept measurements, a quantitative measurement of the noise floor was also obtained, thus allowing for an estimate of the minimum detectable signal for the diagnostic to be determined. With an available incident beam power $\left(P_{0}\right)$ of approximately $1 \mathrm{~mW}$ in HELIX, incident and collection beam waists $\left(w_{0}\right)$ of $17 \mathrm{~mm}$, and a measured noise floor voltage of approximately $450 \mathrm{nV}$, the minimum scattered power and an expected range of density fluctuation amplitudes for several scattered powers and background densities may be calculated. Given the measured noise floor voltage of 450 $\mathrm{nV}$ and the quoted detector sensitivity of $600 \mathrm{mV} / \mathrm{mW}$, the minimum measureable scattered power is approximately $7.5 \times 10^{-10} \mathrm{~W}$. Recalling that the scattered power from a coherent density fluctuation is $P_{s}=1 / 4 P_{0} r_{e}^{2} \lambda_{0}^{2} L_{v}^{2}(\tilde{n})^{2}$, the density fluctuation amplitude can be determined as a function of the scattered power. After substitution of the numerical quantities $r_{e}=2.81 \times 10^{-12} \mathrm{~mm}, L_{\mathrm{v}}=34 \mathrm{~mm}$, and $\lambda_{0}=1 \mathrm{~mm}$, the resultant equation is $\tilde{n}=6.61 \times 10^{14} \sqrt{P_{s}}$ where $P_{s}$ is in Watts and $\tilde{n}$ is in $\mathrm{cm}^{-3}$. The density fluctuation amplitudes are then normalized to a range of background densities and scattered powers to determine the percentage the density must be fluctuating to produce the corresponding scattered powers. A table of the density fluctuation amplitudes normalized to background densities $\left(n_{0}\right)$ typical in a helicon source for a range of scattered powers is given in Table 6.4.

Table 6.4 Calculated $\tilde{n} / n_{0}$ percentages.

\begin{tabular}{|c|c|c|c|}
\hline$\tilde{n} / n_{0} \times 100 \%$ & $n_{0}=10^{11} \mathrm{~cm}^{-3}$ & $n_{0}=10^{12} \mathrm{~cm}^{-3}$ & $n_{0}=10^{13} \mathrm{~cm}^{-3}$ \\
\hline$P_{s}=10^{-6} \mathrm{~W}$ & 661 & 66.1 & 6.61 \\
\hline$P_{s}=10^{-8} \mathrm{~W}$ & 66.1 & 6.61 & 0.661 \\
\hline$P_{S}=10^{-10} \mathrm{~W}$ & 6.61 & 0.661 & 0.0661 \\
\hline$P_{S}=10^{-12} \mathrm{~W}$ & 0.661 & 0.0661 & 0.00661 \\
\hline
\end{tabular}


Values of $\tilde{n} / n_{0}>10 \%$ are unphysically large and are ignored. For the values where $\tilde{n} / n_{0}<10 \%$, a detectable amount of scattered power $\left(P_{s} \geq 1 \times 10^{-10} \mathrm{~W}\right)$ may be produced for densities greater than $10^{11} \mathrm{~cm}^{-3}$. With measured radial density profiles in the WVU helicon source having peak densities on the order of $10^{13} \mathrm{~cm}^{-3}$ and edge densities of approximately $5 \%$ of the peak $\left(5 \times 10^{11} \mathrm{~cm}^{-3}\right)$, the $300 \mathrm{GHz}$ collective Thomson scattering diagnostic does have the capability to measure electrostatic fluctuations in HELIX. A limitation of this calculation is that it does not take into consideration the propagation direction of the fluctuation or the actual power coupled into the HELIX chamber. The direction of the fluctuation, via the conservation of momentum and the Bragg condition as discussed earlier, is just as important in obtaining a scattering measurement. This calculation only shows that the system meets the detection threshold for fluctuation given the expected range of densities and density fluctuation amplitudes in HELIX. 


\section{Chapter 6 References}

${ }^{1}$ www.microwaves101.com/encyclopedia/waveguidedimensions.cfm

${ }^{2}$ A.R. Kerr, E. Wollack, and N. Horner, Waveguide Flanges for ALMA Instrumentation, ALMA Memo No. $278,(1999)$.

${ }^{3}$ P.F. Goldsmith, Quasioptical Systems: Gaussian Beam Quasioptical Propagation and Applications, (John Wiley, New Jersey, 1998).

${ }^{4}$ P. Vaveliuk, B. Ruiz, and A. Lencina, Opt. Lett., 32, 927 (2007).

${ }^{5}$ C.C. Davis, Lasers and Electro-Optics: Fundamentals and Engineering, (Cambridge University Press, Cambridge, 2000).

${ }^{6}$ P.D. Potter, A NewHorn Antenna with Suppressed Sidelobes and Equal Beamwidths, Jet Propulsion Laboratory Technical Report No. 32-354, Pasdadena, California, (1963).

${ }^{7}$ M. Krämer, B. Clarenbach, and W. Kaiser, Plasma Sources Sci. Technol., 15, 332 (2006).

${ }^{8}$ RPG Radiometer Physics GmbH, 10 Birkenmaarstraße, Mechenheim, 53340, Germany +49-(0)222599981-0

${ }^{9}$ E.D. Palik, ed., Handbook of Optical Constants of Solids II, (Academic Press, 1991).

${ }^{10}$ D. Veron, "Submillimeter interferometry of high density plasmas," in Infrared and Millimeter Waves, K.J. Button, ed. (Academic, New York, 1979), Vol. 2, pp. 69-135.

${ }^{11}$ J. Howard, Appl. Opt. 31, 1419 (1992). 


\section{Chapter 7: Electrostatic Wave Measurements}

Having successfully tested the homodyne detection method with the rotating grating and validated the optical path with the test targets and the modulated source, the CTS system was fully installed on HELIX and the search begun for the elusive slow wave at plasma parameters similar to those that produced the edge ion heating in Kline et al. ${ }^{1}$

\subsection{Experimental Conditions}

Because the primary motivation of this work is to measure short wavelength fluctuations for the same plasma conditions at which enhanced ion heating was observed in previous HELIX experiments, the plasma parameters for conditions similar to those in Ref. [1] were reexamined in light of the recent modifications to HELIX. The particular rf frequency and magnetic field strength combinations used in these are experiments are indicated by black diamonds on Figure 7.1. Also shown in Figure 7.1 are the perpendicular wave numbers as a function of $\mathrm{rf}$ frequency and magnetic field strength predicted by the slow wave model described in Chapter 4 for a neutral pressure of 8 mTorr, a perpendicular ion temperature of $0.2 \mathrm{eV}$, and for the average of the radial density profiles shown in Figure 7.2 (measured at location 7 in Figure 2.2). The axial ( $r=$ $0)$ and edge $(r=5.5)$ lower hybrid frequencies are shown in Figure 7.1 as black curves to indicate the conditions at which the lower hybrid resonance would appear in the plasma at those radial locations. Comparison with Figure 1.7 (presented in the previous HELIX study) reveals that the region of largest normalized perpendicular wave numbers for these parameters passes through the center of the rf frequency and magnetic field range of the previous experiment. The differences in the rf frequencies and magnetic field strengths 
predicted to be associated with the largest perpendicular wave numbers is due to the increased peak densities in these experiments (a result of using the new gas inlet at the center of HELIX).
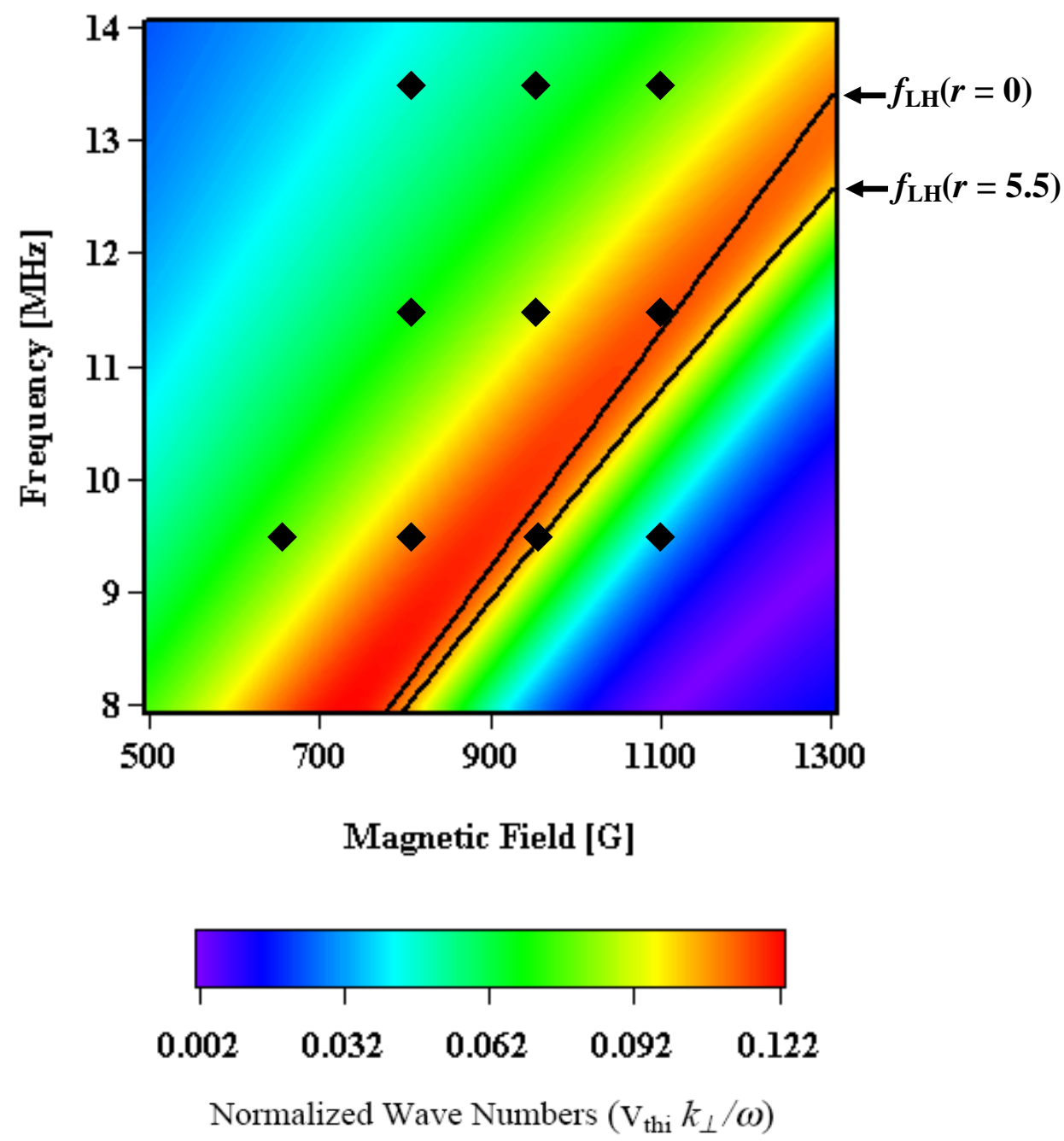

Figure 7.1 Normalized perpendicular wave numbers calculated as a function of rf frequency and magnetic field for a neutral pressure of $8 \mathrm{mTorr}$, ion temperature of $0.2 \mathrm{eV}$, and radial density profile discussed in Chapter 4.2. The lines indicate the lower hybrid frequency on axis $(r=0 \mathrm{~cm})$ and at the plasma edge $(r=$ $5.5 \mathrm{~cm}$ ), while the black diamonds indicate the particular $\mathrm{rf}$ frequencies and magnetic fields that were investigated.

Note also that the band where the normalized wave numbers are largest, corresponding to where the ions would be expected to be heated, extends beyond the region bounded by the axial and edge lower hybrid frequencies. The expanded ion heating region is also a product of the larger peak plasma densities (see Figure 4.2, 4.3 
and 4.5). Because the specific experimental parameters of these experiments (the black diamonds in Figure 7.1) extend throughout the band of large normalized wave numbers, any observation of short wavelength fluctuations at the rf frequency with the electrostatic double probe or CTS system at these parameters would be consistent with excitation and damping of the slow wave near the lower hybrid resonance.
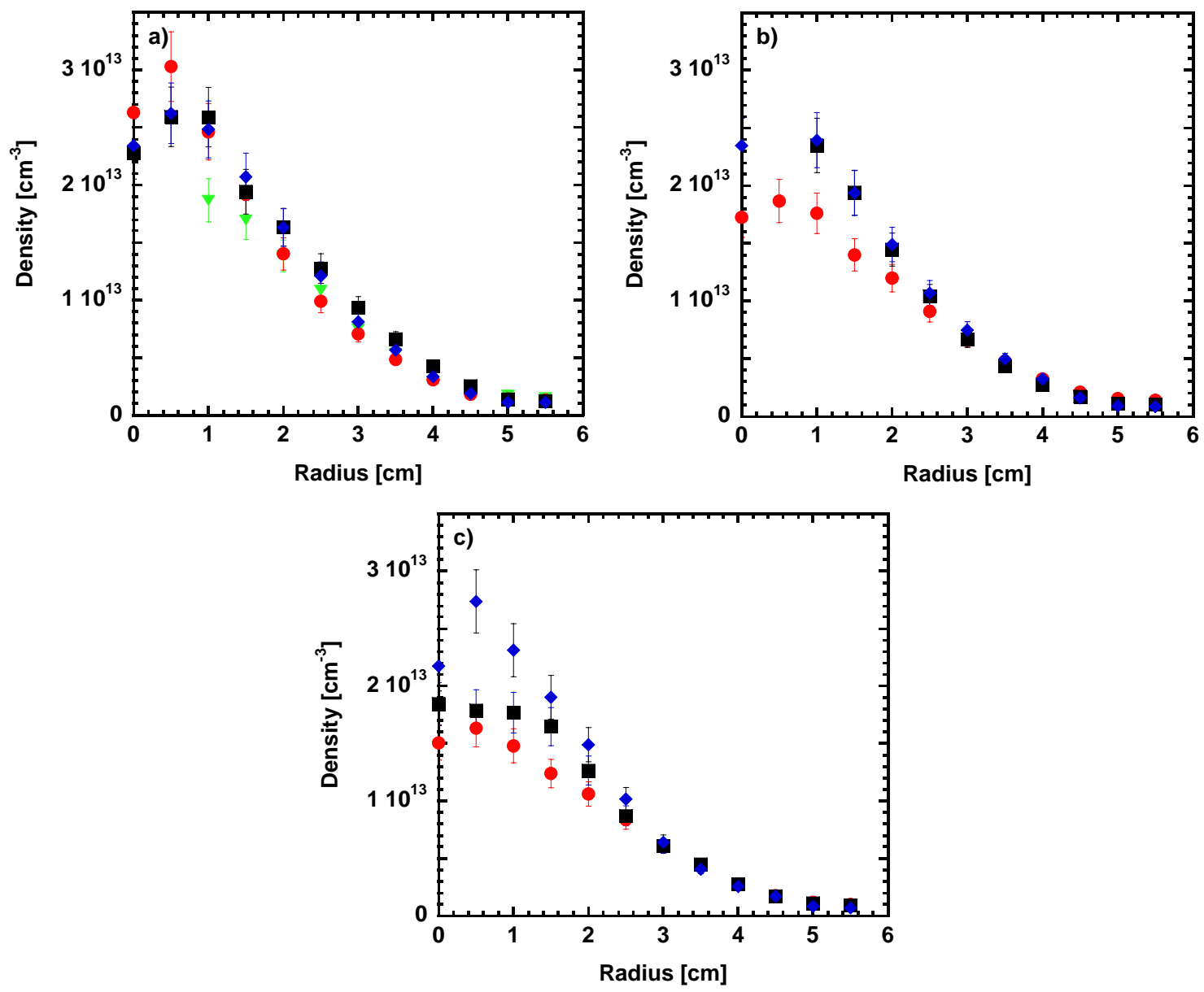

Figure 7.2 Radial density profiles for rf frequencies of (a) $9.5 \mathrm{MHz}$ (b) $11.5 \mathrm{MHz}$ (c) $13.5 \mathrm{MHz}$ at magnetic fields of $(\nabla) 650 \mathrm{G},(\bullet) 800 \mathrm{G},(\boldsymbol{\nabla}) 950 \mathrm{G}$, and $(\diamond) 1100 \mathrm{G}$.

The radial density profiles for four different magnetic field strengths for three different $\mathrm{rf}$ frequencies are shown in Figure 7.2. For all $\mathrm{rf}$ frequencies and magnetic field strengths, the density profiles follow the typical radial profile observed in helicon plasma 
sources operating in the "helicon mode".,2,4 Generally the peak axial densities $(r=0 \mathrm{~cm})$ ranged from $1.5-3 \times 10^{13} \mathrm{~cm}^{-3}$ with densities of $\sim 1 \times 10^{12} \mathrm{~cm}^{-3}$ at the edge $(r=5.5 \mathrm{~cm})$.
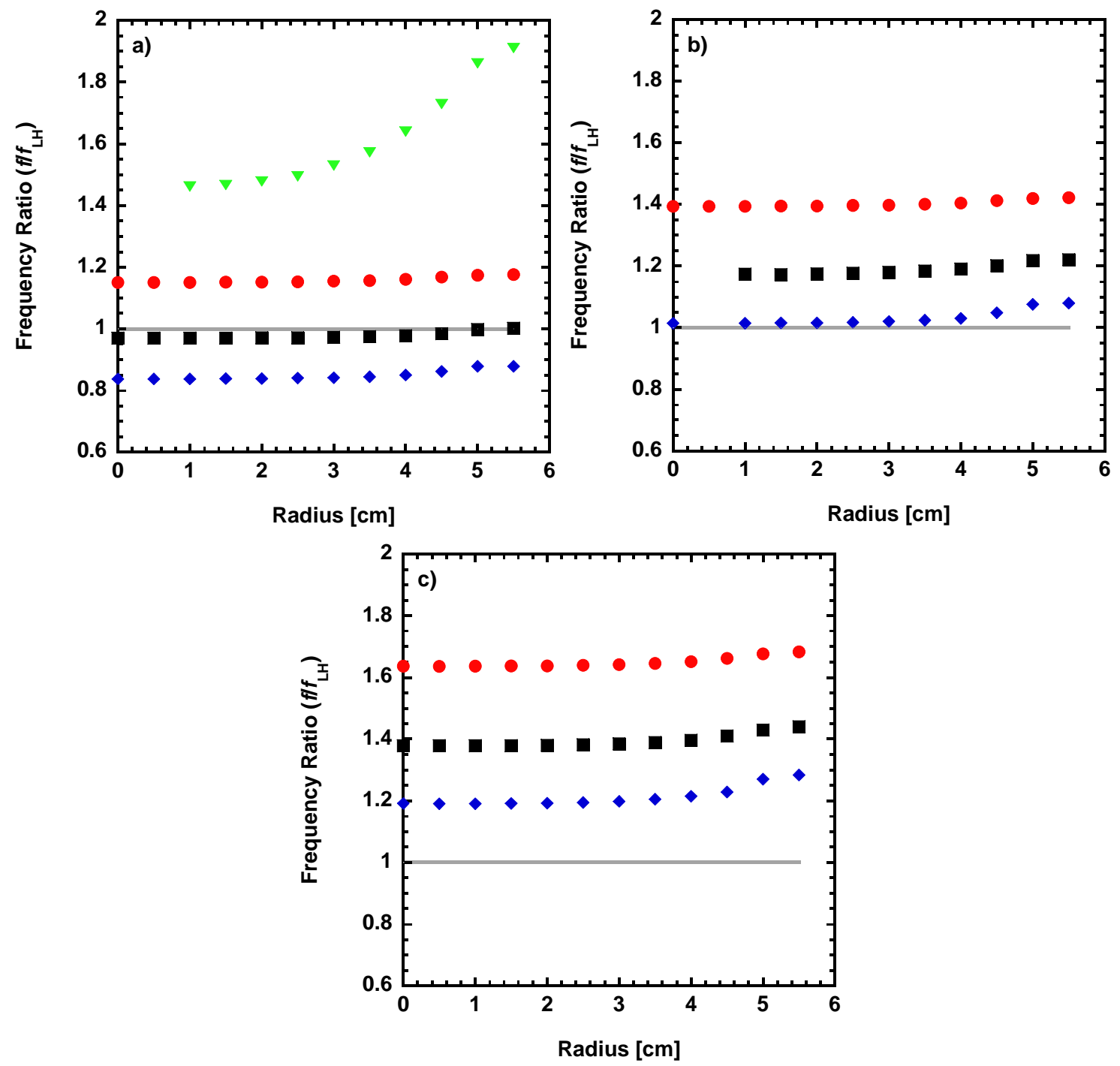

Figure 7.3 Ratios of the $\mathrm{rf}$ frequency to the lower hybrid frequency $\left(f / f_{\mathrm{LH}}\right)$, calculated by the density profiles in Figure 7.2, for rf frequencies of (a) $9.5 \mathrm{MHz}$ (b) $11.5 \mathrm{MHz}$ (c) $13.5 \mathrm{MHz}$ at magnetic fields of $(\nabla) 650 \mathrm{G},(\bullet) 800 \mathrm{G},(\square) 950 \mathrm{G}$, and $(\diamond) 1100 \mathrm{G}$. The (—) at $f / f_{\mathrm{LH}}=1$ is to highlight the resonance condition.

Based on the measured plasma density, the rf frequency normalized to the local lower hybrid frequency as a function of radius is shown in Figure 7.3 for each of the rf frequencies and magnetic field strengths shown in Figure 7.2. For an rf frequency of 9.5 MHz (Figure 7.3a), the plasma source operates above the lower hybrid resonance 
frequency $\left(f / f_{\mathrm{LH}}>1\right)$ for magnetic field strengths $<800 \mathrm{G}$ and below the lower hybrid resonance frequency $\left(f / f_{\mathrm{LH}}<1\right)$ for magnetic field strengths $>950 \mathrm{G}$. At rf frequencies of 11.5 and $13.5 \mathrm{MHz}$ (Figure 7.3b and 7.3c) the source operates almost entirely in the condition $f / f_{\mathrm{LH}}>1$; except for an $\mathrm{rf}$ frequency of $11.5 \mathrm{MHz}$ and magnetic field strength of 1100 G. The important feature highlighted by these calculations is that the operational range of the HELIX source includes regimes above and below $f / f_{\mathrm{LH}}=1$, which is ideal for investigations of slow wave excitation near the lower hybrid resonance.
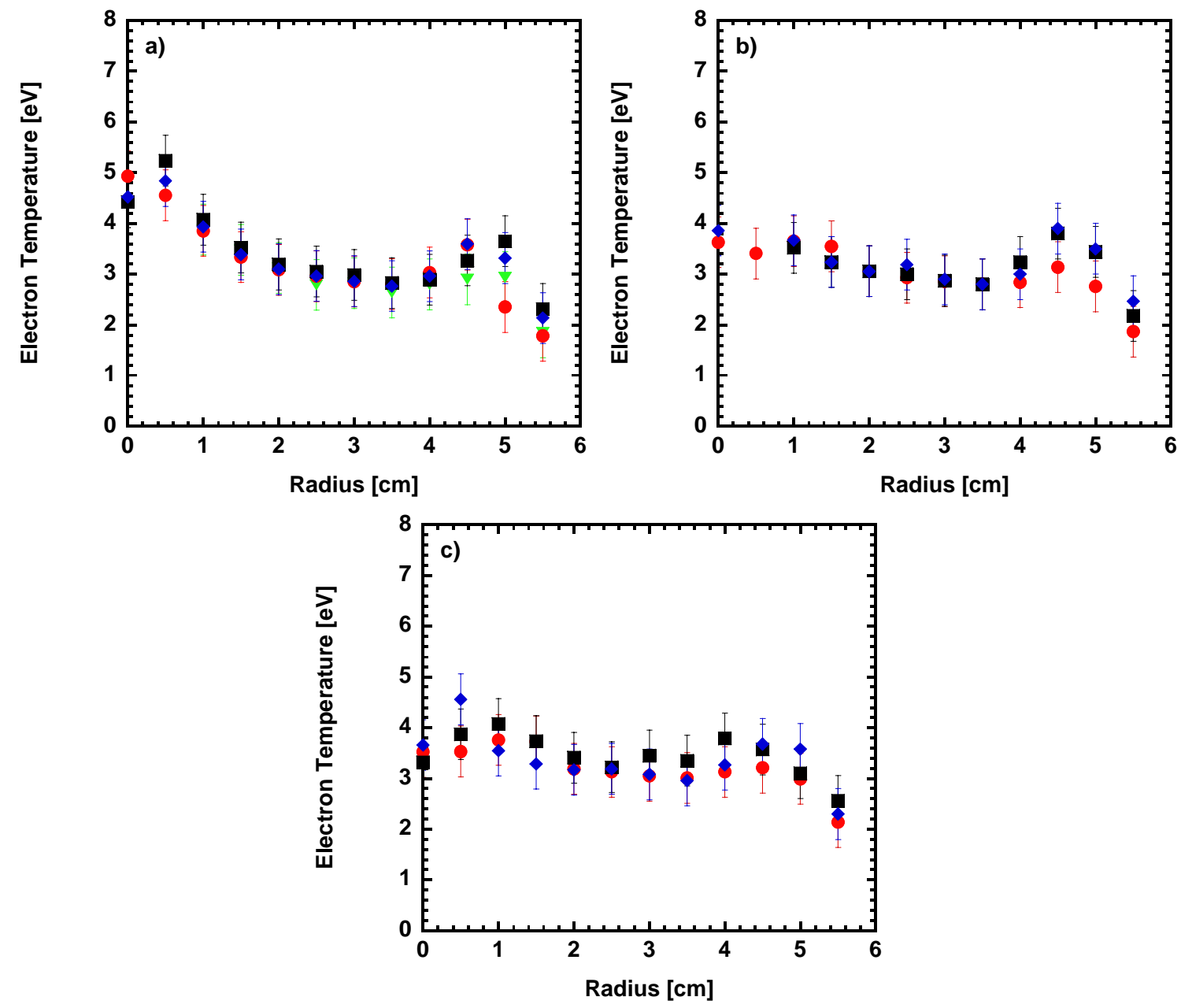

Figure 7.4 Electron temperature radial profiles for rf frequencies of (a) $9.5 \mathrm{MHz}$ (b) $11.5 \mathrm{MHz}$ (c) 13.5 $\mathrm{MHz}$ at magnetic fields of $(\nabla) 650 \mathrm{G}(\bullet) 800 \mathrm{G}(\boldsymbol{\nabla}) 950 \mathrm{G}(\bullet) 1100 \mathrm{G}$.

The final plasma parameters of interest for these investigations are the radial profiles of electron temperature (Figure 7.4) and plasma potential (Figure 7.5). While the 
general trend in both the electron temperature and plasma potential is that of an axially peaked profile, there is an intriguing, radially localized, peak in the region $4<r<5 \mathrm{~cm}$ for almost all the source conditions investigated. Because the calculation for the plasma potential is dependant on the electron temperature, it is no surprise the trends in the plasma potential follow the electron temperature. The most pronounced off-axis peaks in the electron temperature occur for an rf frequency of $11.5 \mathrm{MHz}$ and magnetic field strengths of 950 and 1100 Gauss - which correspond to the conditions expected to yield the largest perpendicular wave numbers as shown in Figure 7.1
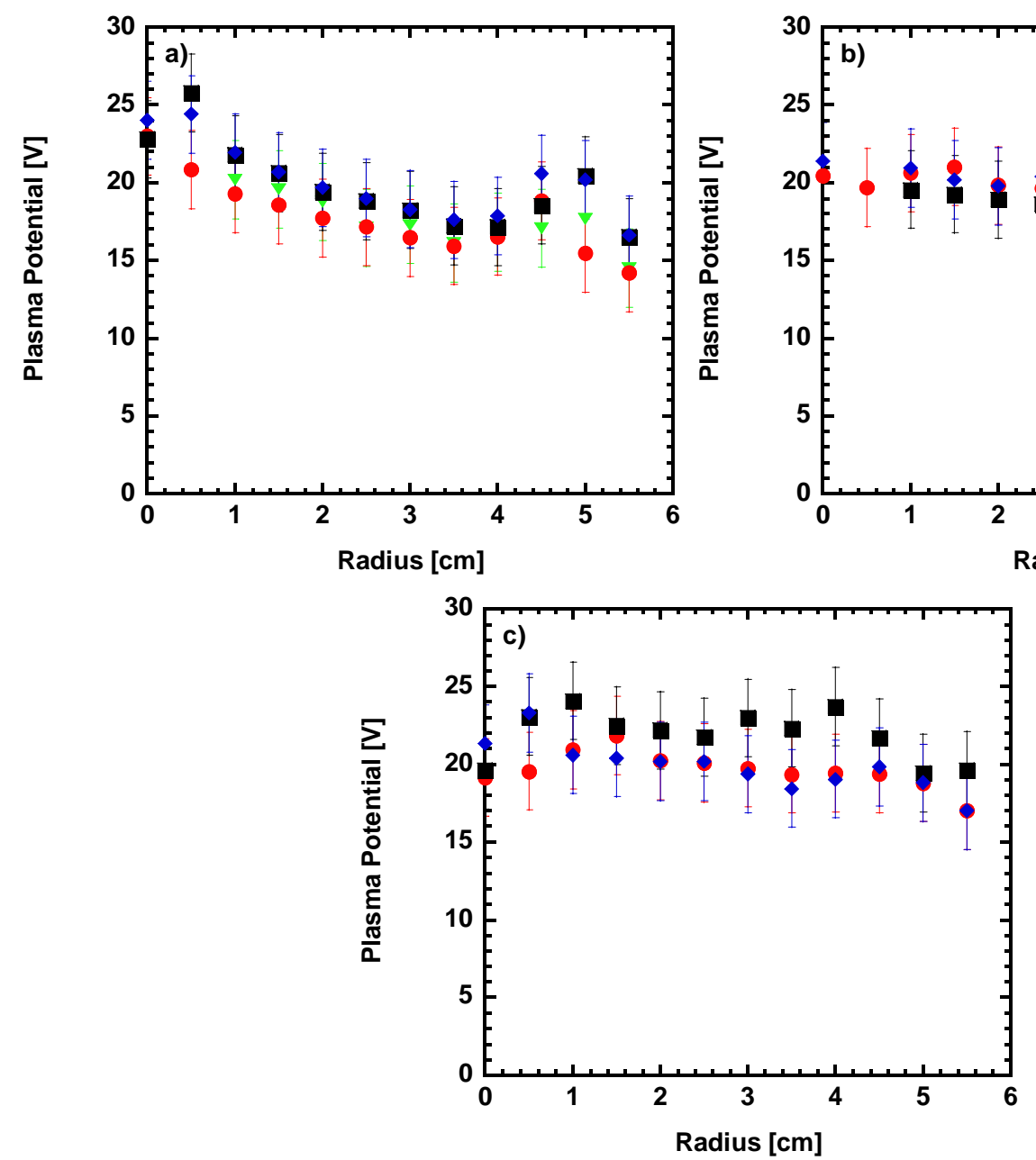

Figure 7.5 Plasma potential radial profiles for rf frequencies of (a) $9.5 \mathrm{MHz}$ (b) $11.5 \mathrm{MHz}$ (c) $13.5 \mathrm{MHz}$ at magnetic fields of $(\nabla) 650 \mathrm{G}(\bullet) 800 \mathrm{G}(\boldsymbol{\nabla}) 950 \mathrm{G}(\bullet) 1100 \mathrm{G}$. 


\subsection{Collective Thomson Scattering Measurements - Part 1}

The first measurements with the $300 \mathrm{GHz}$ CTS diagnostic were obtained for an argon plasma at a neutral pressure of 8 mTorr, magnetic field of 800 Gauss, rf frequency of $9.5 \mathrm{MHz}$, and 500 Watts of rf power. The vacuum mirror was aligned to collected scattered microwaves at $90^{\circ}(k \sim 89 \mathrm{rad} / \mathrm{cm})$ from a radius of $4.5 \mathrm{~cm}$. Signals from the mixer-detector were processed with a spectrum analyzer and recorded on a PC for later analysis. Because the data obtained were real-time Fourier analyzed by the spectrum analyzer, no post acquisition windowing was required. The measured signal amplitudes for the $300 \mathrm{GHz}$ CTS diagnostic operating with the plasma on and off and with the interaction beam line and source blocked and unblocked are shown in Figure 7.6. That the $\sim 2.5 \mathrm{MHz}$ signal evident in Figure 7.6 decreased only slightly when the interaction beam exiting the HDPE vacuum window was blocked with a microwave absorbing material confirmed that the signal was not due to a real plasma fluctuation. The signal could be from noise in the $300 \mathrm{GHz}$ source itself, from rf noise pickup, from an intrinsic noise source in the detector, from reflections in the reference beam, or from reflections from inside the chamber that somehow propagated backwards through the optical path and beat with the source signal. Rf noise pickup in the detector or the source was ruled out by the measurements with the plasma turned off. The last measurements shown in Figure 7.6, obtained while blocking the $300 \mathrm{GHz}$ source, demonstrated that the $\sim 2.5$ $\mathrm{MHz}$ signal is not intrinsic to the detector but is somehow produced by the $300 \mathrm{GHz}$ source. 


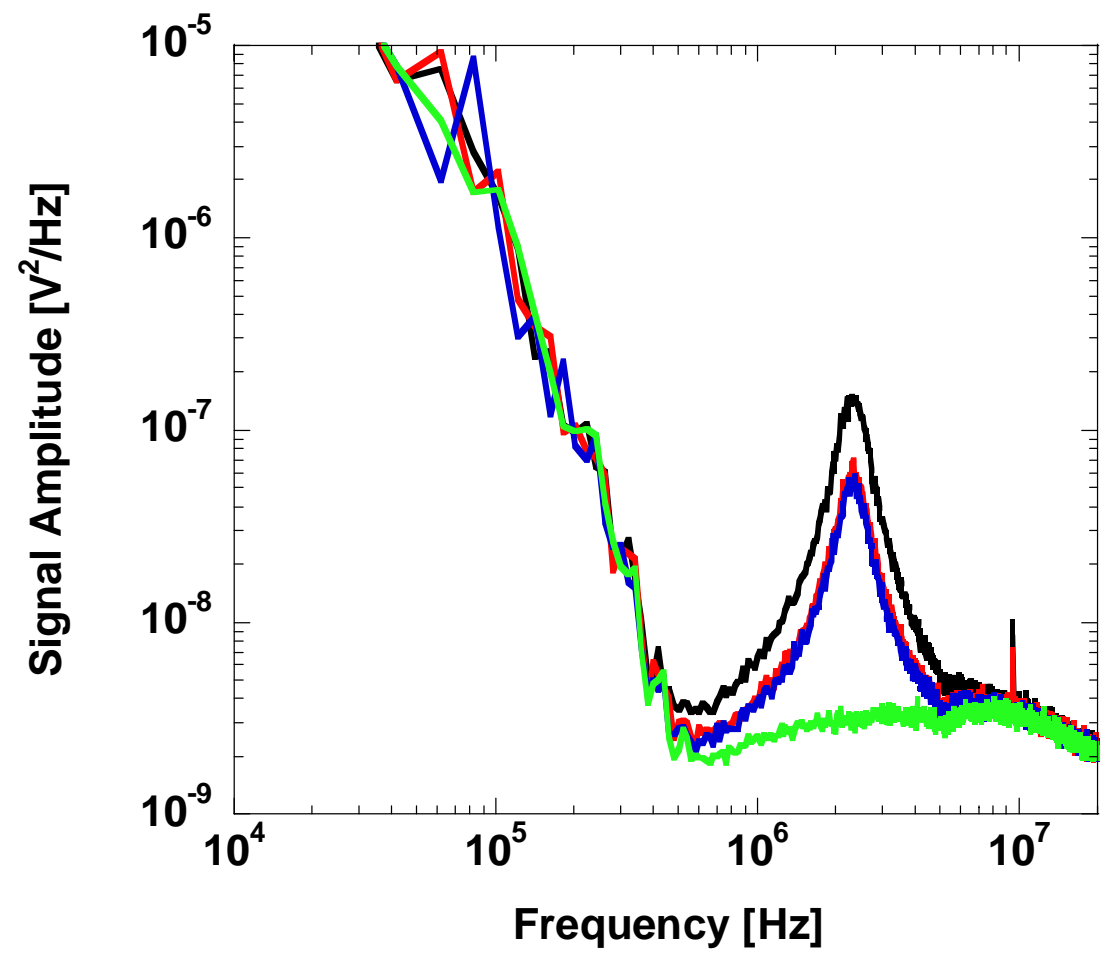

Figure 7.6 Spectrum analyzer signal amplitudes for the $300 \mathrm{GHz}$ diagnostic operational ( $(\boldsymbol{C})$, with the interaction beam line blocked ( $(\boldsymbol{C})$, diagnostic operational with the plasma off ( $(\boldsymbol{C})$, and the $300 \mathrm{GHz}$ source blocked with the plasma off ( $)$.

Since the source frequency had been chirped and measurements limited to frequencies less than $50 \mathrm{kHz}$ in the proof-of-concept tests, this noise signal had not been observed before the full scale experiments. Because the noise signal was large and broad in frequency, it had to be eliminated before the search for plasma fluctuations could progress. To determine if reflections in the reference beam path contributed to the noise signal, the retro-reflector mirror pair was adjusted in steps of $0.1 \mathrm{~mm}$ while monitoring the noise signal with the spectrum analyzer. Measurements were delayed for several seconds after each adjustment step to allow vibrations of the optical table to damp out. Although the noise disappeared briefly each time the mirrors were moved, it invariably returned after a few minutes, suggesting that reflections may be beating back into the source or into the detector. 
The source and detector were removed from the CTS system and placed a few $\mathrm{cm}$ apart to determine if the $2.5 \mathrm{MHz}$ noise signal was intrinsic to the source. Unfortunately the detector was overloaded by the $3 \mathrm{~mW}$ of power emanating from the $300 \mathrm{GHz}$ source and had to be returned to Radiometer Physics in Germany for repair. Several attempts to discuss the noise issue with the company were unsuccessful in obtaining additional information about the noise characteristics of the source. Because the initial experiments did not find any evidence of any plasma fluctuation scattered power, an internal antenna was used to artificially drive waves of sufficient amplitude for detection by the CTS system.

\subsection{Internally Driven Waves}

The disk-exciter, internal antenna was designed to directly excite finite $k_{\perp}$ electrostatic, ion-cyclotron waves propagating perpendicular to the magnetic field. For the magnetic field strengths of HELIX, the wave dispersion of a linear electrostatic ioncyclotron wave is essentially that of an ion-acoustic wave. Externally driven ion-acoustic waves have been used to provide a scattering "target" for use with other collective scattering diagnostics. ${ }^{5,6}$ For a scattering diagnostic with a fixed or limited geometry, such as ours, the limited range of measureable wave numbers may prohibit detection of naturally occurring modes. Additionally, naturally occurring waves may not generate sufficiently large density fluctuations for a detectable scattered signal.

As shown in Figure 7.7, the two-tip electrostatic probe was placed close to the internal antenna and both were placed at a location accessible by the CTS system. The objective was to excite electrostatic ion-cyclotron waves at large perpendicular wave 
numbers, confirm the wave excitation with the electrostatic probe over the measureable wave number range of the probe, and then test the CTS system on the same plasma conditions.

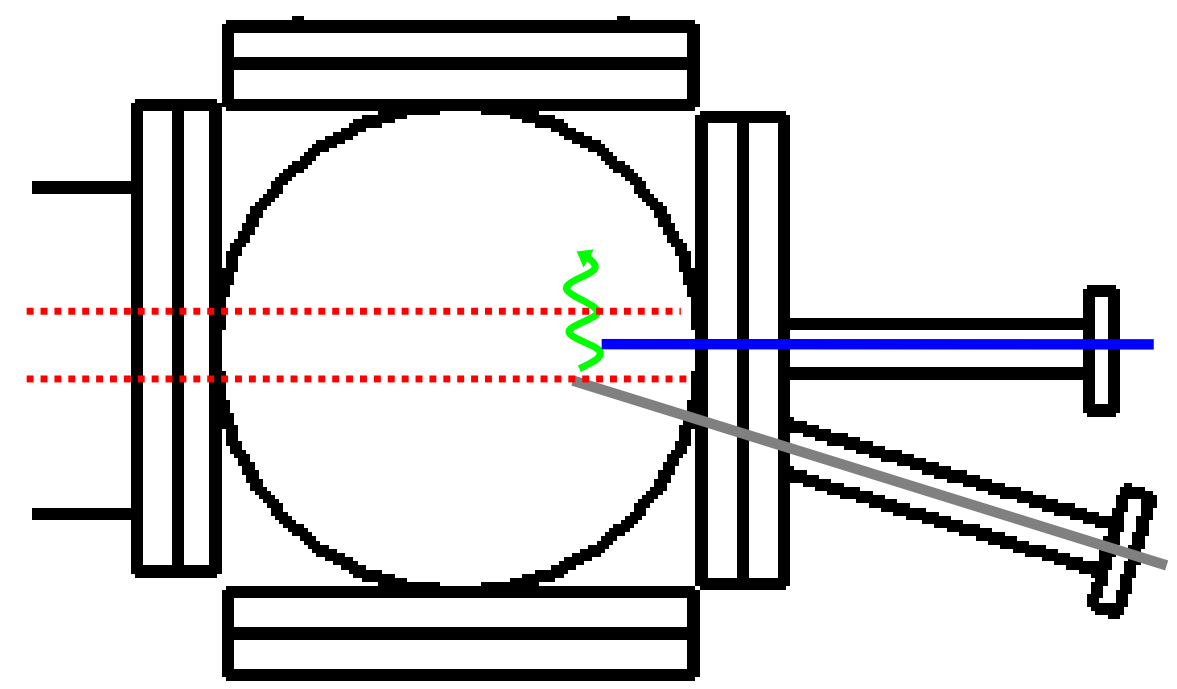

Figure 7.7 Experimental setup for the exciter antenna ( $(-)$ ), electrostatic double probe ( - ), and the interaction beam waist of the scattering diagnostic (......).

Since electrostatic ion-cyclotron waves are essentially perpendicularly propagating ion-acoustic waves for the magnetic fields in HELIX, the rf frequencies chosen for the initial tests were $0.75,1$, and $2 \mathrm{MHz}$. The expected wave numbers for ionacoustic waves $\left(\omega^{2}=k^{2} C_{s}^{2}\right)$ at these frequencies, for an electron temperature of $3 \mathrm{eV}$, are approximately 17,23 , and $47 \mathrm{rad} / \mathrm{cm}$ respectively. These wave numbers are all measureable with the electrostatic double probe. The advantage of exciting waves in the $\sim 2 \mathrm{MHz}$, ion-acoustic, frequency range for these proof-of-principle experiments was that to reach the range of measureable wave numbers $(60$ to $90 \mathrm{rad} / \mathrm{cm}$ ) of the CTS system, the necessary excitation frequencies $(2.5$ to $4 \mathrm{MHz})$ are well away from the typical $\mathrm{rf}$ frequency used for plasma production $(9.5 \mathrm{MHz})$. Therefore, in contrast to looking for scattered wave frequencies at the rf driving frequency, as is done when looking for the 
slow wave, these ion-cyclotron wave scattered signals would be easily distinguished from any rf pickup.

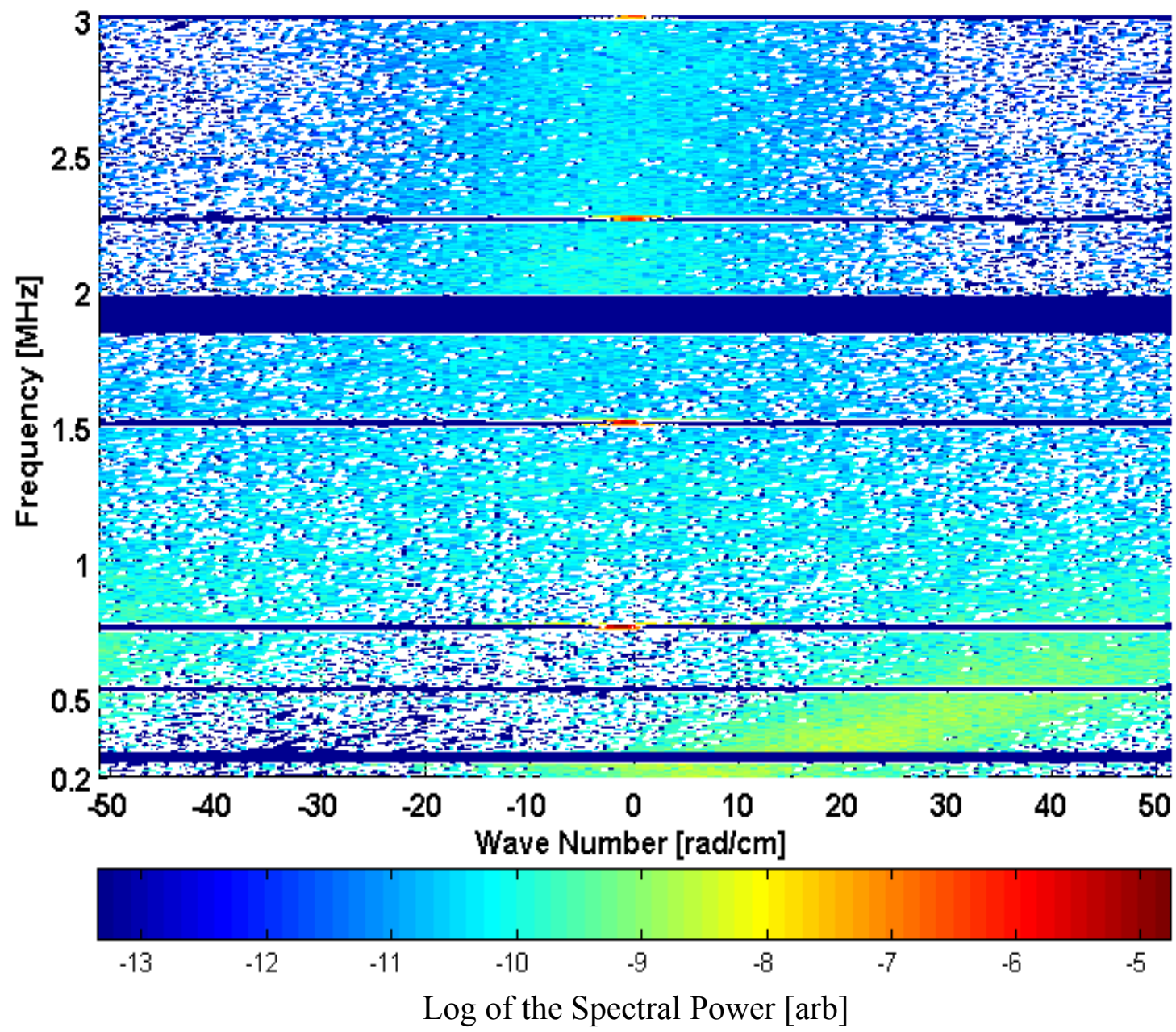

Figure 7.8 Spectral density measured by the electrostatic double probe oriented perpendicular to the magnetic field at $r=4.5 \mathrm{~cm}$ and for the exciter antenna at $r=4.5 \mathrm{~cm}$. The antenna driving frequency was $750 \mathrm{kHz}$.

The experiments to characterize the disk antenna were accomplished in argon plasma with an rf driving frequency of $9.5 \mathrm{MHz}$, an $\mathrm{rf}$ power of $500 \mathrm{~W}$, a neutral pressure of 8 mTorr, and a magnetic field of $800 \mathrm{G}$. With the antenna at a fixed radial location, the electrostatic double probe was stepped radially through the plasma to measure the radial profile of any electrostatic fluctuations. For the first tests of the emitter, the center of the antenna disk was located at a radius of $3 \mathrm{~cm}$ and driven continuously by an amplified 
function generator. The output of the amplifier was maintained at $50 \mathrm{~W}$. Post acquisition spectral density analysis (not shown here) clearly showed the driving frequency and several harmonics, but no waves at finite $k_{\perp}$. It was later determined that during these initial experiments, the antenna head had been destroyed by being placed too close to the high density plasma core. During later experiments, the antenna was kept closer to the plasma edge.

Figure 7.8 shows the spectral density as a function of wave number and frequency for a disk antenna driving frequency of $750 \mathrm{kHz}$. Both the antenna and double probe were located at a radius of $4.5 \mathrm{~cm}$. Only a limited frequency range is shown in Figure 7.8 so that spectrum around the antenna exciter frequency can be carefully examined. The fundamental driving frequency $(750 \mathrm{kHz})$ and the first three harmonics $(1.5,2.25$, and 3 $\mathrm{MHz})$ are all associated with very little phase difference $\left(k_{\perp} \sim 0\right)$ in the spectral density. The spectral densities for driving frequencies of 1 and $2 \mathrm{MHz}$ (not shown) exhibit the same $k_{\perp} \sim 0$ character.

Because it will become relevant when the CTS system frequency spectra measurements are reviewed, it is important to recall that the electrostatic probe measurements were restricted to frequencies greater than $200 \mathrm{kHz}$ because of a strong noise peak at $100 \mathrm{kHz}$ that exists even in the absence of a plasma. Nearly identical noise peaks were reported by Kline in his $\mathrm{PhD}$ dissertation. ${ }^{7}$

The failure to excite any waves with finite $k_{\perp}$ was attributed to a lack of sufficient power coupling to the plasma. With the output power of the amplifier limited to only 50 $\mathrm{W}$ in this frequency range, the currents drawn to the disk antenna may have been too small to exceed the threshold for current driven electrostatic ion cyclotron (CDEIC) wave 
excitation. The excitation threshold for the CDEIC requires that the parallel electron drift velocity must exceed the critical value of the axial component of the wave phase velocity, $v_{D e} \equiv \omega_{R} / k_{\|}$, where $\omega_{R}$ is the electrostatic ion cyclotron frequency $\left(\omega_{R}^{2}=\omega_{c i}^{2}+k_{\perp}^{2} c_{s}^{2}\right){ }^{8}$ Assuming a parallel wave number of $k_{\|} \sim 1.5 \mathrm{rad} / \mathrm{m}$ (based on a wave fitting into the combined chamber length of HELIX and LEIA) and the plasma conditions of a magnetic field strength of 800 Gauss, an electron temperature of $3 \mathrm{eV}$, and $k_{\perp} \sim 5 \mathrm{rad} / \mathrm{cm}$, the calculated wave phase velocity is $\omega_{R} / k_{\|} \sim 10^{6} \mathrm{~m} / \mathrm{s}$. To excite the CDEIC, electrons drawn to the antenna must exceed the phase velocity. The antenna exciter was oriented so that the plane of the disk antenna faced in the azimuthal direction, leaving only the thin edge of the disk available for drawing parallel current to excite the waves. The approximate collecting area of the probe was $\sim 10^{-5} \mathrm{~m}^{2}$. For an output power of $50 \mathrm{~W}$ from the voltage amplifier, corresponding to an output voltage of $50 \mathrm{~V}_{\mathrm{rms}}$, the current drawn to the antenna exciter was $\sim 1$ Amp. Given a local electron density of $\sim 10^{18} \mathrm{~m}^{-3}$, the conservatively estimated upper bound for the electron drift velocity, $\left(v_{D e} \approx I / n q A\right)$, where $I$ is the current, $n$ is the density, $q$ is the elementary charge, and $A$ is the antenna area, is $\sim 5 \times 10^{5} \mathrm{~m} / \mathrm{s}$, well below the CDEIC threshold. Thus, it is not surprising that driven CDEIC waves were not observed.

During the antenna tests, a weak spectral feature between $200 \mathrm{kHz}$ and $1 \mathrm{MHz}$ and with wave numbers well away from $k_{\perp} \sim 0$ was observed for all three antenna driving frequencies. Because the spectral density amplitudes at the antenna excitation frequencies were so large, the finite $k_{\perp}$ features were difficult to distinguish from the background level. Therefore, a series of probe measurements were made without the internal antenna. 
The spectral density at the same radial location and plasma parameters but with the internal antenna removed is shown in Figure 7.9 for frequencies above $200 \mathrm{kHz}$.

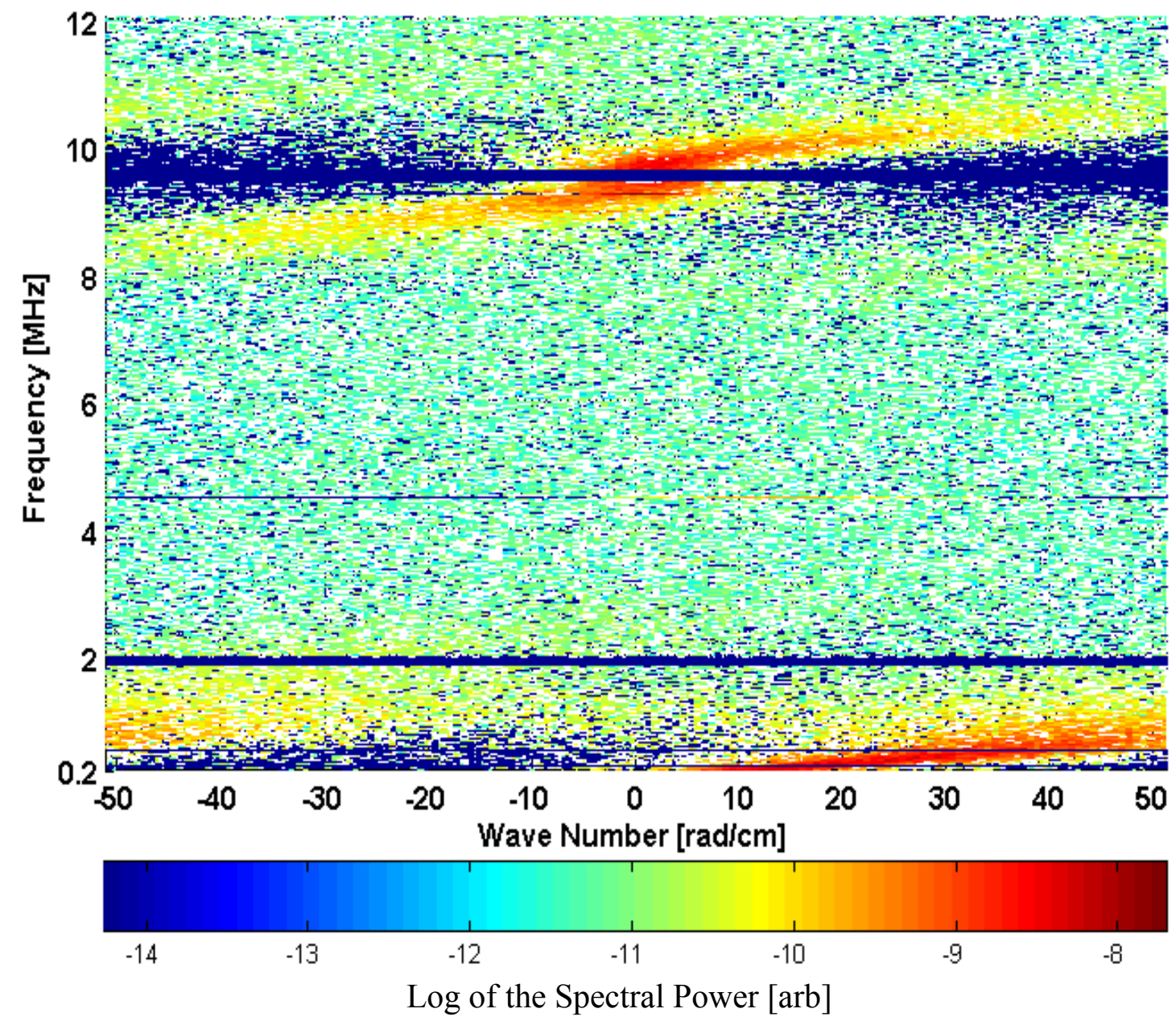

Figure 7.9 Spectral density from the electrostatic double probe oriented perpendicular to the magnetic field at $r=4.5 \mathrm{~cm}$ for a magnetic field of $800 \mathrm{G}$, neutral pressure of $8 \mathrm{mTorr}$, and rf driving frequency of 9.5 MHz. A suppression window at $9.5 \pm 0.1 \mathrm{MHz}$ is applied to assist in identification of the fluctuations.

There are three dominant spectral features in Figure 7.9 in the frequency ranges; $200 \mathrm{kHz}$ to $2 \mathrm{MHz}$ (low frequency - LF), 8 to $9.5 \mathrm{MHz}$ (low side band - LSB), and 9.5 $\mathrm{MHz}$ to $11 \mathrm{MHz}$ (high side band - HSB). Examining only the magnitude of the wave numbers for the moment, the most important detail is that all three features include fluctuations with wave numbers approaching the probe resolution limit of $\sim 51 \mathrm{rad} / \mathrm{cm}$. In fact, particularly for the LF wave, the wave numbers continue beyond the limit and phase 
wrap (due to the limit of $\pm \pi$ in the phase calculation) to the negative wave number side. While detailed wave classification is deferred until after measurements for a range of plasma parameters are presented, there are two important features in this initial measurement that are worth highlighting. First, the perpendicular wave numbers for the fluctuations are approaching the measureable range for the scattering diagnostic, at least for these parameters. Second, there is a striking similarity between the spectral features measured in HELIX with the electrostatic probe and those shown in Figure 1.10 (as measured with a CTS diagnostic in another helicon source). Note also that the enhanced scattering data reproduced in Figure 1.10 indicate measureable fluctuation power at large perpendicular wave numbers and frequencies down to a few hundred $\mathrm{kHz}$.

Although no electromagnetic fluctuation measurements are made in this work to offer direct evidence that these fluctuations are purely electrostatic, the similarity to previous measurements in HELIX ${ }^{9}$ and the recent work of Kramer et al. ${ }^{10}$ strongly suggest that these fluctuations are purely electrostatic and are dominated by fluctuations in the electric potential and not in the electron temperature.

\subsection{Characteristics of the Spontaneously Excited Fluctuations}

In preparation for CTS system measurements of the spontaneously excited

electrostatic fluctuations, detailed measurements of the fluctuations with the two-tip probe were performed for the entire range of experimental conditions. The first aspect of the fluctuations investigated was their direction of propagation. The data shown in Figure 7.9 established a significant perpendicular wave number associated with the fluctuations. Similar data obtained with the probe tips aligned along the magnetic field are shown in 
Figure 7.10. These data are representative of all the measurements obtained for parallel orientation of the probe tips; no significant spectral features at finite parallel wave numbers are observed. For these particular plasma conditions, there is a weak spectral feature at $\sim 1 \mathrm{MHz}$ that extends to parallel wave numbers of $\sim 10 \mathrm{rad} / \mathrm{cm}$. However, in general, the observed fluctuations have no component of propagation along the background magnetic field; consistent with expectations for electrostatic ion cyclotron waves in a plasma with a large ion to electron mass ratio.

Given that the measured perpendicular wave numbers extend up to the limit of the probe wave number range, the maximum wave numbers associated with the fluctuations cannot be uniquely determined from the measurements shown in Figure 7.9. Because the fluctuations are propagating purely perpendicular to the magnetic field, the electrostatic probe can be rotated with respect to the magnetic field direction to reduce the effective separation of the probe tips and thereby increase the maximum measureable wave number. At an angle of $45^{\circ}$ relative to the axis of the source, the effective probe tip separation is approximately $0.043 \mathrm{~cm}$ and the maximum of the measureable perpendicular wave number range increases to $\pm 72.8 \mathrm{rad} / \mathrm{cm}$. The measureable wave number range can be further extended by noticing that in the fluctuation data shown in Figure 7.9 clearly continue past the $\pm \pi$ phase difference boundary and reappear with a $\pi$ offset on the other side of the figure. For fluctuations that are so broad in wave number space that the $\pi$ phase shift due to phase "wrapping" can be clearly identified, the "wrapped" portion of the spectrum can be simply cut and pasted onto the correct end of the wave number spectrum; thereby extending the effective wave number range of the measurement. 


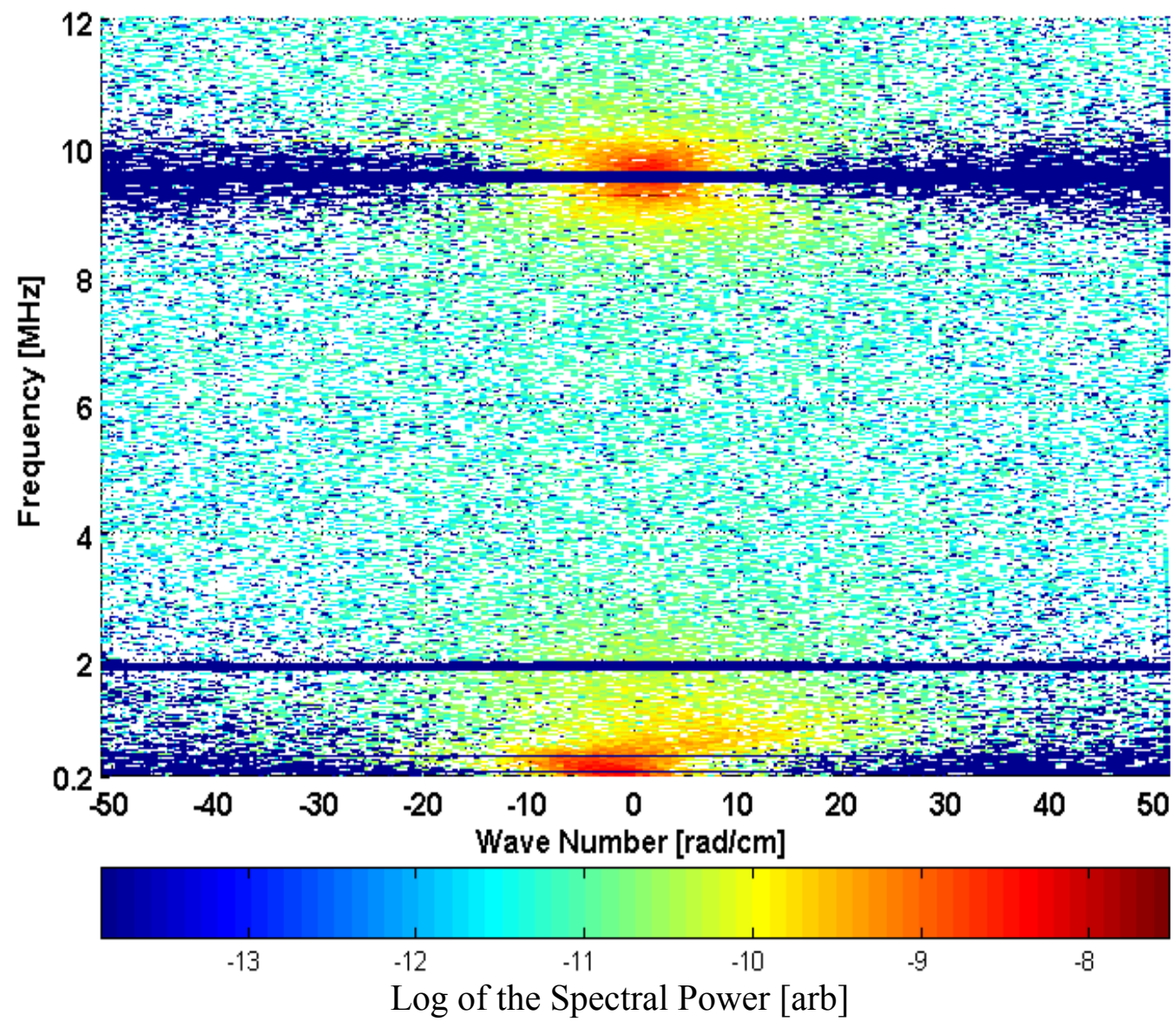

Figure 7.10 Spectral density from the electrostatic double probe oriented parallel to the magnetic field at $r$ $=4.5 \mathrm{~cm}$ for a magnetic field of $800 \mathrm{G}$, neutral pressure of $8 \mathrm{mTorr}$, and rf driving frequency of $9.5 \mathrm{MHz}$. A suppression window at $9.5 \pm 0.1 \mathrm{MHz}$ is applied to enhance the appearance of the fluctuations. 


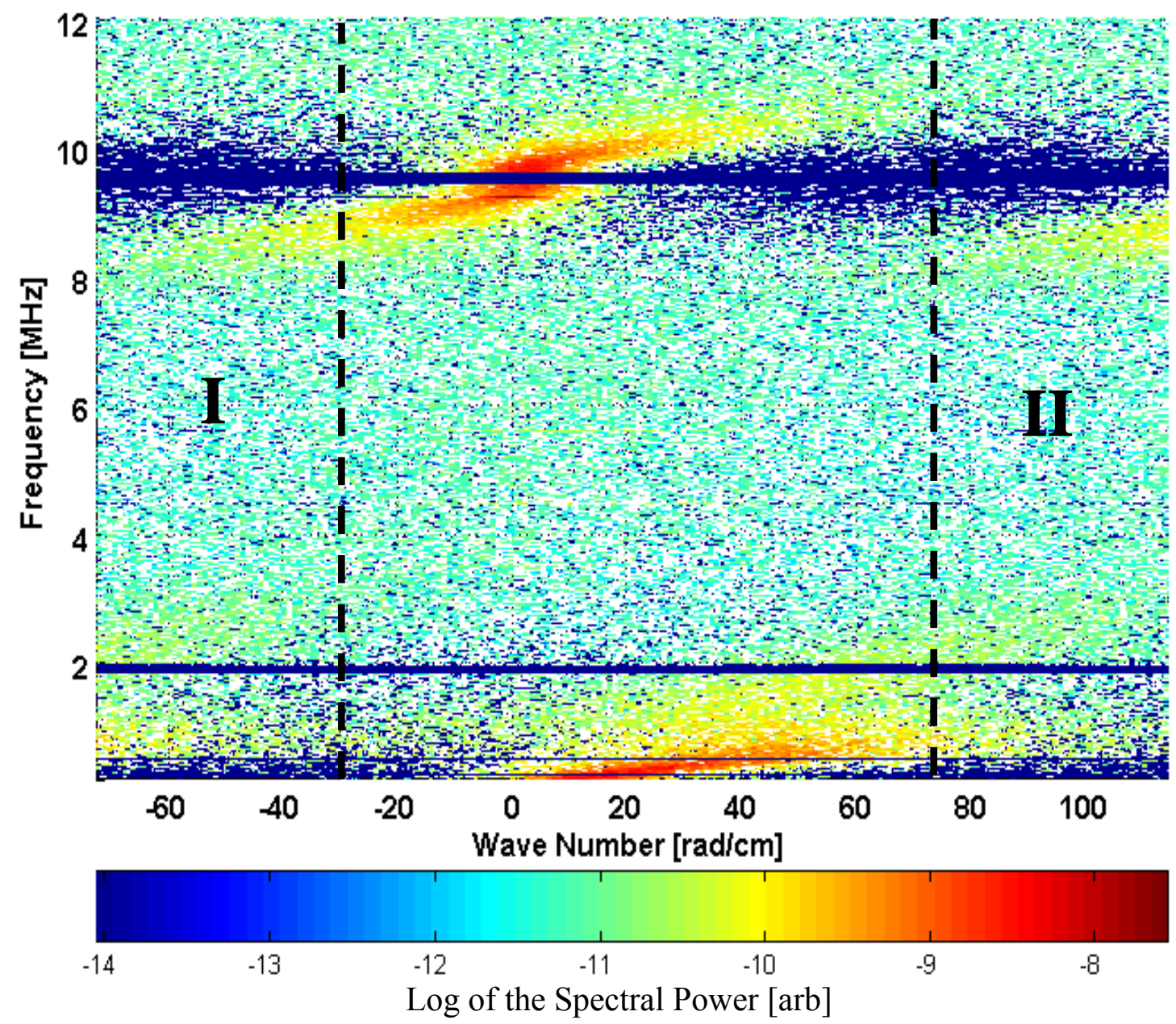

Figure 7.11 Spectral density from the electrostatic double probe oriented $45^{\circ}$ to the magnetic field at $r=$ $4.5 \mathrm{~cm}$ for a magnetic field of $800 \mathrm{G}$, neutral pressure of $8 \mathrm{mTorr}$, and rf driving frequency of $9.5 \mathrm{MHz}$. The vertical dashed lines indicate where region I $(k \leq-30 \mathrm{rad} / \mathrm{cm})$ is phase wrapped to region II. A suppression window at $9.5 \pm 0.1 \mathrm{MHz}$ is applied to enhance the fluctuations.

An example of an electrostatic probe spectral density measurement for the probe tips rotated to $45^{\circ}$ and after phase unwrapping is shown in Figure 7.11. The spectral density region $(-72.8<k<-30 \mathrm{rad} / \mathrm{cm})$ to the left of the dashed line (I) is unwrapped to region II, increasing the effective wave number range to $k \sim 115.6 \mathrm{rad} / \mathrm{cm}$. The increased probe wave number range permits a rough estimate of the expected frequencies of waves with wave numbers that can be observed with the CTS system - for these plasma conditions. As previously noted, the largest scattered powers were obtained with the 
calibration target mirror set to $90^{\circ}$. Thus, fluctuations with $k_{\perp} \sim 89 \mathrm{rad} / \mathrm{cm}$ are of particular interest. Based on Figure 7.11, fluctuations at $k_{\perp} \sim 89 \mathrm{rad} / \mathrm{cm}$ are most likely to appear in the CTS measurements for $r=4.5 \mathrm{~cm}$ at frequencies $200 \mathrm{kHz}$ to $3 \mathrm{MHz}$ and from the rf driving frequency $(9.5 \mathrm{MHz})$ to approximately $12.5 \mathrm{MHz}$. Clearly, if the CTS system were capable of detecting fluctuations at perpendicular wave numbers less than 40 $\mathrm{rad} / \mathrm{cm}$, the chances of obtaining a measureable signal would be greatly increased. Note also that the wave number and frequency dependence of the broad spectrum of higher frequency fluctuation (LSB and USB waves) waves is nearly identical to that of the ( $340 \mathrm{kHz}$ ) fluctuations.

The radial location of the maximum low frequency $(\sim 340 \mathrm{kHz})$ fluctuation amplitude for the each of the ten sets of $\mathrm{rf}$ frequencies and magnetic field strengths specified in Figure 7.1 was determined by integrating the spectral density across a window of $55 \mathrm{kHz}$ centered on a frequency of $340 \mathrm{kHz}$ and over the perpendicular wave number range of 10 to $40 \mathrm{rad} / \mathrm{cm}$. The integrated values obtained with the probe tips aligned perpendicular to the equilibrium magnetic field direction are shown in Figure 7.12. For all source configurations, the $\sim 340 \mathrm{kHz}$ fluctuations are clearly localized to the plasma edge, $r \sim 4.5 \mathrm{~cm}$. At the lowest magnetic field strengths investigated (650 $\mathrm{G}$ and $800 \mathrm{G})$ at a rf frequency of $9.5 \mathrm{MHz}$ (Figure 7.12a), the fluctuations extended deeper into the plasma; into about $r=2.5 \mathrm{~cm}$. The $r=4.5 \mathrm{~cm}$ location of the maximum in fluctuation amplitude corresponds to the same radial location at which increased electron temperatures were observed for the same plasma conditions (see Figure 7.4). Interestingly, the previous measurements of perpendicularly propagating electrostatic fluctuations in HELIX found the largest wave amplitudes closer to the axis of the source. ${ }^{9}$ 

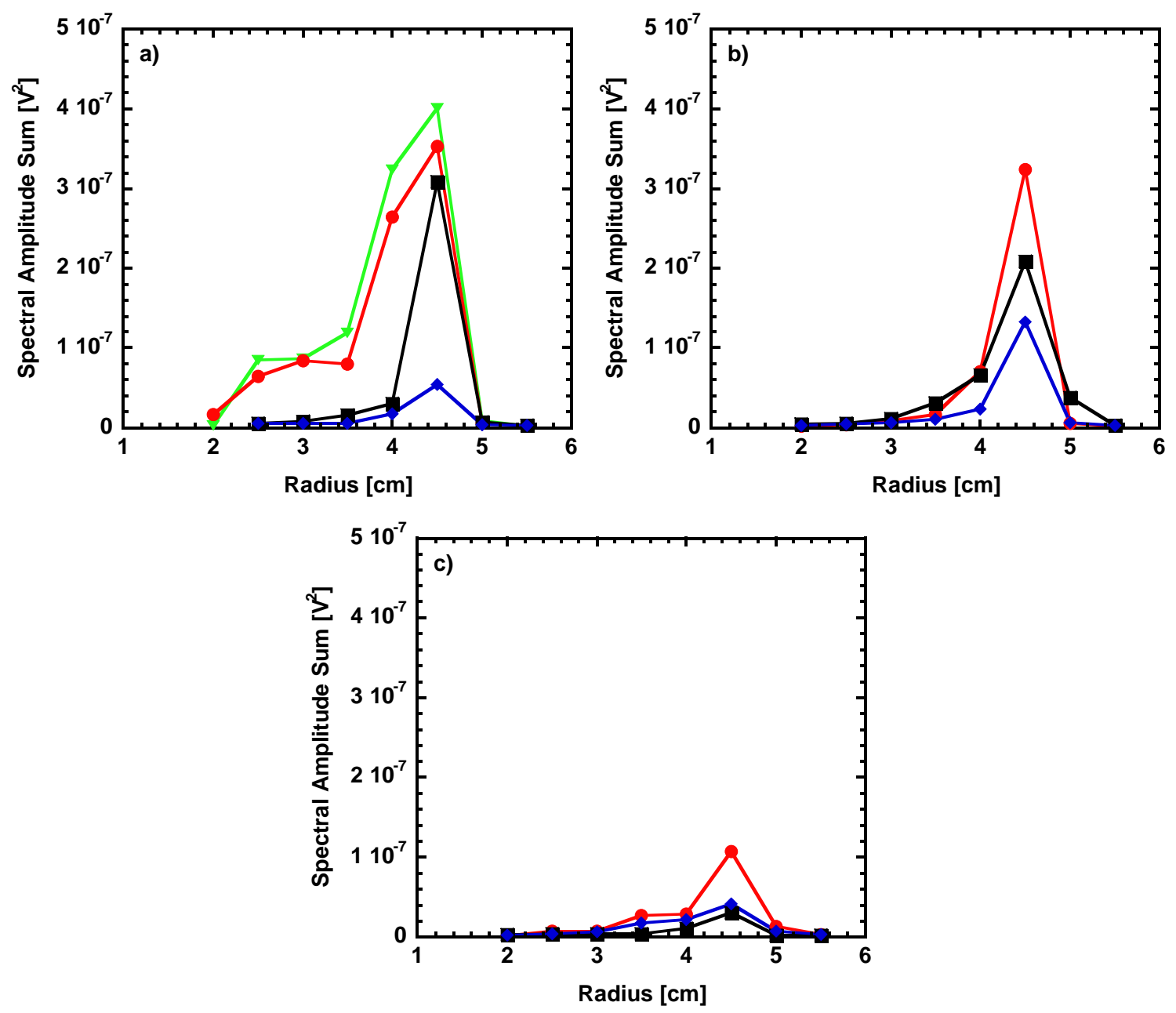

Figure 7.12 Radial profiles of the average spectral density integrated over a $55 \mathrm{kHz}$ window centered at $340 \mathrm{kHz}$ and over the wave number range of 10 to $40 \mathrm{rad} / \mathrm{cm}$ for $\mathrm{rf}$ frequencies of (a) $9.5 \mathrm{MHz}$ (b) 11.5 $\mathrm{MHz}(\mathrm{c}) 13.5 \mathrm{MHz}$ at magnetic fields of $(\nabla) 650 \mathrm{G}(\bullet) 800 \mathrm{G}(\boldsymbol{\nabla}) 950 \mathrm{G}(\diamond) 1100 \mathrm{G}$.

For the plasma conditions with the largest fluctuation amplitudes in Figure 7.12 ( $650 \& 800 \mathrm{G}$ at $r=4.5 \mathrm{~cm}$ ), the spectral density was integrated over $200 \mathrm{kHz}$ to $2 \mathrm{MHz}$ and the resulting wave number spectrum is shown in Figure 7.13. The peak amplitude as a function of perpendicular wave number ranges from $\sim 20$ to $35 \mathrm{rad} / \mathrm{cm}$ and by $90 \mathrm{rad} / \mathrm{cm}$ the fluctuation amplitude is indistinguishable from the noise level. The spectral amplitudes between $\sim 35$ and $50 \mathrm{rad} / \mathrm{cm}$ decay as $\sim k^{-4.5}$ indicating a turbulent cascade at a much faster rate than the Kolmogorov energy transfer rate for an inertial range $\left(k^{-5 / 3}\right)$. Such a large spectral decay rate implies that the waves are being actively dissipated or 
damped over the measured wave number range. An important note about the spectral decay exponent is that it depends upon the radial location and other plasma parameters (magnetic field, rf frequency, etc.), thus the decay rate is not universal. This means that the turbulence is inhomogeneous and indicates that the energy influx at larger scales is strongly dependant on the excitation mechanism coupling energy into the fluctuations. Similar inhomogeneous turbulence was reported in another helicon plasma source and was believed to arise from nonlinear drift wave interactions in a region of radially sheared azimuthal flows. ${ }^{11}$

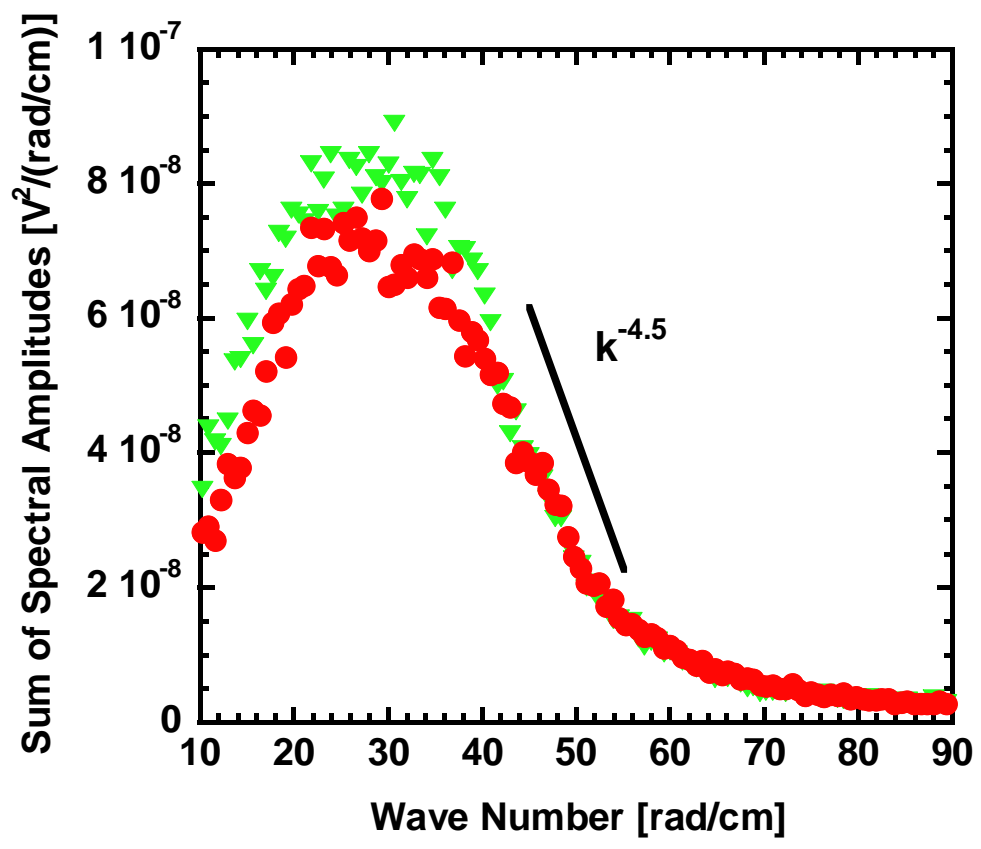

Figure 7.13 Spectral density amplitude integrated from $200 \mathrm{kHz}$ to $2 \mathrm{MHz}$ for magnetic fields of $(\nabla) 650$ $\mathrm{G}$ and $(\bullet) 800 \mathrm{G}$ and an rf frequency of $9.5 \mathrm{MHz}$.

Assuming the potential fluctuations are Boltzmann-like, $\left(\tilde{n} / n_{0}\right) \sim\left(e \tilde{\phi} / k_{B} T_{e}\right)$, the magnitude of the corresponding density fluctuations can be estimated from the measured electrostatic fluctuation amplitudes. For an electron temperature of $3.5 \mathrm{eV}$, the relative fluctuations in density are shown in Figure 7.14 for the same conditions as Figure 7.13. 
Comparison of the relative density fluctuations to the CTS system sensitivities listed in Table 6.4 suggests that for scattering from the fluctuations with perpendicular wave numbers ranging from 60 to $90 \mathrm{rad} / \mathrm{cm}$, the scattered power will be on the order of $\sim 10^{-12}$ $\mathrm{W}$ for a plasma density of $10^{13} \mathrm{~cm}^{-3}$. However, the measured density at $r=4.5 \mathrm{~cm}$ is only $\sim 10^{12} \mathrm{~cm}^{-3}$ and therefore the expected scattered power is an order of magnitude smaller, $10^{-14} \mathrm{~W}$. A scattered power of $10^{-14} \mathrm{~W}$ is just below the current calculated sensitivity of the CTS system. Nonetheless, the plasma conditions with the largest observed fluctuations ( $\mathrm{rf}$ frequency of $9.5 \mathrm{MHz}$ and a magnetic field of $800 \mathrm{G}$ ) were again examined with the CTS system after the detector-mixer was repaired and re-installed.

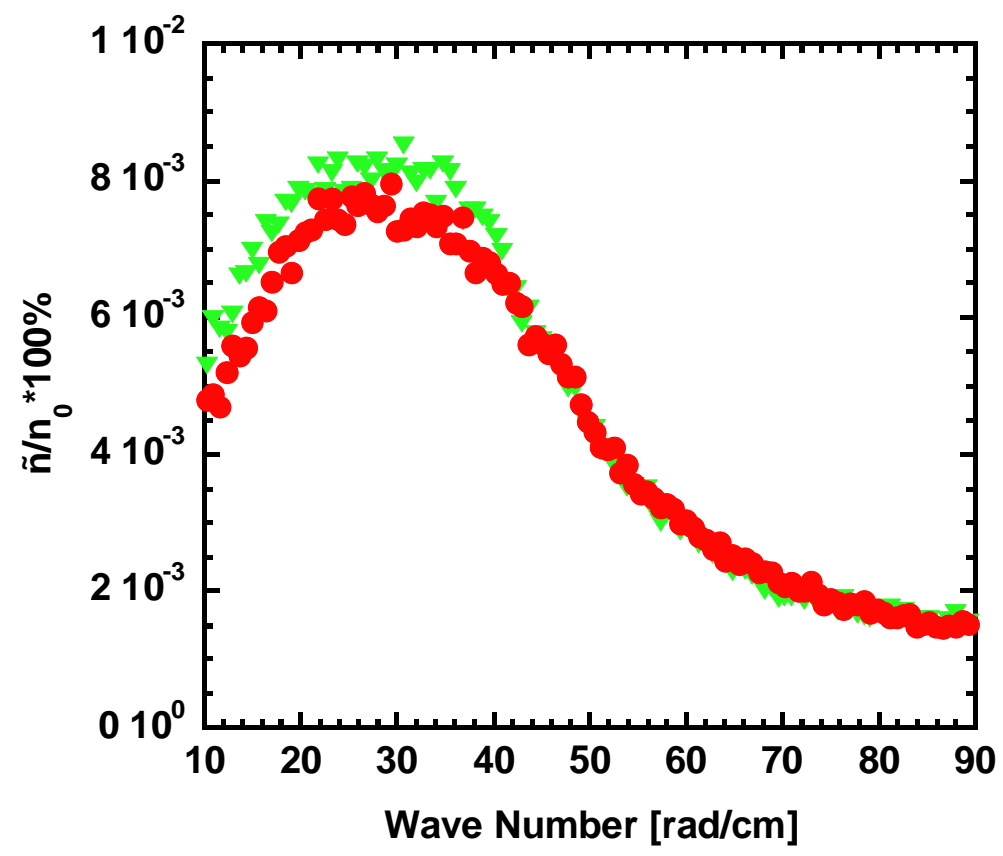

Figure 7.14 $\tilde{n} / n_{0}$ based on the measured electrostatic fluctuation amplitudes integrated over $200 \mathrm{kHz}$ to 2 $\mathrm{MHz}$ at magnetic fields of $(\nabla) 650 \mathrm{G}$ and $(\bullet) 800 \mathrm{G}$ for an rf frequency of $9.5 \mathrm{MHz}$. 


\subsection{Collective Thomson Scattering Measurements - Part 2}

Because there was some indication that the $\sim 2.5 \mathrm{MHz}$ noise signal vanished when the $300 \mathrm{GHz}$ source was unbiased, the effects of varying the DC bias of the source on the source output power and noise characteristics were investigated. To eliminate the possibility of damaging the mixer-detector, a standard optical chopper was placed directly in front of the $300 \mathrm{GHz}$ source to provide an IF signal in the mixer-detector while the signal through the reference leg was measured with a spectrum analyzer. The optical chopper was needed to generate an IF signal in the mixer-detector without modulating the bias voltage (which was the standard method creating a system calibration IF signal). For DC bias voltages from 0 (ground) to 8 Volts, the peak signal at the chopping frequency was normalized to the largest peak signal and plotted in Figure 7.15. For comparison, the original measurements using the rotating grating shown in Figure 6.23 and the output measurements provided by the manufacturer are also plotted in Figure 7.15. While the maximum power still occurred at a bias voltage of $6 \mathrm{~V}$, the sharp decrease in our measured power values at a bias voltage of $8 \mathrm{~V}$ was unexpected. Attempts to resolve the discrepancies in power output versus bias voltage and to explain the apparent broadband frequency noise through discussions with Radiometer Physics and engineers at Virginia Diodes Inc., a company producing similar products as Radiometer Physics, were again unsuccessful. Deferring to the manufacturer's calibration data, for the remainder of this work the bias voltage was set to $\sim 7.75 \mathrm{~V}$ for active measurements and $0 \mathrm{~V}$ when measuring noise levels. 


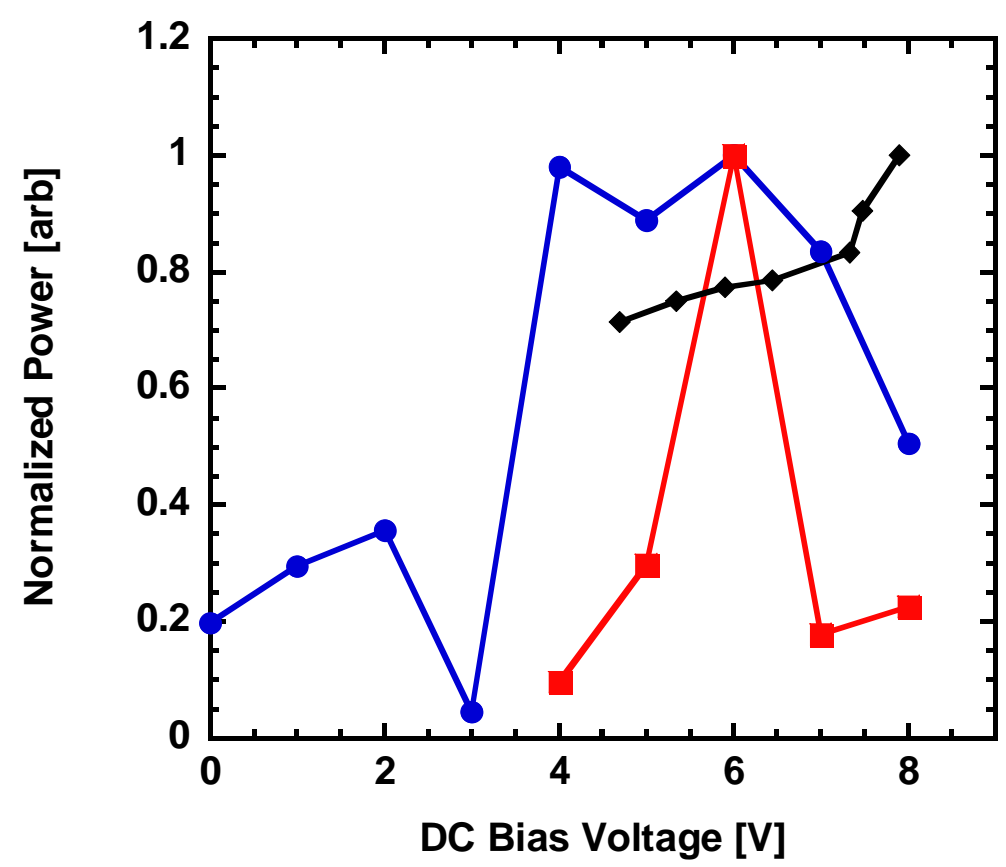

Figure 7.15 Revised normalized signal amplitude $(\bullet)$, original signal amplitude $(\square)$ and the normalized $300 \mathrm{GHz}$ source output powers $(\diamond)$ from Table 6.1 .

For comparison with measurements during normal, "plasma," operation, the output of the mixer-detector was recorded at a digitization rate of $100 \mathrm{MHz}$ and averaged over twenty measurements with the $300 \mathrm{GHz}$ Varactor DC bias voltage grounded and with the bias voltage at $7.75 \mathrm{~V}$ (for two separate measurements). The results are shown in Figure 7.16. The broad $2.5 \mathrm{MHz}$ noise signal is absent when the source is grounded and while the source is biased at $7.75 \mathrm{~V}$, the noise amplitude varies significantly. For all other bias voltages, the same effect (noise signal amplitude fluctuating in time) occurred. So although the $\sim 2.5 \mathrm{MHz}$ noise vanishes when the Varactor DC bias is set to ground, the manufacturer's output power measurements also indicate that the output power of the source is substantially reduced at zero bias. Therefore, measurements were made at Varactor DC bias voltages of $\sim 7.75 \mathrm{~V}$ and $0 \mathrm{~V}$. 


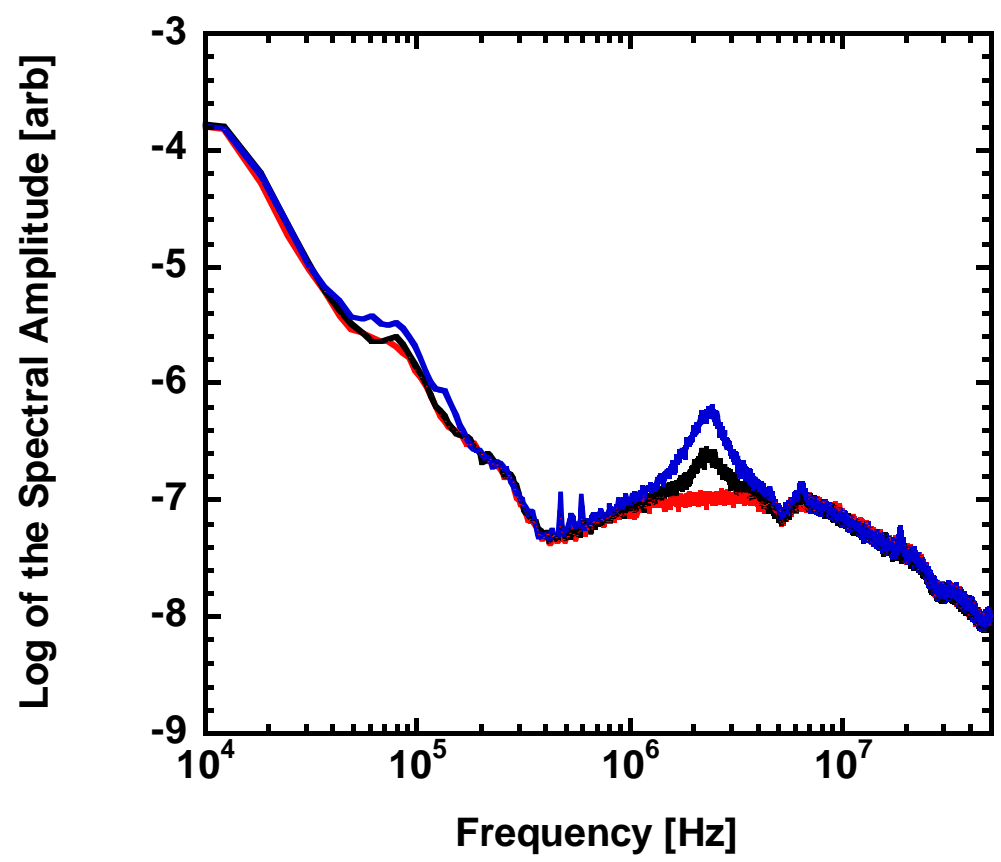

Figure 7.16 Detector-mixer power spectra in the absence of plasma with the DC bias voltage ( grounded, (-) $8 \mathrm{~V}$ measurement\#1, and (L) $8 \mathrm{~V}$ measurement \#2.

Shown in Figure 7.17 are CTS system scattering measurements for an $\mathrm{rf}$ frequency of $9.5 \mathrm{MHz}$, a magnetic field strength of $800 \mathrm{G}$, and a neutral pressure of 8 mTorr. These parameters correspond to the conditions with the largest fluctuation amplitudes as measured with the electrostatic double probe. To maximize the signal-tonoise, each measurement was accomplished by acquiring 100 time series of 262,144 points at a digitization rate of $100 \mathrm{MHz}$. The time series were then subdivided into 16 individual time series subsets before applying a Blackman window and then averaging the FFTs. By recording 100 individual time series and subdividing each time series into 16 subsets, the errors arising from normally distributed random noise was reduced by a factor of $\sim 1 / \sqrt{1600}$. With the vacuum mirror set to collect at $90^{\circ}$, measurements at the $r$ $=4.5 \mathrm{~cm}$ location with the entire system operating and with the collection optics window 
blocked were obtained to identify any scattered signals in the power spectrum of the CTS measurements.
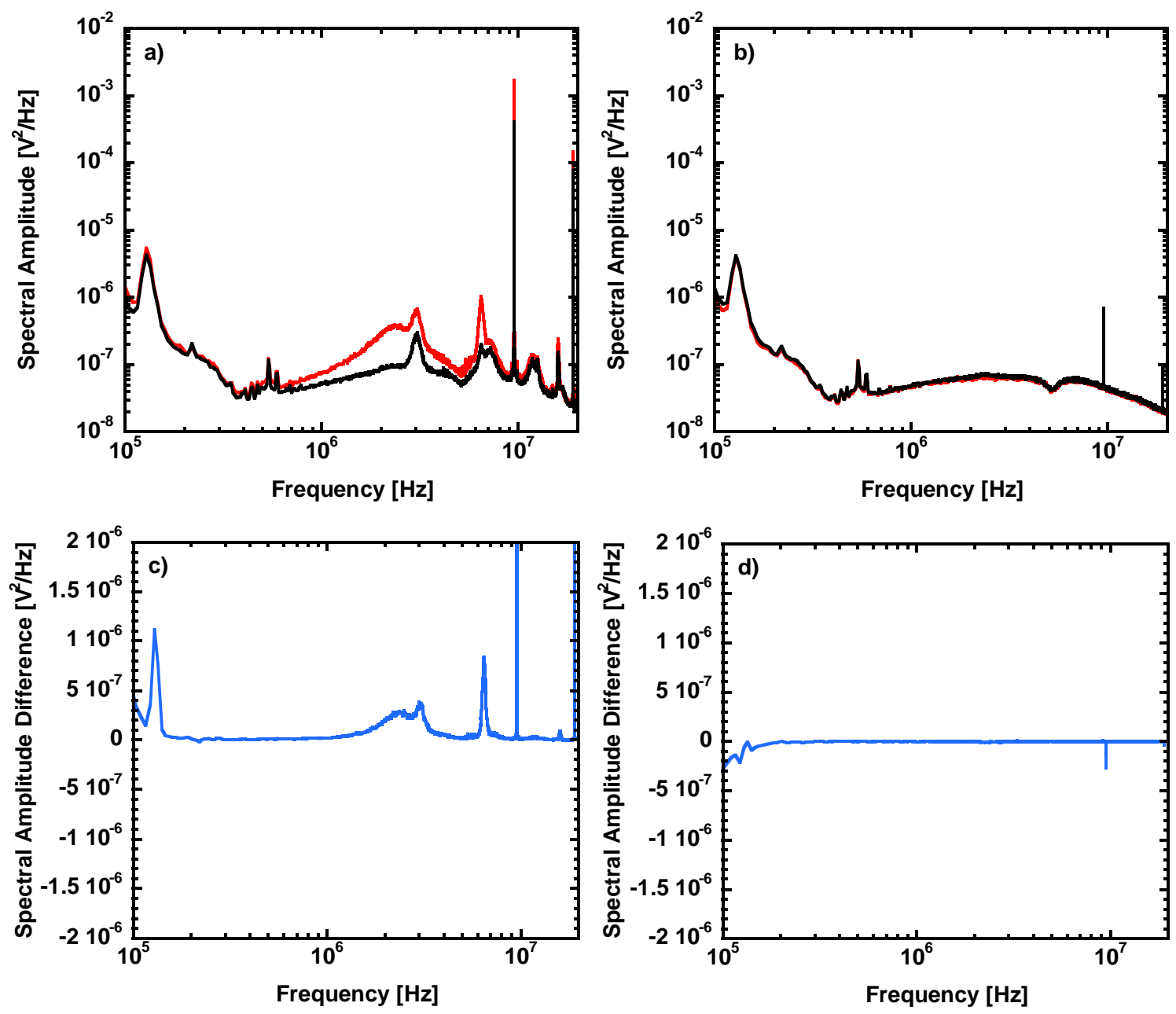

Figure 7.17 Measured power spectra for the full CTS system (_) and the with the interaction beam blocked at the collection optics (_) with DC bias voltages of (a) $7.75 \mathrm{~V}$ and (b) $0 \mathrm{~V}$ for an rf frequency of $9.5 \mathrm{MHz}$, a magnetic field strength of $800 \mathrm{G}$, a neutral pressure of $8 \mathrm{mTorr}$, and at $r=4.5 \mathrm{~cm}$. The differences between the full and blocked spectral measurements are shown in (c) and (d) for the corresponding DC bias voltages.

As expected, for a DC bias voltage of $7.75 \mathrm{~V}$ (Figure 7.17a) the broadband noise appears at $\sim 2.5 \mathrm{MHz}$. Also evident in Figure $7.17 \mathrm{a}$ are peaks at beat frequencies between the noise and the fundamental and harmonics of the rf driving frequency at $9.5 \mathrm{MHz}$. Because of the beating of the noise with the rf frequency, it is difficult to distinguish any scattered signals at frequencies just above or below the rf frequency. Close examination 
of the mixer-detector signal at the rf frequency indicates a significant difference in the measured amplitude between the full system measurement and when the collection optics were blocked (Figure 7.17c). Although initially suggestive of real scattered power at the rf frequency, and therefore possible scattering from slow waves, an analysis of similar measurements at multiple radial locations found that the difference between the full system and blocked collection found no correlation of the amplitude difference with radius. In fact, for adjacent radial locations, the difference often changed from positive to negative. The most likely explanation is that the $\sim 2.5 \mathrm{MHz}$ noise level affects the overall signal amplitude at $\mathrm{MHz}$ frequencies (this can be seen in Figure 7.17) and since the noise level varies over the period of minutes, the mixer signal amplitudes at the rf frequency vary randomly as well.

Also as expected, the broadband noise at $\sim 2.5 \mathrm{MHz}$ vanishes for measurements with the Varactor DC bias voltage grounded (Figure 7.17b). Comparison with measurements at the same conditions but the collection optics blocked indicates no significant peaks in the power spectrum other than at the fundamental $\mathrm{rf}$ frequency (Figure $7.17 \mathrm{~d}$ ). The amplitude difference at the $\mathrm{rf}$ frequency is negative and therefore consistent with no scattering of the $300 \mathrm{GHz}$ microwave signal by fluctuations at the rf frequency. 


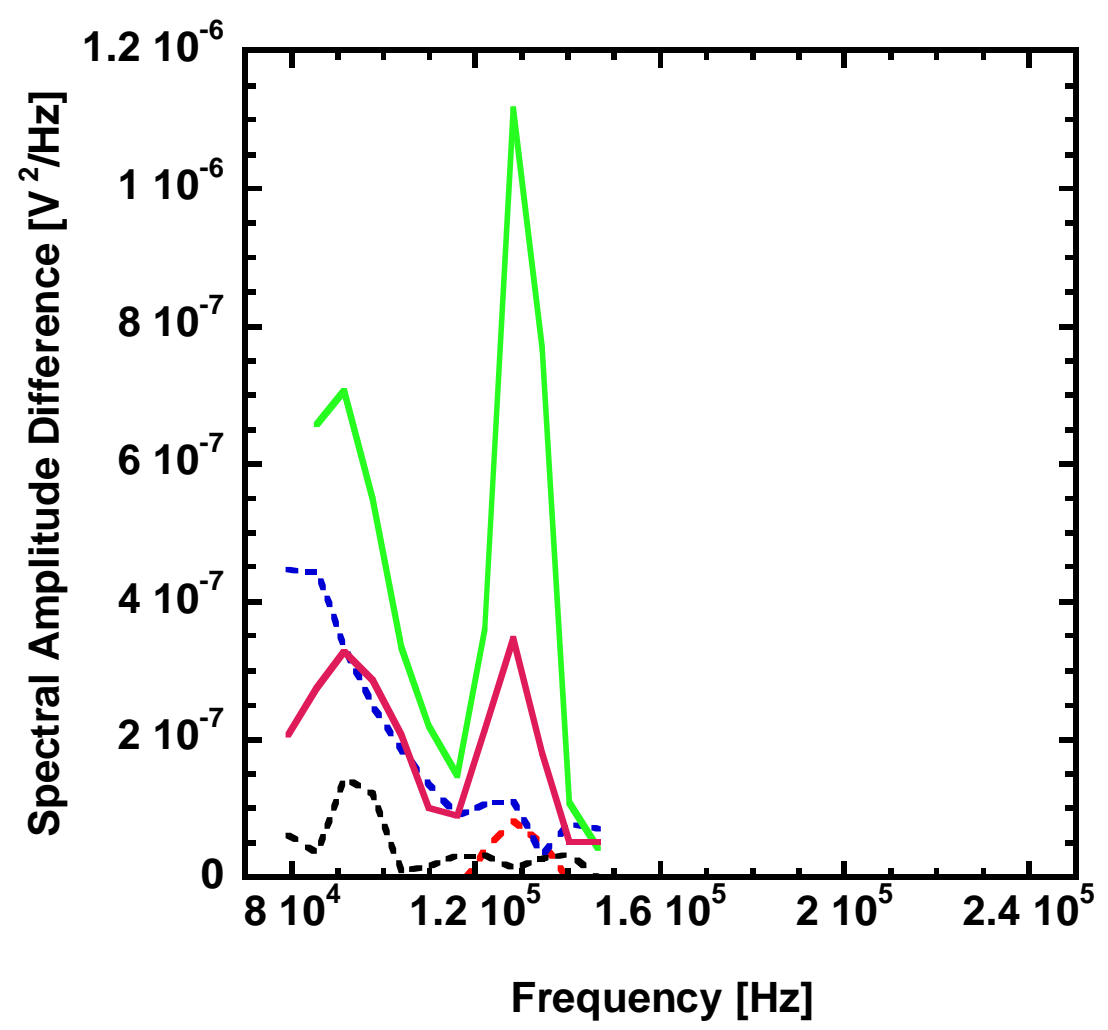

Figure 7.18 Radial measured power spectral differences between the full CTS system and the interaction beam blocked at the collection optics with DC bias voltage of $7.75 \mathrm{~V}$ for an rf frequency of $9.5 \mathrm{MHz}$, a magnetic field strength of $800 \mathrm{G}$, a neutral pressure of 8 mTorr, at radii of $r=3.0 \mathrm{~cm}(n \mathrm{rr}), r=3.5 \mathrm{~cm}$

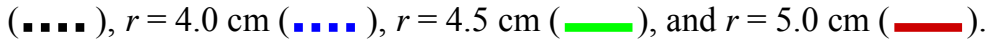

However, at frequencies on the order of $125 \mathrm{kHz}$ (much lower than the broadband noise peak), there is a significant difference in mixer-detector amplitudes between the full system and blocked collection measurements. Shown in Figure 7.18 are the mixerdetector power spectra obtained at five different radial locations for the same parameters as Figure 7.13. The maximum amplitude difference in the frequency band $80 \mathrm{kHz}-150$ $\mathrm{kHz}$ is observed at $r=4.5 \mathrm{~cm}$. For radii larger and smaller than $4.5 \mathrm{~cm}$, the scattered power in this band of frequencies decreases. The difference in spectral power summed over the frequency range $80-150 \mathrm{kHz}$ is shown in Figure 7.19 as a function of radius. Also shown in Figure 7.19 are the electrostatic probe amplitudes for perpendicular wave numbers from 10 to $40 \mathrm{rad} / \mathrm{cm}$ integrated over a $55 \mathrm{kHz}$ band centered at $340 \mathrm{kHz}$ for the 
same plasma parameters and at the same radial locations (from Figure 7.12a). The similarity in radial profiles is striking and provides the first evidence that the CTS system successfully detected electrostatic fluctuations with wave numbers $\sim 90 \mathrm{rad} / \mathrm{cm}$ at frequencies $\sim 125 \mathrm{kHz}$.

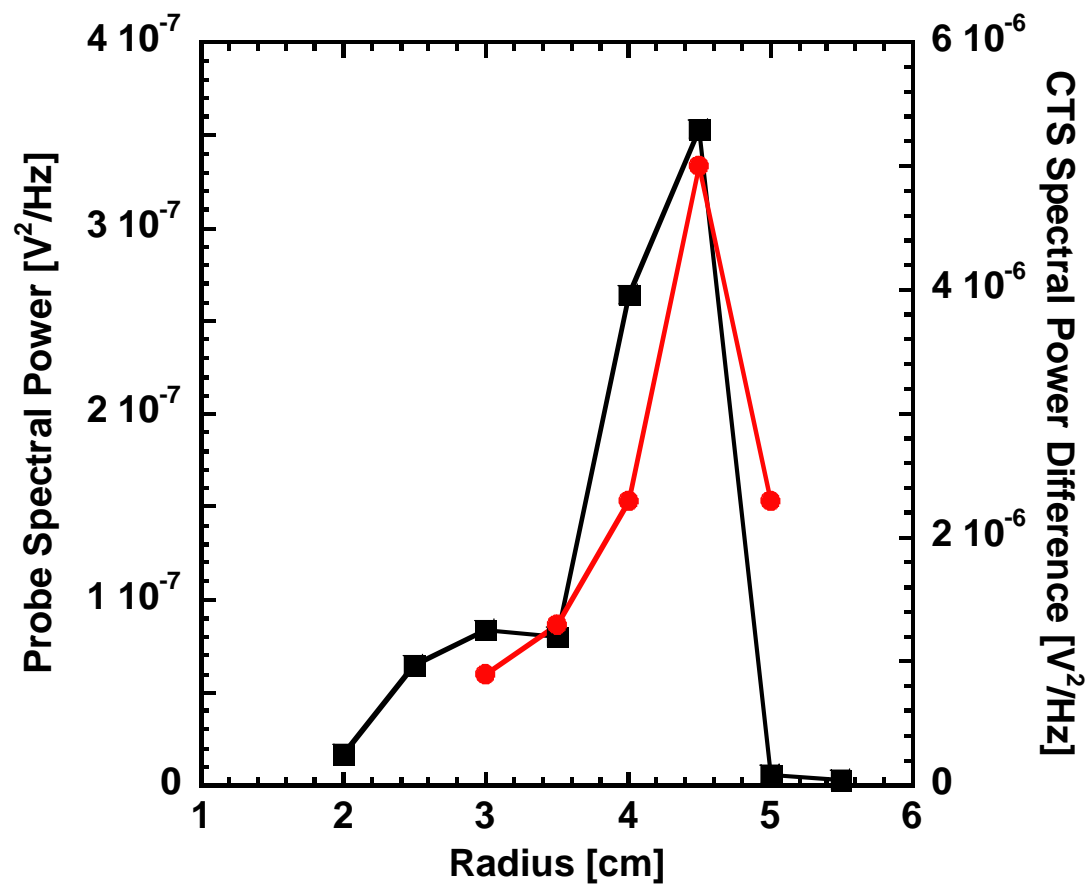

Figure 7.19 Radial profiles of the integrated spectral power for the electrostatic double probe ( $\square$ ) and the CTS diagnostic (O) for an rf frequency of $9.5 \mathrm{MHz}$, a magnetic field strength of $800 \mathrm{G}$, and a neutral pressure of 8 mTorr. The electrostatic spectral power is integrated for a range of $55 \mathrm{kHz}$ centered at 340 $\mathrm{kHz}$ and the CTS spectral power is integrated over the range between $80-150 \mathrm{kHz}$.

These first CTS system measurements are clearly at the very limits of sensitivity and signal-to-noise of the CTS diagnostic. If a means to keep the broadband frequency noise constant in time could be found, it is possible that the existing CTS system could successfully measure scattered power at higher frequencies and over a wider range of perpendicular wave numbers. However, the limitations of the current CTS system prevent its use for detailed studies of the large perpendicular wave number fluctuations in 
HELIX. Therefore, the electrostatic probe was re-introduced into the experiment to more thoroughly investigate the nature of the observed fluctuations.

\subsection{Electrostatic Wave Investigations}

As noted previously, the similarity of the dispersion patterns of the low frequency (LF), low side band (LSB), and upper side band (USB) fluctuations presented in Figure 7.9 and Figure 7.10 suggest that these fluctuations are parametrically driven waves coupled to the rf pump wave. Parametrically driven waves generally considered to be ionacoustic and lower hybrid or TG waves have been reported before in a number of helicon source experiments ${ }^{9,10,12}$ and have been suggested as a primary source of electron scattering, and therefore energy coupling in helicon sources. ${ }^{13}$ Corr et al. have reported spatially localized ion acoustic waves in low pressure helicon sources believed to be correlated with the gradient of the radial plasma pressure, ${ }^{14}$ while ion cyclotron waves have also been observed from parametric interactions with the rf pump wave. ${ }^{15}$

The standard criteria for establishing the three wave parametric coupling is through the conservation of energy $\left(f_{0}=f_{1} \pm f_{2}\right)$ and conservation of momentum $\left(k_{0}=k_{1} \pm k_{2}\right)$. For our purposes we define $f_{0} \equiv f_{R F}$ and $k_{0} \equiv k_{R F}$ to be the rf driving frequency and wave number, respectively. An additional example of the similarity in dispersion patterns of the waves under consideration is shown below in Figure 7.20 for an rf frequency of $9.5 \mathrm{MHz}$ and a magnetic field strength of 650 Gauss at $r=4.5 \mathrm{~cm}$. To suppress the enormous signal at $9.5 \mathrm{MHz}$ and its harmonics, the spectral densities within $\pm 200 \mathrm{kHz}$ of multiples of the rf frequency are not shown. At the rf frequency and its harmonics, the measurements indicate that $k_{R F} \approx 0$. Thus, $0 \approx k_{1} \pm k_{2}$ is required to 
satisfy the wave number matching condition for waves parametrically beating with the rf pump wave or its harmonics.

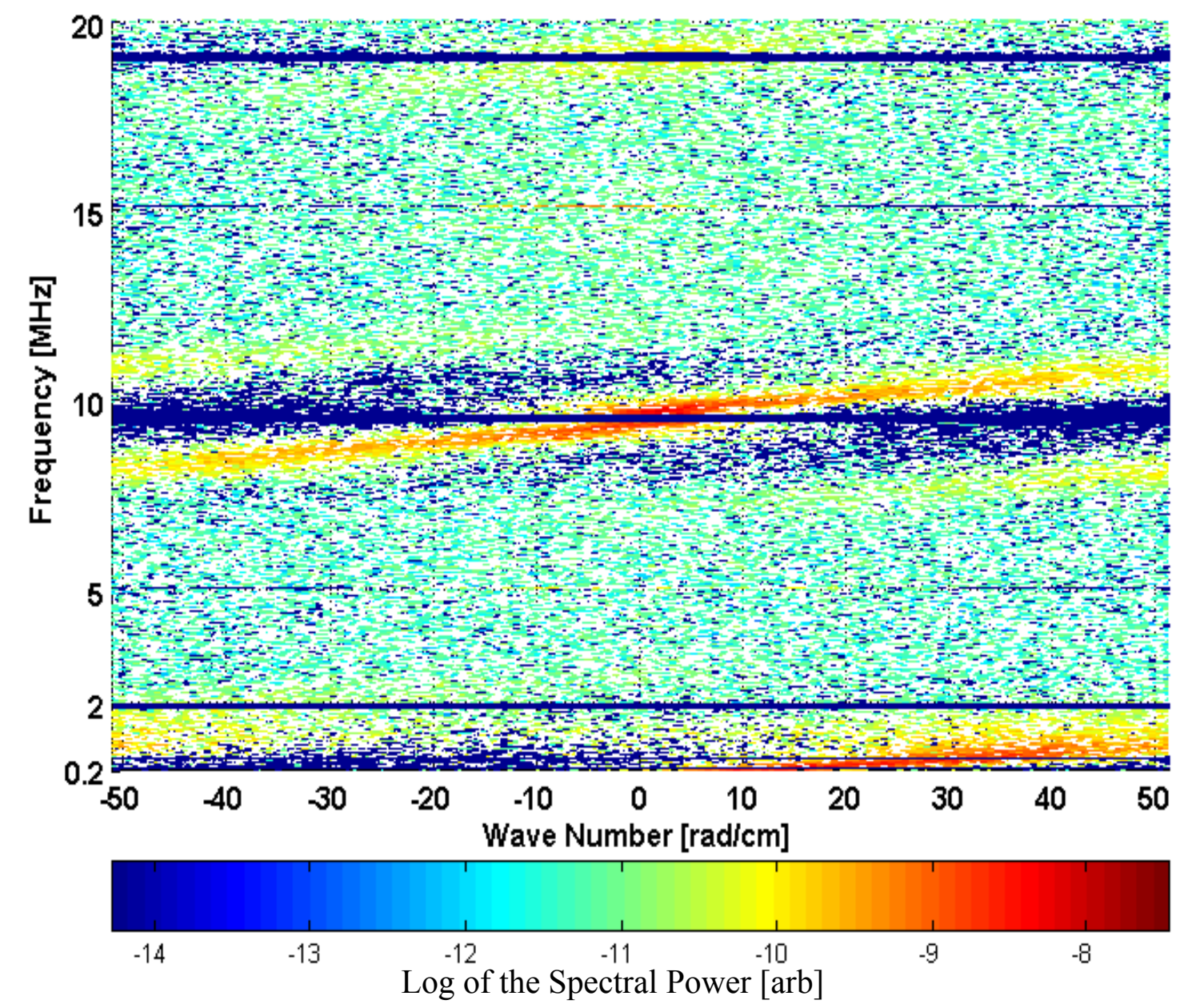

Figure 7.20 Spectral density obtained with the electrostatic double probe perpendicular to the magnetic field at $r=4.5 \mathrm{~cm}$ for a magnetic field strength of $650 \mathrm{G}$, neutral pressure of $8 \mathrm{mTorr}$, and an rf driving frequency of 9.5 MHz. The spectral density within $9.5 \pm 0.2 \mathrm{MHz}$ is suppressed to accentuate the turbulent fluctuations.

Slices through the spectral density along the wave number axis at frequencies of $500 \mathrm{kHz}, \pm 500 \mathrm{kHz}$ around the rf frequency $(9.5 \mathrm{MHz})$, and $\pm 500 \mathrm{kHz}$ around the first harmonic of the rf frequency (19 MHz) are shown in Figure 7.21. The spectral density was integrated over a band $42 \mathrm{kHz}$ wide for each frequency shown. 


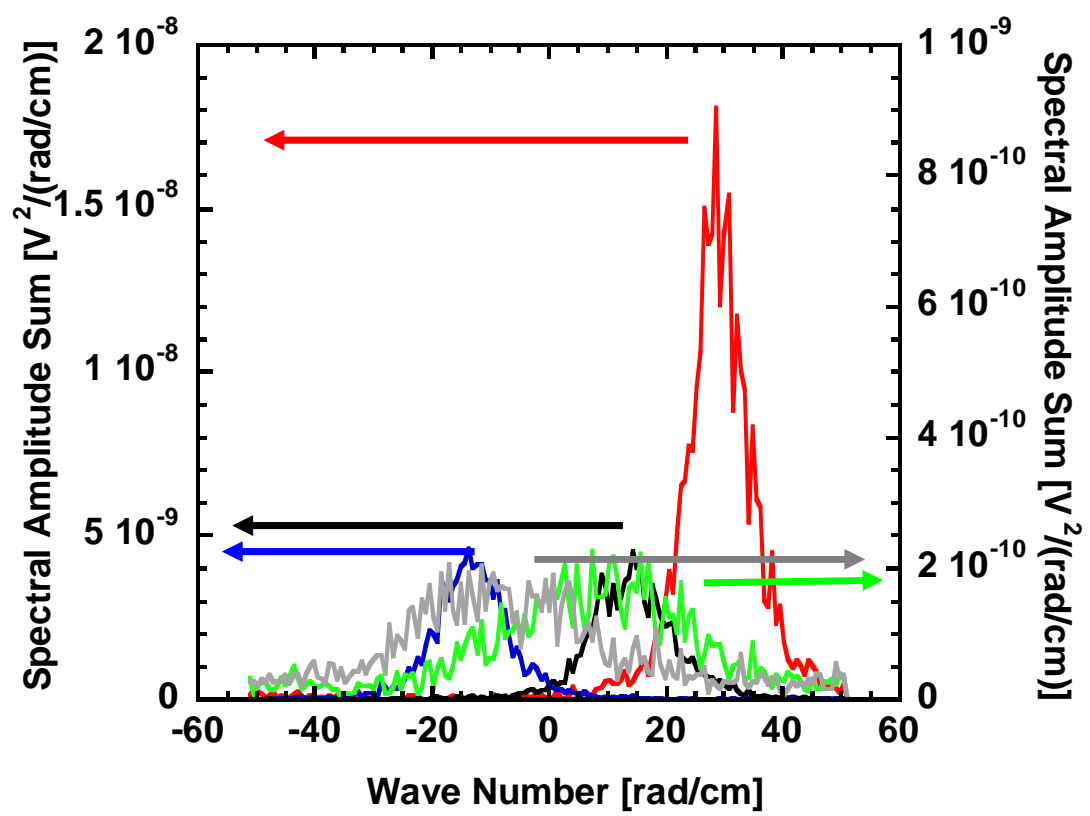

Figure 7.21 Slices through the spectral density from Figure 7.21 at (_) $500 \mathrm{kHz}$, (ـ) $9 \mathrm{MHz}$, (_) $10 \mathrm{MHz},(-) 18.5 \mathrm{MHz}$, and (_) $19.5 \mathrm{MHz}$.

For the momentum conservation to be satisfied in a three-wave parametric decay process, the difference in wave number between the waves at $500 \mathrm{kHz}(\mathrm{LF})$ and $10 \mathrm{MHz}$ (USB) should equal the wave number at $9.5 \mathrm{MHz}\left(k_{R F} \approx 0\right)$. Gaussian fits to the LF and USB waves yield wave numbers of $k_{L F}=28.9 \pm 0.7$ and $k_{U S B}=13.9 \pm 0.7 \mathrm{rad} / \mathrm{cm}$, respectively. The calculated wave number difference of $\sim 15 \mathrm{rad} / \mathrm{cm}$ is clearly nonzero. Therefore, although these three waves satisfy the frequency matching condition, their interaction does not conserve momentum.

Further examination of Figure 7.21 reveals that the wave numbers of the USB and LSB $\left(k_{L S B}=-13.3 \pm 0.7\right)$ waves at $10 \mathrm{MHz}$ and $9 \mathrm{MHz}$ sum to zero within the experimental error. Similarly, the wave numbers of the lower and upper side bands around the first harmonic of the rf pump wave, also shown in Figure 7.21, sum to zero. Based solely on the wave number measurements, it would appear that the lower 
frequency band of electrostatic waves arises from the beating together of the LSB and USB waves. Although the sum of the wave number of the LSB and USB waves \pm 500 $\mathrm{kHz}$ around the rf frequency does equal the wave number of the LF wave at $500 \mathrm{kHz}$, none of the three waves are actively driven. If we require interaction with the rf pump wave or its harmonics, the total wave number matching condition can still be satisfied through a four-wave interaction $\left(k_{U S B}-k_{L S B}+k_{R F}=27.2 \pm 1.4 \approx k_{L F}\right)$, but the frequency matching requirement is still violated.

Other observations, reported in Ref. [14] and [15], of parametric instabilities in helicon sources were limited entirely to frequency measurements and the momentum conservation was assumed. The observations reported in Ref. [10] have frequency and wave number measurements roughly consistent with parametric decay, but their entire analysis was based on the measured frequency and wave number spectra "following" the same slope defined by the LF wave (see Figure 1.10). No explicit analysis of the spectral densities that confirmed the parametric conditions were satisfied was reported. Mismatches in the wave number criteria were reported in the parametric decay studies in Ref. [9], but the limited measureable wave number range prevented a more thourough examination. Therefore, these measurements, while superficially similar to expectations for a three-wave parametric decay process, are not consistent with parametric excitation of the low frequency, acoustic-like, waves. The waves are clearly coupled to the USB and LSB waves, but in a complex relationship that is not yet understood. 


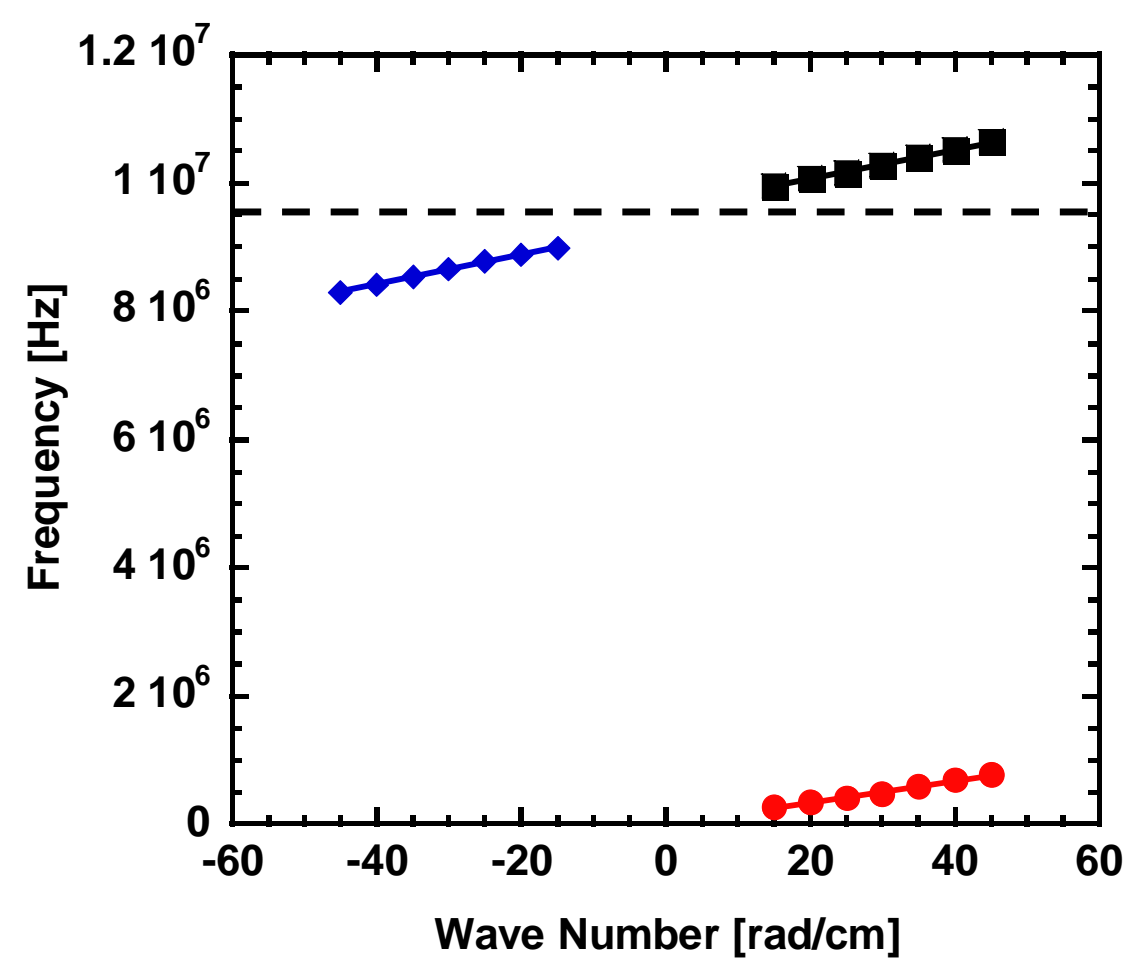

Figure 7.22 Dispersion curves obtained from a series of slices through the spectral densities shown in Figure 7.22, for $(\bullet)$ the LF peaks, ( $\square)$ the USB peaks, and $(\nabla)$ the LSB peaks. The dashed line is the rf frequency $(9.5 \mathrm{MHz})$.

With additional slices through the spectral density plot of Figure 7.20 , the frequency and wave number for the maximum in the spectral density along each slice was determined and the analysis results are shown in Figure 7.22. Note that the phase velocity $\left(\omega / k_{\perp}\right)$ of the LSB is negative while the group velocity $\left(d \omega / d k_{\perp}\right)$ is positive, i.e. backwards propagation. Backward propagation is characteristic of electrostatic lower hybrid waves or TG waves. ${ }^{9,10,16,17}$ Shown in Figure 7.23 are the magnitudes of the phase velocities, $V_{p}$, of the USB and LSB waves. Langmuir probe measurements at the same source parameters and radial location (see Figure 7.4) yielded an electron temperature of $3 \mathrm{eV}$. The corresponding electron thermal velocity $\left(V_{\text {the }}\right)$ is on the order $7 \times 10^{7} \mathrm{~cm} / \mathrm{s}$, roughly an order of magnitude larger than the measured USB and LSB phase velocity. However, the USB and LSB waves associated with harmonics of the rf pump waves, at 
$36 \mathrm{MHz}$ for example, have phase speeds comparable to the electron thermal speed, $V_{p} \sim$ $0.25 V_{\text {the }}$. At such wave phase speeds, significant Landau damping of the side bands, and therefore electron heating, may occur.

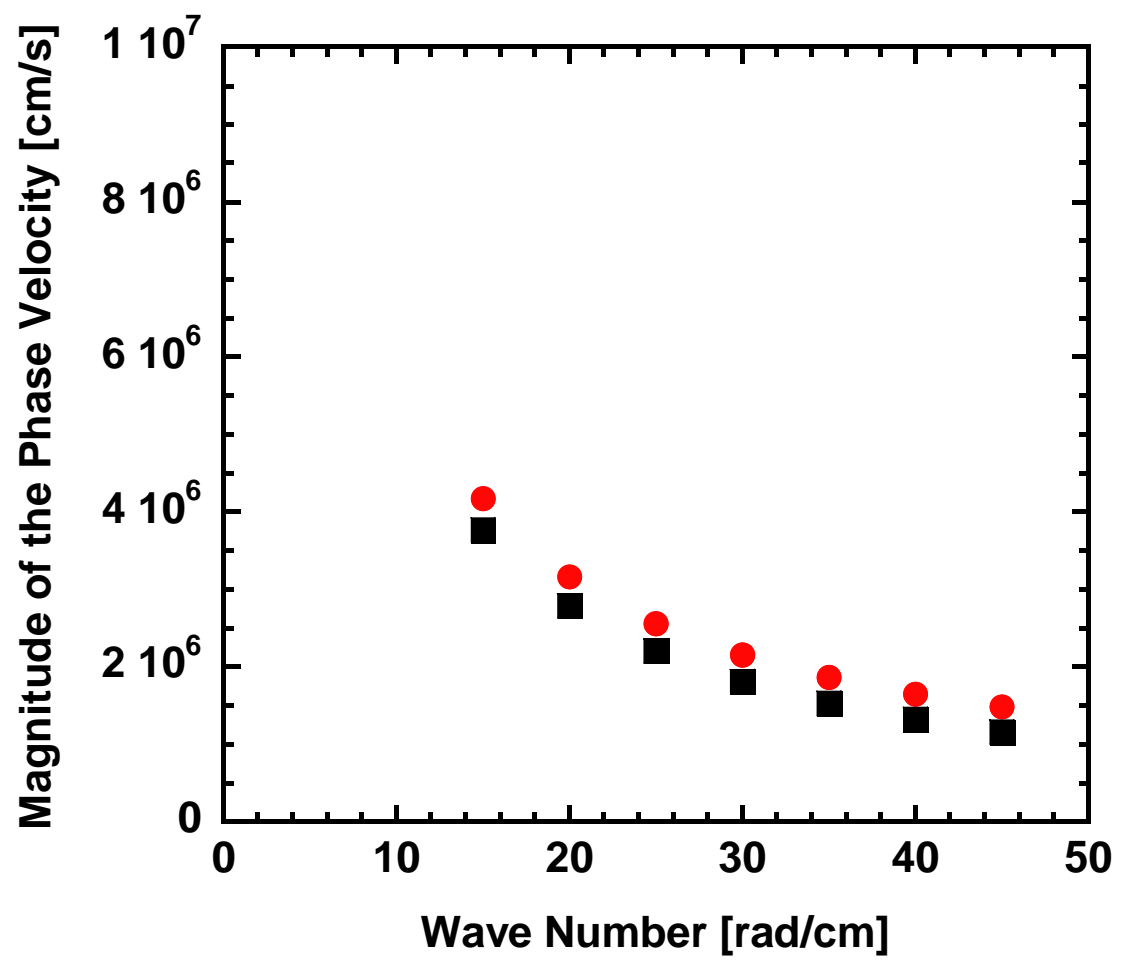

Figure 7.23 Measured phase velocities of the USB fluctuations (๑) and the LSB fluctuations ( $\square$ ).

The absolute frequency and linear relationship between frequency and wave number of the LF waves suggest that the waves might be ion-acoustic fluctuations. ${ }^{9,10}$ The measured LF wave frequencies along with the predicted frequency for an ionacoustic wave at the same wave number in a plasma with an electron temperature of $3 \mathrm{eV}$ are shown in Figure 7.24. The error bars for the predicted ion acoustic wave frequencies are based on the error estimates for the spacing of the two tips of the electrostatic probe. The discrepancy between the measured LF wave properties and those of ion-acoustic waves is essentially a matter of electron temperature, i.e., sound speed. Fitting an ion 
acoustic wave dispersion to the measurements yields an electron temperature of only 0.5 $\mathrm{eV}$; quite different from the measured $3 \mathrm{eV}$. The Langmuir probe and electrostatic double probe measurements were performed at different axial locations in HELIX (see Figure 2.2). However, it is unlikely that the electron temperature varies from $0.5 \mathrm{eV}$ to $3 \mathrm{eV}$ over such a short axial distance.

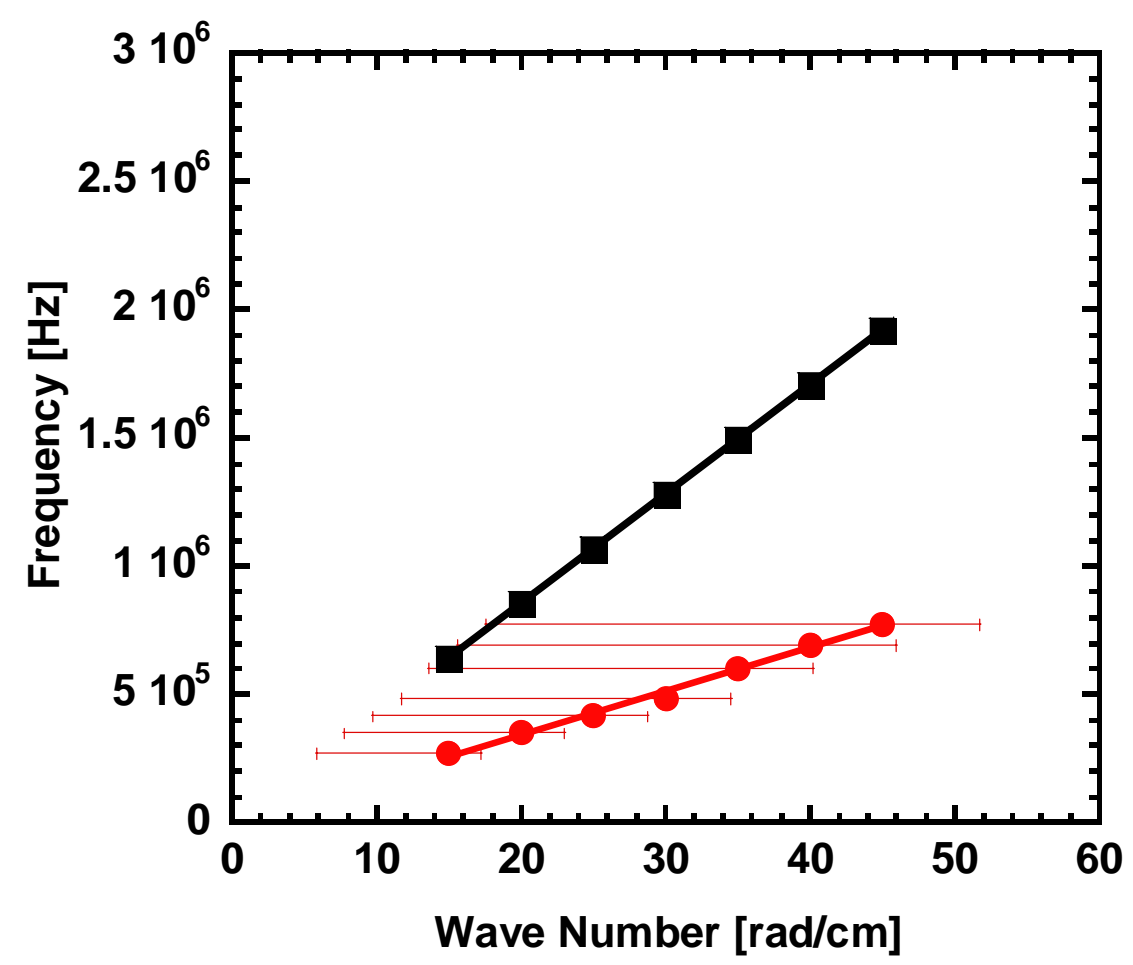

Figure 7.24 Dispersion curves for (๑) the measured LF wave and ( $\square$ ) for an ion-acoustic wave at the same wave number for a plasma with an electron temperature of $3 \mathrm{eV}$.

One of the key issues underlying the electron temperature (sound speed) discrepancy is the effective probe tip separation. The initial assumption was that the center-to-center tip distance accurately describes the probe tip separation. However, the radii of the probe tips are comparable to the center-to-center spacing of the tips. If the outer edges of each tip are included in this analysis, thereby increasing the effective tip separation, the resulting LF dispersion agrees with the expectation for an ion-acoustic 
wave in a plasma with an electron temperature of $3 \mathrm{eV}$ (error bars to the left of the data in Figure 7.24). This dramatic effect of uncertainty in the effective probe tip separation underscores the importance of measuring such short-wavelength fluctuations noninvasively with a CTS-type diagnostic.

The phase velocities of the measured LF waves are on the order of $1.0 \times 10^{5} \mathrm{~cm} / \mathrm{s}$. Assuming a perpendicular ion temperature of $0.2 \mathrm{eV}$, based on LIF measurements made for similar experimental parameters, ${ }^{18}$ the perpendicular ion thermal velocity $\left(V_{t h i}\right)$ is $\sim 7$ x $10^{4} \mathrm{~cm} / \mathrm{s}$. With wave phase velocities on the order of $1.4 V_{t h i}$, it is certainly possible that these driven low frequency waves could Landau damp on, and therefore heat, the ions in the edge of the plasma. The phase velocities of the waves observed by the CTS system, $f$ $\sim 100 \mathrm{kHz}$ and $k_{\perp} \sim 89 \mathrm{~cm} / \mathrm{s}$, are much lower, $V_{p} \sim 0.1 V_{\text {thi }}$, and could therefore also easily couple to the bulk of the ion distribution. 


\section{Chapter 7 References}

${ }^{1}$ J.L. Kline, E.E. Scime, R.F. Boivin, A.M. Keesee, X. Sun, and V.S. Mikhailenko, Phys. Rev. Lett., 88, 195002 (2002).

2 M.J. Burin, G.R. Tynan, G.Y. Antar, N.A. Crocker, and C. Holland, Phys. Plasmas, 12, 052320 (2005).

${ }^{3}$ C. Schroder, O. Grulke, T. Klinger, and V. Naulin, Phys. Plasmas, 12, 042103 (2005).

${ }^{4}$ M. Light and F.F. Chen, Phys. Plasmas, 2, 1084 (1995).

${ }^{5}$ A.L. Peratt, R.L. Watterson, and H. Derfler, Phys. Fluids, 20, 1900 (1977).

${ }^{6}$ W.A. Peebles, N.C. Luhmann Jr., A. Mase, H. Park, and A. Semet, Rev. Sci. Instrum., 52, 360 (1981).

${ }^{7}$ J.L. Kline, Slow Wave Ion Heating and Parametric Instabilities in the HELIX Helicon Source, Ph.D. Dissertation, West Virginia University, Morgantown (2002).

${ }^{8}$ M.E. Koepke and W.E. Amatucci, IEEE Trans. Plasma Sci., 20, 631 (1992).

${ }^{9}$ J.L. Kline and E.E. Scime, Phys. Plasmas, 10, 135 (2003).

${ }^{10}$ M. Kramer, Y.M. Aliev, A.B. Altukhov, A.D. Gurchenko, E.Z. Gusakov, and K. Niemi, Plasma Phys. Control. Fusion, 49, A167 (2007).

${ }^{11}$ G.R. Tynan, M.J. Burin, C. Holland, G. Antar, N. Crocker, and P.H. Diamond, Phys. Plasmas, 11, 5195 (2004).

${ }^{12}$ A.B. Altukhov, E.Z. Gusakov, M.A. Irzak, M. Kramer, B. Lorenz, and V.L. Selenin, Phys. Plasmas, 12, $022310(2005)$.

${ }^{13}$ A.I. Akheizer, V.S. Mikhailenko, V.V. Ol'shansky, and K.N. Stepanov, Plasma Physics Reports, 26, 575 (2000).

${ }^{14}$ C.S. Corr, N. Plihon, P. Chabert, O. Sutherland, and R.W. Boswell, Phys. Plasmas, 11, 4596 (2004).

${ }^{15}$ O. Sutherland, M. Giles, and R.W. Boswell, Phys. Rev. Lett., 94, 205002 (2005).

${ }^{16}$ T.H. Stix, Phys. Rev. Lett., 15, 878 (1965).

${ }^{17}$ D.G. Swanson, Plasma Waves, 2nd ed. (Institute of Physics, Philadelphia, 2003).

${ }^{18}$ E. Scime, R. Hardin, C. Biloiu, A.M. Keesee, and X. Sun, Phys. Plasmas, 14, 043505 (2007). 


\section{Chapter 8: Discussion}

The principle objective of this work was to determine if short wavelength fluctuations capable of heating ions are excited in helicon sources at the same plasma parameters for which anomalous ion heating has been observed in helicon sources. A portable $300 \mathrm{GHz}$, collective Thomson scattering (CTS) diagnostic, employing both quasi-optical propagation and a homodyne detection, was designed and installed on the HELIX source to measure short wavelength fluctuations; wavelengths on the order of 1 $\mathrm{mm}$. Such wavelengths are generally considered to be inaccessible by typical probes in high density helicon source plasmas.

The short wavelength fluctuations targeted for study by the CTS diagnostic were the Trivelpiece-Gould (TG) wave, or slow wave, believed to be one of the possible mechanisms for the efficient coupling of the rf power into the helicon plasma source. Measurements of the preferential heating of ions in the perpendicular direction at the plasma edge, shown to be correlated with the parameters expected to lead to excitation of the short wavelength, slow wave, motivated the search of the slow wave. Before the installation of the CTS diagnostic on HELIX, a verification of the homodyne detection system using a rotating diffraction grating to produce a Doppler shifted signal was employed.

After installation on HELIX, initial experiments with the CTS diagnostic identified a problem with a large amplitude, broadband noise signal peaked near 1-3 MHz. During one of the many studies designed to understand, and hopefully eliminate, the noise problem, the detector was damaged and had to be shipped to Germany for a lengthy repair. While the CTS diagnostic was shut down, a series of experiments to 
directly excite finite $k_{\perp}$ electrostatic waves propagating perpendicular to the magnetic field with an internal antenna were undertaken. While testing the antenna and an electrostatic double probe, spontaneously occurring excited waves capable of producing wave numbers measureable with the scattering diagnostic were found. The wave initially appeared to be parametrically driven by the rf pump wave for the helicon source.

With the electrostatic double probe, waves with perpendicular wave numbers in the wave number range measureable with the scattering diagnostic were investigated as a function of plasma parameters and radial location in the source. The spontaneously excited waves were divided into three groups; a low frequency (LF) group with frequencies in the range $0.2-1 \mathrm{MHz}$; a lower side band (LSB) group with frequencies $0.2-1 \mathrm{MHz}$ below the rf driving frequency; and an upper side band (USB) group with frequencies $0.2-1 \mathrm{MHz}$ above the rf driving frequency. Probe measurements indicated that the amplitude of the LF waves was largest for the same plasma conditions that resulted in enhanced ion heating in the plasma edge in previous experiments and that the waves were confined to the outer edge of the plasma; with peak amplitudes at $r \sim 4.5 \mathrm{~cm}$. For the same plasma conditions, the repaired CTS diagnostic observed statistically significant scattered wave power at frequencies of $f \sim 100 \mathrm{kHz}$ for a perpendicular wave number of $k_{\perp} \sim 89 \mathrm{rad} / \mathrm{cm}$. The CTS measurements were just barely above the noise levels of the diagnostic, but did show the same radial localization of wave power as the electrostatic double probe measurements.

The dispersion of the LF waves was similar to, but not completely consistent with expectations for ion-acoustic fluctuations. The radially localized, perpendicularly propagating LF waves do have perpendicular phase velocities capable of Landau 
damping on the ions, and are therefore an obvious candidate to explain the preferential perpendicular ion heating in the edge of helicon sources.

The dispersion of the LSB and USB waves are consistent with lower hybrid fluctuations (because of their oppositely directed phase and group velocities), and have phase velocities approaching the perpendicular electron thermal velocity. These sideband waves are an obvious candidate for the electron heating observed at the same radial locations where the fluctuations had the largest amplitudes.

The biggest challenge to the success of the CTS diagnostic is the broadband noise that seems to be intrinsic in the source. The broad noise spectrum peaked near 1-3 MHz and is large enough to beat with the rf frequency and produce the noise throughout the entire frequency range identified as most likely for a slow wave scattering signal to appear. For this diagnostic to be capable of making measurements at these frequencies this issue must be resolved. For the system in the current configuration, it is recommended that in later experiments the detector be moved to be directly inline with the "T" chamber, so that the scattered light collected does not need to be reflected off a Mylar sheet. This will reduce the thickness of the Mylar sheet, thereby increasing the amount of scattered light for mixing in the detector. While the rotating diffraction grating provided evidence that the CTS diagnostic could observe a Doppler shifted signal, it would be worth investigating the scattering from piezoelectric transducer generated acoustic waves in a block of HDPE similar to the scattering experiments described in Ref. [1] and Ref. [2]. This would allow for determining the mixer-detector response to scattered signals by varying the acoustic wave amplitude. It would also be worthwhile to 
continue the internally excited wave experiments to provide a controlled scattering "target" for the CTS diagnostic.

In light of the measurements with the electrostatic probe, it may be best to change the scattering geometry for the CTS system to measure the smaller wave number fluctuations which were observed to have fluctuation amplitudes much larger than those at larger wave numbers. As long as the beam divergence angle of the interaction beam is smaller than the angle of scattering, a much simpler scattering geometry could result in the measurement of a scattered signal corresponding to fluctuations measured with the electrostatic probe. 


\section{Chapter 8 References}

${ }^{1}$ J.F. Valley and R.E. Slusher, Rev. Sci. Instrum. 54, 1157 (1983).

${ }^{2}$ H. Park, C.X. Yu, W.A. Peebles, N.C. Luhmann Jr., and R. Savage, Rev. Sci. Instrum., 53, 1535 (1982). 


\section{Chapter 9: Conclusions}

A new, portable, $300 \mathrm{GHz}$ based, coherent Thomson scattering (CTS) diagnostic, employing quasioptical propagation and homodyne detection, has been designed and installed on the HELIX helicon source to non-perturbatively measure electrostatic fluctuations with wavelengths on the order of $1 \mathrm{~mm}$. Although the scattering diagnostic's detection capabilities were severely limited by broadband noise and rf pickup in the 1-10 MHz range, a low frequency $(f \sim 130 \mathrm{kHz})$ scattered signal with a large perpendicular wave number, $k_{\perp} \sim 89 \mathrm{rad} / \mathrm{cm}$, was detected and characterized as a function of radial location in the plasma. The scattered signal was localized in the plasma edge and the scattered amplitude was largest for $r \sim 4.5 \mathrm{~cm}$. Independent corroboration of the CTS measurements was obtained with a movable two-tip electrostatic probe. Analysis of the probe data identified strong, broad spectrum (in frequency and wave number) electrostatic waves in the same radial region, in the same frequency range, and in the same wave number range.

Given that the CTS diagnostic succeeded in measuring short wavelength electrostatic fluctuation in the plasma edge, it is possible to calculate an upper bound on the amplitude of any "slow" waves in the plasma edge at the rf driving frequency. Density fluctuation amplitudes $\left(\tilde{n} / n_{0}\right)$ of $10 \%$ in the measured edge plasma with a density of $10^{12} \mathrm{~cm}^{-3}$ would have been detectable above the troublesome noise in the $1-$ $10 \mathrm{MHz}$ range. Thus, the CTS measurements reported in this work are consistent with the absence of large amplitude, $>10 \%$, "slow" wave, electrostatic, fluctuations in the plasma edge. Elimination of the broadband noise is clearly needed to place a stronger constraint on the possible "slow" wave fluctuations at large wave number in the plasma edge. 
From the detailed probe measurements of the fluctuations in the $\sim 100 \mathrm{kHz}$ range, it appears that these waves have sufficiently small phase velocities and are at small enough wavelengths that they could interact with the ions. Sideband waves at frequencies just above and just below the rf frequency have phase velocities small enough to interact with the electrons and significant increases in electron temperature were observed at the same radial location as the peak in the wave amplitudes. The rf side band fluctuations (LSB and USB) have characteristics consistent with electrostatic lower hybrid waves due to their oppositely directed group and phase velocities. The low frequency fluctuations were tentatively identified as ion-acoustic fluctuations, but their phase velocity was inconsistent with expectations given the known electron temperature of the plasma. It was suggested that the finite size of the tips of the electrostatic probe might be responsible for the discrepancy between the measured and expected ion acoustic phase speeds.

Although the fluctuations were initially believed to be parametrically excited, detailed measurements of the wave number spectrum clearly showed that while the frequency matching condition is satisfied by the high frequency sideband waves with the rf driving wave and the low frequency wave, the wave number matching condition is not satisfied. The decrease in spectral power with increasing perpendicular wave number of the wave number spectrum of the low frequency fluctuations depended strongly on the plasma parameters and was much steeper that what would be expected for an inertial range. Thus, the wave number spectral measurements are consistent with active dissipation or damping of the fluctuations at the measured spatial scales. 


\section{Appendix A: Pressure Calibration Data}

The pressure gauge calibration for argon and helium was recently performed because of the new gas inlet at the center of HELIX. Calibrations were accomplished for both the center and end gas feeds and with either the end gate valve open and closed. Although the Balzers gauge is gas dependant thus requiring correction to the real pressure, the raw (uncorrected) Balzer pressure gauges are reported. To correct the Balzers gauge pressures the calibration curves from the operating manual must be used.
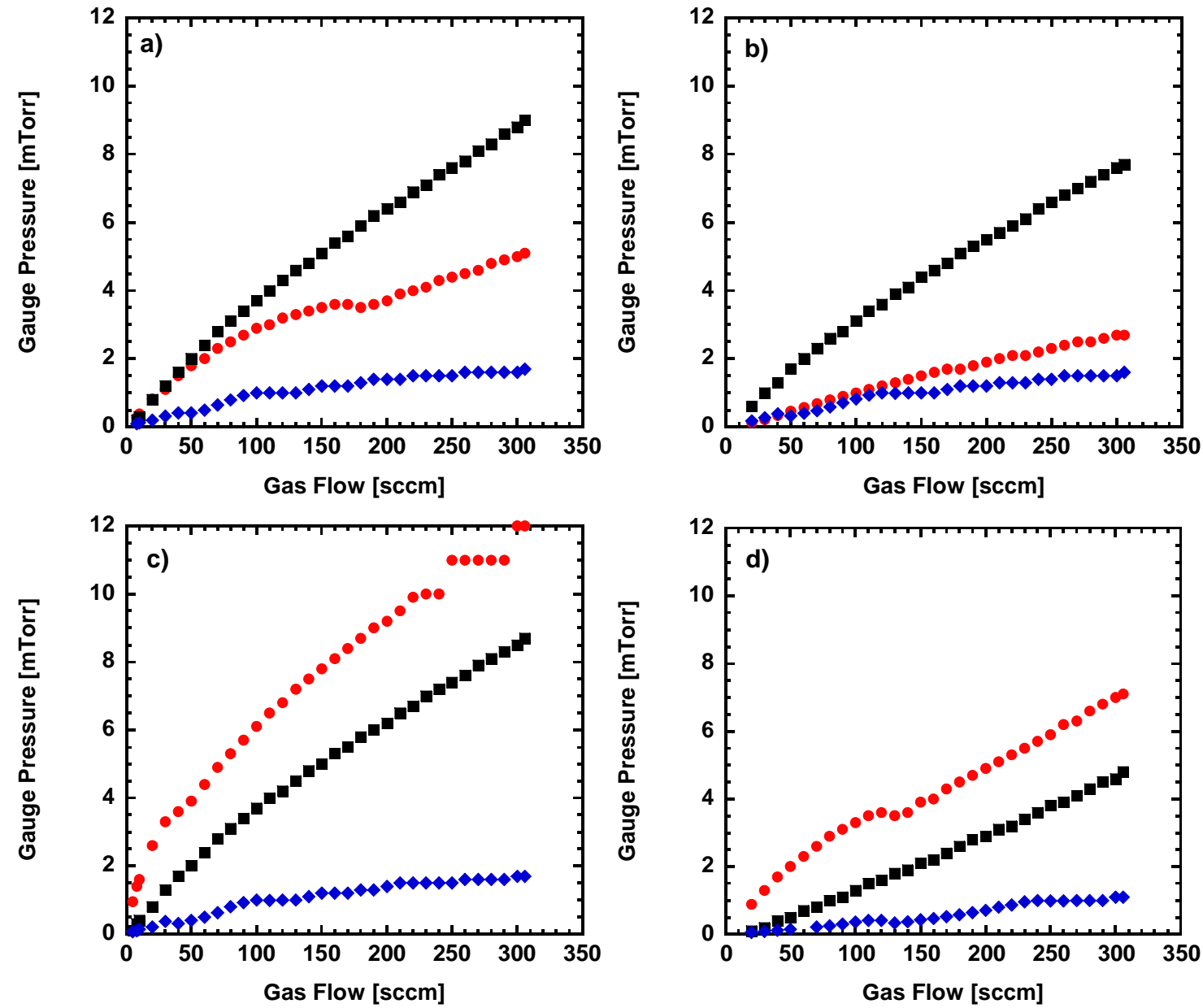

Figure A.1 Full range pressure gauge calibration for argon: (a) center feed and gate valve closed, (b) center feed and gate valve open, (c) end feed and gate valve closed, and (d) end feed and gate valve open. Red circles (๑) are the HELIX Balzer gauge, black squares (ם) are the HELIX Baratron gauge, and the blue diamonds $(\diamond)$ are the LEIA Balzer gauge. 

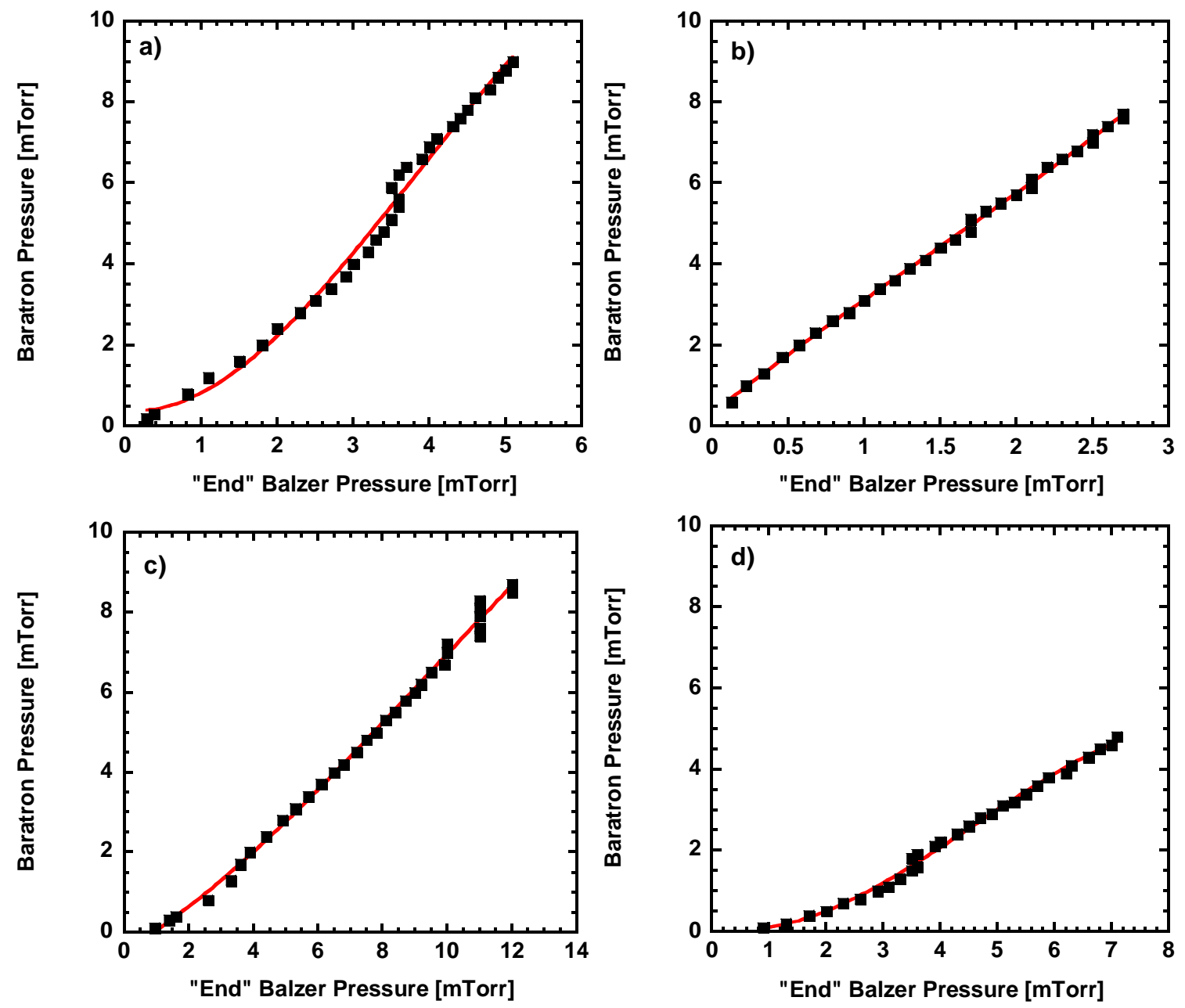

Figure A.2 Baratron and uncorrected "end" Balzers pressure gauge calibration for argon in HELIX: (a) center feed and gate valve closed, (b) center feed and gate valve open, (c) end feed and gate valve closed, and (d) end feed and gate valve open.

The polynomial fits, as applied to the respective plots, are as follows:

$$
\begin{gathered}
P_{B}=0.39-0.17 P_{G}+0.66 P_{G}^{2}-0.05 P_{G}^{3} \\
P_{B}=0.30+3.02 P_{G}-0.28 P_{G}^{2}-0.06 P_{G}^{3} \\
P_{B}=-0.47+0.47 P_{G}+0.04 P_{G}^{2}-0.001 P_{G}^{3} \\
P_{B}=0.11-0.24 P_{G}+0.25 P_{G}^{2}-0.02 P_{G}^{3}
\end{gathered}
$$

where $P_{G}$ is the Balzers uncorrected gauge pressure and $P_{B}$ is the Baratron pressure. 

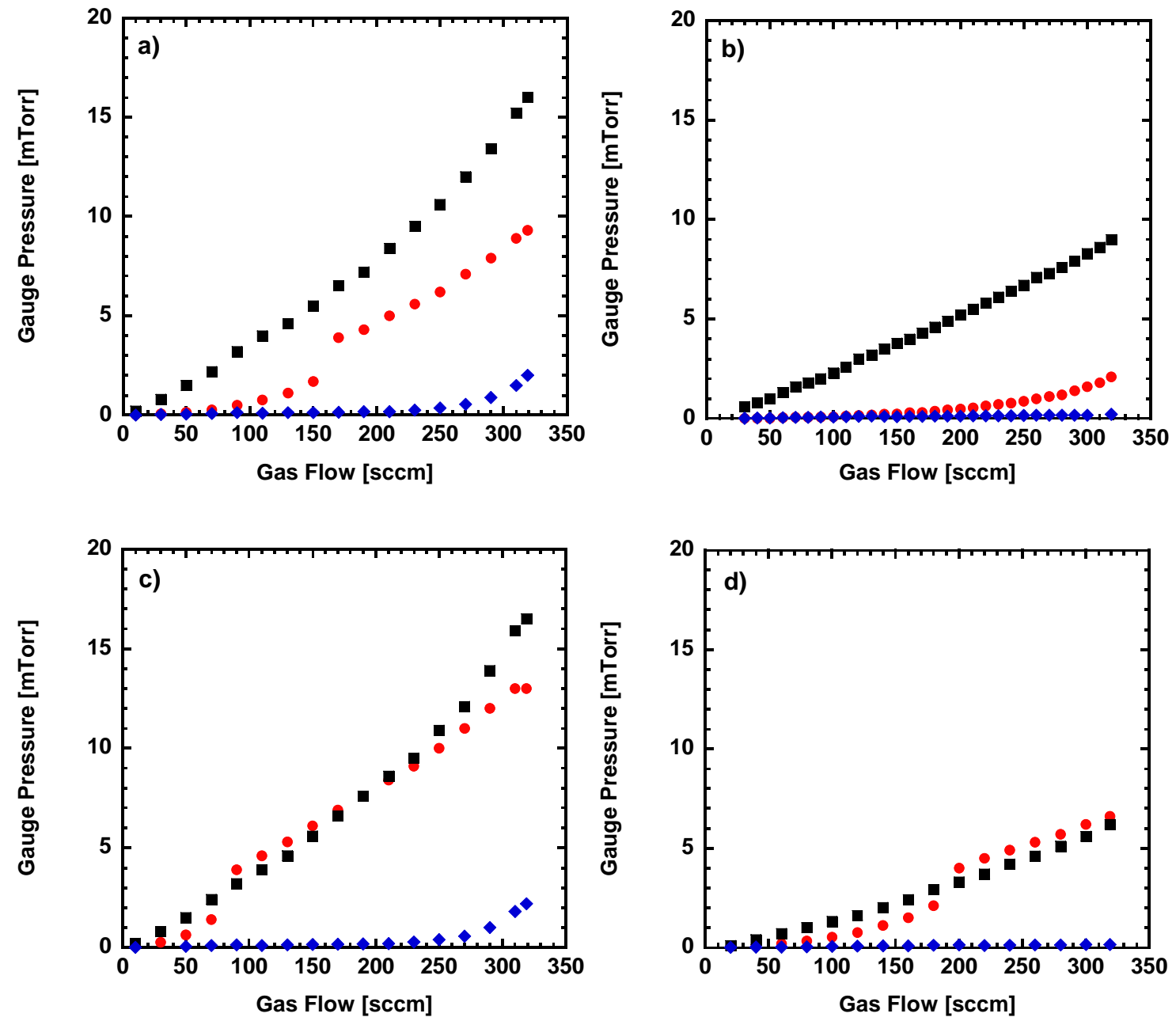

Figure A.3 Full range pressure gauge calibration for helium: (a) center feed and gate valve closed, (b) center feed and gate valve open, (c) end feed and gate valve closed, and (d) end feed and gate valve open. Red circles (๑) are the HELIX Balzer gauge, black squares (ם) are the HELIX Baratron gauge, and the blue diamonds $(\diamond)$ are the LEIA Balzer gauge. 

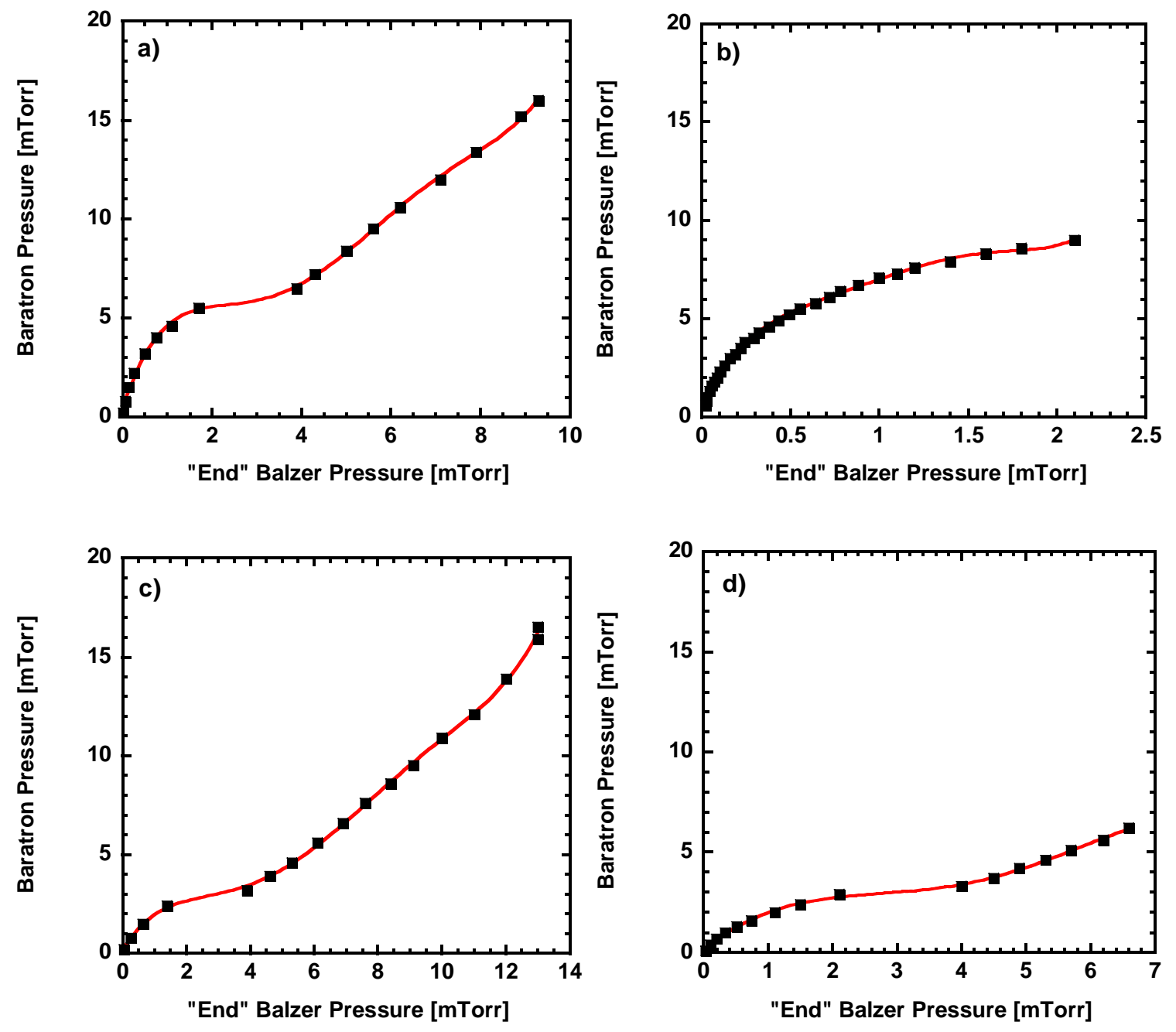

Figure A.4 Baratron and uncorrected "end" Balzers pressure gauge calibration for helium in HELIX: (a) center feed and gate valve closed, (b) center feed and gate valve open, (c) end feed and gate valve closed, and (d) end feed and gate valve open.

The polynomial fits, as applied to the respective plots, are as follows:

$$
\begin{aligned}
& P_{B}=0.38+7.15 P_{G}-3.75 P_{G}^{2}+0.91 P_{G}^{3}-0.09 P_{G}^{4}+0.004 P_{G}^{5} \\
& P_{B}=0.47+19.47 P_{G}-31.37 P_{G}^{2}+29.68 P_{G}^{3}-13.58 P_{G}^{4}+2.33 P_{G}^{5} \\
& P_{B}=0.14+2.70 P_{G}-1.09 P_{G}^{2}+0.22 P_{G}^{3}-0.02 P_{G}^{4}+0.0005 P_{G}^{5} \\
& P_{B}=0.14+2.62 P_{G}-0.86 P_{G}^{2}+0.08 P_{G}^{3}-0.008 P_{G}^{4}+0.001 P_{G}^{5}
\end{aligned}
$$

where $P_{G}$ is the Balzers uncorrected gauge pressure and $P_{B}$ is the Baratron pressure. 


\section{Appendix B: Cold Plasma Dispersion Relation Modeling Code}

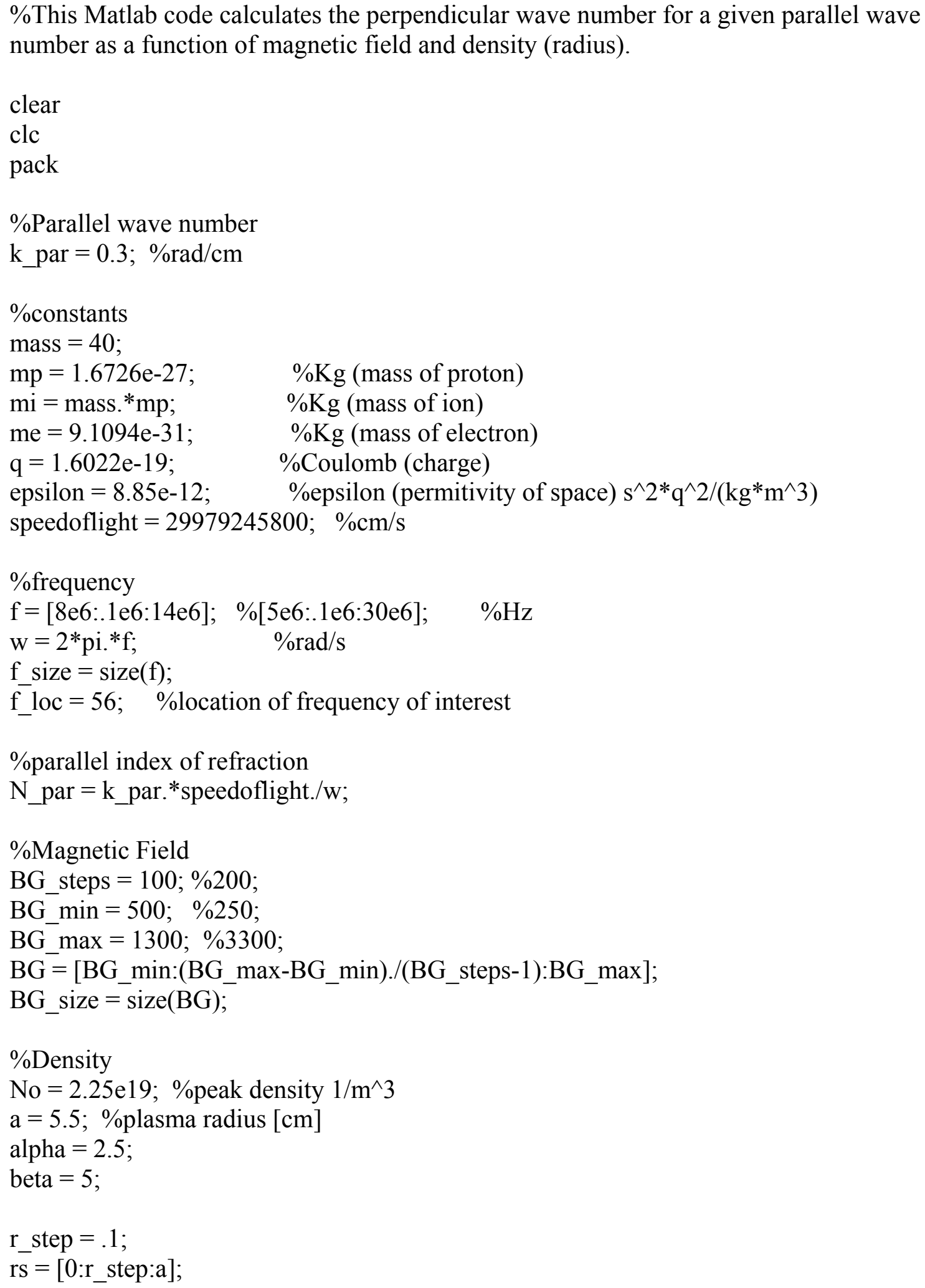




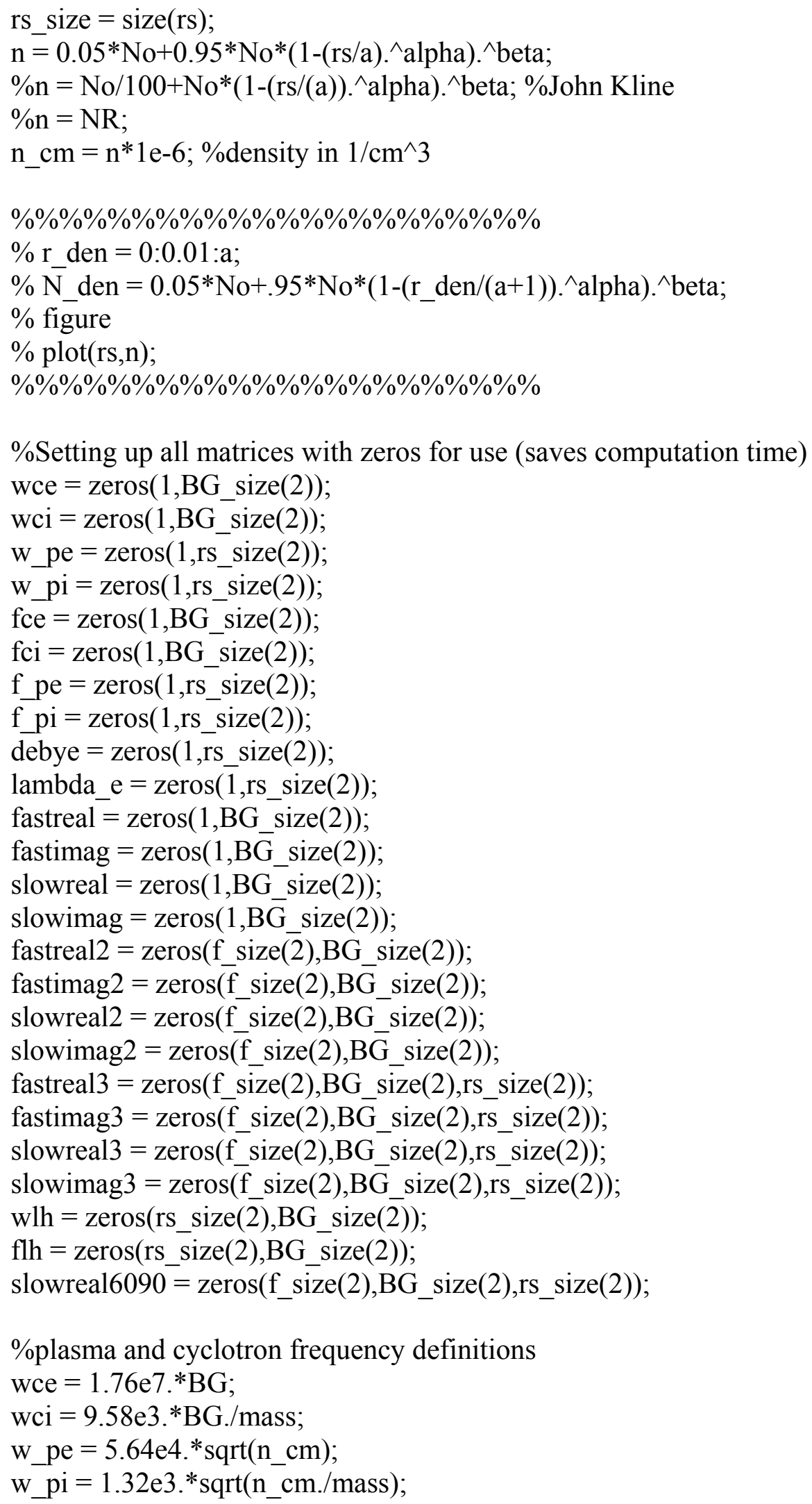




$$
\begin{aligned}
& \text { fce }=\text { wce. } /(2 * \text { pi }) \\
& \text { fci }=\text { wci./(2*pi); } \\
& \text { f_pe }=\text { w_pe./(2*pi); } \\
& \text { f_pi }=\text { w_pi./(2*pi); }
\end{aligned}
$$

\section{$\%$ COLLISIONS}

collision $=1 ; \%$ if 1 collisions are on, if 0 collisions are off

$\mathrm{Ti}=.2 ; \quad \%[\mathrm{eV}]$

$\mathrm{Te}=3 ; \quad \%[\mathrm{eV}]$

debye $=743 *$ sqrt $($ Te./n_cm $) ; \%$ electron debye length $[\mathrm{cm}]$

lambda_e $=\log \left(12 *\right.$ pi $^{*}$ n_cm.*(debye.^3) $) ; \%$ Coulomb Logarithm

Pressure $=8 \mathrm{e}-3 ; \% 6.7 \mathrm{e}-3 ; \%[$ Torr $]$

$\mathrm{Nn}=(2.6868 \mathrm{e} 19 . *$ Pressure. $/ 760) . *(0.02 / 0.04) ; \%$ Neutral density based on pressure \& slightly above room temp

Vthi $=9.79 \mathrm{e} 5 .^{*}$ sqrt(Ti./mass); \%Ion Thermal velocity $[\mathrm{cm} / \mathrm{s}]$

Vthe $=4.19 \mathrm{e} 7 * \operatorname{sqrt}(\mathrm{Te}) ; \%$ Electron Thermal velocity $[\mathrm{cm} / \mathrm{s}]$

for $\mathrm{x}=1$ :rs_size $(2)$

$$
\begin{aligned}
& \text { wpe }=\mathrm{w} \_ \text {pe }(x) \\
& \text { wpi }=\text { w_pi }(x) ; \\
& \text { ncm }=\text { n_cm }(x)
\end{aligned}
$$$$
\text { lambda }=\text { lambda_e(x); }
$$

$$
\begin{aligned}
& \text { if }(\text { collision }==0) \\
& \text { Nui }=0 ; \\
& \text { Nue }=0 ; \\
& \quad \text { else }
\end{aligned}
$$$$
\text { Nui }=4.8 \mathrm{e}-8 .{ }^{*} \operatorname{sqrt}\left(\left(\mathrm{Ti} .{ }^{\wedge} 3\right) . / \mathrm{mass}\right) .{ }^{*} \mathrm{ncm} .{ }^{*} \text { lambda. }{ }^{*} \mathrm{Ti}^{\wedge}{ }^{\wedge}(-(3 / 2))+(\mathrm{Nn}-\mathrm{ncm}) .{ }^{*} 1 \mathrm{e}-
$$
15.*9.79e5.*sqrt(Ti./mass);

Nue $=\left(\right.$ Nn-ncm). $* 1$ e-15.*4.19e7. $* \operatorname{sqrt}(\mathrm{Te})+2.91 \mathrm{e}-6 .{ }^{*}$ ncm. ${ }^{*}$ lambda. ${ }^{*}$ Te. ${ }^{\wedge}(-(3 / 2))$; $\% 1$ e- $3 * 2 *$ pi*8e6;

end

for $\mathrm{t}=1$ :f_size $(2) \%$ frequency set (rows)

for $\mathrm{v}=1: \mathrm{BG} \_$size $(2) \% \mathrm{~B}$ field set (columns)

\%Lower Hybrid Frequency

$\mathrm{wlh}(\mathrm{x}, \mathrm{v})=1 . / \operatorname{sqrt}\left(\left(1 . /\left(\mathrm{wce}(\mathrm{v}) .^{*} \mathrm{wci}(\mathrm{v})\right)\right)+\left(1 . /\left(\mathrm{wpi} .{ }^{\wedge} 2+\mathrm{wci}(\mathrm{v}) .{ }^{\wedge} 2\right)\right)\right)$;

$\%$ rows are radius(density) columns are $\mathrm{B}$

\%Cold Plasma Dielectric Tensor Elements

eps1 $=1+\left(\right.$ wpe. $\left.{ }^{\wedge} 2 .^{*}\left(1+\left(\mathrm{i} \cdot{ }^{*} \mathrm{Nue} . / \mathrm{w}(\mathrm{t})\right)\right)\right) . /\left(\mathrm{wce}(\mathrm{v}) . \wedge 2-\mathrm{w}(\mathrm{t}) .^{\wedge} 2{ }^{*}(1+(\mathrm{i} \cdot * \mathrm{Nue} . / \mathrm{w}(\mathrm{t}))) . \wedge 2\right)+\ldots$ (wpi.^2.*(1+(i.*Nui./w(t))))./(wci(v).^2-w(t).^2.*(1+(i.*Nui./w(t))).^2);

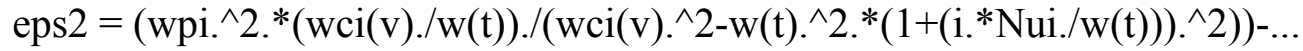
(wpe. $\left.{ }^{\wedge} 2 .^{*}(\mathrm{wce}(\mathrm{v}) \cdot / \mathrm{w}(\mathrm{t})) \cdot /\left(\mathrm{wce}(\mathrm{v}) .^{\wedge} 2-\mathrm{w}(\mathrm{t}) \cdot{ }^{\wedge} 2 \cdot^{*}\left(1+\left(\mathrm{i} .^{*} \mathrm{Nue} . / \mathrm{w}(\mathrm{t})\right)\right) \cdot{ }^{\wedge} 2\right)\right)$;

eps3 = 1-wpe.^2./(w(t).^2.*(1+(i.*Nue./w(t))))-wpi.^2./(w(t).^2.*(1+(i.*Nui./w(t)))); 
$\%$ Values used in dispersion relation (Cho 2000, Kline)

$$
\begin{aligned}
& \text { alpha1 = eps1-(N_par(t).^2)-(eps2.^2./eps } 1) \\
& \text { beta1 }=\text { eps3.*(1-(N_par(t).^2./eps } 1)) \\
& \text { gamma } 1=\mathrm{N} \_ \text {par(t).*eps3.*eps2./eps } 1 ; \\
& \text { delta1 = N_par(t).*eps2./eps1; }
\end{aligned}
$$

$\%$ Calculating the dispersion relation, and determining the $\mathrm{k} \_$perp solution to the dispersion relation

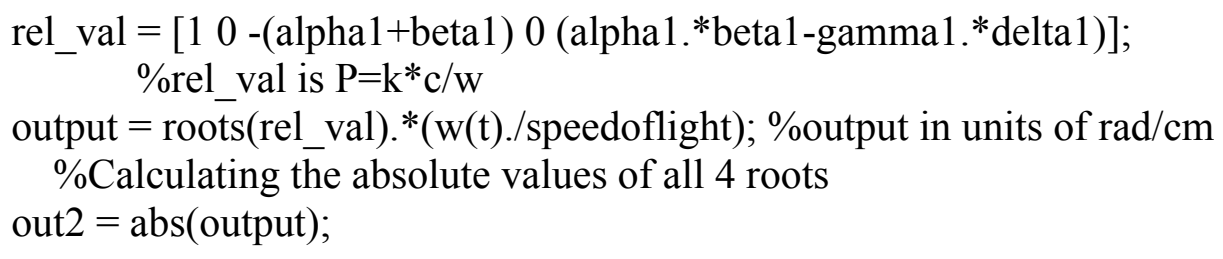

$\%$ Separation of the fast and slow roots and the index location of

$\%$ those roots based on the absolute value of each $\mathrm{k} \_$perp,

$\%$ WITHOUT assuming (for example) root 1 is

$\%$ always the fast wave and root 3 is always the slow wave.

[outslow slowloc] $=\max ($ out 2$)$;

[outfast fastloc] $=\min ($ out 2$)$;

$\%$ Roots as a function of Magnetic Field (Fixed frequency,

$\%$ and radius (density))

$\operatorname{slowreal}(\mathrm{v})=\operatorname{abs}(\operatorname{real}(\operatorname{output}($ slowloc $)))$;

slowimag $(\mathrm{v})=\operatorname{abs}(\operatorname{imag}($ output $($ slowloc $)))$;

fastreal $(\mathrm{v})=\operatorname{abs}(\operatorname{real}($ output $($ fastloc $)))$;

fastimag $(\mathrm{v})=\operatorname{abs}(\operatorname{imag}(\operatorname{output}($ fastloc $)))$;

end

$\%$ Roots after being calculated for Magnetic Field, as a function

$\%$ of frequency (Fixed radius (density))

fastreal2 $(\mathrm{t},:)=$ fastreal;

fastimag2 $(\mathrm{t},:)=$ fastimag;

slowreal2 $(\mathrm{t},:)=$ slowreal;

slowimag2 $(\mathrm{t},:)=$ slowimag;

end

$\% 3 \mathrm{D}$ matrix of $\mathrm{k}$ perp, rows $=$ frequency, columns $=$ magnetic field, $\%$ depth $=$ radius(density)

fastreal3 $(:, ;, \mathrm{x})=$ fastreal 2 ;

fastimag $3(:, ;, \mathrm{x})=$ fastimag 2 ;

slowreal3(:,:,x) = slowreal2;

slowimag3(:,:,x) = slowimag2; 
end

\%Normalizing the $\mathrm{k}$ _perp solutions to the Ion Thermal Velocity

for $\mathrm{z}=1$ :f_size $(2)$

Norm_slowreal3(z,:,:) = slowreal3(z,:,:).*Vthi./w(z);

Norm_slowimag3(z,:::) = slowimag3(z,:,:).*Vthi./w(z);

end

\%This section pulls out the maximum $\mathrm{k} \_$perp and radial location for each frequency and magnetic field in radius, to see the maximum $\mathrm{k}$ _perp through the entire radius

for $z=1$ :rs_size(2) \%radius [density] (depth)

for $\mathrm{t}=1$ :f_size (2) \%frequency set (rows)

for $\mathrm{v}=1: \mathrm{BG}$ _size $(2) \% \mathrm{~B}$ field set (columns)

[slowreal_max $(\mathrm{t}, \mathrm{v})$ slowreal_max_loc $(\mathrm{t}, \mathrm{v})]=\max (\operatorname{slowreal} 3(\mathrm{t}, \mathrm{v}, \mathrm{:}))$;

[slowimag_max $(\mathrm{t}, \mathrm{v})$ slowimag_max_loc $(\mathrm{t}, \mathrm{v})]=\max (\operatorname{slowimag} 3(\mathrm{t}, \mathrm{v},:))$;

[Norm_slowreal_max $(\mathrm{t}, \mathrm{v})$ Norm_slowreal_max_loc $(\mathrm{t}, \mathrm{v})]=$

$\max ($ Norm_slowreal3 $(\mathrm{t}, \mathrm{v}, \mathrm{i}))$;

[Norm_slowimag_max $(\mathrm{t}, \mathrm{v})$ Norm_slowimag_max_loc $(\mathrm{t}, \mathrm{v})]=$

$\max ($ Norm_slowimag3(t,v,:));

$\% \quad$ [fastreal_max $(\mathrm{t}, \mathrm{v})$ fastreal_max $\operatorname{loc}(\mathrm{t}, \mathrm{v})]=\max (\operatorname{fastreal3}(\mathrm{t}, \mathrm{v},:))$;

$\% \quad[$ fastimag_max $(\mathrm{t}, \mathrm{v})$ fastimag_max_loc $(\mathrm{t}, \mathrm{v})]=\max (\operatorname{fastimag} 3(\mathrm{t}, \mathrm{v}, \mathrm{i}))$;

$\%$ This if statement pulls out all the $\mathrm{k}$ _perp values from slowreal3

\%ranging from 60 to 90 , which correspond to the measureable

$\%$ range of the mm-wave scattering diagnostic

if $(($ slowreal3 $(\mathrm{t}, \mathrm{v}, \mathrm{z})>=60) \& \&(\operatorname{slowreal3}(\mathrm{t}, \mathrm{v}, \mathrm{z})<=90))$

slowreal6090 $(\mathrm{t}, \mathrm{v}, \mathrm{z})=$ slowreal3 $(\mathrm{t}, \mathrm{v}, \mathrm{z})$;

end

end

end

end

\%Lower Hybrid Frequency [MHz]

$\mathrm{flh}=($ wlh./(2* pi) $) . / 1 \mathrm{e} 6$;

$\%$ This section pulls out the $\mathrm{k} \_$perp's for a specific frequency,

$\%$ as a function of $\mathrm{B}$ and radius. This shows for the given

$\%$ frequency the radial location of the allowable $\mathrm{k}$ 's as a function of $\mathrm{B}$.

$\%$ f_loc $=19$;

$\mathrm{k}=\overline{1}$;

for $\mathrm{i}=1$ :rs_size $(2)$

for $\mathrm{j}=1: \mathrm{BG}$ size $(2)$

slowreal_radius $(\mathrm{i}, \mathrm{j})=\operatorname{slowreal3}\left(\mathrm{f} \_l o c, \mathrm{j}, \mathrm{i}\right)$; 
slowimag_radius $(\mathrm{i}, \mathrm{j})=\operatorname{slowimag} 3\left(\mathrm{f} \_\right.$loc $\left., \mathrm{j}, \mathrm{i}\right)$;

Norm_slowreal_radius $(\mathrm{i}, \mathrm{j})=$ Norm_slowreal3(f_loc, $\mathrm{j}, \mathrm{i})$;

slowreal6090_radius $(\mathrm{i}, \mathrm{j})=\operatorname{slowrea16090}\left(\mathrm{f} \_l o c, \bar{j}, \mathrm{i}\right)$;

$\%$ This if statement creates a subset of Magnetic Field and radius

$\%$ values for a given frequency range (based on the frequency resolution)

$\%$ around the chosen frequency defined by $\mathrm{f}$ _loc above. Basically what

$\%$ this is doing is for the chosen frequency, say $\mathrm{f}=9.5 \mathrm{MHz}$ with

$\%$ frequency resolution of $0.5 \mathrm{MHz}$, it is checking to see if there is

$\%$ a lower hybrid frequency in this range, and marking the radius and

$\%$ magnetic field values, so as to plot the resonance layer as a

$\%$ function of the radius and magnetic field

if $\left(\left(\mathrm{f}\left(\mathrm{f} \_l o c-1\right) / 1 \mathrm{e} 6<=\mathrm{flh}(\mathrm{i}, \mathrm{j})\right) \& \&\left(\mathrm{f}\left(\mathrm{f} \_\right.\right.\right.$loc +1$\left.\left.) / 1 \mathrm{e} 6>=\mathrm{flh}(\mathrm{i}, \mathrm{j})\right)\right)$

r_flh(k,:) $=[\mathrm{BG}(\mathrm{j}) \mathrm{rs}(\mathrm{i})] ;$ \%radius/B field of the lower hybrid frequency resonance $\mathrm{k}=\mathrm{k}+1$;

end

end

end 


\section{Appendix C: Alignment and General Calibration Guidelines for the 300 GHz Scattering Diagnostic}

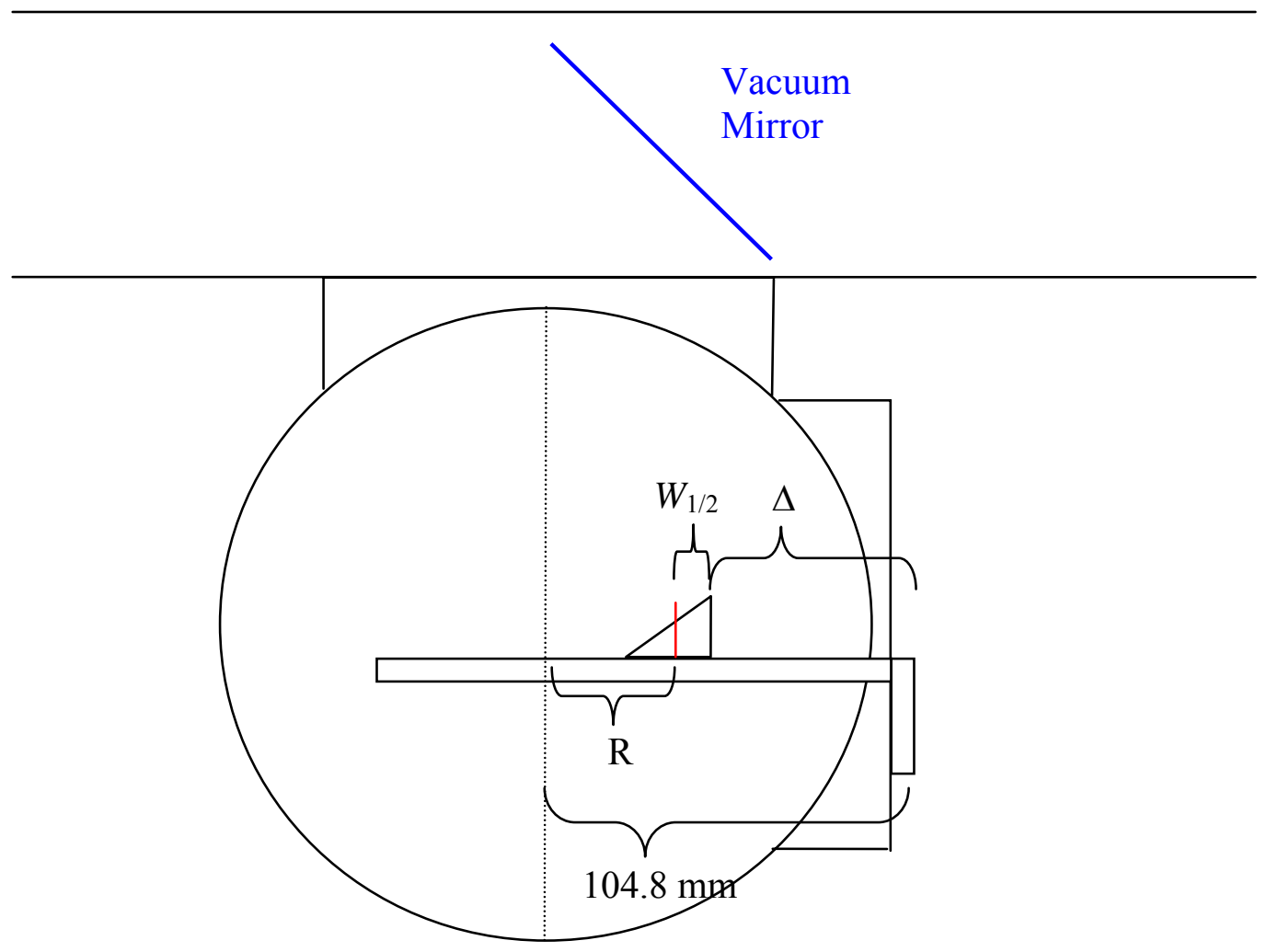

Figure C.1 HELIX chamber geometry and dimension definitions for the alignment wedges.

\section{$\underline{\text { Alignment Guidelines }}$}

1. Align the laser pointer beam across the center of the HELIX chamber using the window alignment grids.

2. Alignment of the collection mirror via the laser pointer.

a. Drive the vacuum mirror to a point close to where the bottom of the mirror is near the bottom edge, closest to the opening of the " $\mathrm{T}$ " chamber. 
b. Insert the $90^{\circ}$ alignment wedge until the center of the beam is just inside of the " $T$ " chamber.

c. Tilt the vacuum mirror until you can see the beam hitting the bottom most part of the mirror.

d. Move the wedge to the calculated distance where the beam should be centered coming out the side of the " $\mathrm{T}$ " chamber.

e. Adjust the micrometer until the beam is exiting the " $\mathrm{T}$ " chamber center.

i. NOTE: Due to hysteresis in the structure, it is advantageous to either push or pull on the mirror consistently with the micrometer. In my experience, it has been beneficial to use the pulling method. If pushing is required to obtain a different angle, go past by about 30-50 thousandths on the micrometer, and pull back the desired angle setting.

f. Reset the alignment wedge to the location in step 2c.

i. If the beam is no longer at the bottom of the mirror, move the vacuum mirror until the mirrors bottom is positioned back over the beam again.

ii. Repeat steps $2 \mathrm{~d}$ and $2 \mathrm{f}$ until both conditions are met simultaneously.

iii. Record the setting of the micrometer as this will be the "zero" reference location.

iv. Repeat steps $2 \mathrm{a}$ through $2 \mathrm{f}$ for each alignment wedge until all four angles on the vacuum mirror have been calibrated. 
Table C.1 Vacuum mirror collection angle and mirror location.

\begin{tabular}{|c|c|c|c|}
\hline $\begin{array}{c}\text { Collection Angle } \\
{[\mathrm{deg}]}\end{array}$ & $\begin{array}{c}\text { Velmex ‘0’ Mirror } \\
\text { Location }[\mathrm{cm}]\end{array}$ & $\begin{array}{c}\text { Wedge Location } \\
{[\mathrm{mm}]}\end{array}$ & Micrometer Setting \\
\hline 90 & 10 & 98.8 & 700 \\
\hline 80 & 9.7 & 123 & 565 \\
\hline 70 & 9.3 & 150 & 455 \\
\hline 60 & 9.0 & 150 & 350 \\
\hline
\end{tabular}

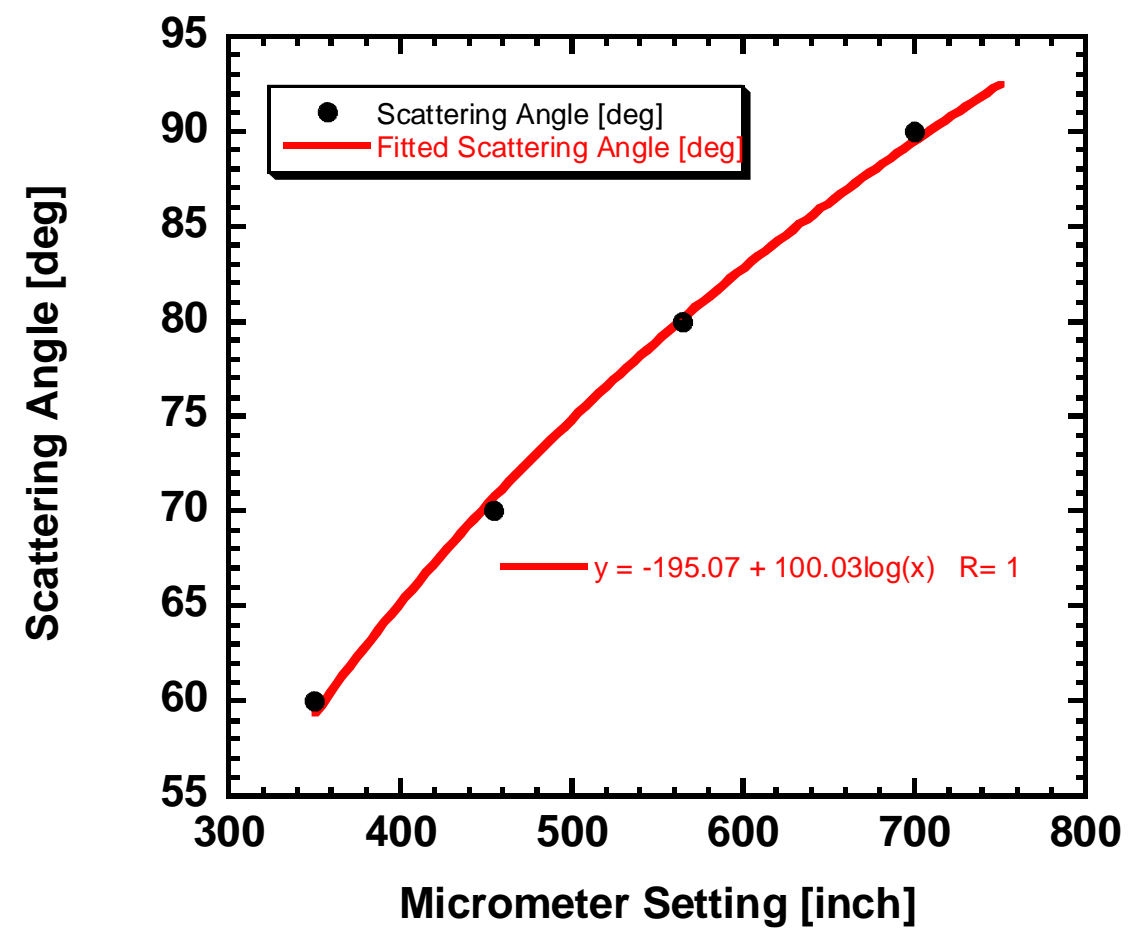

Figure C.2 Vacuum mirror micrometer calibration data and fit for determining the collection angle.

3. Plot the micrometer settings as a function of the scattering angle, and fit the data to obtain the micrometer values for the entire range of possible scattering angles.

4. Align the lenses, beam mixer, and beam splitter working from the detector backwards toward the source.

5. Initial alignment is complete.

\section{Calibration Guidelines}

1. Block the reference beam by using a piece of Eccosorb. 
2. Turn on $300 \mathrm{GHz}$ source and detector as outlined in " $300 \mathrm{GHz}$ Detector Initialization" procedures.

3. Position the vacuum collection mirror for the desired collection angle at the corresponding Velmex "zero" location.

4. Insert corresponding alignment wedge back to where the laser pointer beam was centered $1 / 2$ " above the " $T$ " chamber centerline.

a. NOTE: There should be some signal on the Spectrum Analyzer or something maybe wrong.

b. Slight adjustment of the beam mixer tilt or detector horn location may be needed to maximize the signal.

c. Slight adjustment of the injection mirrors may be done for signal maximization, but is not recommended.

5. Move the alignment wedge to about $2 \mathrm{~cm}$ from the slider mount at the edge of the vacuum chamber.

a. In steps of $\sim 1 \mathrm{~mm}$ to $5 \mathrm{~mm}$ (user discretion), move the wedge across the vacuum chamber diameter, recording the peak signal amplitude and distance from the back of the alignment wedge to the slider mount.

b. Checks to determine if the collection area across the plasma is maintained may be accomplished by moving the collection mirror and repeating step 5 a for different locations in the vacuum chamber.

6. Repeat step 5 for all 4 alignment wedges.

7. Main calibration is complete. 
8. Since the beam is now maximized through the Injection/Collection beam line, alignment of the reference beam line may now be performed.

a. Block the injection beam line with eccosorb.

b. Maximize the reference signal by ONLY adjusting the mirrors in the reference beam line (i.e. after the initial beam splitter, and before the beam mixer).

9. Linearization of the spectrum analyzer values may be calculated by using $P=10^{\left(\frac{x-30}{10}\right)}[$ Watt] where $\mathrm{x}$ is in $\mathrm{dBm}$.

10. The radial location is determined by $R=104.8-\left(\Delta+W_{1 / 2}\right)[\mathrm{mm}]$ where $\Delta$ is the distance from the back of the alignment wedge to the slider mount and $W_{1 / 2}$ is the $1 / 2$ width of the specific alignment wedge. (See Figure 1)

Table C.2 Vacuum mirror alignment wedge widths.

\begin{tabular}{|c|c|}
\hline Scattering Wedge $[\mathrm{deg}]$ & $1 / 2$ Wedge Width $\left(W_{1 / 2}\right)[\mathrm{mm}]$ \\
\hline 90 & 12.7 \\
\hline 80 & 15.15 \\
\hline 70 & 18.15 \\
\hline 60 & 22 \\
\hline
\end{tabular}

11. Determining the measured collection beam width (waist) is accomplished by fitting the linearized values to a Gaussian $f(x)=A e^{\frac{-\left(x-x_{0}\right)^{2}}{2 \sigma^{2}}}$, where $A$ is the arbitrary amplitude, $\mathrm{x}_{0}$ is the offset peak location, and $\sigma$ is the variance used in calculating the waist size.

a. Waist size varies in definition so the three general values are defined as follows in relation to the variance:

i. Full width at half maximum $(\mathrm{FWHM})=\sqrt{8 \ln (2)} \sigma \approx 2.35 \sigma$ 
ii. Full width at $1 / e$ folding point $=\sqrt{8} \sigma \approx 2.83 \sigma$

iii. Full width at $1 / e^{2}$ folding point $=4 \sigma$

b. ZEMAX is not very explicit about their description or definition of the beam waist or beam width, although they are different entities. The code is set to using a Gaussian Apodization of 2.33 (John Heard defined), where $\mathrm{GA}=0$ is a uniform amplitude, $\mathrm{GA}=1$ has the amplitude at $1 / e$ and the intensity at $1 / e^{2}$.

i. The Gaussian source definition (pg. 349 of Zemax manual) says the beam size is the beam radius at the $1 / e^{2}$ point.

ii. The beam width defined as $\mathrm{W}_{\mathrm{x}}=2 \sigma_{\mathrm{x}}$ (pg. 544 of Zemax manual) is a reference to the $1 / e^{2}$ point as well. 


\title{
Appendix D: Spectral Density Calculation Code
}

\author{
*Program (LAB WINDOWS) : K spectrum analysis \\ *Writen By : John Kline with analysis routine by Paul Keiter \\ * Date Alpha version : 4/26/2000 \\ * 5/21/2008 Modified by E. Scime to allow for variable length record \\ * record analysis during file input process \\ * 6/30/2008 Modified by R. Hardin to allow for choosing the window \\ * functions and to import binary files \\ * This routine calculates $\mathrm{K}$ given two time series of data then puts the $\mathrm{K}$ in a $2 \mathrm{~d}$ \\ * array of freq vs K. \\ $*$

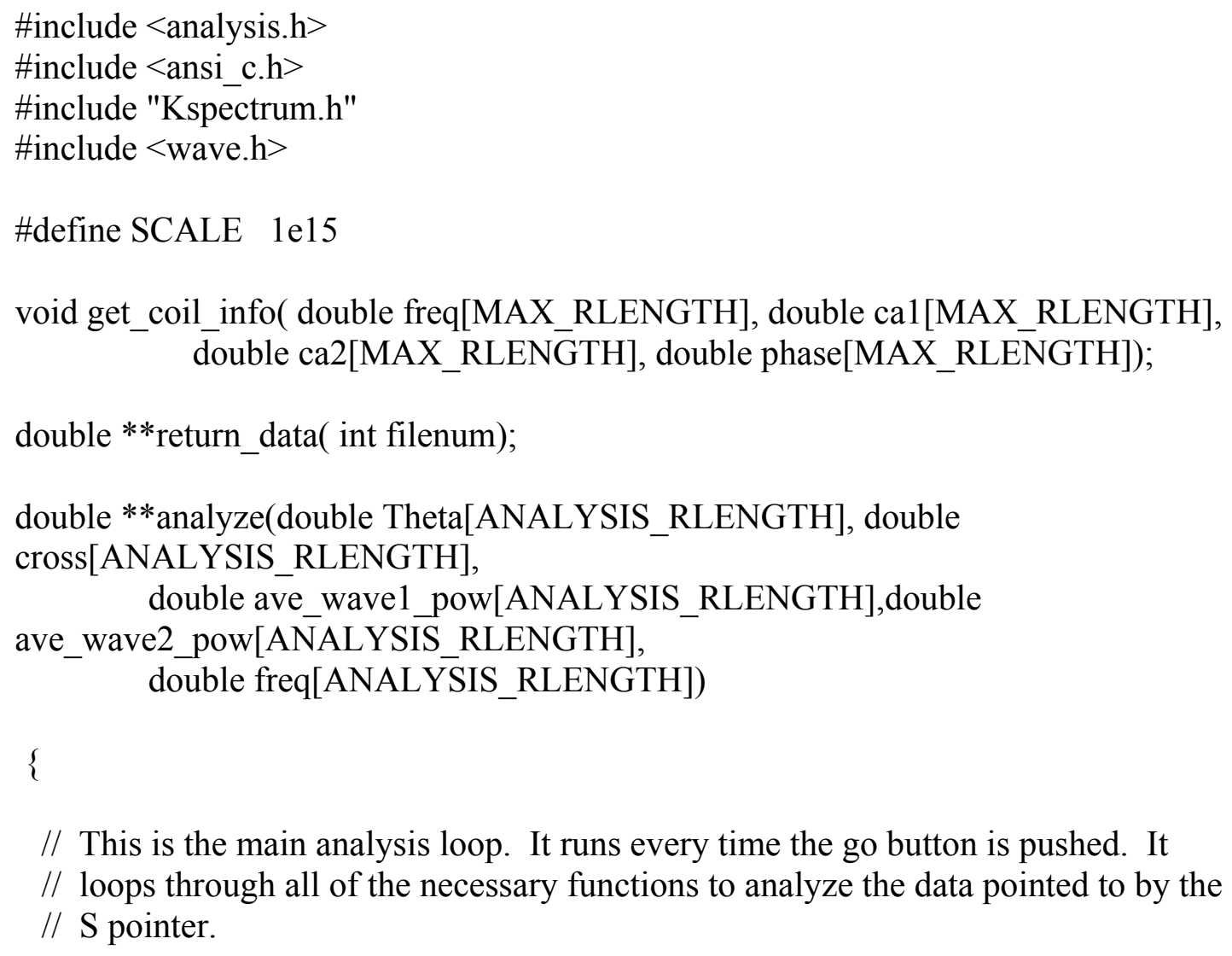

// This is the main analysis loop. It runs every time the go button is pushed. It

// loops through all of the necessary functions to analyze the data pointed to by the

// $\mathrm{S}$ pointer.

static double **wave1; // pointer to a $2 \mathrm{~d}$ array of time series for analysis

static double **wave2; // pointer to a $2 \mathrm{~d}$ array of time series for analysis static double **S;

double wavelout[ANALYSIS_RLENGTH]; // array used to pass a single time series to analysis routine 
double wave2out[ANALYSIS_RLENGTH]; / array used to pass a single time series to analysis routine
double srate;
// sampling rate variable
double dist;
// coil separation distance
int iterations;
// Number of time series to use in analysis
int rlength;
// Number of points in time series to analyze
int krlength;
// Number of points for K measuments
int kbins;
// Number of fft bins to combine for $\mathrm{K}$
static int cols 1 ;
// Number of columns in Time series file
static int cols2;
// Number of columns in Time series file
static int max_data_length1;
static int max_data_length2;
int $\mathrm{i}, \mathrm{j}, \mathrm{k}$;
// counters
int coilnum1;
int coilnum2;
int result $=0$;
int klambda_flag;
// generic result variable
static int old_rlength;
// flag to plot vs K or vs lambda
static int old_krlength;
// Stores old value of rlength before processing
// Stores old value of Krlength before processing

// get defalt values

GetCtrlVal (main_panel, PANEL_DRATE, \&srate);

GetCtrlVal (main_panel, PANEL_ITER, \&iterations);

GetCtrlVal (main_panel, PANEL_RLENGTH, \&rlength);

GetCtrlVal (main_panel, PANEL_KBINS, \&kbins);

GetCtrlVal (main_panel, PANEL_COILNUM1, \&coilnum1);

GetCtrlVal (main_panel, PANEL_COILNUM2, \&coilnum2);

// calculate number of $\mathrm{K}$ values need for analysis

krlength = kbins; $\quad$ //divide by two then multiply by two after making int to preserve symmetry

// if S is null don't try to free the array because there is nothing to free

// then create new $2 \mathrm{~s}$ array for omega vs $\mathrm{K}$

if( S != NULL) free_dmatrix(S,old_rlength/2, old_krlength); //Clean memory

$\mathrm{S}=\mathrm{dmatrix}(\mathrm{rlength} / 2$, krlength); // Create a pointer to a $2 \mathrm{~d}$ array, i.e. create $2 \mathrm{~d}$

array

// This section loads information for file \#1

wave1=return_data(1);

// This section loads information for file \#2

wave2=return_data(2);

$/ /$ read in file information and if no error in the read start analysis procedures else fail with a miserable message.

if (wave1!=NULL \&\& wave2!=NULL \&\& sizeof(wave1)==sizeof(wave2)) 
\{

// Initialize arrays to zero

for $(\mathrm{i}=0 ; \mathrm{i}<$ rlength $/ 2 ; \mathrm{i}++)$

\{

ave_wave1_pow $[i]=0$;

ave_wave $2 \_$pow $[i]=0$;

for $(\bar{j}=0 ; j<$ krlength; $j++)$

\{

$\mathrm{S}[\mathrm{i}][\mathrm{j}]=0$;

\}

\}

// Find the $\mathrm{k}$ values looping through for each time series

for $(\mathrm{i}=0 ; \mathrm{i}<$ iterations; $\mathrm{i}++)$

\{

//put in values for each subset of a time series - added by E. Scime May 2008

for $(\mathrm{k}=0 ; \mathrm{k}<$ num_subsets; $\mathrm{k}++)\{$

// put in values for time series one at a time for analysis

for $(j=0 ; j<$ rlength; $j++)\{$

wave1 out $[\mathrm{j}]=$ wave $1[\mathrm{j}+$ rlength*k][i];

wave2out $[\mathrm{j}]=$ wave $2[\mathrm{j}+$ rlength*k][i];

\}

//Use Paul's analysis routine for finding $2 \mathrm{~d}$ spectral density

Find_k ( wave1out, wave2out, Theta, cross, ave_wave1_pow, ave_wave2_pow,

$\mathrm{S}$, freq);

\}

$/ /$ create frequency axis

for $(\mathrm{i}=0 ; \mathrm{i}<$ rlength $/ 2 ; \mathrm{i}++)$

\{

freq $[\mathrm{i}]=($ double $){ }^{*}$ srate/(double)rlength;

\}

// Plot ffts and cross power info

cross plot(Theta, cross, freq);

fft_plot(ave_wave1_pow, ave_wave2_pow, freq);

// set $\mathrm{x}$ axis for $\mathrm{k}$ and plot intensity and plot intensity

GetCtrlVal (main_panel, PANEL_DIST, \&dist);

GetCtrlVal (main_panel, PANEL_KLAMBDA_FLAG, \&klambda_flag);

if $\left(\mathrm{klambda} \_\right.$flag $\left.==0\right)$

\{ 
SetCtrlVal (main_panel, PANEL_XMIN, -PI/dist);

SetCtrlVal (main_panel, PANEL_XMAX, PI/dist);

\}

else

\{

SetCtrlVal (main_panel, PANEL_XMIN, -dist*PI);

SetCtrlVal (main_panel, PANEL_XMAX, dist*PI);

\}

intensity_plot( S, freq);

\}

else

\{

\}

MessagePopup ("Error", "Unable to get data from file");

old_rlength = rlength; // Store values to clear array later since rlength and krlength

old_krlength = krlength; $\quad$ // will be different when this function is called again

wave1=NULL;

wave $2=$ NULL;

return $\mathrm{S}$;

\}// end of data analysis

void Find_k (double wave1[ANALYSIS_RLENGTH], double

wave2[ANALYSIS_RLENGTH], double Theta[ANALYSIS_RLENGTH], double cross[ANALYSIS_RLENGTH], double

ave_wave1_pow[ANALYSIS_RLENGTH],double

ave_wave2_pow[ANALYSIS_RLENGTH], double ${ }^{* *}$ S, double freq[ANALYSIS_RLENGTH])

\{

//double wave1_I[ANALYSIS_RLENGTH];

//double wave2_I[ANALYSIS_RLENGTH];

//double coherence[ANALYSIS_RLENGTH];

//double coherence_length[ANALYSIS_RLENGTH];

//double ca1[ANALYSIS_RLENGTH];

//double ca2[ANALYSIS_RLENGTH];

//double phase[ANALYSIS_RLENGTH];

double wave1_pow[ANALYSIS_RLENGTH];

double wave2_pow[ANALYSIS_RLENGTH];

double real_cross_spec[ANALYSIS_RLENGTH];

double imag_cross_spec[ANALYSIS_RLENGTH];

double K[ANALYSIS_RLENGTH/2]; 
double deltak;

double wave1_mean;

double wave2_mean;

double dist;

int $\mathrm{i}$;

int $\mathrm{j}$;

int $\mathrm{n}$;

int iterations;

int rlength;

int krlength;

int kbins;

int Win_Choice; // Time series windowing option

GetCtrlVal (main_panel, PANEL_ITER, \&iterations);

GetCtrlVal (main_panel, PANEL_RLENGTH, \&rlength);

GetCtrlVal (main_panel, PANEL_DIST, \&dist);

GetCtrlVal (main panel, PANEL KBINS, \&kbins);

GetCtrlVal (main_panel, PANEL_Window_Choice, \&Win_Choice);

// get_coil_info( freq, ca1, ca2, phase);

// Find the mean for each signal

Mean(wave1, rlength, \&wave1 mean);

Mean(wave2, rlength, \&wave2_mean);

// Subtract mean from signal

for $(\mathrm{j}=0 ; \mathrm{j}<$ rlength $; \mathrm{j}++)$

\{

wave1[j] $=($ wave1[j] - wave1_mean $)$;

wave2[j] = (wave2[j] - wave2_mean);

wave1_pow[j] = wave1[j];

wave 2 pow $[\mathrm{j}]=$ wave $2[\mathrm{j}]$;

\}

switch (Win_Choice)

\{

case 0 :

Spectrum(wave1_pow, rlength);

Spectrum(wave2_pow, rlength);

break;

case 1: 
TriWin (wave1, rlength);

TriWin (wave2, rlength);

TriWin (wave1_pow, rlength);

TriWin (wave2_pow, rlength);

Spectrum(wave1_pow, rlength);

Spectrum(wave2_pow, rlength);

break;

case 2:

HanWin (wave1, rlength);

HanWin (wave2, rlength);

HanWin (wave1_pow, rlength);

HanWin (wave2_pow, rlength);

Spectrum(wave1_pow, rlength); Spectrum(wave2_pow, rlength);

case 3:

break;

HamWin (wave1, rlength);

HamWin (wave2, rlength);

HamWin (wave1_pow, rlength);

HamWin (wave2_pow, rlength);

Spectrum(wave1_pow, rlength); Spectrum(wave2_pow, rlength); break;

case 4:

BkmanWin (wave1, rlength);

BkmanWin (wave2, rlength);

BkmanWin (wave1_pow, rlength);

BkmanWin (wave2_pow, rlength);

Spectrum(wave1_pow, rlength);

Spectrum(wave2_pow, rlength);

break;

case 5:

BlkHarrisWin (wave1, rlength);

BlkHarrisWin (wave2, rlength); 
BlkHarrisWin (wave1_pow, rlength);

BlkHarrisWin (wave2_pow, rlength);

Spectrum(wave1_pow, rlength);

Spectrum(wave2_pow, rlength);

break;

/*

// Create Power Spectrum for each signal

HanWin(wave1_pow, rlength);

HanWin(wave2_pow, rlength);

Spectrum(wave1_pow, rlength);

Spectrum(wave2_pow, rlength);

// Determine the Cross Spectrum for the two coils.

$/ /$ cross is the real part (quad)

// Theta is the imaginary part

HamWin(wave 1, rlength);

HamWin(wave2, rlength);

*/

CrossSpectrum(wave1, wave2, rlength, real_cross_spec,imag_cross_spec);

$/ /$ for $(\mathrm{j}=1 ; \mathrm{j}<$ rlength $/ 2 ; \mathrm{j}++)$

for $(\mathrm{j}=0 ; \mathrm{j}<$ rlength $/ 2 ; \mathrm{j}++)$

\{

ave_wave1_pow[j] += wave1_pow[j]/iterations;

ave_wave2_pow $[\mathrm{j}]+=$ wave $2 \_$pow $[\mathrm{j}] /$ iterations;

//wave1_pow[j] *= pow $(($ rlength/2),2)*SCALE/(pow $($ ca1 $[\mathrm{j}] *$ freq[j] $/ 1 \mathrm{e} 6,2))$;

//wave2_pow $[\mathrm{j}] *=\operatorname{pow}(($ rlength/2),2)*SCALE/(pow $(\mathrm{ca} 2[\mathrm{j}] *$ freq[j]/1e6,2));

//wave1_pow $[\mathrm{j}] *=\operatorname{pow}((\operatorname{rlength} / 2), 2) /(\operatorname{pow}(\mathrm{ca} 1[\mathrm{j}], 2))$;

//wave2_pow $[\mathrm{j}] *=\operatorname{pow}((\operatorname{rlength} / 2), 2) /(\operatorname{pow}(\mathrm{ca} 2[\mathrm{j}], 2))$;

if $(\operatorname{cross}[j]==0)$

$$
\operatorname{cross}[\mathrm{j}]=1 \mathrm{e}-20 \text {; }
$$

\}

// Size of wavenumber bin

krlength $=$ kbins;

deltak $=2 *$ PI $/$ (dist * (double) krlength);

//divide by two then multiply by two after making int to preserve symetry

// Subtract the phaseshift of the coils from the measured phase shift and divide

// by the distance to determine the k-value 


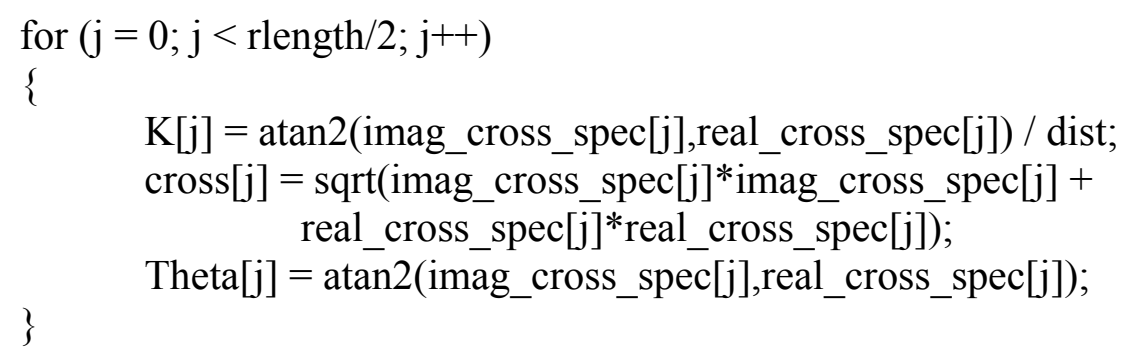

//Update S matrix with additional values each time through (a running summation) for $(j=0 ; j<\operatorname{rlength} / 2 ; j++)$

\{

for $(\mathrm{n}=0 ; \mathrm{n}<$ krlength $/ 2 ; \mathrm{n}++)$

\{

if $(\mathrm{K}[\mathrm{j}]>=0)$
if $((($ double $) n) * \operatorname{deltak}<\mathrm{K}[\mathrm{j}] \& \&(($ double $)(\mathrm{n}+1)) * \operatorname{deltak}>\mathrm{K}[\mathrm{j}])$

$\mathrm{S}[\mathrm{j}][((\mathrm{krlength} / 2)+\mathrm{n})]+=\left(.5 *\left(\right.\right.$ wave $2 \_$pow $[\mathrm{j}]+$ wave1_pow $\left.\left.[\mathrm{j}]\right)\right) ; / /$ spectral density else \}

$\mathrm{S}[\mathrm{j}][((\mathrm{krlength} / 2)+\mathrm{n})]+=0$

\}

else

if $((($ double $)(-n-1)) * \operatorname{deltak}<K[j] \& \&(($ double $)(-n)) * \operatorname{deltak}>K[j])$

\{

$\mathrm{S}[\mathrm{j}][((\mathrm{krlength} / 2)-\mathrm{n}-1)]+=(.5 *$ (wave2_pow $[\mathrm{j}]+$ wave1_pow[j] $)) ; / /$ spectral density

\}

else

$\mathrm{S}[\mathrm{j}][((\mathrm{krlength} / 2)-\mathrm{n}-1)]+=0$;

\}

\}

\}

\} 
Vitae

\section{Robert Aaron Hardin}

\section{Education}

Ph.D. Plasma Physics October 2008

West Virginia University, Morgantown, WV 26506

M.S. Plasma Physics December 2005

West Virginia University, Morgantown, WV 26506

B.S. Engineering-Physics May 2001

West Virginia Wesleyan College, Buckhannon, WV 26201

\section{Professional Experience}

May 2002 - Present

Graduate Research Assistant Experience:

- $300 \mathrm{GHz}$ quasi-optical collective Thomson scattering diagnostic

o Investigation of the lower hybrid wave in a helicon plasma source

o Development of a homodyne detection scheme employing a single Gunn oscillator source and Schottky diode detector

o Design and manufacture of Gaussian beam quasi-optical elements (Zemax Gaussian beam propagation program for design)

- Helicon plasma source (design, construction, and operation)

- Instrumentation, data acquisition, and data analysis programming in $\mathrm{C}$, LabWindows, and Matlab

- $\quad$ Laser Induced Fluorescence (LIF)

o Ring dye laser and tunable diode laser systems

o Design and construction of conventional optical components

o Measurement of 3D flows in argon plasmas

- Emission and absorption spectroscopy

- Electrostatic probes

o Langmuir, double, and retarding field energy analyzer

- Electronic design and construction

o Signal amplifiers, matching networks, RF noise reduction

- General laboratory equipment design and construction

o Computer Aided Design (CAD) in AutoCad LT and Graphite

o Machining, soldering, and plumbing

o Vacuum technology

- High energy density plasmas 
o Short-pulse single-hot-spot experiments to investigate stimulated Raman scattering (Los Alamos National Laboratory)

- Advanced computational techniques

o Training in use of LSP PIC code (Princeton Plasma Physics Laboratory)

- Mentoring of undergraduate research students

September 2001 - May 2002

Graduate Teaching Assistant Experience:

- Laboratory instructor for introductory physics (algebra based)

- Introductory physics exam grading

- Introductory physics lecturer (substituted for 3 lectures - algebra based)

\section{Graduate Courses within Research Concentration}

Principles of Plasma Physics

Advanced Kinetic Theory of Plasma

Advanced Magnetohydrodynamic Theory of Plasma

Computer Simulation of Plasma

Nonlinear Dynamics

\section{Additional Professional Education}

UCLA/University of Maryland Winter School (2007) - Plasma Turbulence and Transport

UCLA/University of Maryland Winter School (2006) - Magnetic Reconnection

\section{Professional Memberships}

American Physical Society (APS)

Division of Plasma Physics (DPP)

Sigma Pi Sigma - National Physics Honor Society

\section{Invited Talks and Colloquia}

Development of a $300 \mathrm{GHz}$ Collective Scattering Diagnostic on the WVU Helicon

Plasma Source, Oak Ridge National Lab, April, 2008.

Plasma Physics Research at WVU and Los Alamos National Lab, West Virginia

Wesleyan College, April, 2007.

Plasma Flows: Friend or Foe of Fusion Plasmas, West Virginia Wesleyan College, March, 2006. 


\section{$\underline{\text { Publications }}$}

\section{Refereed Journals, First Author}

R. Hardin, E.E. Scime, J. Heard, "A $300 \mathrm{GHz}$ Collective Scattering Diagnostic for Low Temperature Plasmas,” Rev. Sci. Instrum. 79, 10E725 (2008).

R. Hardin, X. Sun, E.E. Scime, "Three-dimensional laser-induced fluorescence measurements in a helicon plasma," Rev. Sci. Instrum. 75, 4103 (2004).

\section{Refereed Journals, Co-author}

W.S. Przybysz, J. Ellis, A. Hansen, R. A. Hardin, and E.E. Scime, "A magneto-optic probe for magnetic fluctuation measurements in a plasma," Submitted to Rev. Sci. Instrum. (2008).

Z. Harvey, S. Chakraborty Thakur, A. Hansen, R. Hardin, W.S. Przybysz, E.E. Scime, "Comparison of Gridded Energy Analyzer and Laser Induced Fluorescence Measurements of a Two-Component Ion Distribution," Rev. Sci. Instrum. 79, 10F314 (2008).

J. L. Kline, D. S. Montgomery, L. Yin, R. A. Hardin, K. A. Flippo, T. Shimada, R. P. Johnson, H. A. Rose, and B. J. Albright, "Investigation of laser plasma instabilities using picoseconds laser pulses," J. Phys.: Conf. Ser. 112, 022042 (2008).

E. Scime, R. Hardin, C. Biloiu, A. M. Keesee, X. Sun, "Flow, flow shear, and related profiles in helicon plasmas," Phys. Plasmas. 14, 043505 (2007).

X. Sun, C. Biloiu, R. Hardin, E.E. Scime, "Parallel velocity and temperature of argon ions in an expanding, helicon source driven plasma," Plasma Sources Sci. Technol. 13, 359 (2004).

J.L. Kline, M.M. Balkey, P.A. Keiter, E.E. Scime, A.M. Keesee, X. Sun, R. Hardin, C. Compton, R.F. Boivin, M.W. Zintl, "Ion dynamics in helicon sources," Phys. Plasmas 10, 2127 (2003).

\section{Conference Papers Presented (First Author Only)}

R. Hardin, Earl Scime, Alex Hansen, "A 300 GHz Collective Scattering Diagnostic for Low Temperature Plasmas,” HTPD (2008).

R. Hardin, Earl Scime, Alex Hansen, "Slow wave measurement using the WVU 300 GHz collective scattering diagnostic," APS Div. of Plasma Physics (2007).

R. Hardin, Earl Scime, Mike Spencer, "Progress towards measurement of the slow wave in the WVU Helicon Plasma Source," APS Div. of Plasma Physics (2006). 
R. Hardin, John Heard, Earl Scime, Mike Spencer, Ryan Murphy, Zane Harvey, "A $300 \mathrm{GHz}$ Collective Scattering System for Low Temperature Plasmas," APS Div. of Plasma Physics (2005).

R. Hardin, John Heard, Earl Scime, Mike Spencer, Ryan Murphy, Zane Harvey, "A $300 \mathrm{GHz}$ Collective Scattering System for Low Temperature Plasmas," IRMMW$\mathrm{THz}$ Conference (2005).

R. Hardin, Earl Scime, Mike Spencer, John Heard, "Coherent Scattering Diagnostic for Short Wavelength Fluctuations," IPELS (2005).

R. Hardin, C. Biloiu, C. Compton, A.M. Keesee, E.E. Scime, J. Heard, D. Ventura, "Evolution of the Ion Velocity Distribution Function in a Pulsed Helicon Source," APS Div. of Plasma Physics (2004).

R. Hardin, E.E. Scime, C. Biloiu, J. Heard, "Collective Scattering Technique for SubMillimeter Wavelength Fluctuation,” ICOPS (2004).

R. Hardin, X. Sun, E.E. Scime, "3D LIF measurements in a helicon plasma," HTPD (2004).

R. Hardin, C. Biloiu, C. Compton, A.M. Keesee, E.E. Scime, X. Sun, "3D Measurements of the Ion Flow Velocity in a Rotating Helicon Plasma," APS Div. of Plasma Physics (2003).

R. Hardin, C. Compton, A.M. Keesee, X. Sun, E.E. Scime, J.L. Kline, R.F. Boivin, "Rotational Flow Measurements in a Helicon Plasma Source," APS Div. of Plasma Physics (2002). 